\title{
A Deviatoric Softening Model to Simulate Compressibility Properties of Soft Clays
}

\author{
by \\ Cong Shi \\ B.Sc., Southwest Jiaotong University, China, 2012 \\ M.Eng., Southwest Jiaotong University, China, 2015 \\ A dissertation \\ presented to Ryerson University \\ in partial fulfillment of the \\ requirements for the degree of \\ Doctor of Philosophy \\ in the program of \\ Civil Engineering
}

Toronto, Ontario, Canada, 2019

(C) Cong Shi, 2019 


\section{AUTHOR'S DECLARATION}

I hereby declare that I am the sole author of this dissertation. This is a true copy of the dissertation, including any required final revisions, as accepted by my examiners.

I authorize Ryerson University to lend this dissertation to other institutions or individuals for the purpose of scholarly research.

I further authorize Ryerson University to reproduce this dissertation by photocopying or by other means, in total or in part, at the request of other institutions or individuals for the purpose of scholarly research.

I understand that my dissertation may be made electronically available to the public. 


\title{
A Deviatoric Softening Model to Simulate Compressibility Properties of Soft Clays
}

\author{
Cong Shi, Doctor of Philosophy, 2019 \\ Department of Civil Engineering \\ Ryerson University, Toronto, Canada
}

\begin{abstract}
Soft clays are often associated with high compressibility due to their high void ratio, low shear strength, and creep behavior. Structures built on top of it can experience excessive settlement issues over a long period of time. The prediction of these settlements has attracted attentions from many researchers for over a century, but accurately predicting them still remains a difficult issue due to complex properties of soft clays, including plasticity, viscosity, anisotropy, soil structure and so forth. Therefore, studying the compressibility of soft clay is of significant importance. This dissertation aims to investigate the influence of plastic deviatoric strains on the compressibility of soft clays.
\end{abstract}

First of all, the dissertation reviews a number of published incremental anisotropic consolidation tests on Finnish clays. The results demonstrate the dependence of soil compressibility on stress ratios. Based on the result, a modified yield surface size deviatoric softening law has been introduced. This softening law describes yield surface softening to be related to plastic deviatoric strain increments.

Secondly, a new model named MEVP-DS, has been incorporated into the framework of Yin's elaso-viscoplastic model to consider deviatoric softening, destructuration, and yield surface anisotropy of soft clay.

Furthermore, the verification of MEVP-DS has been done through three phases. Phase one is the simulation of published incremental anisotropic consolidation tests on intact Finnish clay samples. The model results demonstrate MEVP-DS successfully captures the soil compressibility in response to different stress ratios. Phase two is the application of MEVP-DS in modeling 1-D 
consolidation tests on sensitive Champlain Sea clay. Model results highlight that using MEVP-DS is beneficial for predicting the compressibility and excess pore pressure response of the clay subject to constant rate of strain loading. Phase three is the application of MEVP-DS in simulating a real embankment dam on Champlain Sea clay. MEVP-DS not only simulates 40-year settlement measurements of the dam reasonably well, but also improves the prediction of lateral spreading of the dam.

In summary, the MEVP-DS model proposed in this dissertation has shown to improve the simulation of soil compressibility of soft clays subject to 1-D, anisotropic and more complicated loading conditions. 


\section{ACKNOWLEDGEMENTS}

First of all, I would like to extend my sincere gratitude to Dr. Jinyuan Liu. He is my supervisor and a great guide who helped me tackle technical issues, and a great mentor who provided indispensable life experience to his students. Without him, I wouldn't have had this wonderful opportunity to do research and survive in Toronto, and to spend my memorable four years at Ryerson University. Again, I am tremendously thankful to him.

Secondly, I would like to thank my co-supervisor, Dr. Tareq Salloum from Ontario Power Generation, for providing the opportunity and financial assistance for the Waba dam project. This thesis couldn't have been done without the precious Laval samples OPG shared with us. Dr. Tareq Salloum is also a humble engineer, a patient teacher, and an empathetic listener. It has been an honor to be supervised by him.

Next, I would love to mention Dr. Jianhua Yin, who shared his FORTRAN codes of MEVP model with us. I'm extremely grateful for this generosity, as my work would not have been possible without his model. I also want to mention Dr. Laifa Cao for his valuable inputs from the very beginning of my Ph.D. study.

Furthermore, I want to mention China Scholarship Council for the financial support, Mitacs Accelerate for offering the internship opportunity, Hatch for inviting me over for the technical visit.

Last but not the least, I want to thank Dr. Peiyuan Lin for his suggestions and inputs, Mr. Zhengliang Jiang for sharing his visions, Mr. Andries Kirstein and Mr. Ali Ahmad for helping with the tests, and Mr. Shuihan Li for his help and advice. I'm also greatly thankful to my mother and my family, whose support has been always unwavering. 


\section{TABLE OF CONTENTS}

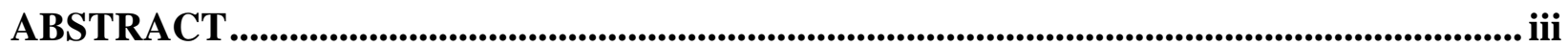

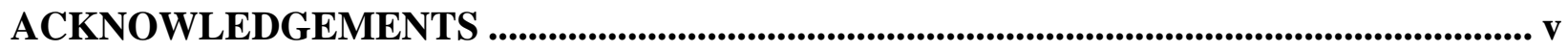

TABLE OF CONTENTS ................................................................................................. vi

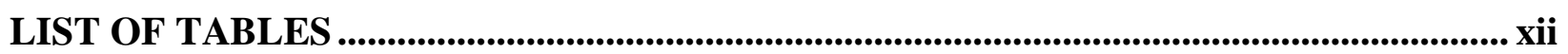

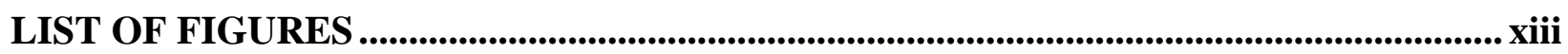

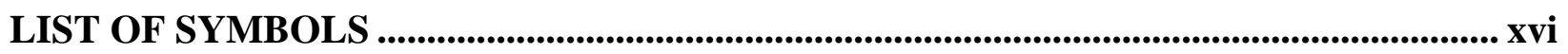

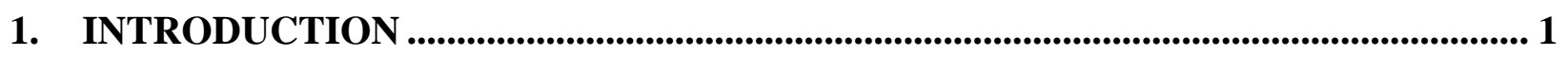

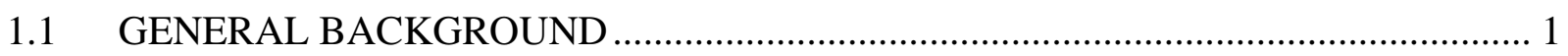

1.1.1 Clays and their compressibility properties......................................................... 1

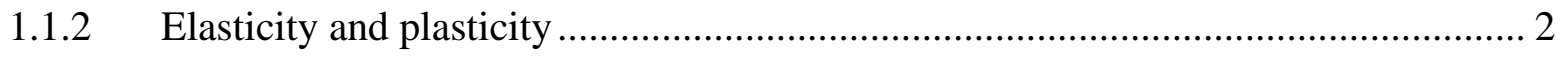

1.1.3 Strain hardening and strain softening phenomena …............................................ 3

1.1.4 Soil compressibility and plastic strains .............................................................. 3

1.1.5 Settlement issues of Waba dam built on Champlain Sea clay .................................. 4

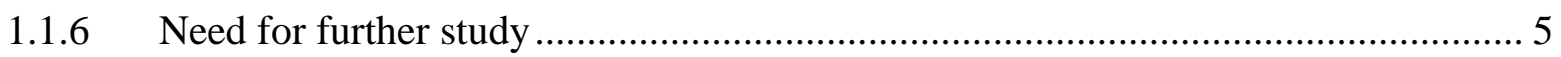

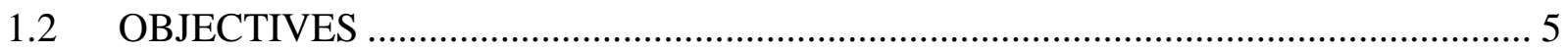

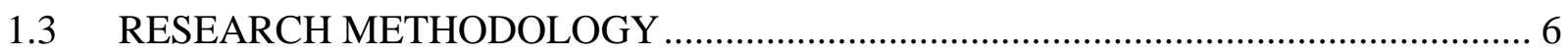

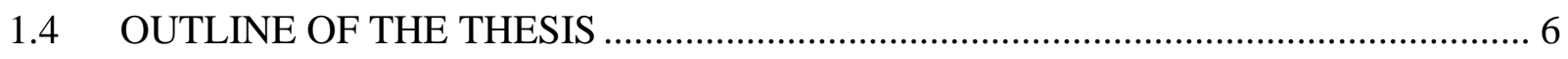

2. LITERATURE REVIEW: NUMERICAL MODELING OF SOFT CLAY

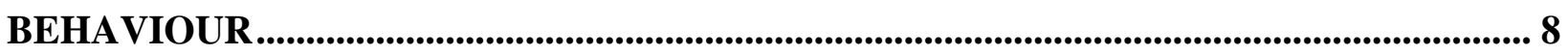

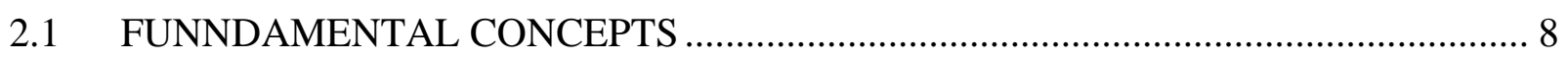

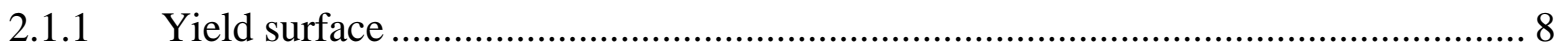




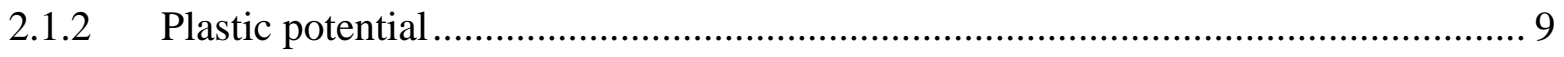

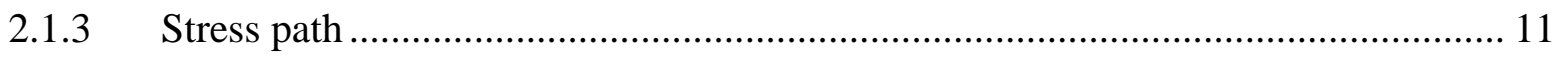

2.2 YIELD SURFACE AND FAILURE SURFACE OF SOIL ……………………........ 11

2.3 YIELD SURFACE HARDENING MODELS ……………………........................... 13

2.3.1 Yield surface volumetric hardening models for sand and clay .............................. 13

2.3.2 Yield surface biased hardening models for sand and clay .................................... 14

2.4 DISTORTIONAL HARDENING AND SOFTENING MODELS .............................. 15

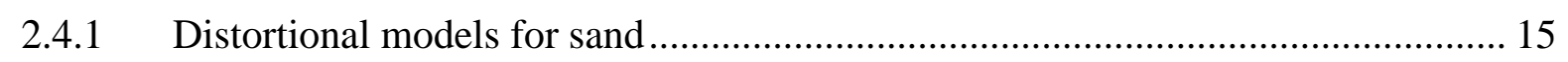

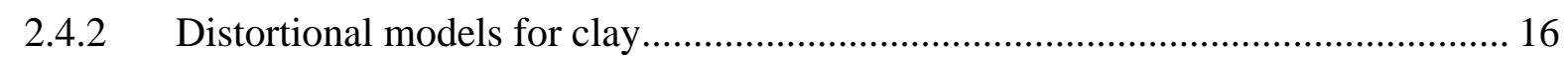

2.5 YIELD SURFACE DESTRUCTURATION THEORIES ……………………........... 17

2.5.1 Koskinen's destructuration model ...................................................................... 17

2.5.2 Baudet and Stallebrass's destructuration model ................................................... 18

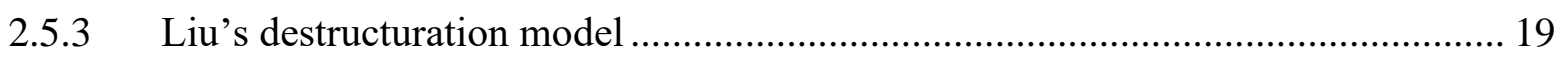

2.6 YIELD SURFACE ANISOTROPY THEORIES ……………………………......... 20

2.6.1 Rotational anisotropy theories ........................................................................ 20

2.6.2 Multi-laminate anisotropy theories .................................................................... 22

2.7 COMPRESSIBILITY OF FINNIFHS SOFT CLAYS SUBJECT TO IAC TESTS...... 23

2.7.1 Introduction to incremental anisotropic consolidation tests .................................. 23

2.7.2 Engineering background of Finnish marine clays .............................................. 24

2.7.3 Experimental results of IAC tests on remolded Murro clay …………………........ 25

2.7.4 Experimental results of IAC tests on remolded Otaniemi clay.............................. 27

2.7.5 Experimental results of IAC tests on Otaniemi clay …………………………...... 28

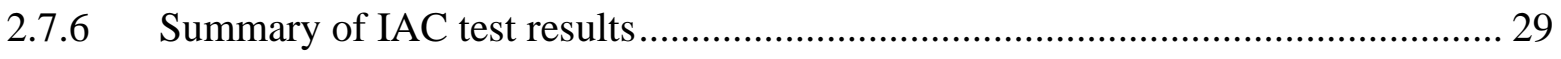

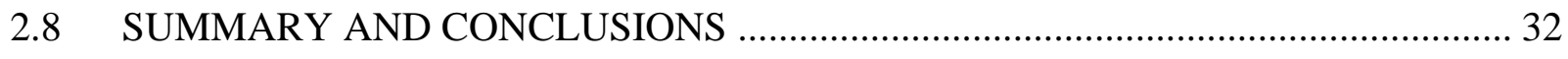

3. DEVELOPMENT OF A DEVIATORIC SOFTENING FOR SOFT CLAYS ............... 33 


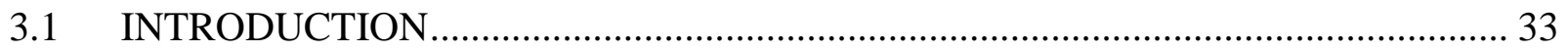

3.2 A MODIFIED DEVIATORIC SOFTENING LAW FOR SOFT CLAYS …………...... 33

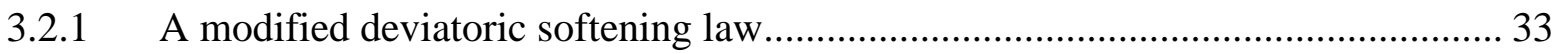

3.2.2 Family of compression lines of the new softening law........................................ 35

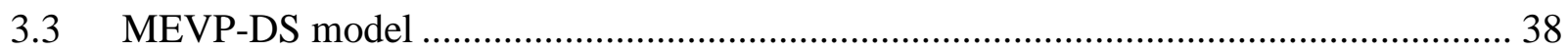

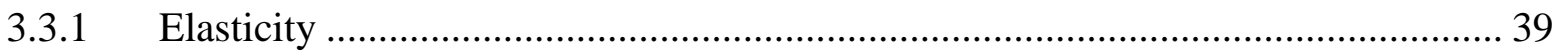

3.3.2 Evolutional anisotropy of yield surface …………….......................................... 39

3.3.3 Visco-plasticity of time-dependence behavior........................................................ 41

3.3.4 Destructuration of structured yield surface ...................................................... 43

3.3.5 Implementation of MEVP-DS in Plaxis V8.2 .................................................... 43

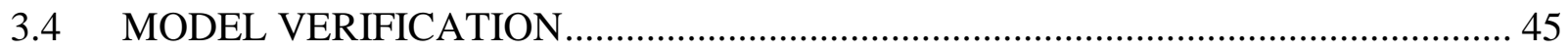

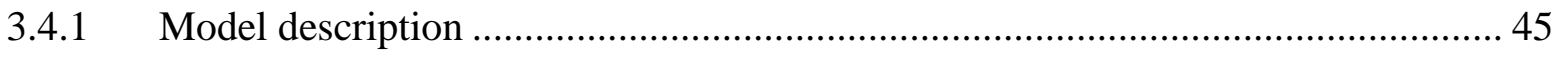

3.4.2 Parameters input of MEVP-DS model................................................................. 46

3.4.3 Simulation of IAC tests on Finnish clays …………………………………........ 49

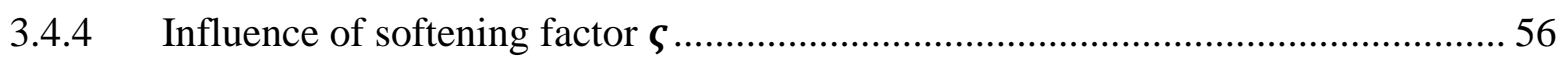

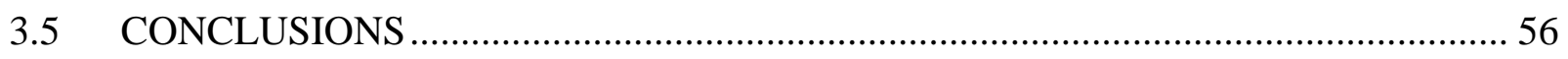

\section{MODEL VERIFICATION: MODELING 1-D COMPRESSION OF CHAMPLAIN}

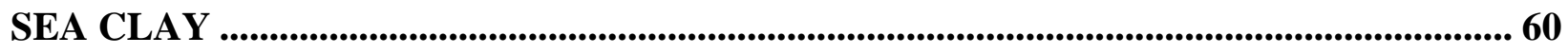

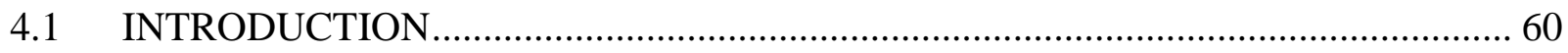

4.2 THE FORMATION AND THE PROPERTIES OF CHAMPLAIN SEA CLAY .......... 60

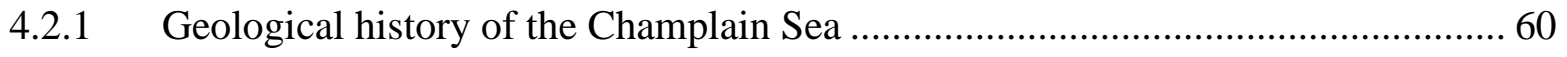

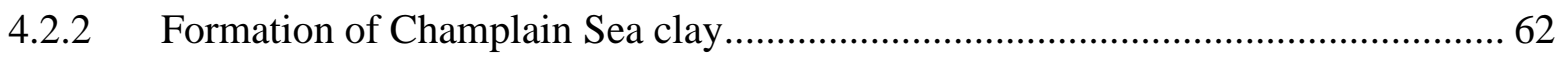

4.2.3 Engineering properties of Champlain Sea clay ...................................................... 64

4.3 BACKGROUND OF CRS CONSOLIDATION TEST ................................................ 67 


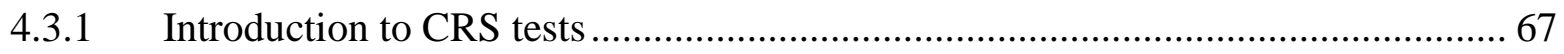

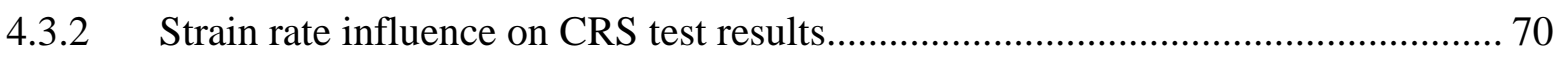

4.3.3 CRS testing system at Ryerson University and sample preparation....................... 70

4.4 NUMERICAL SIMULATION OF CRS TESTS ON CHAMPLAIN SEA CLAY ....... 73

4.4.1 Overview of CRS test results on Champlain Sea clay ........................................... 73

4.4.2 Model description for CRS test simulation......................................................... 75

4.4.3 Parameters input for CRS test simulation ........................................................... 76

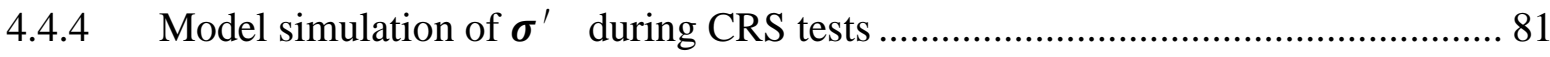

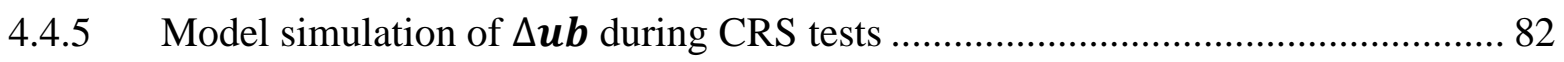

4.5 SENSITIVITY ANALYSIS OF INPUT PARAMETERS OF MEVP-DS MODEL .... 89

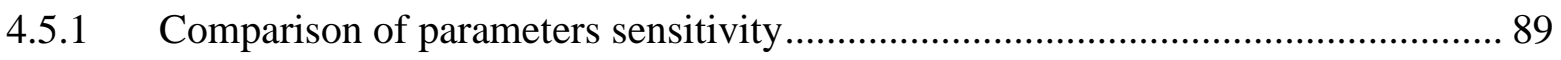

4.5.2 Strain rate effect on model predictions ……………………................................ 94

4.6 SIMULATION OF OEDOMETER TESTS …………………………………......... 95

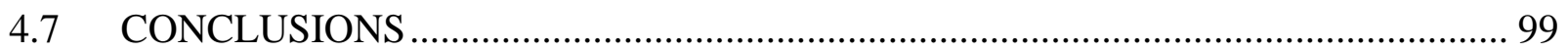

5. MODEL VERIFICATION: MODELING SETTLEMENT OF WABA DAM ON SENSITIVE CHAMPLAIN SEA CLAY FOUNDATION_.................................................... 100

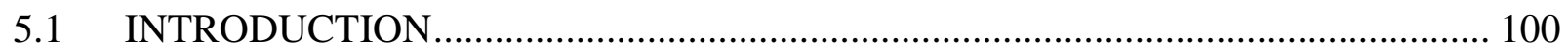

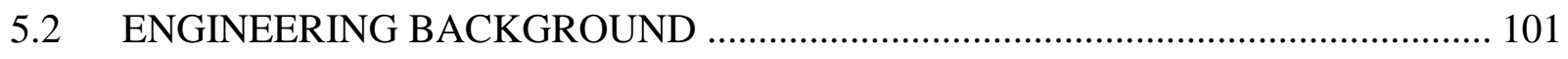

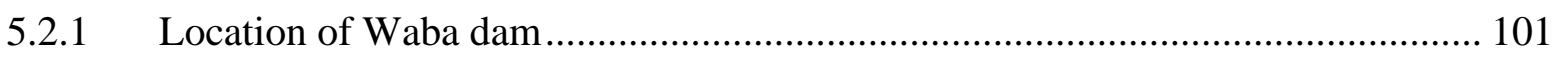

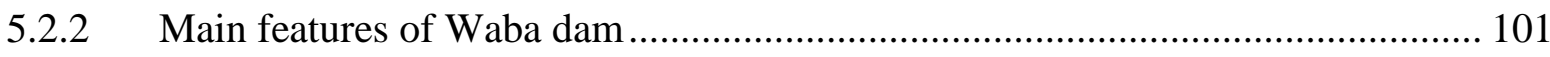

5.2.3 Construction schedule of Waba dam .............................................................. 103

5.2.4 Instrumentations and measurements ............................................................. 103

5.3 Geotechnical characteristics of Champlain Sea clay .................................................... 105

5.3.1 Physical properties of the soil samples ........................................................... 105 
5.3.2 Moisture contents and natural densities ................................................... 106

5.3.3 Undrained shear strength and sensitivity ................................................. 108

5.3.4 Critical state soil properties ..................................................................... 109

5.3.5 Preconsolidation Pressure and OCR .......................................................... 111

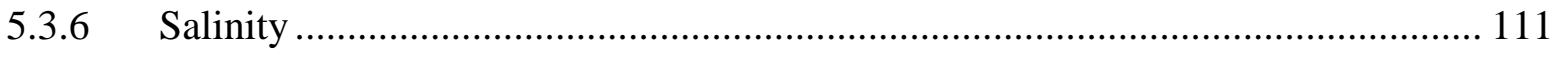

5.4 GEOMETRY AND MODEL DETAILS OF WABA DAM ................................. 112

5.5 MODEL PARAMETERS FOR FOUNDATION CLAY ..................................... 114

5.6 FOUNDATION AND CREST SETTLEMENT ............................................... 117

5.6.1 Construction settlement of dam foundation .............................................. 117

5.6.2 Long-term settlement of dam crest ........................................................ 118

5.6.3 Simulated foundation settlement profile in selected years ............................... 120

5.7 EXCESS PORE PRESSURE IN THE FOUNDATION ........................................ 122

5.7.1 Excess pore pressure immediately after construction ..................................... 122

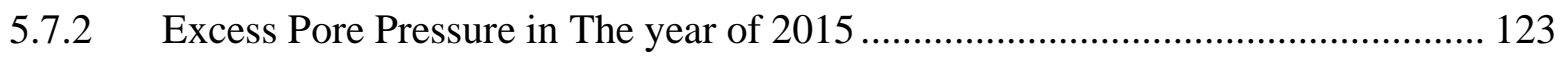

5.7.3 Simulated $\Delta \boldsymbol{u}$ dissipation of centreline sections ....................................... 124

5.8 LATERAL SPREADING IN THE FOUNDATION .......................................... 128

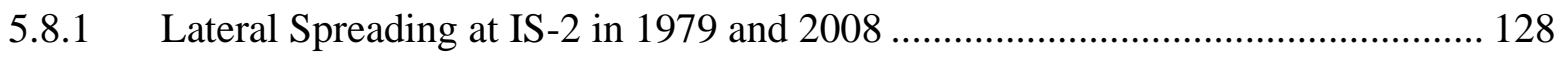

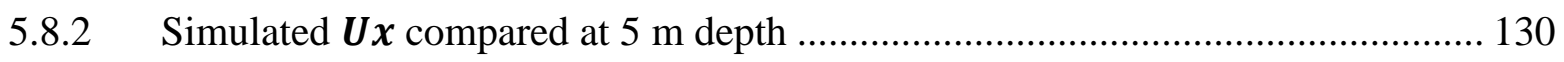

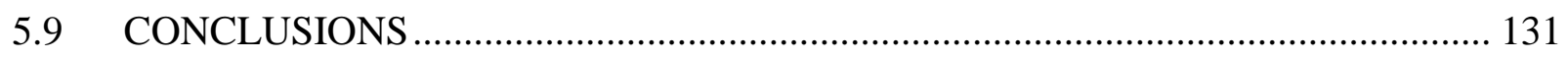

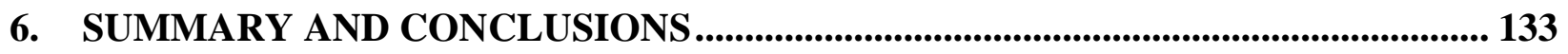

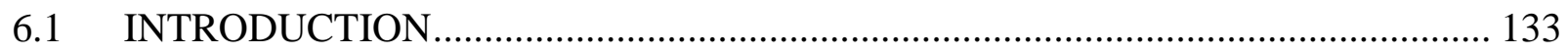

6.2 MAIN CONCLUSIONS ............................................................................... 134

6.3 SIGNIFICANCE OF THIS RESEARCH .................................................... 135

6.4 FUTURE RESEARCH .............................................................................. 135 
APPENDIX A - CODE OF SUBROUTINE (EVP.for) .............................................................. 137

APPENDIX B - CODE OF SUBROUTINE (MY_CLAY.for) ........................................... 149

REFERENCES 


\section{LIST OF TABLES}

Table 3.1 Main tasks to be defined in a Plaxis subroutine for user-defined model ................... 44

Table 3.2 Other subroutines that need to be called by the main subroutine ............................. 44

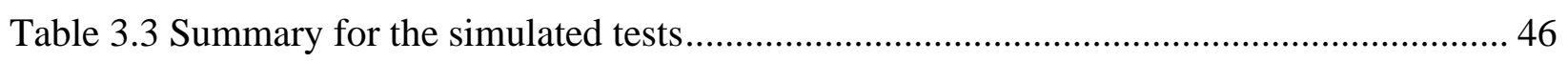

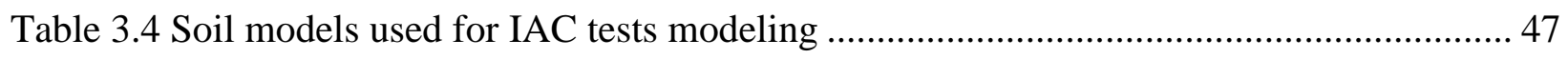

Table 3.5 Values for the initial state parameters used in the test modeling (collected from

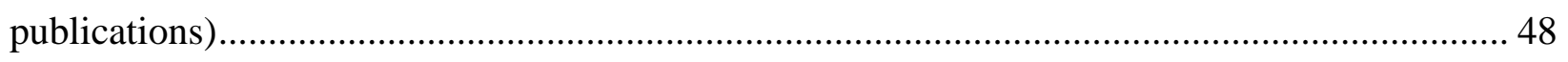

Table 3.6 Compressibility parameters for MEVP-DS ................................................... 48

Table 4.1 Soil models used for CRS tests modeling.......................................................... 76

Table 4.2 Clarification of different $\lambda$ used for modeling ................................................... 77

Table 4.3 Initial values of the parameters for MEVP-DS model used in the simulation ............ 80

Table 4.4 Initial values of the parameters for MEVP, S-CLAY1S, S-CLAY1, and SCC used in the

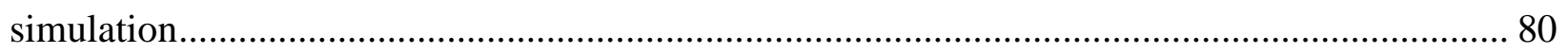

Table 5.1 Details of all six cases of parameter inputs for the foundation clay ........................ 114

Table 5.2 M-C model parameters used for weighting berm, dam, sand and till layer............... 115

Table 5.3 Model parameters used for MEVP, EVP+S-CLAY1, EVP, and S-CLAY1S ........... 115

Table 5.4 MEVP-DS model parameters used for clay foundation and trench backfill ............. 116 


\section{LIST OF FIGURES}

Figure 1.1 Work hardening and softening of soil during unconfined compression tests ............. 2 Figure 1.2 Same soil compressibility resulted from various stress ratios modelled by MCC model

Figure 2.1 Modified Cam-Clay yield surface 8

Figure 2.2 Plastic potential and yield surface of soil ......................................................... 10

Figure 2.3 Stress paths of soil subject to conventional CIU and CID loading ......................... 11

Figure 2.4 Yield surface (cap surface) and failure surface of soil ........................................ 12

Figure 2.5 Volumetric hardening law of MCC model ...................................................... 13

Figure 2.6 Koskinen's definition of soil structure in 3-D case (Koskinen et al. 2002) ............... 18

Figure 2.7 3-D case of Liu's destructuration model (Liu and Carter 2000b) ........................... 20

Figure 2.8 The inclined yield surface assumed in rotational anisotropy models (Wheeler et al. 2003)

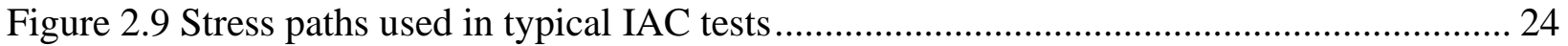

Figure 2.10 Location of Murro and Otaniemi, Finland ..................................................... 25

Figure 2.11 Results of IAC tests on remolded Murro clay (Karstunen and Koskinen 2008) ....... 26

Figure 2.12 Results of IAC tests on remolded Otaniemi clays (Karstunen and Koskinen 2008). 27

Figure 2.13 Results of IAC tests on undisturbed Otaniemi clay (Wheeler et al. 2003).............. 28

Figure 2.14 Results of IAC tests on undisturbed Otaniemi clay (Wiltafsky et al. 2002) ............ 29

Figure 2.15 Summary of dependence of compression indices on stress ratios......................... 30

Figure 2.16 Summary of dependence of compression indices on normalized stress ratios.......... 31

Figure 3.1 Critical state line and intersection of Modified Cam-Clay yield surface with different

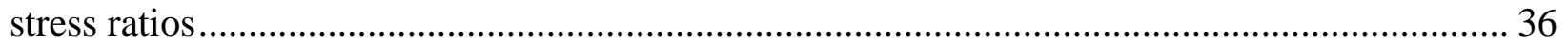

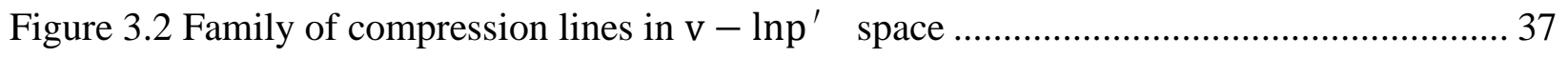

Figure 3.3 Inclined yield surfaces of MEVP-DS model (Yin and Karstunen 2008) .................. 40

Figure 3.4 Axisymmetric sample geometry for test IAC modeling ...................................... 45

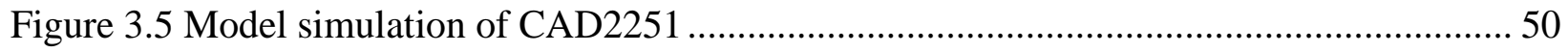

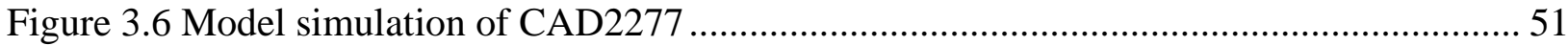

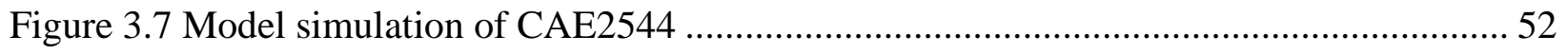

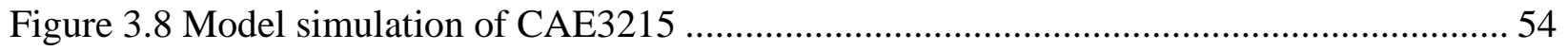




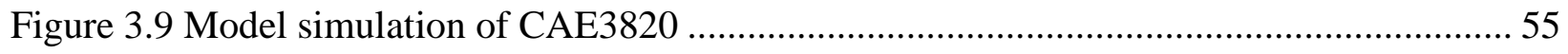

Figure 3.10 Sensitivity of $\varsigma$ to the simulation of CAD2261 ...................................................... 58

Figure 3.11 Sensitivity of $\varsigma$ to the simulation of CAD3524 ………………............................. 59

Figure 4.1 Regional map showing the extent of the Champlain Sea in the St. Lawrence River

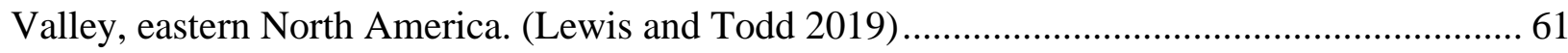

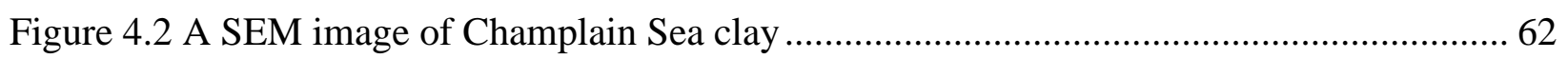

Figure 4.3 Electrical double layer theory and electrokinetics (Penner 1965)................................ 63

Figure 4.4 Chemical composition of Champlain Sea clay (Liu et al. 2017b) ............................... 64

Figure 4.5 The loss of shear strength in Champlain Sea clay due to remolding............................ 65

Figure 4.6 MIT general-purpose CRS apparatus (Wissa 1971) ..................................................... 69

Figure 4.7 CRS test system employed at Ryerson University ................................................... 71

Figure 4.8 Connection of consolidation cell used at Ryerson University (Trautwein, 2001) ...... 72

Figure 4.9 Trimming of Champlain Sea clay samples for CRS tests .......................................... 72

Figure $4.10 \varepsilon v \sim \log \left(\sigma^{\prime} / \sigma c^{\prime}\right)$ of the tested Champlain Sea Clay …………………………....... 74

Figure $4.11 \varepsilon v \sim k$ dependence of the tested Champlain Sea clay ………………...................... 74

Figure 4.12 Plaxis axisymmetric geometry for CRS test modeling............................................. 76

Figure 4.13 Estimation of $\kappa$ and $\lambda_{\mathrm{i}}$ from CRS test on Champlain Sea Clay ................................ 78

Figure 4.14 Simulation of CRS tests of Champlain Sea clay …………….............................. 82

Figure 4.15 Excess pore pressure response of Champlain Sea clays during CRS loading........... 84

Figure 4.16 Excess pore pressure response of CRS test on $4.0 \mathrm{~m}$ depth sample.......................... 85

Figure 4.17 Excess pore pressure response of CRS test on $30.4 \mathrm{~m}$ depth sample........................ 86

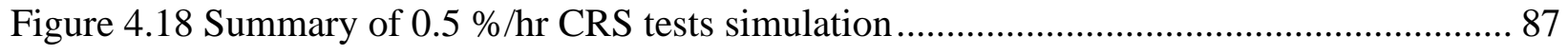

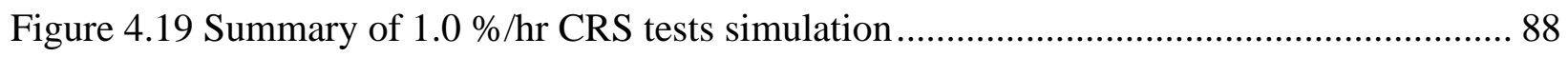

Figure 4.20 Sensitivity of MEVP-DS model parameters in simulating volumetric strain ........... 91

Figure 4.21 Sensitivity of MEVP-DS model parameters in simulating excess pore pressure...... 91

Figure 4.22 Sensitivity of MEVP-DS model parameters in simulating hydraulic gradient ......... 92

Figure 4.23 Summary of parameter sensitivities for model predictions ....................................... 93

Figure 4.24 Strain rate effects on model predictions ............................................................... 95

Figure 4.25 Simulation of oedometer tests on the foundation soil ............................................. 97

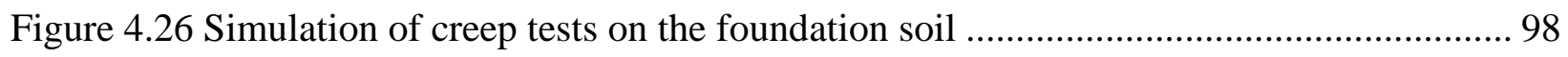

Figure 5.1 Satellite view of the site and its surrounding (Google Map, June $3^{\text {rd }}$, 2019) ............ 101 
Figure 5.2 Instrumentation of Waba dam (Cragg 1988) .................................................. 102

Figure 5.3 Typical cross section of Waba dam (Hydro 1976)............................................ 102

Figure 5.4 Dam construction sequences used in the numerical analysis ............................... 103

Figure 5.5 Instrumentation plan in Area 1 region............................................................ 104

Figure 5.6 Examples of Champlain Sea clay taken from Waba dam foundation ..................... 106

Figure 5.7 Water content, Atterberg limits and void ratio profiles of the clay foundation ........ 107

Figure 5.8 Plasticity chart of foundation clays of Waba dam .............................................. 107

Figure 5.9 Bulk density of Waba dam foundation .......................................................... 108

Figure 5.10 Undrained shear strength profile of Waba dam foundation ................................ 109

Figure 5.11 Summary of critical state points of Waba dam foundation (Liu et al. 2017) ......... 110

Figure 5.12 Pre-consolidation pressure profile of Waba dam foundation .............................. 111

Figure 5.13 Salinity profiles of Waba dam foundation...................................................... 112

Figure 5.14 FEM geometry of Area 1 cross section ...................................................... 113

Figure 5.15 Settlement at different elevations due to dam constructions .............................. 117

Figure 5.16 Comparison between the measured and predicted crest settlement ..................... 119

Figure 5.17 Comparison between the settlements predicted by different models .................... 120

Figure 5.18 Simulated foundation settlement profiles at different depths ............................ 121

Figure 5.19 Simulated centreline settlement with time at different depths ........................... 122

Figure 5.20 Piezometer instrumentation in Area 1 region ................................................ 123

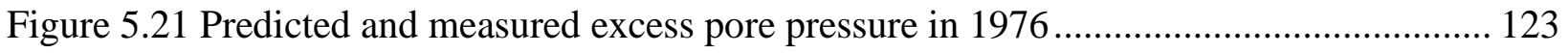

Figure 5.22 Excess pore pressure distribution with depth along the dam centreline in $2015 \ldots . .124$

Figure 5.23 Simulated $\Delta u$ dissipation at foundation centerline .......................................... 126

Figure 5.24 Simulated $\Delta \mathrm{u}$ dissipation at foundation centreline plotted in log scale................. 127

Figure 5.25 Lateral spreading of SI-2 compared with numerical prediction ........................... 129

Figure 5.26 Normalized lateral spreading of SI-2 compared with numerical prediction .......... 129

Figure 5.27 umax/s ratio as a function of time ................................................................. 130

Figure 5.28 Simulated development of Ux at $5 \mathrm{~m}$ depth with time ................................... 131 


\section{LIST OF SYMBOLS}

$\begin{aligned} \mathrm{CAD} & =\text { Anisotropically consolidated drained shear test } \\ \mathrm{CAE} & =\text { Anisotropically consolidated drained extension test } \\ \mathrm{CID} & =\text { Isotropically consolidated drained shear test } \\ \mathrm{CIU} & =\text { Isotropically consolidated undrained shear test } \\ \mathrm{CRS} & =\text { Constant rate of strain } \\ \mathrm{CSL} & =\text { End of primary consolidation } \\ \mathrm{EOP} & =\text { Elasto-viscoplastic } \\ \varepsilon_{d} & =\text { Dolute line } \\ \varepsilon_{v} & =\text { Finite element method } \\ \varepsilon_{e} & =\text { Incremental anisotropic consolidation } \\ \mathrm{FEM} & =\end{aligned}$




$$
\begin{aligned}
d \varepsilon_{d} & =\text { Deviatoric strain increment } \\
d \varepsilon_{v}^{e} & =\text { Elastic volumetric strain increment } \\
d \varepsilon_{d}^{e} & =\text { Elastic deviatoric strain increment } \\
d \varepsilon_{v}^{p} & =\text { Plastic volumetric strain increment } \\
d \varepsilon_{d}^{p} & =\text { Plastic deviatoric strain increment } \\
d \varepsilon_{v}^{v p} & =\text { Visco-plastic volumetric strain increment } \\
d \varepsilon_{d}^{v p} & =\text { Visco-plastic deviator strain increment } \\
p^{\prime} & =\text { Mean effective stress } \\
q & =\text { Deviatoric stress } \\
\eta & =\text { Stress ratio, } \eta=q / p^{\prime} \\
\eta_{0} & =\text { Initial stress ratio } \\
C_{m}^{\prime} & =\text { Size of yield surface } \\
C_{c} & =\text { Size of yield surface increment } \\
p_{m}^{\prime} & =\text { Size of intrinsic yield surface } \\
p_{m}^{\prime} & =\text { Slope of the } e \sim \log p^{\prime} \text { curve in the overconsolidated stress range } \\
p_{m} & =\text { log } p^{\prime} \text { curve in the normally consolidated stress range } \\
& =
\end{aligned}
$$




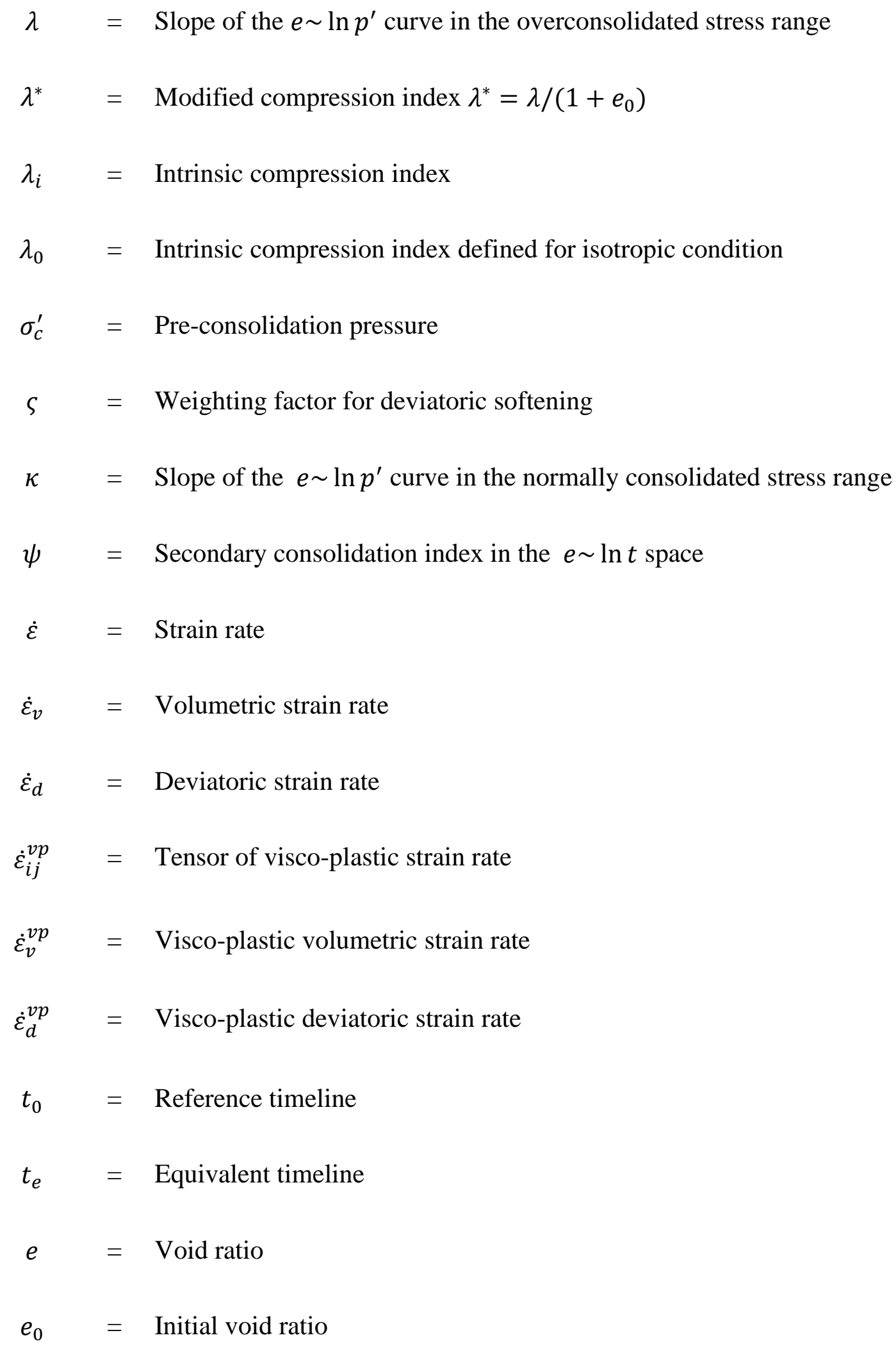




$$
\begin{aligned}
v & =\text { Specific volume, } v=1+e \\
\Gamma & =\text { Initial specific volume of CSL at unit mean effective stress }(1 \mathrm{kPa}) \\
N & =\text { Initial specific volume of NCL at unit mean effective stress }(1 \mathrm{kPa}) \\
\varphi^{\prime} & =\text { Effective friction angle } \\
M & =\text { Critical state stress ratio } \\
M_{c} & =\text { Critical state stress ratio on the compression side } \\
M_{e} & =\text { Critical state stress ratio on the extension side } \\
\chi & =\text { Structure bonding } \\
\chi_{0} & =\text { Initial structure bonding } \\
\xi & =\text { Absolute effectiveness of destructuration hardening } \\
\xi_{d} & =\text { Relative effectiveness of destructuration hardening } \\
\alpha & =\text { Yield surface inclination } \\
\alpha_{0} & =\text { Initial yield surface inclination } \\
k_{0} & =\text { Absolute effectiveness of anisotropy hardening } \\
k_{0} & =\text { Relative effectiveness of anisotropy hardening } \\
& =\text { Coefficient of earth pressure at rest } \\
k_{0} & =
\end{aligned}
$$




$$
\begin{aligned}
C_{k} & =\text { Permeability change index } \\
C_{k}^{*} & =\text { Modified permeability change index } \\
\Delta u_{b} & =\text { Excess pore pressure at base } \\
i & =\text { Hydraulic gradient } \\
R & =\text { Excess pore pressure ratio }
\end{aligned}
$$




\section{INTRODUCTION}

\subsection{GENERAL BACKGROUND}

\subsubsection{CLAYS AND THEIR COMPRESSIBILITY PROPERTIES}

Clay is a fine-grained earth material composed of grain sizes of less than 4 micrometres, as defined in sedimentology. Geologies and colloidal chemists consider a smaller size of 2 micrometres and 1 micrometre respectively (José and Jacinto 2018). The formation of clay is the results of weathering and erosion of rocks mainly containing feldspar. Possible traces of quarts, metal oxides and organic matters also can be found in clay. Clay is an important material in geotechnical engineering owing to its ubiquity in practice. There are also practices to classify clay into a wider category of soft soil, along with other materials such as clayey silt and silty clay (Kempfert and Berhane 2006).

Clay structure consists of platelet particles, and thus can hold a high void ratio and water content. If a moist mass of clay is subject to compression, water and air would be squeezed out with time, a process called consolidation. It is also understood that properties of clay are affected by a combination of particle size and shape, and chemical compositions.

High compressibility is frequently associated with soft clay, contributing to settlement issues of structures built on it. However, predicting settlements of structures built on soft clay foundations still remains a difficult issue. Settlement prediction may become more challenging when structure loads induce significant yielding in soil foundations.

The application of the finite element method (FEM) has been ubiquitous in tackling this issue, given its efficiency and economy. But the accuracy of this numerical approach depends highly on constitutive models due to the fact that many simplifications and assumptions are applied in the development of these models. Most of the proposed soft clay models require soil parameters to be obtained from laboratory tests. Nevertheless, the loading conditions in these tests could be rather different from what soils would experience in the field. As the result, researchers are still looking for appropriate soil models that capture soil response to various loading conditions. 


\subsubsection{ELASTICITY AND PLASTICITY}

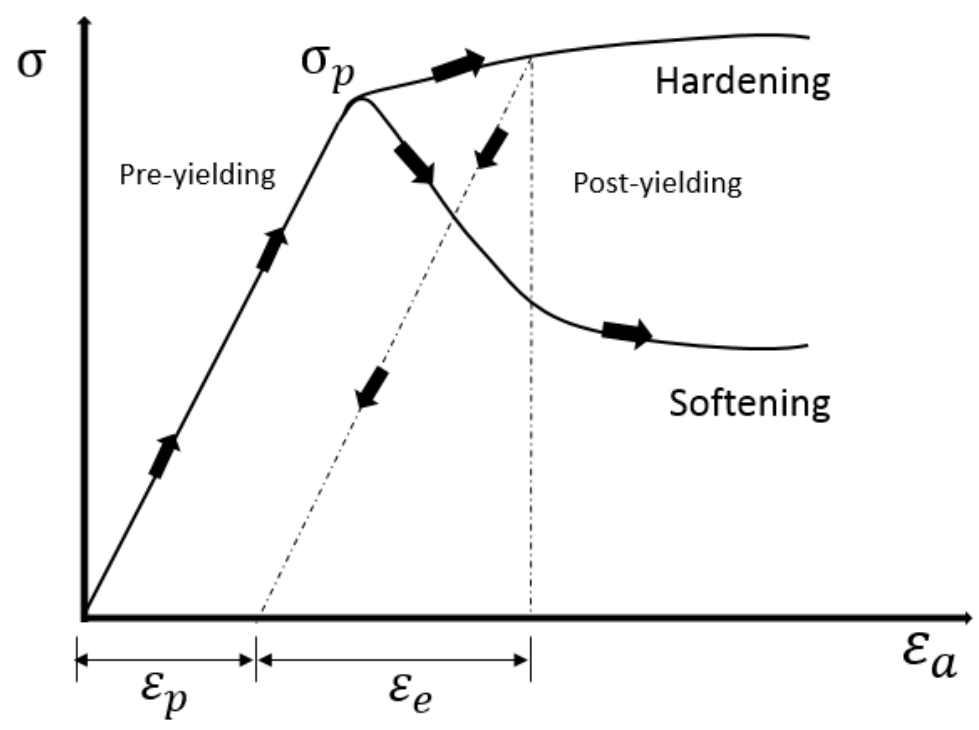

Figure 1.1 Work hardening and softening of soil during unconfined compression tests

To investigate the compressibility of soft clay, one can constitute a stress and strain relationship. There are plenty of experimental studies that provide the evidence of distinct pre-yielding and post-yield responses of soft clay. Figure 1.1 is an illustration of an unconfined compression test on a soft clay sample.

It can be seen that when the soil sample is subject to a pressure or stress lower than its unconfined compression strength $\left(\sigma_{p}\right)$, elastic strains $\left(\varepsilon_{e}\right)$ develops. Elastic strains are considered reversible, meaning that elastic strains are able to recover if the sample is unloaded. However, when the soil sample is loaded beyond its yield stress, plastic strains $\left(\varepsilon_{p}\right)$ emerge. Plastic strains are assumed to be irreversible, indicating the amount of strain that cannot be recovered if the soil sample is unloaded.

It should be noted that Figure 1.1 is an ideal example of soil behavior, which assumes failure strength and yield stress to be the same. In reality, yielding usually occurs ahead of failure, and thus failure strength and yield stress could be quite different. 


\subsubsection{STRAIN HARDENING AND STRAIN SOFTENING PHENOMENA}

In Figure 1.1, one can also notice that yield stresses increase progressively with the plastic straining during the post-yielding phase; this behavior is understood as hardening. Besides, soft soil can also exhibit softening, showing a continuous decrease of compression strength after yielding initiates. As such, stress-strain relationships for soft soil can be proposed by associating elastic and plastic strains with the yield stress evolution. Hardening laws and softening laws are among these attempts, one of which is the volumetric hardening law employed by Modified Cam-Clay (MCC). As suggested by the volumetric hardening law, the increment of yield surface size, $d p_{m}^{\prime}$, defined in a mean effective stress state, progresses with $d \varepsilon_{v}^{p}$, the plastic volumetric strain increment. $d \varepsilon_{v}^{p}$ governs the volume change of a soil sample. It's detailed definition can be found in section 2.1.2.

\subsubsection{SOIL COMPRESSIBILITY AND PLASTIC STRAINS}

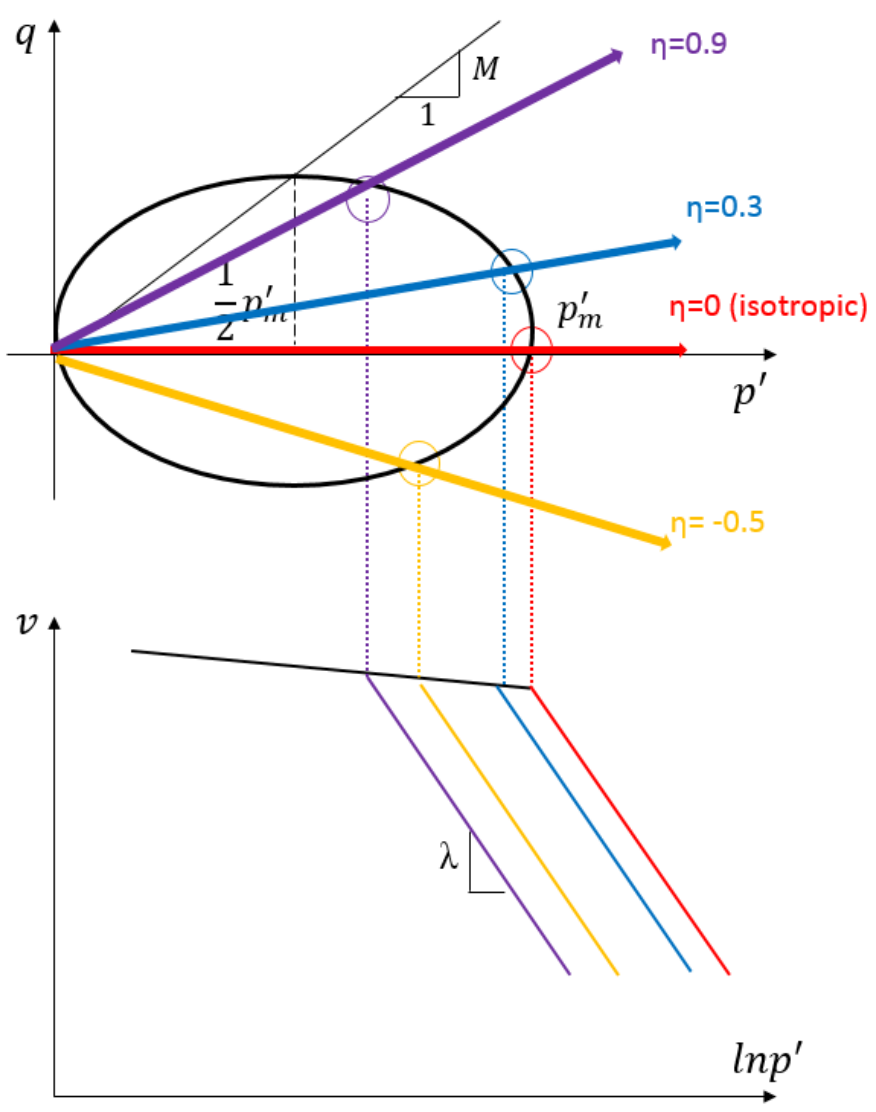

Figure 1.2 Same soil compressibility resulted from various stress ratios modelled by MCC model 
During the plastic straining, the plastic deviatoric strain increment $\left(d \varepsilon_{d}^{p}\right)$, which is related to the shape change of a soil sample, also develops with $\eta$. The definition of $d \varepsilon_{d}^{p}$ can be found in section 2.1.2. However, when describing the yield surface size hardening and softening of a soft clay, the MCC family of critical state models tend to ignore the influence of $d \varepsilon_{d}^{p}$. If the $d \varepsilon_{d}^{p}$, s influence is eliminated, soil will manifest the same compressibility even though different stress conditions are applied. See Figure 1.2 for instance, where an ellipse-shaped yield locus of a soil sample is

presented. Various stress ratios $\eta=\frac{q}{p^{\prime}}$ are applied to the sample. Note that $q=\sigma_{1}^{\prime}-\sigma_{3}^{\prime}$ is the deviatoric stress and $p^{\prime}=\frac{\sigma_{1}^{\prime}+2 \sigma_{3}^{\prime}}{3}$ is the mean effective stress. $\eta=0$ represents the isotropic loading condition where $q=0$, while in any cases that $\eta \neq 0, q$ is involved.

Stress ratios from $\eta=-0.5$ to $\eta=0.9$ are represented by stress paths beginning from the origin, as plotted in Figure 1.2. These paths induce only elastic strains when progressing inside the yield surface. The moment that stress paths intersect the yield surface, plastic strains start to occur. The resulted compression curves are plotted in a well-established $v-\ln p^{\prime}$ space, where $v=1+e$ is the specific volume. The result shows that, if the $d \varepsilon_{d}^{p}$, influence is ignored, various stress paths will result in a group of parallel compression lines, indicating the same soil compressibility.

\subsubsection{SETTLEMENT ISSUES OF WABA DAM BUILT ON CHAMPLAIN SEA CLAY}

Waba dam is an earth-filled structure built on the Champlain Sea clay foundation. Champlain Sea clay is a sensitive marine clay found in eastern Canada. This clay is highly compressible and has entangled many engineers with settlement issues. The open fabric structure of marine clay allows additional share of water to exist between soil particles (Nagaraj et al. 1990). However, the breakdown of this fabric by applying loads can lead to significant volumetric changes, i.e. settlements.

This embankment has settled more than $1.6 \mathrm{~m}$ over the course of 40 years since its construction completed in 1976. It means that the settlement has surpassed $10 \%$ of the dam height at the most of dam sections. As the result, Ontario Power Generation (OPG) has decided to lift the dam up to compensate the height loss. The prediction of long-term settlements of the dam is needed to meet the dam's upgrade need. 


\subsubsection{NEED FOR FURTHER STUDY}

Most of the existing soil models for soft clay ignore the $d \varepsilon_{d}^{p}$, s influence on the compressibility evolution. Some theoretical models to be presented in the next chapter assume that $d \varepsilon_{d}^{p}$ contributes to yield surface size hardening. This assumption makes soil samples less compressible under anisotropic compression than isotropic compression. However, yield surface deviatoric hardening has not been supported by sufficient experimental evidence reported in clays. In some cases, it even contradicts experimental observations (Walker and Raymond 1969, Lade 1976, 1977).

Therefore, the conclusion can be made that the influence of $d \varepsilon_{d}^{p}$ on yield surface size evolution in soft clays is still not well understood or agreed. This issue can be further investigated through experimental approaches. Based on experimental results, a modified yield surface size softening model considering the influence of $d \varepsilon_{d}^{p}$ can be proposed to improve the compressibility prediction for soft clays.

Another purpose of this study is to solve urgent industry needs by investigating the settlement issue of Waba dam. The numerical prediction of the settlement needs to be provided for the further dam lifting design. This will involve a comprehensive laboratory investigation on intact foundation soil samples, in order to yield model parameters for Champlain Sea clay. The modified soil model is expected to be applied in the simulation. The modified model will help better predict the longterm settlement of Waba dam to support the owner to take necessary measures for its safe operation.

\subsection{OBJECTIVES}

The primary objective of this dissertation is to improve the numerical modeling of compressibility issue of soft clay, in particular sensitive clays. Based on the literature review, soft clay tends to exhibit an increased compressibility in response to higher stress ratios. To model this behavior, a modified deviatoric softening law will be developed within the frame work of Yin's elastoviscoplastic (EVP) theory, along with destructuration and yield surface anisotropy of S-CLAY1S. In addition, the newly developed model shall be made executable for sharing with the general geotechnical community to tackle similar geotechnical challenges. The model is first programmed in FORTRAN and implemented in Plaxis V8.2. The development of the new model is expected to 
improve the simulation of soil compressibility of soft clays subject to sophisticated loading conditions.

\subsection{RESEARCH METHODOLOGY}

The method of this research is to propose a modified deviatoric softening law to consider the impact of stress ratio on the compressibility of soft clays. The modified softening law will be combined with other mechanical features of soft clays, including creep, destructuration, and anisotropy. The modified softening law will be developed based on the review of 21 tests on soft clays from published literatures. Then, the verification of the modified model (MEVP-DS) will be done in three phases: Phase I is to simulate 7 published laboratory tests on Finnish clay, Phase II is to simulate 12 consolidation tests on Champlain Sea clay, Phase III is to simulate a real embankment on Champlain Sea clay. The capacity of the new model will be evaluated by comparing its model prediction with test results or field measurements. Its performance over existing models will be evaluated through the comparison on their predictions.

\subsection{OUTLINE OF THE THESIS}

Chapter 2 contains two parts. The first part introduces a number of soil models describing hardening and softening behaviors. Both yield surface models and failure surface models are reviewed and compared. The contribution of $d \varepsilon_{d}^{p}$ to yield surface evolution, as assumed in studied soil models, are summarized. Part two presents a number of published IAC tests performed on Finnish soft clays. The analysis of test results are performed to investigate the dependence of soil compressibility on stress ratios. The purpose of the analysis is to identify the contribution of $d \varepsilon_{d}^{p}$ to yield surface size evolution.

In Chapter 3, a modified deviatoric softening law for soft clays is introduced. Moreover, a new model, named MEVP-DS, has been proposed. MEVP-DS has incorporated the modified softening law, along with viscosity, yield surface anisotropy, and destructuration. Then, MEVP-DS has been programmed in FORTRAN and implemented in Plaxis V8.2. The verification of MEVP-DS has 
been done through the simulation of a number of IAC tests on Finnish clays. The improved prediction by MEVP-DS is demonstrated by comparing with S-CLAY1 and MCC model predictions.

Chapter 4 further validates MEVP-DS model through simulating a number of 1-D consolidation tests conducted on intact Champlain Sea clay samples. The simulated tests include constant rate of strain tests, conventional oedometer tests, and 1-D creep tests. The improvement to the compressibility modeling of Champlain Sea clay by considering deviatoric softening is demonstrated.

Chapter 5 is a case study verification of MEVP-DS application in simulating the long-term settlement behavior of an embankment built on Leda clay foundation in eastern Canada. In this chapter, a 2D plain strain model of a typical cross section of Waba dam is established in Plaxis V8.2. The $70 \mathrm{~m}$ thick deposit has been modeled with MEVP-DS and some reduced cases of MEVP model implemented as user-defined models. The numerical predictions are compared with the field measurements to demonstrate model capacity. Moreover, the contribution of deviatoric softening, creep, destructuration and anisotropy, to the long-term behaviour of Waba Dam foundation is also investigated.

Chapter 6 summarizes the conclusions and main contributions of the study and provide suggestions for future work. 


\section{LITERATURE REVIEW: NUMERICAL MODELING OF SOFT CLAY BEHAVIOUR}

\subsection{FUNNDAMENTAL CONCEPTS}

To assist with reading, this section provides the definitions of some basic concepts and terminologies used throughout the dissertation.

\subsubsection{YIELD SURFACE}

A yield surface of a soil sample is a surface defined in a stress state. A yield surface identifies the boundary between the elastic and plastic behaviour. The stress state is elastic if it's inside the yield surface. Plastic response will develop if the stress state is outside the yield surface. See Figure 2.1 for MCC yield surface, where $M$ is the critical state stress ratio and $p_{m}^{\prime}$ is the size of the yield surface. The yield surface $f$ can be described by the following equation:

$$
f=q^{2}-M^{2}\left(p_{m}^{\prime}-p^{\prime}\right) p^{\prime}=0
$$

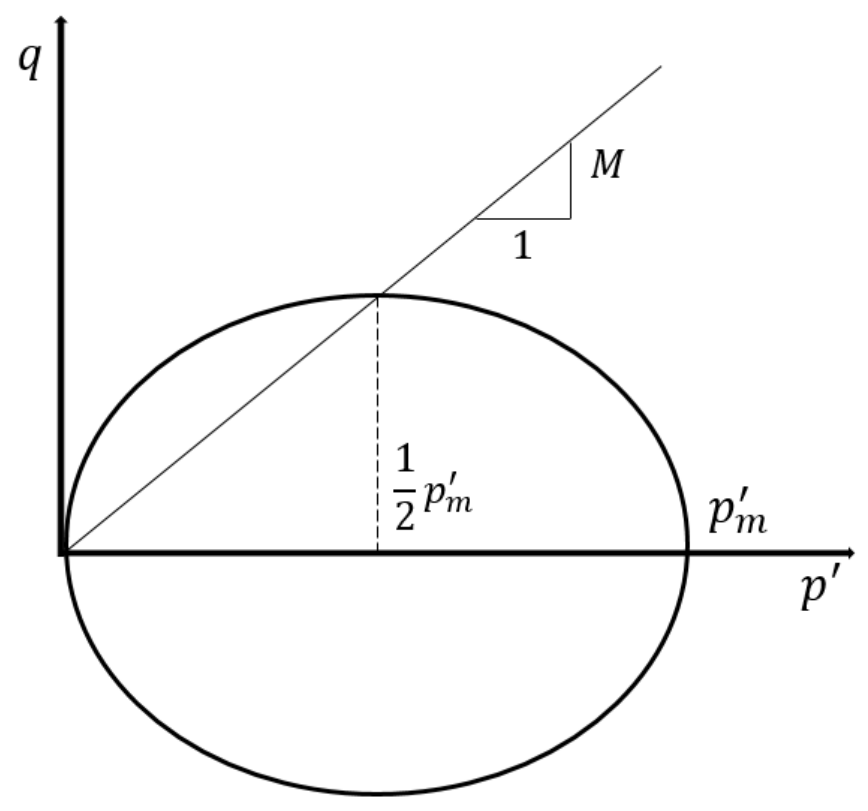

Figure 2.1 Modified Cam-Clay yield surface 
The yield surface used in this thesis follows the MCC ellipse surface defined in a $p^{\prime} \sim q$ space. $p^{\prime}$ is the mean effective stress defined as

$$
p^{\prime}=\frac{\sigma_{x x}^{\prime}+\sigma_{y y}^{\prime}+\sigma_{z z}^{\prime}}{3}
$$

where $\sigma_{x x}^{\prime}, \sigma_{y y}^{\prime}$ and $\sigma_{z z}^{\prime}$ are effective normal stresses at $x, y, z$ directions, respectively. For a conventional axisymmetric triaxial loading condition, $\sigma_{y y}^{\prime}=\sigma_{a}^{\prime}, \sigma_{x x}^{\prime}=\sigma_{z z}^{\prime}=\sigma_{r}^{\prime}$ :

$$
p^{\prime}=\frac{\sigma_{a}^{\prime}+2 \sigma_{r}^{\prime}}{3}
$$

where $\sigma_{a}^{\prime}$ is the effective axial stress and $\sigma_{r}^{\prime}$ is the effective radial stress or cell pressure.

The deviatoric stress $q$ is defined to be

$$
q=\sqrt{\frac{\left(\sigma_{x x}^{\prime}-\sigma_{y y}^{\prime}\right)^{2}+\left(\sigma_{y y}^{\prime}-\sigma_{z z}^{\prime}\right)^{2}+\left(\sigma_{z z}^{\prime}-\sigma_{x x}^{\prime}\right)^{2}}{2}+3\left(\tau_{y z}^{2}+\tau_{z x}^{2}+\tau_{x y}^{2}\right)}
$$

where $\tau_{x y}, \tau_{x y}$ and $\tau_{x y}$ are shear stresses. For a conventional axisymmetric triaxial loading condition:

$$
q=\sigma_{a}^{\prime}-\sigma_{r}^{\prime}
$$

\subsubsection{PlaStiC POTENTIAL}

Plastic potentials of clays are a family of curves to which plastic strain increment vectors are orthogonal. This means that the outward normal to the plastic potential defines the direction of the resultant of plastic strain increments (shown in Figure 2.2). Plastic strain includes two components: $d \varepsilon_{v}^{p}$ and $d \varepsilon_{d}^{p}$. 


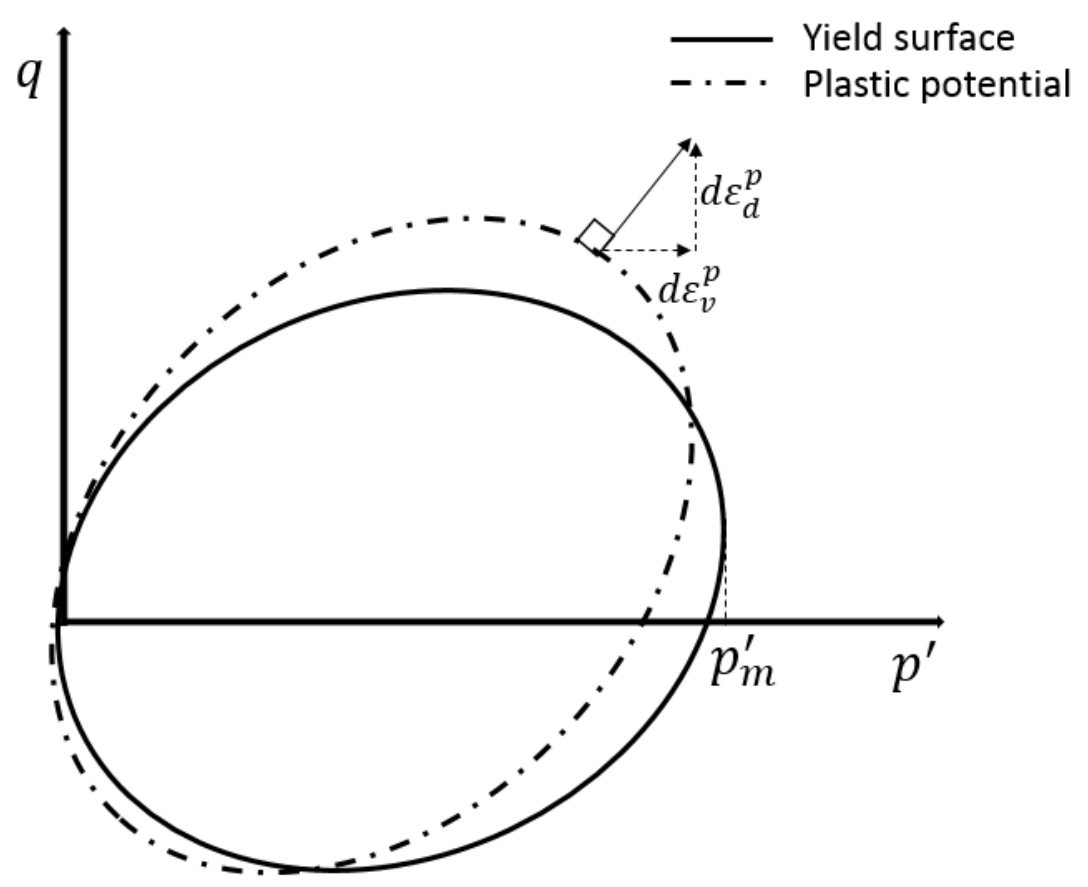

Figure 2.2 Plastic potential and yield surface of soil

The volumetric strain increment is defined as the sum of normal strain increments:

$$
d \varepsilon_{v}=d \varepsilon_{x x}+d \varepsilon_{y y}+d \varepsilon_{z z}
$$

where $\varepsilon_{x x}, \varepsilon_{y y}$ and $\varepsilon_{z z}$ are normal strains at $x, y, z$ directions. For an axisymmetric triaxial soil sample:

$$
d \varepsilon_{v}=\frac{d \varepsilon_{a}+2 \mathrm{~d} \varepsilon_{r}}{3}
$$

where $d \varepsilon_{a}$ is axial strain increment, $d \varepsilon_{r}$ the radial strain increment. The definition of deviatoric strain is

$$
\begin{aligned}
d \varepsilon_{d}= & \frac{1}{3} \sqrt{2\left[\left(d \varepsilon_{y y}-d \varepsilon_{z z}\right)^{2}+\left(d \varepsilon_{x x}-d \varepsilon_{y y}\right)^{2}+\left(d \varepsilon_{z z}-d \varepsilon_{x x}\right)^{2}\right]} \\
& =\frac{+3\left(\gamma_{y z}^{2}+\gamma_{x y}^{2}+\gamma_{x z}^{2}\right)}{}
\end{aligned}
$$

For an axisymmetric triaxial soil sample, deviatoric strain can be simplified as

$$
d \varepsilon_{d}=\frac{2\left(d \varepsilon_{a}-d \varepsilon_{r}\right)}{3}
$$


This is based on assuming $d \varepsilon_{a}=d \varepsilon_{y y}$, and $d \varepsilon_{r}=d \varepsilon_{x x}=d \varepsilon_{z z}$.

\subsubsection{STRESS PATH}

When a soil sample undergoes constant stress state changes, for instance during a triaxial shearing test, the stress path concept can be employed to dynamically locate the stress state. Stress paths are often plotted in $p^{\prime} \sim q$ space, as shown in Figure 2.3. CID and CIU represent the consolidated isotropically drained triaxial compression test and undrained compression test respectively.

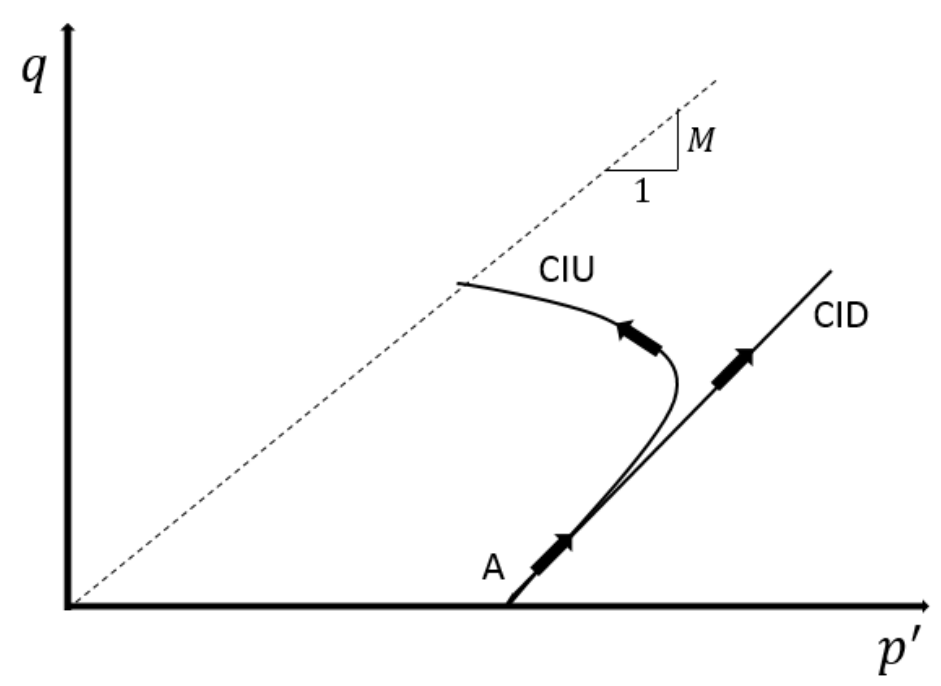

Figure 2.3 Stress paths of soil subject to conventional CIU and CID loading

\subsection{YIELD SURFACE AND FAILURE SURFACE OF SOIL}

It is a common practice to assume that a soil sample possesses two surfaces in $p^{\prime} \sim q$ space, including a failure surface and a yield surface. As shown in Figure 2.4, both surfaces can be subject to hardening (size expansion) and softening (size contraction) depending on individual soil model assumptions. 


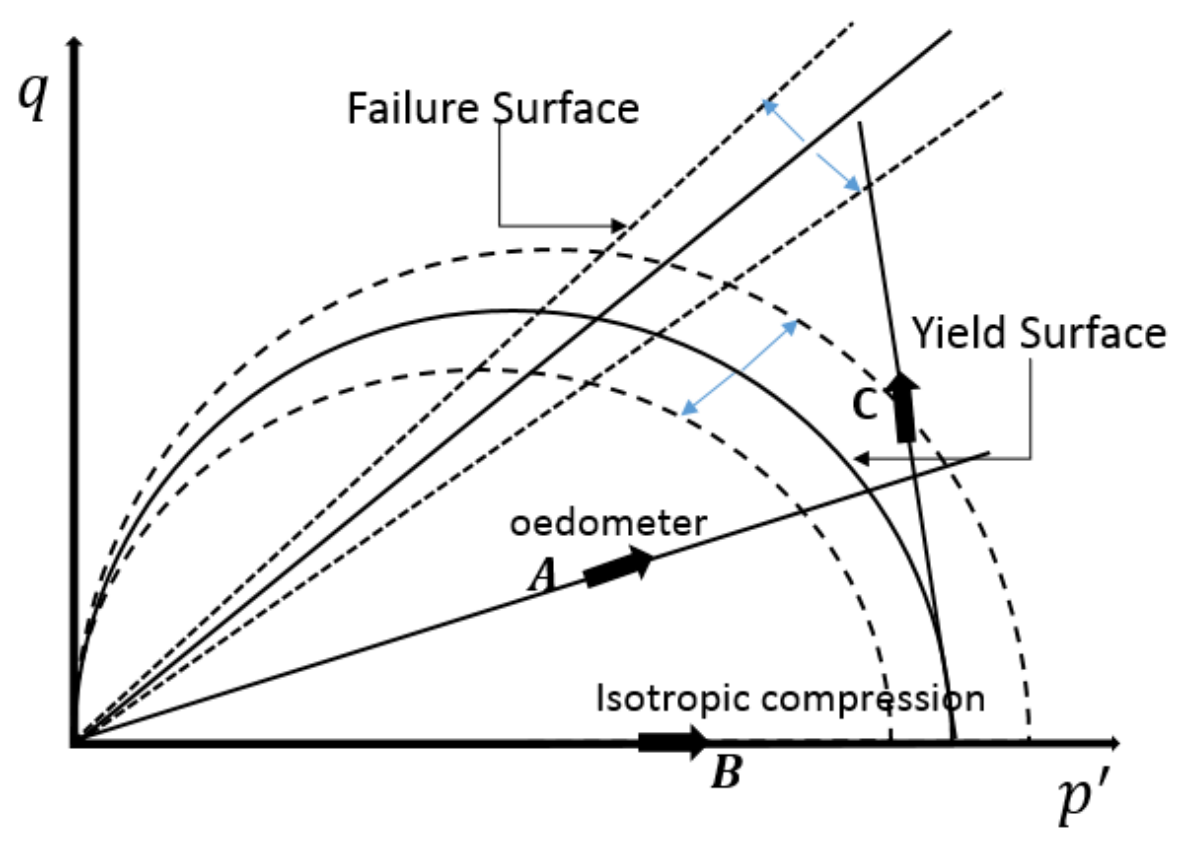

Figure 2.4 Yield surface (cap surface) and failure surface of soil

Examples for yield surface hardening include oedometer or isotropic compression tests where yield surfaces are observed to expand after the pre-consolidation is reached. See Figure 2.4, once the stress path A representing oedometer tests touches the yield surface, plasticity starts to occur and the yield surface expands. Likewise, in the isotropic compression case represented by the stress path B. In some soil models, this yield surface is also called the cap surface. Note that soil samples are not likely to fail during typical consolidation tests mentioned above. The reason is that $\eta$ applied in these tests are usually lower than failure stress ratios of soil samples.

However, during shearing tests such as triaxial tests, soil fails when the applied $\eta$ surpasses the failure stress ratio. For instance, when the stress path $\mathrm{C}$ intersects the failure surface, failure occurs. Consequently, the failure surface may begin to expand or contract, resulting in distinct post-failure shear strength behaviors. These failure surface models are also known as distortional models.

The following sections will present an overview of hardening and softening models for soil. Both yield surface and distortional models will be discussed and compared. 


\subsection{YIELD SURFACE HARDENING MODELS}

\subsubsection{YIELD SURFACE VOLUMETRIC HARDENING MODELS FOR SAND AND CLAY}

Figure 2.5 is presented to explain volumetric hardening law of MCC model. Hardening law basically describes the yield surface increment $d p_{m}^{\prime}$ due to an increase of $d \varepsilon_{v}^{p}$. This assumption has been employed in the Cam-Clay volumetric strain hardening framework (Roscoe and Burland 1968):

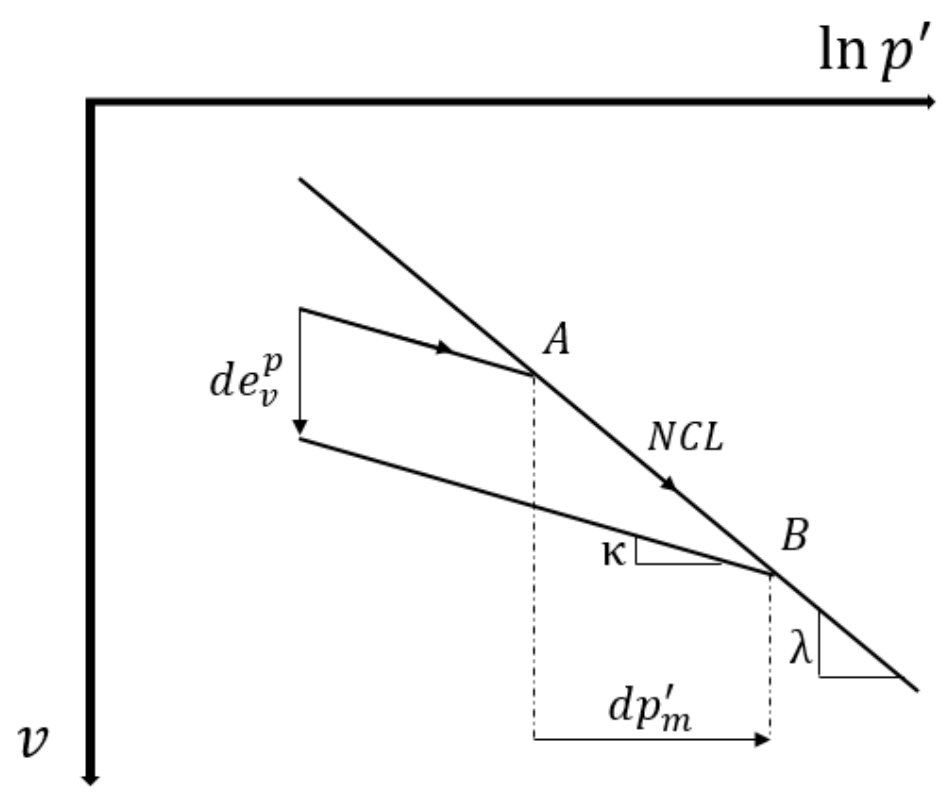

Figure 2.5 Volumetric hardening law of MCC model

$$
d p_{m}^{\prime}=\frac{v p_{m}^{\prime}}{(\lambda-\kappa)} d \varepsilon_{v}^{p}
$$

Equation [2.10] implies that $d p_{m}^{\prime}$ is governed only by $d \varepsilon_{v}^{p}$. When $d \varepsilon_{v}^{p}$ is positive (compression), the yield surface expands (hardening), whereas when $d \varepsilon_{v}^{p}$ is negative (dilation), the yield surface contracts (softening).

Volumetric hardening law can be seen in a vast hierarchy of Cam-Clay family models (Nakai and Matsuoka 1986, Yin and Graham 1999, Dafalias et al. 2006, Karstunen and Koskinen 2008, Suebsuk et al. 2010, Sivasithamparam and Castro 2015). Some other models also use volumetric 
hardening law to describe the expansion of cap surfaces. Examples are the well-established Hardening Soil model (Schanz et al. 1999) and Mortara’s model (Mortara 2015).

\subsubsection{YIELD SURFACE BIASED HARDENING MODELS FOR SAND AND CLAY}

Biased hardening models assume that both $d \varepsilon_{v}^{p}$ and $d \varepsilon_{d}^{p}$ contribute to $d p_{m}^{\prime}$, with their contributions distinguished by a weight factor or different mathematical forms. There exists a variety of biased hardening models for cohesionless soils (Nova and Wood 1978, Krenk 2000, Chang and Hicher 2005, Yao et al. 2008, Lashkari 2010) as opposed to cohesive soils (Collins and Kelly 2002, Yao et al. 2008).

Krenk (2000) introduces the following biased work hardening law:

$$
d p_{m}^{\prime}=\frac{v}{\lambda-\kappa}\left(p^{\prime} d \varepsilon_{v}^{p}+w s_{i j} d \varepsilon_{i j}^{p}\right)
$$

where $w$ is a non-dimensional weight parameter, $s_{i j}=\sigma_{i j}-p^{\prime} \delta_{i j}$ is the deviatoric stress, and $d \varepsilon_{i j}^{p}$ is the plastic shear strain increment. This equation can be restructured as:

$$
d p_{m}^{\prime}=\frac{v p^{\prime}}{\lambda-\kappa}\left(d \varepsilon_{v}^{p}+w \eta d \varepsilon_{i j}^{p}\right)
$$

where $\eta=\frac{s_{i j}}{p^{\prime}}$ the stress ratio. Note that this is different from the stress ratio $\eta$ defined before, as $s_{i j}=\sqrt{\frac{2}{3}} q$. The similar biased work hardening law in [2.12] for describing yield surface size evolution has been also adopted by Liu et al. (2013).

$$
d p_{m}^{\prime}=\frac{v p^{\prime}}{\lambda-\kappa}\left(d \varepsilon_{v}^{p}+\alpha \eta d \varepsilon_{i j}^{p}\right)
$$

where the weight parameter $\alpha$ is used in lieu of $w . \alpha$ is the anisotropic inclination of yield surface. When $\alpha=0$ (no anisotropy) or $\eta=0$ (isotropic deformation), the hardening law in Equation [2.13] is reduced to the volumetric hardening law in Equation [2.10].

It is worth noted that the above discussed biased work hardening laws in fact tend to assume yield surface deviatoric hardening. It indicates that $d \varepsilon_{d}^{p}$ contributes to yield surface size hardening, where soil samples will become less compressible under anisotropic compressions than isotropic compressions. However, deviatoric hardening has yet been demonstrated by sufficient 
experimental observations in soft clays. In some cases, it even contradicts experimental observations (Walker and Raymond 1969, Lade 1976, 1977).

\subsection{DISTORTIONAL HARDENING AND SOFTENING MODELS}

Distortional hardening and softening are associated with shear strength increase or decrease during a shearing test after the peak shear strength is reached. It can be noticed that, $d \varepsilon_{d}^{p}$ is the dominant

factor in many distortional models to be discussed this section. In these models, $d \varepsilon_{d}^{p}$ is linked to shear strength or shear strength parameter evolution, such as friction angles or failure stress ratios.

\subsubsection{DISTORTIONAL MODELS FOR SAND}

One of the distortional models involving the direct description of shear strengths was proposed by Lade (1977). In this model for granular soil, $q$ can be hardened or softened after the peak strength is surpassed:

$$
f=q-A \frac{B\left(\varepsilon_{d}^{p}\right)^{2}+\varepsilon_{d}^{p}}{1+\left(\varepsilon_{d}^{p}\right)^{2}}=0
$$

where $A$ and $B$ are two constants, and $\varepsilon_{d}^{p}$ is calculated by

$$
\begin{gathered}
\varepsilon_{d}^{p}=\int d \varepsilon_{d}^{p} \\
d \varepsilon_{d}^{p}=\left\{\frac{2}{3} d \varepsilon_{i j}^{p} d \varepsilon_{i j}^{p}\right\}^{2}=\frac{\sqrt{2}}{3}\left\{\left(d \varepsilon_{1}^{p}-d \varepsilon_{2}^{p}\right)^{2}+\left(d \varepsilon_{2}^{p}-d \varepsilon_{3}^{p}\right)^{2}+\left(d \varepsilon_{3}^{p}-d \varepsilon_{1}^{p}\right)^{2}\right\}^{1 / 2}
\end{gathered}
$$

This idea of curve-fitting shear strength directly with strain components can be also seen in (Prévost and Höeg 1975, Banimahd and Woodward 2006).

Wood and Belkheir (1994) managed to model shear strength hardening when the sand is sheared at the "wet" side through linking the peak stress ratio to a state parameter. Softening is assumed to happen at the "dry" side

$$
\frac{\eta}{M_{p}}=\frac{\varepsilon_{q}}{\left(B+\varepsilon_{q}\right)}
$$


where $M_{p}$ is the peak stress ratio, $\varepsilon_{q}$ is the shear strain, and $B$ is a constant. In this equation, $\eta$ approaches to the asymptotic value of $\frac{M_{p}}{B} . M_{p}$ is defined by the critical state stress ratio at compression side $M_{c}$, a state parameter $\psi$, and a constant $k$ by:

$$
M_{p}=M_{c}-k \psi
$$

State parameter $\psi$ is given by:

$$
\psi=v-v_{c}=v+\lambda \ln p^{\prime}-\Gamma
$$

where $v_{c}$ is the specific volume at the critical state, and $\Gamma$ is the initial specific volume of critical state at unit mean effective stress $(1 \mathrm{kPa})$.

A similar approach of associating stress ratio or friction angle evolution with plastic strain components for granular material has been also employed by many researchers, such as Lade (1977), Mortara (2015), Chen and Abousleiman (2018).

\subsubsection{DISTORTIONAL MODELS FOR CLAY}

The distortional models for clay share the similar pattern with sand models. One of the examples by Zabala and Alonso (2011) is given as:

$$
\phi^{\prime}=\phi_{r e s}^{\prime}+\left(\phi_{p e a k}^{\prime}-\phi_{r e s}^{\prime}\right) e^{-\eta d \varepsilon_{d}^{p}}
$$

where $\phi^{\prime}$ is the effective friction angle, $\phi_{\text {res }}^{\prime}$ is the residual effective friction angle, $\phi_{\text {peak }}^{\prime}$ is the peak effective friction angle, and $\eta$ is a constant. The likewise approach has been employed by other models (Potts et al. 1997, Schanz et al. 1999, Vatsala et al. 2001, Einav and Randolph 2005, Conte et al. 2013).

It is worth noted that for critical state models, $M$ is not allowed to evolve after the failure is reached. As such, MCC is not able to accommodate hardening or softening after failure. However, one can still approach this problem by describing softening of the structured yield surface. These destructuration models are tailored to model sensitive clays with distinct behaviors between undisturbed and remolded soil samples. 


\subsection{YIELD SURFACE DESTRUCTURATION THEORIES}

It has been recognized that considering soil structure is essential in determining mechanical behaviors of soft sensitive clays (Mitchell 1970, Lo and Morin 1972, Burland 1990, Leroueil and Vaughan 1990, Rouainia and D 2000, Hong et al. 2007). The term "soil structure" is defined as the arrangement and bonding of soil constituents. It encompasses all features of the soil that cause its mechanical behavior to be different from that of the corresponding reconstituted soil (Burland 1990, Calalisto and Calabresi 1998, Cucovillo and Coop 1999, Cotecchia and Chandler 2000, Gasparre et al. 2007).

To describe the soil structure, a very common approach is to introduce a bonding parameter. In many models available (Koskinen et al. 2002, Baudet and Stallebrass 2004, Callisto and Rampello 2004, Panayides et al. 2012, Liu et al. 2013), bonding is defined as the ratio of the pre-consolidation pressure of an undisturbed clay to the pre-consolidation pressure of the corresponding reconstituted clay at the same strain level.

Under loading conditions, bonding degradation is initiated in response to plastic strain increments: $d \varepsilon_{v}^{p}$ and $d \varepsilon_{d}^{p}$. The loss of bonding results in transformation of an undisturbed condition into a remoulded one. Various mathematical expressions have been proposed in destructuration models to associate plastic strain increments with bonding degradations. They are understood as destructuration laws, which are different in various destructuration models.

In following sections, some well-established destructuration models will be presented and their differences will be discussed.

\subsubsection{KOSKINEN'S DESTRUCTURATION MODEL}

Koskinen's destructuration model (Koskinen et al. 2002) has been later developed into the wellknown SCLAY1-S and EVP-SCLAY1S family of models, with EVP-SCLAY1S incorporated into Plaxis 2018 as the embedded model. Structure bonding is assumed to grant an additional yield surface size to the undisturbed clay compared to the remoulded clay. The initial bonding is described using a scalar state variable $\chi_{0}$, see Figure 2.6. $\left(1+\chi_{0}\right)$ denotes the initial ratio of static yield surface size $p_{m}^{\prime}$ and the intrinsic yield surface size $p_{m 0}^{\prime}$ corresponding to undisturbed and remoulded samples respectively. 


$$
p_{m}^{\prime}=(1+\chi) p_{m 0}^{\prime}
$$

In Karstunen's model, both visco-plastic volumetric strain increment, $d \varepsilon_{v}^{v p}$, and the visco-plastic deviator strain increment, $d \varepsilon_{d}^{v p}$, contribute to the bonding degradation, i.e., causing $\chi$ to decrease. The destructuration rate is governed by two parameters: $\xi$, the absolute effectiveness of destructuration hardening and $\xi_{d}$, the relative effectiveness of destructuration hardening.

$$
d \chi=-\xi \cdot \chi \cdot\left(\left|d \varepsilon_{v}^{v p}\right|+\xi_{d} \cdot d \varepsilon_{d}^{v p}\right)
$$

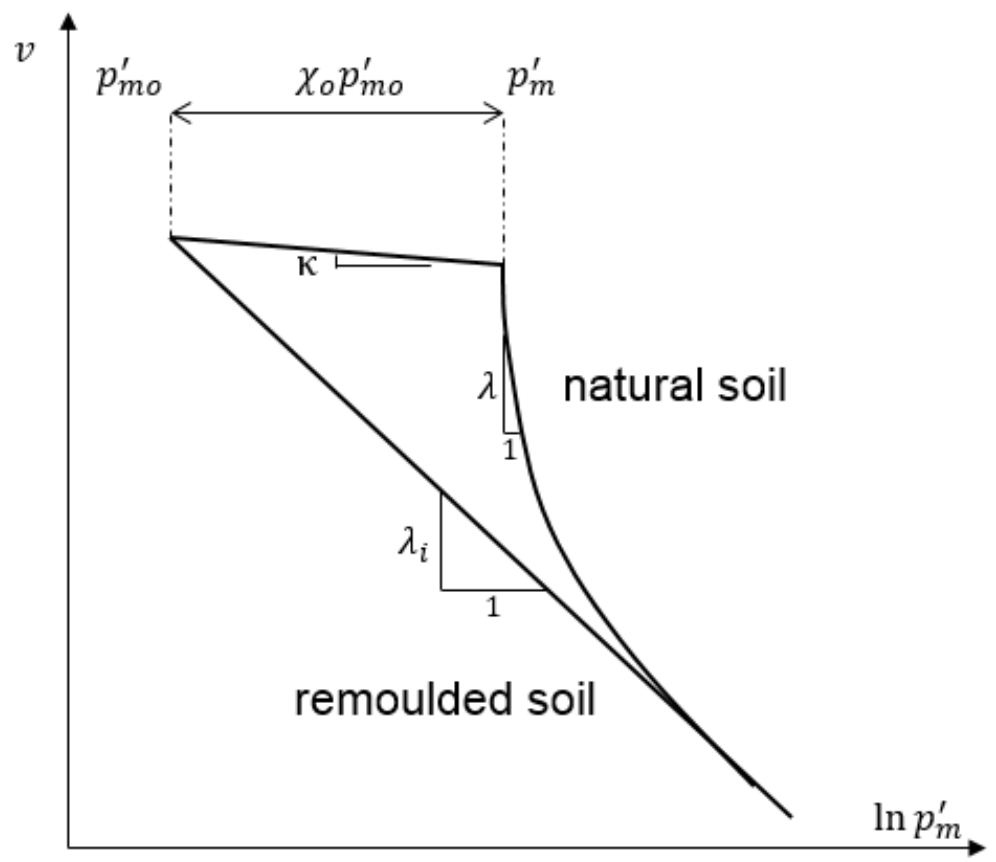

Figure 2.6 Koskinen's definition of soil structure in $v-\ln p^{\prime}$ space (Koskinen et al. 2002)

\subsubsection{BAUDET AND STALLEBRASS'S DESTRUCTURATION MODEL}

Using the similar bonding concept for soil structure descriptions, Baudet and Stallebrass's destructuration model (Baudet and Stallebrass 2004) introduces a third yield surface, the ultimate structured surface, to which the structured yield surface approaches.

$$
\chi=\chi_{f}+\left(\chi_{0}-\chi_{f}\right) E X P\left|-\frac{b}{\lambda-\kappa} \varepsilon_{d}\right|
$$


where $\chi_{f}$ denotes the ultimate natural bonding at a very high stress level, $b$ is a parameter to govern the destructuration rate, and $\varepsilon_{d}$ denotes the damage strain defined as

$$
\varepsilon_{d}=\sqrt{(1-A) d \varepsilon_{v}^{p^{2}}+A d \varepsilon_{d}^{p^{2}}}
$$

where $A$ is a weighting parameter. This type of destructuration laws considering three yield surfaces has been applied in many models (Callisto and Calabresi 1998, Hinchberger et al. 2010, Panayides et al. 2012, Liu et al. 2013, Yang et al. 2015).

\subsubsection{LIU'S DESTRUCTURATION MODEL}

Liu's destructuration model (Liu and Carter 2000a, 2002, 2003) also employs the intrinsic yield surface as the reference to locate the structured yield surface. Unlike the previously discussed models where bonding provides extra sizes to the intrinsic surface, the bonding defined in Liu's model grants an additional void ratio $\Delta e$.

$$
e=e^{*}+\Delta e
$$

where $e^{*}$ denotes the void ratio of remolded soils. With the increase of effective stresses, $\Delta e$ reduces to the level when the natural soil compression curve converges with the remolded compression line, as seen in Figure 2.7.

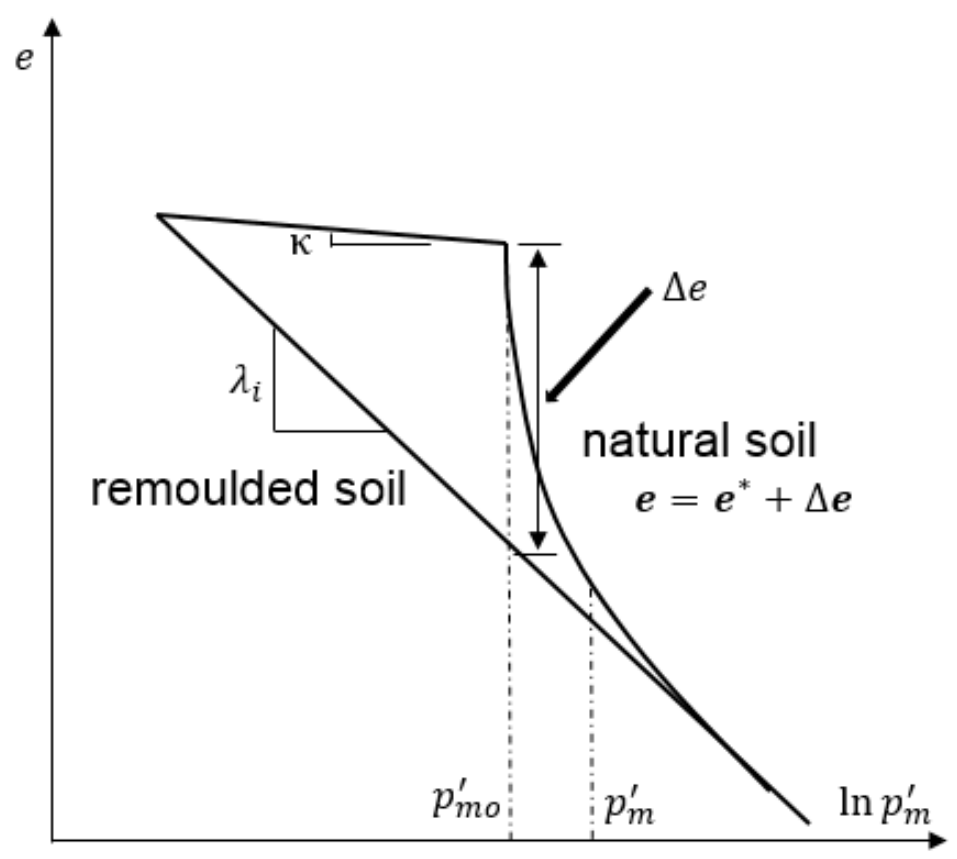


Figure 2.7 3-D case of Liu's destructuration model (Liu and Carter 2000b)

The reduction of $\Delta e$ with respect to stress increase follows the exponential rule:

$$
\Delta e=\Delta e_{i}+\left(\frac{p_{m o}^{\prime}}{p_{m}^{\prime}}\right)^{b}
$$

where $b$ is a parameter governing the rate of destructuration. The destructuration law employs the MCC volumetric hardening law for the remold soil part. For the natural soil part, $\frac{\eta}{M-\eta}$ is added consider stress ratio effect to the compression.

$$
d \varepsilon_{v}^{p}=(\lambda-\kappa) \frac{d p_{m}^{\prime}}{(1+e) p_{m}^{\prime}}+b \Delta e\left(1+\frac{\eta}{M-\eta}\right) \frac{d p_{m}^{\prime}}{(1+e) p_{m}^{\prime}}
$$

\subsection{YIELD SURFACE ANISOTROPY THEORIES}

Isotropic yield surface soil models such as MCC has been proved to provide a good prediction of remould soft clay behavior. However, natural soils sedimented in an anisotropic stress condition, Ko consolidation. Accordingly, soil fabric and particle contacts of naturally deposited soils are distinct from the isotropic condition. Ignoring the fabric anisotropy would result in the predictions that do not match with experimental observations in term of settlement, shear strength, and permeability. Rotational anisotropy and multi-laminate theories are two of many methods to describe the yield surface anisotropy. The details of these two family of models will be briefed below.

\subsubsection{ROTATIONAL ANISOTROPY THEORIES}

Experimental studies indicate that yield surfaces of naturally deposited clays are inclined ellipses (Tavenas and Leroueil 1977, Graham et al. 1984), with centerlines oriented around $K_{0}$ stress paths in a $p^{\prime} \sim q$ space, as seen in Figure 2.8. The constitutive modeling of anisotropy has then focused on describing the anisotropy inclination.

These models are known as rotational or kinematic hardening models. Some early anisotropic models have been proposed to consider the inclined yield surface, with the centreline oriented to the $K_{0}$ line (Sekiguchi and Ohta 1977, Mouratidis and Magnan 1982). However, these models 
assume a fixed surface inclination that stays unchanged during plastic straining. To tackle this issue, some modified models (Wheeler 1997, Dafalias et al. 2002) accommodate an evolving yield surface influenced by $d \varepsilon_{v}^{p}$ and $d \varepsilon_{d}^{p}$. Details of these models are introduced as follows.

Firstly, by introducing a stress ratio related parameter $\alpha$, an energy dissipation equation has been proposed by some researchers (Dafalias 1986):

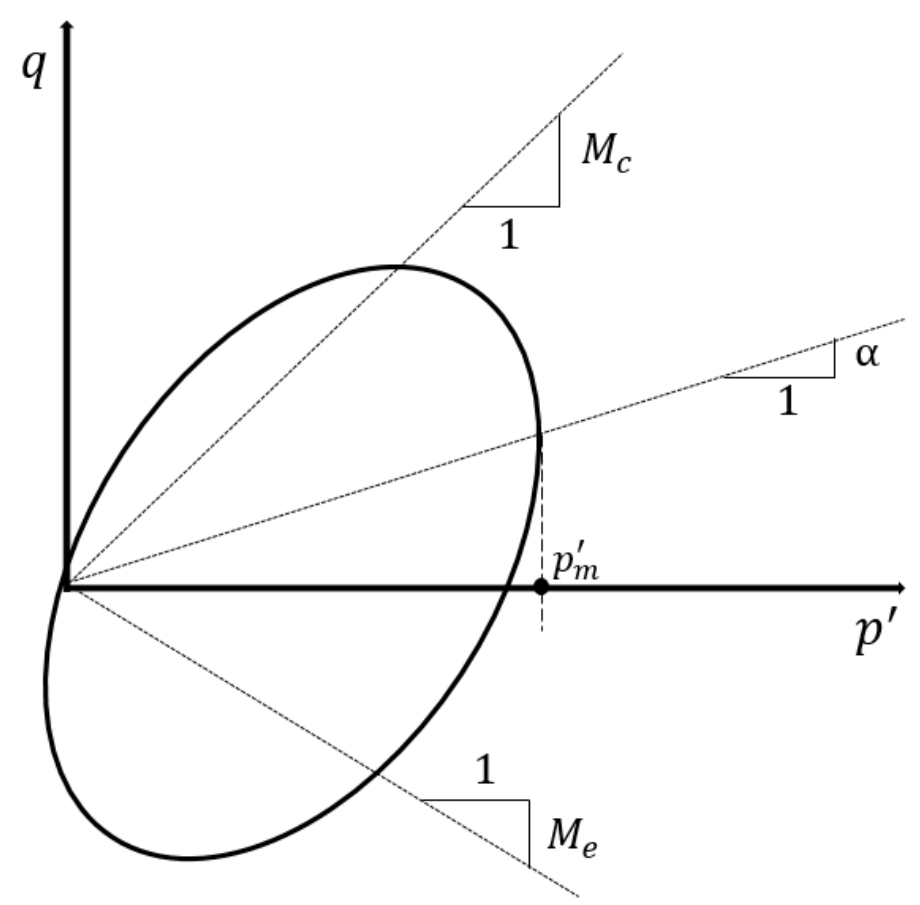

Figure 2.8 The inclined yield surface assumed in rotational anisotropy models (Wheeler et al. 2003)

$$
p \dot{\varepsilon}_{v}^{p}+q \dot{\varepsilon}_{q}^{p}=p \sqrt{\left(\dot{\varepsilon}_{v}^{p}\right)^{2}+\left(M \dot{\varepsilon}_{q}^{p}\right)^{2}+2 \alpha \dot{\varepsilon}_{v}^{p} \dot{\varepsilon}_{q}^{p}}
$$

where $\dot{\varepsilon}_{v}^{p}$ is the plastic volumetric strain rate and $\dot{\varepsilon}_{q}^{p}$ is the plastic deviatoric strain rate.

Then, the plastic potential can be obtained by the integral of the energy equation. The plastic potential appears to be an inclined ellipse with the rotation governed by $\alpha$ and the size controlled by $p_{m}^{\prime}$.

$$
g=\left(q-\alpha p^{\prime}\right)^{2}-\left(M^{2}-\alpha^{2}\right)\left(p_{m}^{\prime}-p^{\prime}\right) p^{\prime}=0
$$


Assuming an associated law, the yield surface $f$ shares the same expression with $g$

$$
f=g=\left(q-\alpha p^{\prime}\right)^{2}-\left(M^{2}-\alpha^{2}\right)\left(p_{m}^{\prime}-p^{\prime}\right) p^{\prime}=0
$$

What's more, an evolution law for $\alpha$ has been proposed. $\alpha$ is assumed to approach $\eta / x$ :

$$
\begin{gathered}
\dot{\alpha}=\langle L\rangle \bar{\alpha}=\langle L\rangle\left(\frac{1+e_{0}}{\lambda-\kappa}\right) C\left(\frac{p^{\prime}}{p_{m}^{\prime}}\right)^{2}\left|\frac{\partial g}{\partial p^{\prime}}\right| \eta-x \alpha \mid\left(\alpha^{b}-\alpha\right) \\
\alpha^{b}=\left\{\begin{array}{cl}
M_{c}, & \eta / x>\alpha \\
-M_{e}, & \eta / x<\alpha
\end{array}\right.
\end{gathered}
$$

where $\langle L\rangle$ is a scalar function, $x$ is a constant, $M_{e}$ is the critical state ratio on the extension side. In this evolution law, $\alpha$ evolution is dependent on $d \varepsilon_{v}^{p}$ only and stops evolving at the critical state in which $\frac{\partial g}{\partial p^{\prime}}=0$, and it is demanded that $|\alpha|<M$. This has become the later Saniclay model (Dafalias et al. 2002, 2006).

Wheeler et al. (2003) argued that both $d \varepsilon_{v}^{p}$ and $d \varepsilon_{d}^{p}$ should contribute to $\alpha$ evolution. He modified the evolution law based on experimental observations of Otaniemi clay in Finland, which has become the later S-CLAY1 model:

$$
d \alpha=\omega\left[\left(\frac{3 \eta}{4}-\alpha\right)\left\langle d \varepsilon_{v}^{p}\right\rangle+\omega_{d}\left(\frac{\eta}{3}-\alpha\right)\left|d \varepsilon_{d}^{p}\right|\right]
$$

where $\omega$ is the absolute effectiveness of anisotropic hardening, and $\omega_{d}$ is the relative effectiveness of anisotropic hardening. The application of this model could be seen in a wide range of embankments modeling on soft clay foundations (Yin and Karstunen 2008, Yildiz et al. 2009, Yin et al. 2010, Sivasithamparam et al. 2015, Yildiz and Uysal 2016).

\subsubsection{MULTI-LAMINATE ANISOTROPY THEORIES}

The multi-laminate framework for rocks was arguably firstly published in Zienkiewicz and Pande (1977). The theory has been applied to soils in Pande and Sharma (1983) as a mathematically simple way of introducing anisotropy.

In this theory, the inherent anisotropy is presented by a set of scalar parameters which are explicit functions of a mixed invariant of stress and microstructure tensors (Pietruszczak and Pande 2001). The multi-laminate framework has then been extended to consider destructuration (Cudny and 
Vermeer 2004), and incorporated into a general constitutive model to for normally consolidated soils (Schweiger et al. 2009). The theory has been applied to the simulation of an embankment on a soft clay foundation in Finland (Cudny 2011).

\subsection{COMPRESSIBILITY OF FINNIFHS SOFT CLAYS SUBJECT TO IAC TESTS}

\subsubsection{INTRODUCTION TO INCREMENTAL ANISOTROPIC CONSOLIDATION TESTS}

This section presents a review on published results of incremental anisotropic consolidation (IAC) tests conducted on Finnish soft clays. Both remolded soft clays (Karstunen and Koskinen 2008), and undisturbed soft clays (Wiltafsky et al. 2002, Zentar et al. 2002, Wheeler et al. 2003) are included in the analysis. The purpose of this section is to exhibit stress ratios' effects on soil compressibility.

During IAC tests, incremental loads were applied at daily intervals of 24 hrs to samples, with a constant stress ratio employed at each loading stage. A typical test contains a loading-unloadingreloading cycle. A small stress increment of 2-5 $\mathrm{kPa}$ was selected in these tests in an attempt to maintain a low excess pore pressure level. However, the practice has tremendously prolonged the test duration, resulting in on average eight weeks to complete each test.

Figure 2.9 shows two examples of these incremental consolidation tests. Figure 2.9 (a) shows a typical compression test, denoted by "CAD", where $\eta_{1}$ represents the stress ratio applied at the first loading stage and $\eta_{2}$ for the second stage.

Figure 2.9 (b) presents another test where the sample was under extension in one of the loading stages, denoted as "CAE". This test involves a negative stress ratio $\eta_{1}=-0.59$ in the first stage, while the loading was switched from extension to compression during the reloading stage. The negative sign here indicates the applied confining pressure is higher than vertical pressure $(q=$ $\left.\sigma_{y}^{\prime}-\sigma_{x}^{\prime}<0\right)$. 
(a)

Mean effective stress, $\mathrm{p}^{\prime}(\mathrm{kPa})$

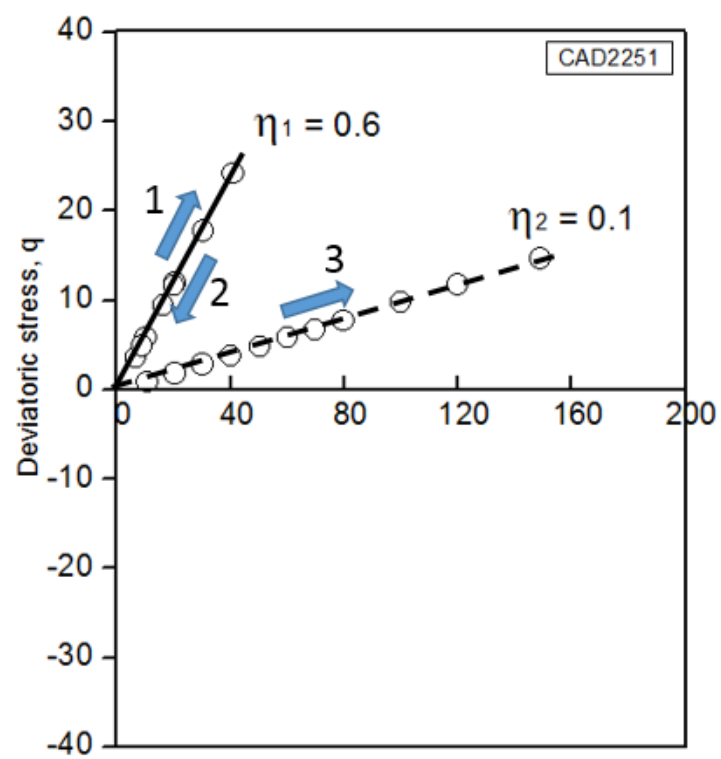

(b)

Mean effective stress, $\mathrm{p}^{\prime}(\mathrm{kPa})$

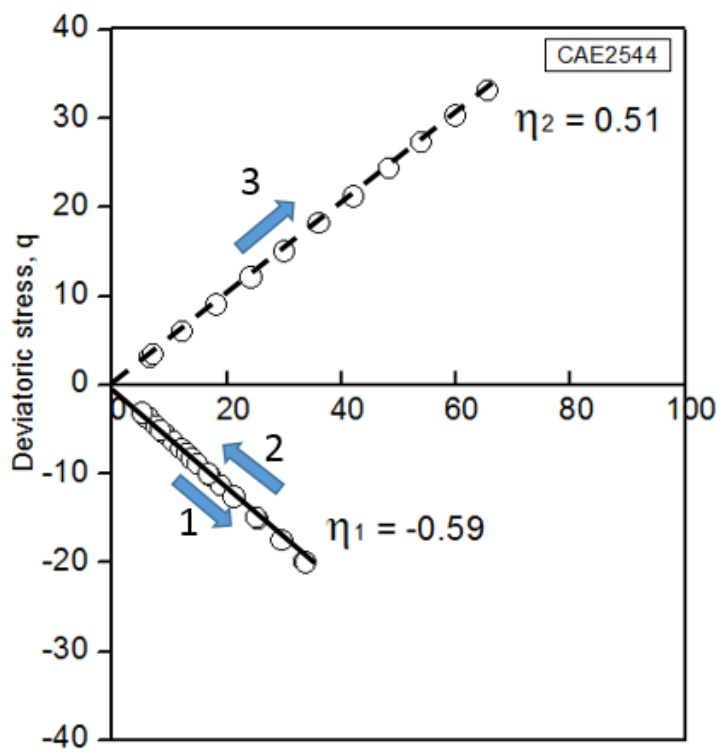

Figure 2.9 Stress paths used in typical IAC tests

\subsubsection{ENGINEERING BACKGROUND OF FINNISH MARINE CLAYS}

The studied Finnish soft clays of this section are from a variety of locations, some of which are Otaniemi clay, Murro clay, and POKO clay. Some Italian Vallericca clays and Canadian Champlain Sea clay are also studied. The focus of this dissertation will be on Otaniemi clay and Murro clay, given the abundance of test data extracted.

Murro clay is a black silty clay found in western coast of Finland, located at the Gulf of Bothonia, see Figure 2.10. The clay deposit was sedimented in brackish water during the postglacial period. Murro clay has been observed to be predominantly normally consolidated, with clay-size fraction of $30 \%$. The black color of Murro clay is due to the presence of sulfur. Its water content is found to be somewhere ranging from $65 \%$ to $100 \%$. The plastic index $I_{p}$ varies from 31 to 66 . The typical sensitivity of Murro clay is around 7 (Karstunen and Yin 2010).

Otaniemi clay is a soft clay found in southern Finland at the shore of the Gulf of Finland, bordering Helsinki, also seen in Figure 2.10. Otaniemi clay has a high clay-sized fraction of $78 \%$ and is mainly composed of illite. The clay samples were extracted undisturbed by Norwegian and Swedish piston samplers. The clay has shown to possess a natural water content of higher than 
90\%. A sensitivity ranging from 7 to14 has been reported for this clay (Wheeler et al. 2003).

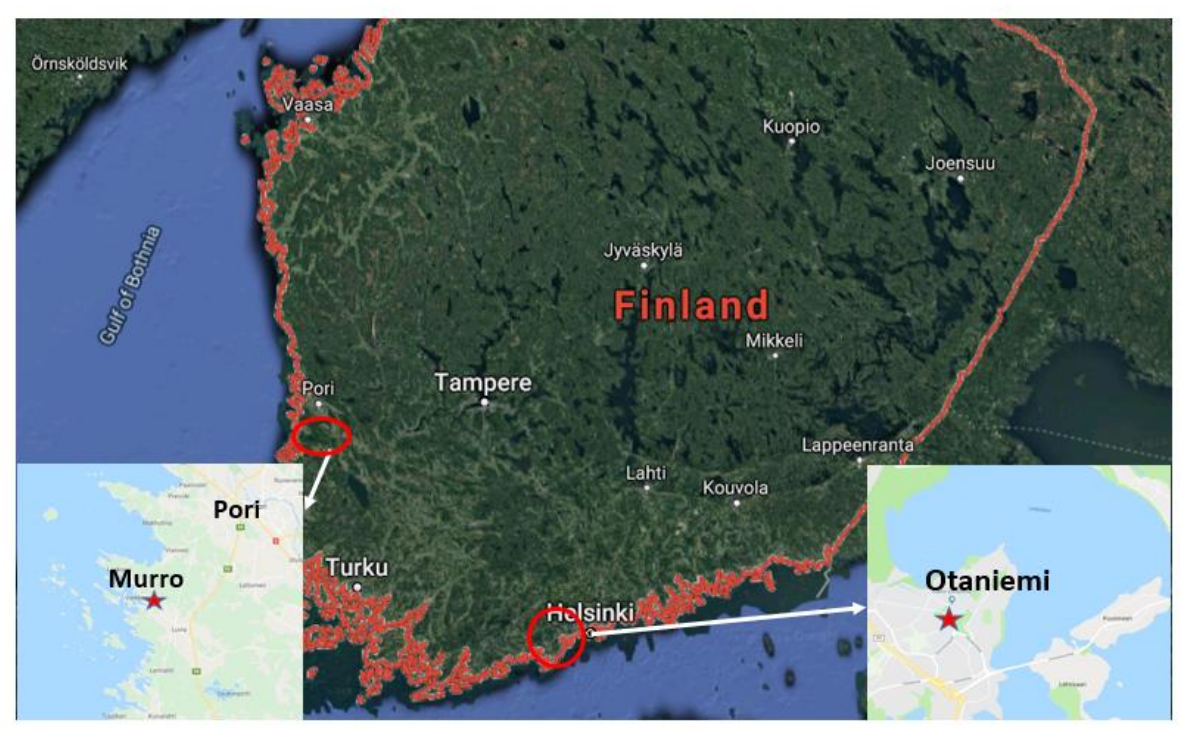

Figure 2.10 Location of Murro and Otaniemi, Finland

\subsubsection{EXPERIMENTAL RESULTS OF IAC TESTS ON REMOLDED MURRO CLAY}

Some results of IAC tests on remolded Murro clay (Karstunen and Koskinen 2008) can be found in Figure 2.11. The samples were fully remolded before finishing primary consolidation in a onedimensional condition at a load of $15 \mathrm{kPa}$. Then, the samples were transferred into a triaxial cell to consolidate at a designed initial stress ratio of $\eta_{0}=0.65$ to reproduce the in situ state. After the initial consolidation was complete, the samples were unloaded to a low stress level close to $10 \mathrm{kPa}$ before the IAC was commenced. 

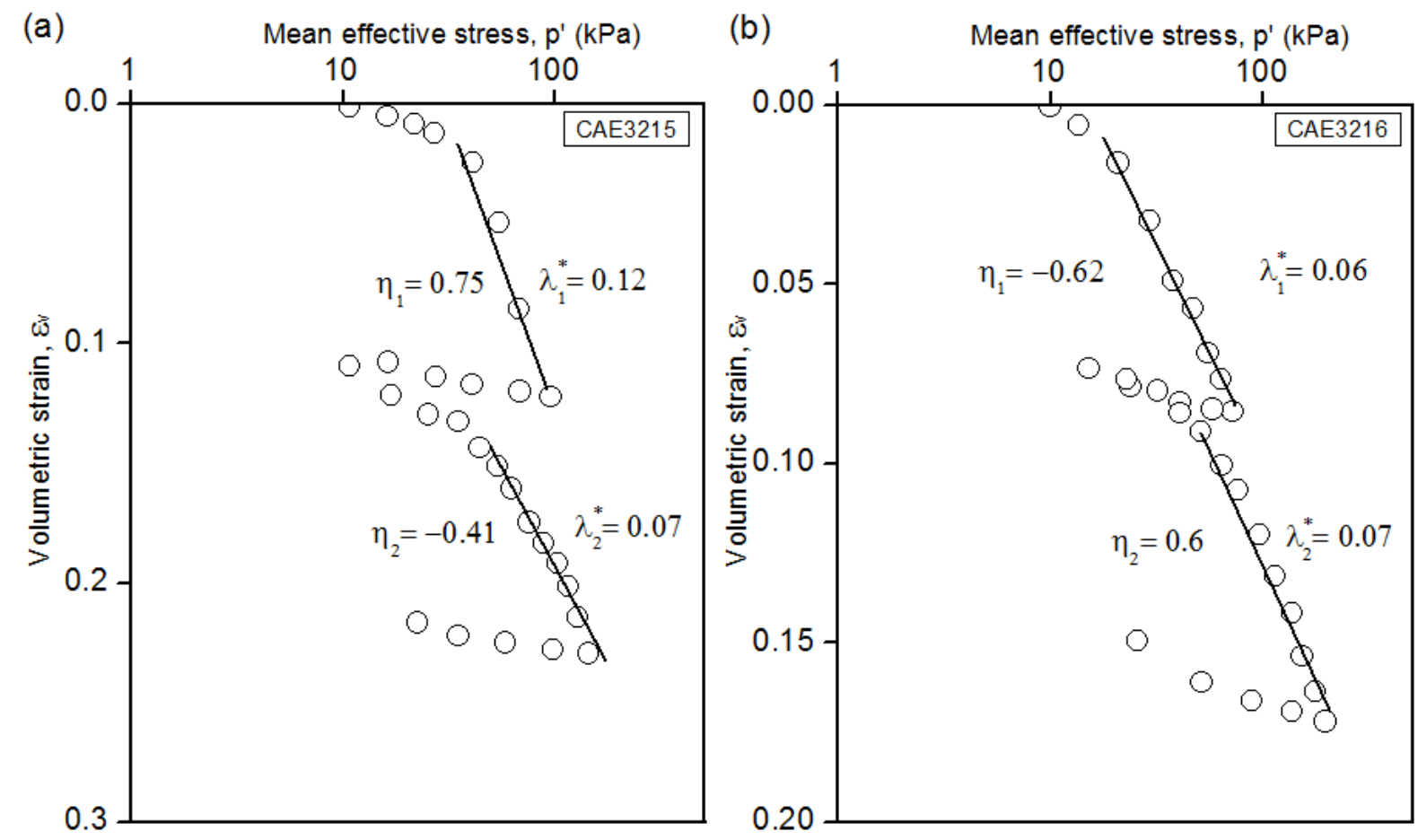

Figure 2.11 Results of IAC tests on remolded Murro clay (Karstunen and Koskinen 2008)

Test CAE3215 includes two loading stages. In the first stage, the sample was consolidated at a stress ratio of $\eta_{1}=0.75$, which yielded a modified compressive index $\lambda_{1}^{*}=0.12$ from the linear normal consolidation line. It is worth noted that the modified compressive index $\lambda^{*}=\frac{\lambda}{1+e_{0}}$ is used here for normalization purpose, given the variation of initial void ratios noticed from different tests. Similarly, the volumetric strain $\varepsilon_{v}=\frac{\Delta e}{1+e_{0}}$ is used instead of $\Delta e$. After the first loading stage, the sample was unloaded following the same stress ratio and then reloaded at $\eta_{2}=-0.41$, where the loading switched from compression to extension. A lower $\lambda_{2}^{*}=0.07$ has been yielded at this stage. It is understood that $\eta_{1}=0.75$ is of higher absolute value than $\eta_{2}=-0.41$, hence $\eta_{1}$ induces more significant $d \varepsilon_{d}^{p}$ than $\eta_{2}$ as per the plastic shearing volumetric change law of MCC model:

$$
\frac{d \varepsilon_{d}^{p}}{d \varepsilon_{v}^{p}}=\frac{2(\eta-\alpha)}{M^{2}-\eta^{2}}
$$


Test CAE3216 has two loading stages with very similar stress ratios in values $\left(\eta_{1}=-0.62, \eta_{2}=\right.$ $0.60)$. Two stages of loading results in rather close modified compressive index. The first loading stage yields an approximate $\lambda_{1}^{*}=0.06$, slightly lower than the second stage $\lambda_{2}^{*}=0.07$.

\subsubsection{EXPERIMENTAL RESULTS OF IAC TESTS ON REMOLDED OTANIEMI CLAY}

Two IAC tests, including CAE3519 and CAE3820 performed on the remolded Otaniemi clay (Karstunen and Koskinen 2008), demonstrate the behavior similar to remolded Murro clays, as shown in Figure 2.12. Test CAE3519 yields the same compression index $\left(\lambda_{1}^{*}=\lambda_{2}^{*}=0.07\right)$, from two distinct stress ratios, i.e., $\eta_{1}=-0.51$ and $\eta_{2}=0.52$. The results appear to confirm that the compressibility of soil is associated with stress ratios. Test CAE3820 demonstrates the same observation, see Figure 2.12 (b).

In summary, four IAC tests performed on two types of remolded soft clays in Finland suggest that soft clays manifest the dependence of compressibility on the applied stress ratios during IAC tests.
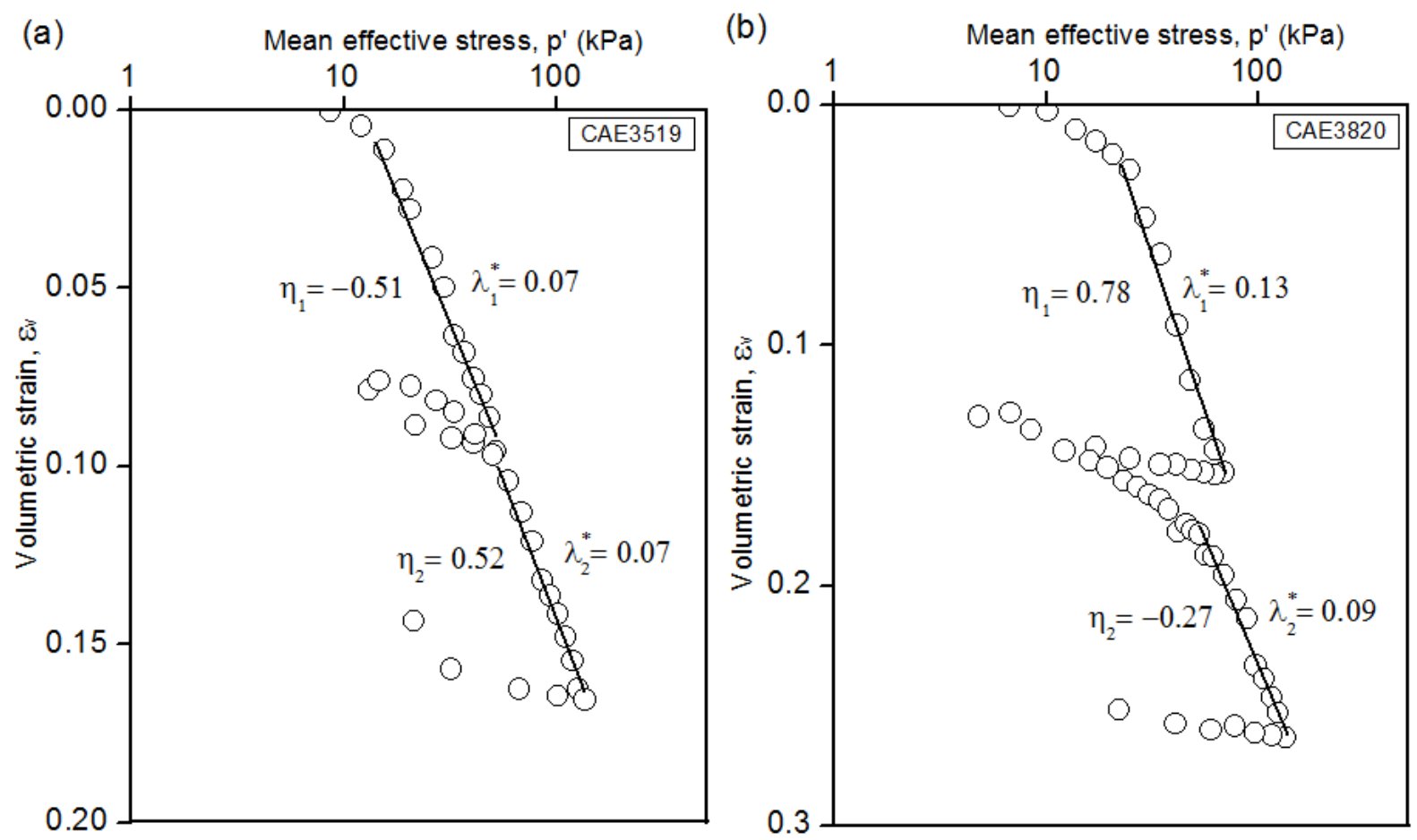

Figure 2.12 Results of IAC tests on remolded Otaniemi clays (Karstunen and Koskinen 2008) 


\subsubsection{EXPERIMENTAL RESULTS OF IAC TESTS ON OTANIEMI CLAY}

In this section, four anisotropic consolidation tests on intact Otaniemi clay (Wiltafsky et al. 2002, Wheeler et al. 2003) are presented in Figure 2.13 and Figure 2.14. The results further validate the phenomenon observed in remolded soft clays. It is also noticed that the intact Otaniemi clay, although sedimented in brackish water, doesn't exhibit apparent destructuration featured by Sshaped compression curves.
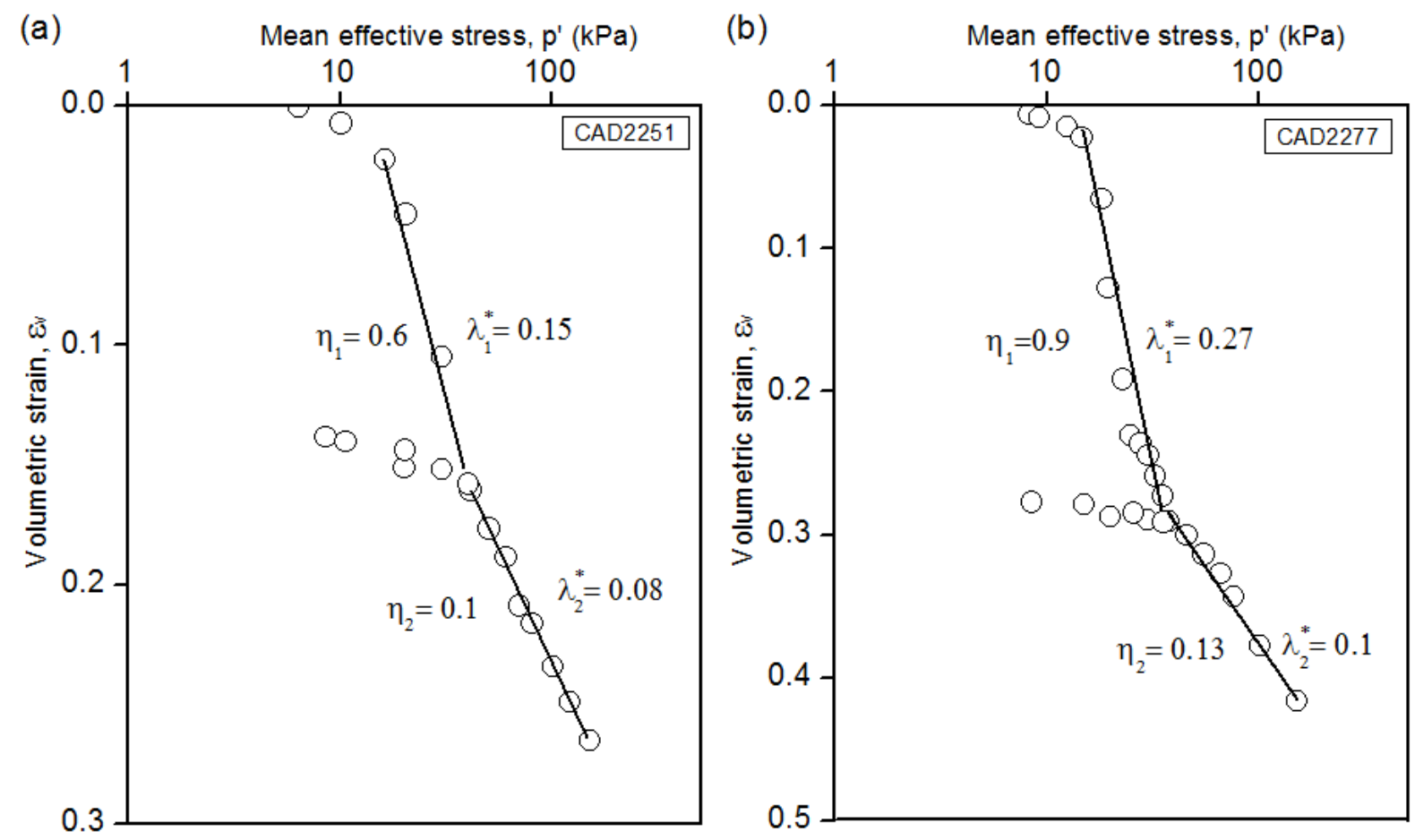

Figure 2.13 Results of IAC tests on undisturbed Otaniemi clay (Wheeler et al. 2003) 

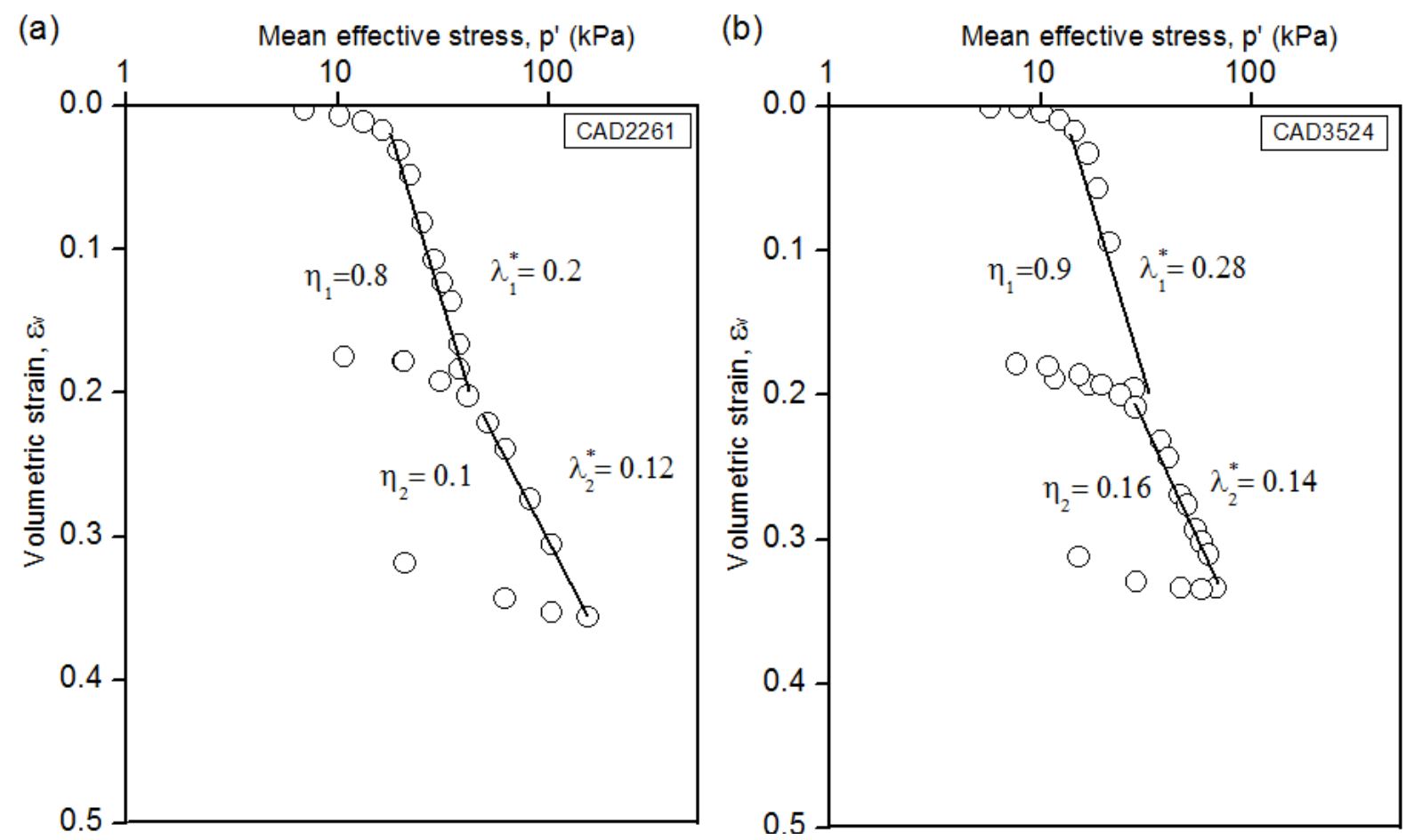

Figure 2.14 Results of IAC tests on undisturbed Otaniemi clay (Wiltafsky et al. 2002)

\subsubsection{SUMMARY OF IAC TEST RESULTS}

A total of 22 IAC tests have been summarized in Figure 2.15. The observed compression indices are interpreted as $\lambda_{1}^{*} / \lambda_{2}^{*}$, denoted the ratio between the compression index from two loading stages. In addition, the ratios are plotted against $\left|\eta_{1}\right|-\left|\eta_{2}\right|$, the corresponding stress ratio difference. This practice is to investigate how much the increase of $\eta$ would result in how much increase in $\lambda^{*}$ for each individual sample. 
(a)

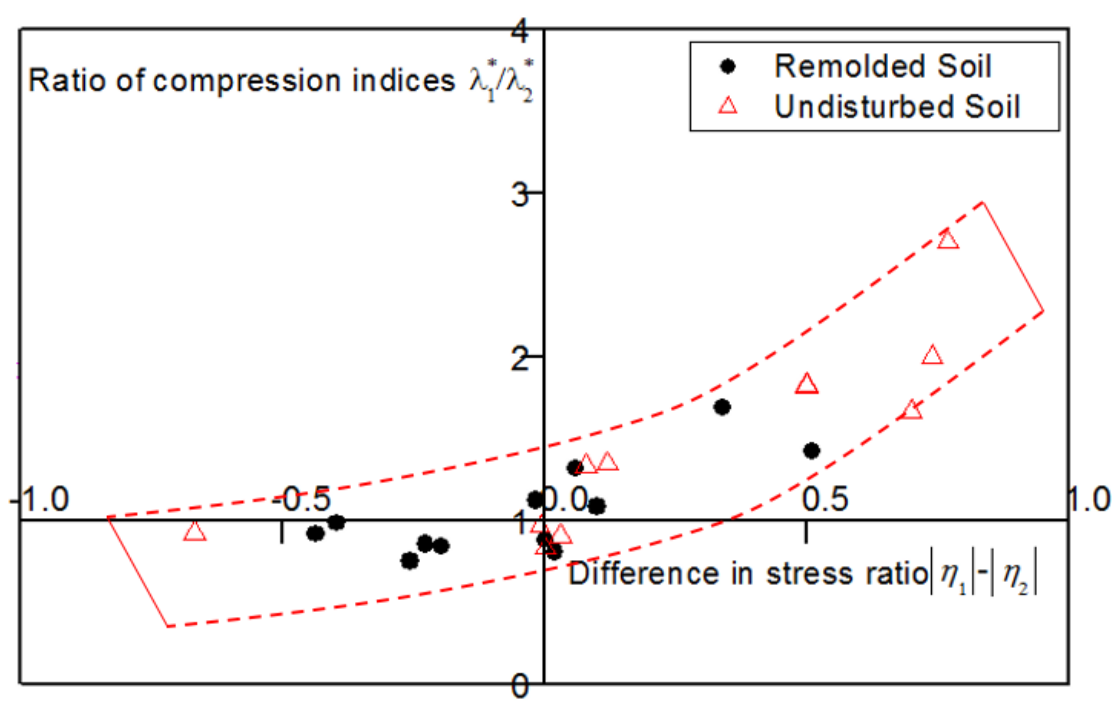

(b)

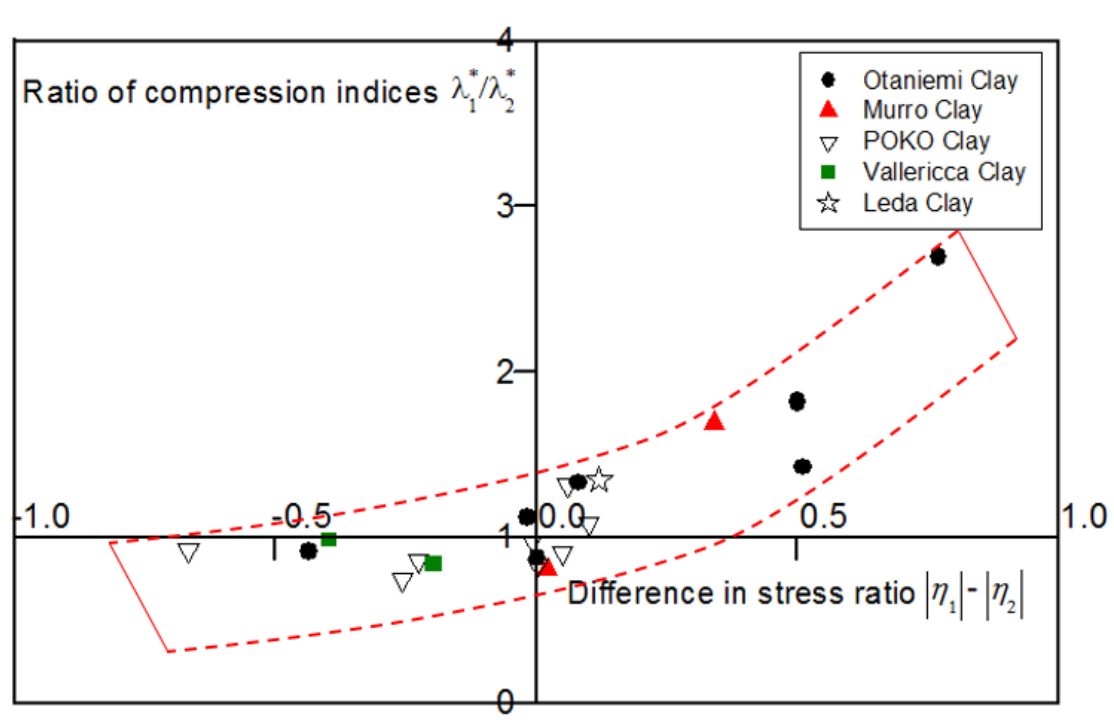

Figure 2.15 Summary of dependence of compression indices on stress ratios

The result summary shows explicitly the dependence of $\lambda^{*}$ on $\eta$. This behavior can be explained by yield surface softening owing to $d \varepsilon_{d}^{p}$. It means that $d \varepsilon_{d}^{p}$ could lead to negative $d p_{m}^{\prime}$, resulting in steeper compression curves, and thus higher $\lambda^{*}$.

One possible approach to simulate this behavior is to incorporate yield surface deviatoric softening into the volumetric hardening law. This will be detailed in the subsequent chapter. 
It is also of interests to plot all data points into the first quadrant. As such, $\eta_{1}-\eta_{2}$ has now been replaced by the normalized stress ratio difference $\frac{\left|\eta_{H}\right|-\left|\eta_{L}\right|}{M}$, where $\eta_{H}$ and $\eta_{L}$ represent the higher and the lower $\eta$ applied in the same test, likewise for $\lambda_{H}^{*}$ and $\lambda_{L}^{*}$. The results are plotted in Figure 2.16. It is found that exponential functions provide a better regression for the analyzed data. However, parabolic, quadratic, and cubic functions, are found to lead to significantly lower $R^{2}=$ $0.4 \sim 0.5$, compared to $R^{2}=0.79$ as obtained by an exponential function.

It should be noted that two data points located close to the origin are not considered in this analysis. Both tests do not employ obviously different stress ratios in their loading-reloading cycles, and thus can't signify the influence of stress ratios on soil compressibility. Moreover, there are three points located at $\mathrm{x}$ axis also considered as outliers. These three points are far from the rest of data points and are potentially explained by natural variation of the soil samples. Disregarding them in the regression analysis significantly improves the $R^{2}$ by 0.3 .

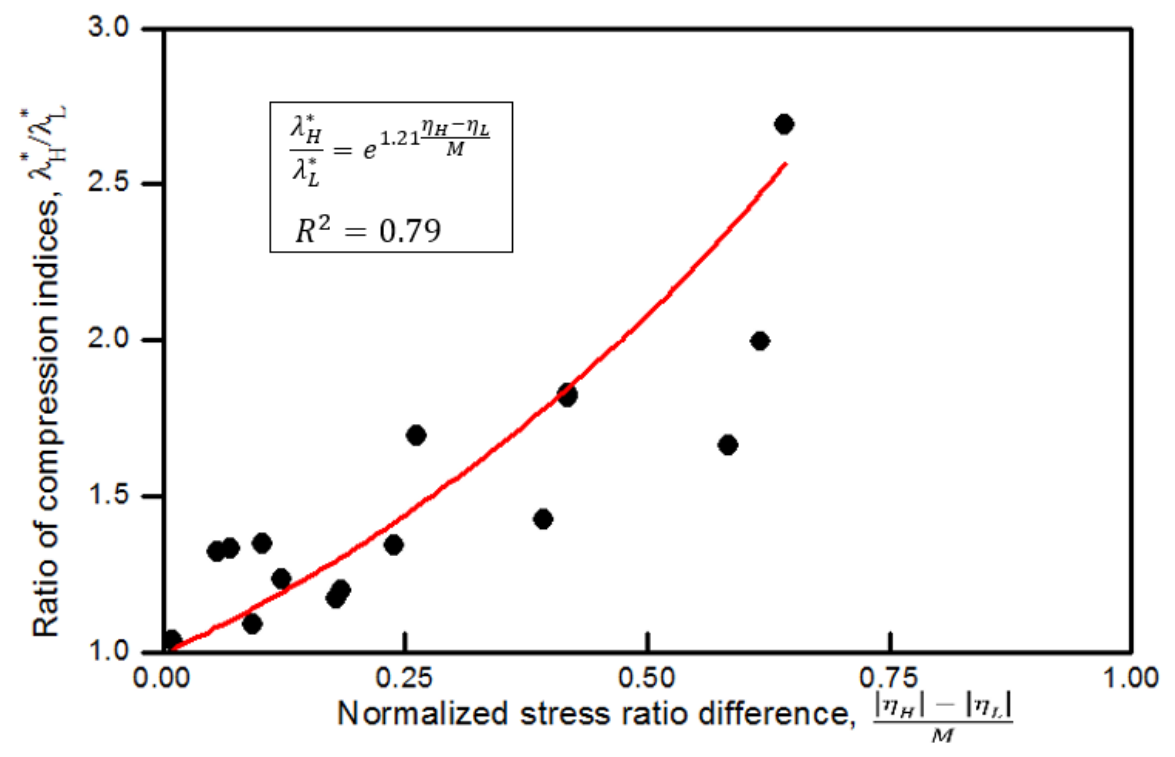

Figure 2.16 Summary of dependence of compression indices on normalized stress ratios

For a total of 15 tests studied in this research, an exponential dependence has been observed:

$$
\frac{\lambda_{H}^{*}}{\lambda_{L}^{*}}=e^{1.21 \frac{\eta_{H}-\eta_{L}}{M}}
$$


When assigning $\eta_{L}=0, \lambda_{L}^{*}$ reduces to the isotropic case where $\lambda_{L}^{*}=\lambda_{0}^{*}$. The following equation provides an empirical correlation to estimate the compression indices at any stress ratios lower than the critical state stress ratio $M$.

$$
\lambda_{H}^{*}=e^{1.21 \frac{\eta_{H}}{M}} \lambda_{0}^{*}
$$

When loaded at the critical stage $M$, [2.36] suggests the limit of compressibility increase by

$$
\lambda_{M}^{*}=e^{1.21} \lambda_{0}^{*} \approx 3.35 \lambda_{0}^{*}
$$

\subsection{SUMMARY AND CONCLUSIONS}

The scope of this chapter can be mainly summarized into two parts.

The first part reviews a number of hardening and softening soil models for soft clay and sand. These models can be generally classified into yield surface models and failure surface models. The

review shows that for failure surface models, it is a common practice to include both $d \varepsilon_{v}^{p}$ and $d \varepsilon_{d}^{p}$ influence in failure surface evolution. On the other hand, yield surface models tend to agree on the assumption that $d \varepsilon_{v}^{p}$ induces yield surface hardening, while the influence of $d \varepsilon_{d}^{p}$ is not agreed or often ignored. There are a limited number of theoretical models that assume $d \varepsilon_{d}^{p}$ contributes to hardening in soft clay, but this assumption has not been supported by experimental evidences.

The second part presents a number of published IAC tests performed on Finnish soft clays. The reanalysis of test results reveals the dependence of soil compressibility on stress ratios. The investigated clays appear to be more compressible when subject to higher stress ratios than those under low stress ratios. Consequently, the stress-ratio-induced $d \varepsilon_{d}^{p}$ could contribute to softening in soft clay.

Take the above-mentioned evidence into consideration, a modified deviatoric softening model is needed for soft clays. This new softening model is expected to improve compressibility prediction for soft clay subject to various stress ratios. 


\section{DEVELOPMENT OF A DEVIATORIC SOFTENING FOR SOFT CLAYS}

\subsection{INTRODUCTION}

In this chapter, a new yield surface deviatoric softening model is proposed for soft clays. This modified deviatoric softening law is based on the volumetric hardening law of MCC model. The softening law requires only one additional parameter, $\varsigma$, to govern the contribution of $d \varepsilon_{d}^{p}$ to yield surface softening.

Furthermore, the softening law is combined with other soft clay features, becoming a new model called MEVP-DS. These features contain destructuration, yield surface anisotropy, and viscosity. Then, MEVP-DS has been coded in FORTRAN, compiled into dynamic-link library, and implemented in Plaxis V8.2 software.

The verification of MEVP-DS model is performed through the simulation of a number of IAC tests on Finnish Otaniemi clay. The simulated tests were collected from published literatures and studied in Chapter 2. Destructuration and viscosity are considered in the simulation to be consistent with literatures.

Model predictions by MEVP-DS are compared with two reduced versions equivalent to the wellestablished anisotropic S-CLAY1 and isotropic MCC model. Model results show that MEVP-DS is able to improve the estimations of soil responses, i.e., volumetric strain, deviatoric strain. The model sensitivity to $\varsigma$ is also discussed at the end of this chapter.

\subsection{A MODIFIED DEVIATORIC SOFTENING LAW FOR SOFT CLAYS}

\subsubsection{A MODIFIED DEVIATORIC SOFTENING LAW}

Chapter 2 has demonstrated the dependence of soil compressibility on $\eta$ from a number of IAC tests on Finnish soft clays. The conclusion implies that the $\eta$ induced $d \varepsilon_{d}^{p}$ could contribute to the $d p_{m}^{\prime}$ evolution in soft clays. Recall that for analyzed tests, soil samples appear to exhibit higher $\lambda$ 
when subject to higher $\eta$ than those under lower $\eta$. To reproduce this behavior, one can incorporate $d \varepsilon_{d}^{p}$ into the existing volumetric hardening law.

In this dissertation, the author modifies the biased yield surface hardening law (Krenk 2000, Liu et al. 2013) to become the yield surface deviatoric softening law. The modification includes introducing $M$ and a weighting factor $\varsigma$ to the model.

$$
d p_{m 0}^{\prime}=\frac{v p_{m 0}^{\prime}}{\lambda-\kappa}\left(d \varepsilon_{v}^{p}-\varsigma \frac{\eta}{M} d \varepsilon_{d}^{p}\right)
$$

where $\quad p_{m 0}^{\prime}=$ size of intrinsic yield surface

$$
\begin{aligned}
& d p_{m 0}^{\prime}=\text { increment of intrinsic yield surface size } \\
& \varsigma=\text { a weighting factor that governs the deviatoric softening }
\end{aligned}
$$

The deviatoric softening law is introduced to the intrinsic yield surface. This attempt is to accommodate the structured yield surface associated with destructuration models. Test simulations using this modified deviatoric softening law show that values of $\varsigma$ calibrated for Finnish clay modeling range from 4 to 10 . When destructuration is considered, $\varsigma$ calibrated for sensitive Leda clay could be around 2 to 4 . It is because both deviatoric softening and destructuration consider yield surface softening. The difference is that deviatoric softening describes the intrinsic yield surface, while destructuration describes the structured yield surface.

In this softening law, $d \varepsilon_{d}^{p}$ will cause $p_{m 0}^{\prime}$ to shrink, leading to a steeper compression line in $\varepsilon_{\mathrm{v}} \sim \ln \mathrm{p}^{\prime}$ space. The weighting factor $\zeta$ is introduced to govern the contribution of deviatoric softening. Meanwhile, $d \varepsilon_{v}^{p}$ still contributes to yield surface hardening. The model can be reduced to the volumetric hardening law of MCC when either of $\varsigma$ or $\eta$ becomes zero.

Recall the plastic shearing volumetric change law of an inclined yield surface (Wheeler 1997):

$$
\frac{d \varepsilon_{d}^{p}}{d \varepsilon_{v}^{p}}=\frac{2(\eta-\alpha)}{M^{2}-\eta^{2}}
$$

The $d \varepsilon_{d}^{p}$ can be substituted by $d \varepsilon_{v}^{p}$ based on the plastic shearing volumetric change law. Combine Equation [3.1] and [3.2]: 


$$
d p_{m 0}^{\prime}=\frac{v p_{m 0}^{\prime}}{\lambda-\kappa} d \varepsilon_{v}^{p} \frac{M^{3}-(M+2 \varsigma) \eta^{2}+2 \alpha \varsigma \eta}{M\left(M^{2}-\eta^{2}\right)}
$$

Move $d \varepsilon_{v}^{p}$ to the left and do the integral:

$$
\varepsilon_{v}^{p}=\frac{\lambda-\kappa}{v} \ln p_{m 0}^{\prime} \frac{M\left(M^{2}-\eta^{2}\right)}{M^{3}-(2 \varsigma+M) \eta^{2}+2 \alpha \varsigma \eta}+\varepsilon_{v 0}^{p}
$$

where $\varepsilon_{v 0}^{p}$ is the initial plastic volumetric strain. Consequently, the $\varepsilon_{\mathrm{v}} \sim \ln \mathrm{p}^{\prime}$ relationship under general stress conditions would be:

$$
\varepsilon_{v}=\frac{\lambda}{v} \ln p_{m}^{\prime} \frac{M\left(M^{2}-\eta^{2}\right)}{M^{3}-(2 \varsigma+M) \eta^{2}+2 \alpha \varsigma \eta}+\varepsilon_{v 0}
$$

where $\varepsilon_{v 0}$ is the volumetric strain. The general stress and strain relationship within the anisotropic elasto-plasticity framework would be:

$$
\left[\begin{array}{l}
d \varepsilon_{v}^{p} \\
d \varepsilon_{q}^{p}
\end{array}\right]=\frac{\lambda-\kappa}{v p^{\prime}} \cdot \frac{M}{M^{3}-(2 \varsigma+M) \eta^{2}+2 \alpha \varsigma \eta} \cdot \frac{M^{2}-\eta^{2}}{M^{2}+\eta^{2}}\left[\begin{array}{cc}
M^{2}-\eta^{2} & 2 \eta \\
2 \eta & 4 \eta^{2}
\end{array}\right] \cdot\left[\begin{array}{c}
d p^{\prime} \\
d q
\end{array}\right]
$$

\subsubsection{FAMILY OF COMPRESSION LINES OF THE NEW SOFTENING LAW}

This section aims to investigate how compression lines are influenced by the new deviatoric softening law. Note that anisotropy has been ignored to assist with the description. Before discussing the new softening law, we recall the compression lines of MCC model in $\mathrm{v}-\ln \mathrm{p}^{\prime}$ space.

There are three IAC tests on a soft soil applied with $\eta=0, \eta$, and $\eta=M$, respectively. In the $p^{\prime}-$ $q$ space shown in Figure 3.1, three stress paths intersect a family of MCC yield loci. Yield surfaces with increased sizes represent hardening.

For MCC model, three stress ratios will result in three parallel compression lines in $v-\ln \mathrm{p}^{\prime}$ space as specified in Figure 3.2, where the isotropic normal consolidation line (iso-ncl) is positioned on the top. $N$ and $\Gamma$ are initial void ratios of the isotropic line and the critical state line (csl) starting at $p^{\prime}=1 \mathrm{kPa} . v_{\lambda}$ is the initial specific volume if the soil is loaded at an arbitrary stress ratio. Each yield locus is associated with infinite numbers of unloading-reloading lines ( $u r l)$. For the iso-ncl line, the calculation of specific volumes follows 


$$
v=N-\lambda \ln p_{m}^{\prime}
$$

If the sample is unloaded from $p_{m}^{\prime}$ to $p^{\prime}$, the specific volume at stress state $p^{\prime}$ is determined by

$$
v_{p^{\prime}}=N-\lambda \ln p_{m}^{\prime}+\kappa \ln \frac{p_{m}^{\prime}}{p^{\prime}}
$$

Note that $v_{p}$, can be also achieved from $\eta=\eta$ line with the initial volume of $v_{\lambda}$

$$
v_{p^{\prime}}=v_{\lambda}-\lambda \ln p^{\prime}
$$

Combing [3.8] and [3.9] gives

$$
v_{\lambda}=N-(\lambda-\kappa) \ln \frac{p_{m}^{\prime}}{p^{\prime}}
$$

Recall that from MCC yield locus has the following relation:

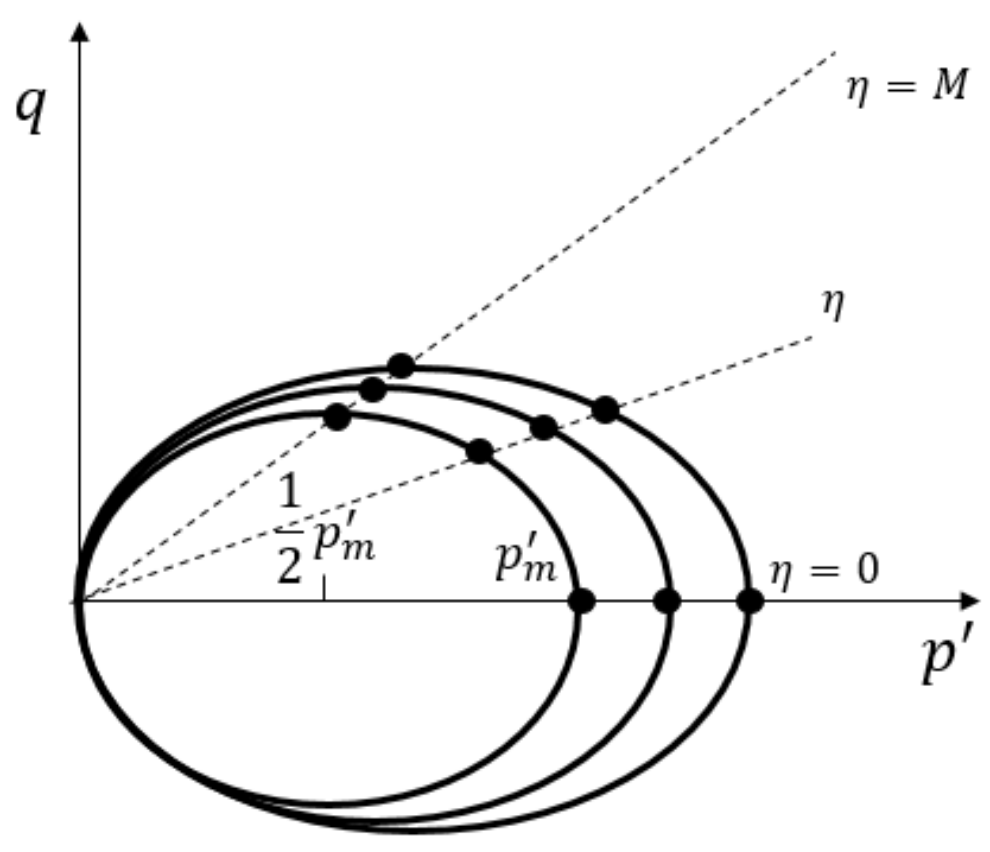

Figure 3.1 Critical state line and intersection of Modified Cam-Clay yield surface with different stress ratios (Wood 1994) 


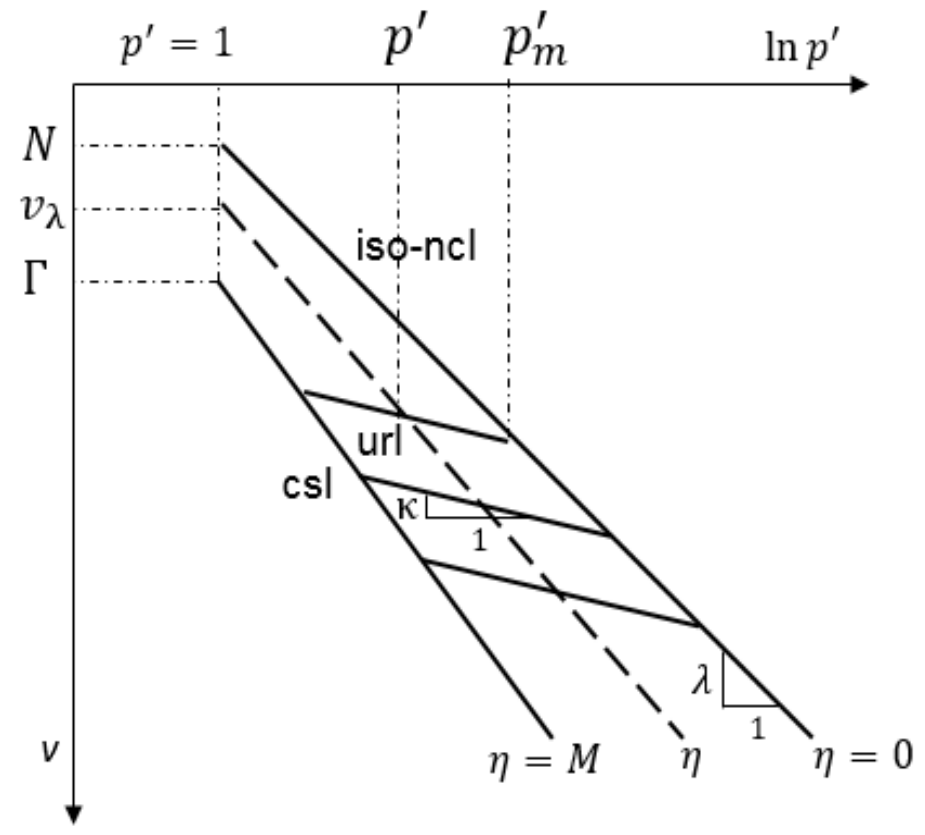

Figure 3.2 Family of compression lines in $v-\ln p^{\prime}$ space (Wood 1994)

$$
\frac{p_{m}^{\prime}}{p^{\prime}}=\frac{M^{2}+\eta^{2}}{M^{2}}
$$

Thus, $v_{\lambda}$ resulted from any arbitrary stress ratios is determined

$$
v_{\lambda}=N-(\lambda-\kappa) \ln \frac{M^{2}+\eta^{2}}{M^{2}}
$$

and hence, at the critical state $\eta=M$

$$
\Gamma=N-(\lambda-\kappa) \ln 2
$$

However, when the new softening law is introduced, compression lines from various stress ratios will not be parallel anymore. As proposed by the deviatoric softening law, any stress paths of stress ratios $\eta>0$ will result in compression lines steeper than the iso-ncl line According to [3.5], by ignoring the inclination $(\alpha=0)$, the specific volume at the stress state $p^{\prime}$ following the $\eta$ line starting from $v_{\lambda}$ is 


$$
v_{p^{\prime}}=v_{\lambda}-\lambda \frac{M\left(M^{2}-\eta^{2}\right)}{M^{3}-(2 \varsigma+M) \eta^{2}} \ln p^{\prime}
$$

Combine [3.18] with [3.8], the determination of $v_{\lambda}$ follows

$$
\begin{gathered}
v_{\lambda}=N+\lambda \frac{M\left(M^{2}-\eta^{2}\right)}{M^{3}-(2 \varsigma+M) \eta^{2}} \ln p^{\prime}-\lambda \ln p_{m}^{\prime}+\kappa \ln \frac{p_{m}^{\prime}}{p^{\prime}} \\
v_{\lambda}=N+\lambda \frac{M\left(M^{2}-\eta^{2}\right)}{M^{3}-(2 \varsigma+M) \eta^{2}} \ln p^{\prime}-\lambda \ln p_{m}^{\prime}+\lambda \ln p^{\prime}+\kappa \ln \frac{p_{m}^{\prime}}{p^{\prime}}-\lambda \ln p^{\prime} \\
v_{\lambda}=N-(\lambda-\kappa) \ln \frac{p_{m}^{\prime}}{p^{\prime}}+\lambda\left(\frac{M\left(M^{2}-\eta^{2}\right)}{M^{3}-(2 \varsigma+M) \eta^{2}}-1\right) \ln p^{\prime}
\end{gathered}
$$

[3.17] provides the determination of $v_{\lambda}$ at any arbitrary stress state. Replace $\frac{p_{m}^{\prime}}{p^{\prime}}$ with $\frac{M^{2}+\eta^{2}}{M^{2}}$ as we did in [3.11], [3.17] becomes

$$
v_{\lambda}=N-(\lambda-\kappa) \ln \frac{M^{2}+\eta^{2}}{M^{2}}+\lambda\left(\frac{M\left(M^{2}-\eta^{2}\right)}{M^{3}-(2 \varsigma+M) \eta^{2}}-1\right) \ln p^{\prime}
$$

Note that for an initial condition $p^{\prime}=p_{m}^{\prime}=1, \Gamma$ is determined as

$$
\Gamma=N-(\lambda-\kappa) \ln 2
$$

Therefore, the modified softening law will result in the same gap between iso-ncl and $c s l$ at the initial stage where $p^{\prime}=1$. However, the gap increases with respect to $p^{\prime}$, which is determined by

$$
v_{i s o-n c l}-v_{c s}=(\lambda-\kappa) \ln 2+\lambda\left(\frac{M\left(M^{2}-\eta^{2}\right)}{M^{3}-(2 \varsigma+M) \eta^{2}}-1\right) \ln p^{\prime}
$$

It could be noticed that [3.20] will lead to infinity when $\eta$ approaches $M$. In reality, as suggested in Chapter 2, the critical state line approaches the limit of $3.35 \lambda$.

\subsection{MEVP-DS MODEL}

In this section the modified deviatoric softening law is incorporated into the existing MEVP model (Feng et al. 2014, Liu et al. 2017, Shi and Salloum 2018). The model is called MEVP-DS model (modified MEVP model with deviatoric softening). 
MEVP model combines Yin's EVP framework, and yield surface anisotropy and destructuration of the well-established S-CLAY1S model (Koskinen et al. 2002). The original FORTRAN codes of MEVP model are provided by Dr. Jian-Hua Yin from The Hong Kong Polytechnic University.

\subsubsection{ELASTICITY}

The calculation of elastic volumetric strain increment $d \varepsilon_{v}^{e}$ and elastic deviatoric strain increment $d \varepsilon_{d}^{e}$ of MEVP-DS follows the same way as Modified Cam-Clay:

$$
\left[\begin{array}{l}
d \varepsilon_{v}^{e} \\
d \varepsilon_{d}^{e}
\end{array}\right]=\left[\begin{array}{cc}
\frac{\kappa}{v p^{\prime}} & 0 \\
0 & \frac{1}{3 G}
\end{array}\right]\left[\begin{array}{l}
d p^{\prime} \\
d q
\end{array}\right]
$$

where $G$ is the shear modulus determined as

$$
G=\frac{3(1-2 \mu) v p^{\prime}}{2(1+\mu) \kappa} \sqrt{E_{\text {ratio }}}
$$

where $\mu$ is the poisson ratio, $E_{\text {ratio }}=\frac{E_{h}}{E_{v}}$ the ratio between horizontal and vertical Young's modulus. $E$ is defined below,

$$
E=\frac{3(1-2 \mu) v p^{\prime}}{\kappa} E_{\text {para }}
$$

where $E_{\text {para }}$ is a constant specifying anisotropic elasticity. $E_{\text {para }}$ is taken as 1 for isotropic elasticity.

\subsubsection{EVOLUTIONAL ANISOTROPY OF YIELD SURFACE}

MEVP-DS model employs an inclined intrinsic yield surface

$$
f=g=\left(q-\alpha p^{\prime}\right)^{2}-\left(M^{2}-\alpha^{2}\right)\left(p_{m 0}^{\prime}-p^{\prime}\right) p^{\prime}=0
$$

Assuming an associated flow, the yield surface rotation is governed by $\alpha$ and size controlled by $p_{m 0}^{\prime}$, see Figure 3.3 . 


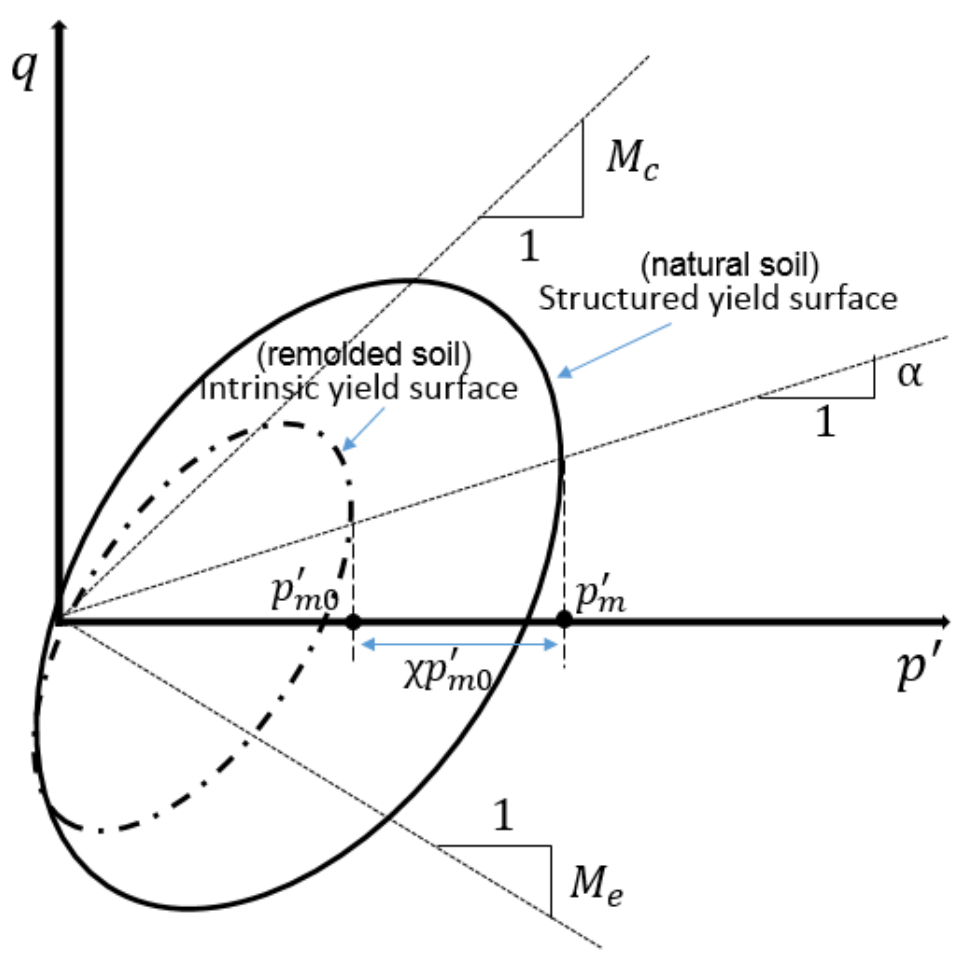

Figure 3.3 Inclined yield surfaces of MEVP-DS model (Yin and Karstunen 2008)

Structure bonding is assumed to grant additional yield surface size to undisturbed clays compared to remoulded clays. The initial bonding is described using a scalar state variable $\chi_{0}$, see Figure 3.3. $(1+\chi)$ describes the initial ratio of $p_{m 0}^{\prime}$ and $p_{m}^{\prime}$ obtained from undisturbed and remoulded samples respectively.

$$
p_{m}^{\prime}=(1+\chi) p_{m 0}^{\prime}
$$

It is assumed that the new deviatoric softening law affects only the size of intrinsic yield surface $p_{m 0}^{\prime}$. The evolution of $p_{m 0}^{\prime}$ is assumed to be associated with both $d \varepsilon_{v}^{v p}$ and $d \varepsilon_{d}^{v p}$, to comply with the Yin's EVP framework. The process is governed by both $\varsigma$ and $\eta$, proposed in Section 3.2.1

$$
d p_{m 0}^{\prime}=\frac{v p_{m 0}^{\prime}}{\lambda-\kappa}\left(d \varepsilon_{v}^{v p}-\varsigma \frac{\eta}{M} d \varepsilon_{d}^{v p}\right)
$$

Assigning zero to $\varsigma$ will reduce the softening law to MCC volumetric hardening law

$$
d p_{m 0}^{\prime}=\frac{v p_{m 0}^{\prime}}{\lambda-\kappa} d \varepsilon_{v}^{p}
$$


Inclination $\alpha$ dynamically approaches $\frac{3 \eta}{4}$ and $\frac{\eta}{3}$ simultaneously during plastic straining, also controlled by both plastic volumetric strain $d \varepsilon_{v}^{v p}$ and plastic deviatoric strain $d \varepsilon_{d}^{v p}$.

$$
d \alpha=\omega\left[\left(\frac{3 \eta}{4}-\alpha\right)\left|d \varepsilon_{v}^{v p}\right|+\omega_{d}\left(\frac{\eta}{3}-\alpha\right) d \varepsilon_{d}^{v p}\right]
$$

The evolutional yield surface rotation is controlled by the absolute rate of yield surface rotation $\omega$ and the relative rate of yield surface rotation $\omega_{d}$.

\subsubsection{VISCO-PLASTICITY OF TIME-DEPENDENCE BEHAVIOR}

The EVP frame work employed by MEVP-DS stemmed from the equivalent time concept proposed by Yin and Graham (1994). In this model, the volumetric strain is taken as the sum of two components in isotropic stressing:

$$
\varepsilon_{v}=\varepsilon_{v}^{e p}+\varepsilon_{v}^{v p}=\varepsilon_{v o}^{e p}+\frac{\lambda}{v} \ln \left(\frac{p_{m d}^{\prime}}{p_{m o}^{\prime}}\right)+\frac{\psi}{v} \ln \left(\frac{t_{0}+t_{e}}{t_{0}}\right)
$$

where $\varepsilon_{v}^{e p}$ is the time-independent elasto-plastic volumetric strain, $\varepsilon_{v}^{v p}$ is the time-dependent visco-plastic volumetric strain, $\varepsilon_{v o}^{e p}$ is the initial time-independent elasto-plastic volumetric strain, $\lambda=\frac{\Delta e}{\Delta \ln p^{\prime}}$ is the slope of the normal consolidation line, $\psi=\frac{\Delta e}{\Delta \operatorname{lnt}}$ is the creep index, $t_{0}$ is the reference time that specifies when creep commences. $p_{m d}^{\prime}$ is the size of the dynamic yield surface. $t_{e}$ is the equivalent time defined as the time needed to creep from $t_{0}$ to the value of $\varepsilon_{v}$ under the constant $p_{m d}^{\prime} . t_{0}$ is usually taken as one day to avoid infinite strain and strain rate.

From Equation [3.29] the equivalent time $t_{e}$ can be calculated:

$$
t_{e}=-t_{0}+t_{0} \exp \left[\left(\varepsilon_{v}-\varepsilon_{v o}^{e p}\right) \frac{v}{\psi}\right]\left(\frac{p_{m d}^{\prime}}{p_{m}^{\prime}}\right)^{-\frac{\lambda}{\psi}}
$$

The derivative form of Equation [3.29] is:

$$
d \varepsilon_{v}=d \varepsilon_{v}^{e p}+d \varepsilon_{v}^{v p}=\frac{\kappa}{v p_{m d}^{\prime}} d p_{m d}^{\prime}+\frac{\psi}{v} \frac{1}{t_{0}+t_{e}} d t
$$

$\dot{\varepsilon}_{v}$ can be calculated using the expression of the equivalent time $t_{e}$ in Equation [3.31]: 


$$
\dot{\varepsilon}_{v}=\dot{\varepsilon}_{v}^{e p}+\dot{\varepsilon}_{v}^{v p}=\frac{\kappa}{v p_{m o}^{\prime}} \frac{d p_{m o}^{\prime}}{d t}+\frac{\psi}{v t_{0}} \exp \left[-\left(\varepsilon_{v}-\varepsilon_{v o}^{e p}\right) \frac{v}{\psi}\right]\left(\frac{p_{m d}^{\prime}}{p_{m o}^{\prime}}\right)^{\frac{\lambda}{\psi}}
$$

The tensor of viscoplastic strain rate $\dot{\varepsilon}_{i j}^{v p}$ is calculated according to the overstress framework in (Perzyna 1963, 1966).

$$
\dot{\varepsilon}_{i j}^{v p}=\gamma\langle\varphi(F)\rangle \frac{\partial g}{\partial \sigma_{i j}^{\prime}}=S \frac{\partial g}{\partial \sigma_{i j}^{\prime}}
$$

where $\gamma$ denotes the fluidity parameter and the scalar function $\varphi(F)$ is the flow function. $\frac{\partial g}{\partial \sigma_{i j}^{\prime}}$ determines the vector of plastic flow.

In Yin's EVP framework, $S$ is a scalar function replacing $\gamma\langle\varphi(F)\rangle$. The term $\langle\varphi(F)\rangle$ implies:

$$
\langle\varphi(F)\rangle= \begin{cases}\varphi(F) & \text { for } F>0 \\ 0 & \text { for } F \leq 0\end{cases}
$$

Based on the associate flow:

$$
f=g=\left(q-\alpha p^{\prime}\right)^{2}-\left(M^{2}-\alpha^{2}\right)\left(p_{m d}^{\prime}-p^{\prime}\right) p^{\prime}=0
$$

To interpolate $M$ between $M_{c}$ and $M_{e}, M$ is modified with Lode angle $\theta$ (Sheng at al. 2000)

$$
\begin{gathered}
M=M_{c}\left[\frac{2 d^{4}}{1+d^{4}+\left(1-d^{4}\right) \sin 3 \theta}\right]^{1 / 4} \\
\theta=\frac{1}{3} \sin ^{-1} \frac{-3 \sqrt{3} \bar{J}_{3}}{2 \bar{J}_{2}^{3 / 2}}
\end{gathered}
$$

where $d=\frac{3-\sin \varphi_{c}}{3+\sin \varphi_{c}}, \varphi_{c}$ is the friction angle based on Mohr-Coulomb criterion. $\theta$ is in the range from $-\frac{\pi}{6}$ to $\frac{\pi}{6}, \bar{J}_{2}=\frac{1}{2} s_{i j}: s_{i j}, \overline{J_{3}}=\frac{1}{3} s_{i j} s_{j k} s_{k l}$. $s_{i j}=\sigma_{i j}^{\prime}-p^{\prime} \delta_{i j}^{\prime}$ is the deviatoric stress tensor. $\delta_{i j}^{\prime}=1$ when $i=j$ and $\delta_{i j}^{\prime}=0$ when $i \neq j$.

From the plastic potential in [3.35] we have: 


$$
\left\{\begin{array}{l}
\dot{\varepsilon}_{v}^{v p}=S \frac{\partial f}{\partial p^{\prime}}=S\left(2 p^{\prime}-p_{m d}^{\prime}\right)\left(M^{2}-\alpha^{2}\right) \\
\dot{\varepsilon}_{d}^{v p}=S \frac{\partial f}{\partial q}=2 S\left(q-\alpha p^{\prime}\right)
\end{array}\right.
$$

Note that here we assume $\dot{\varepsilon}_{v}^{v p}$ to be a constant on the plastic potential. In other words, the $\dot{\varepsilon}_{v}^{v p}$ of the isotropic case in [3.32] is equal to the $\dot{\varepsilon}_{v}^{v p}$ (Yin and Graham 1999) in [3.38]. Combine [3.32] with [3.38], $S$ can be thus determined:

$$
S=\frac{\psi}{t_{0} v} \exp \left[-\left(\varepsilon_{v}-\varepsilon_{v o}^{e p}\right) \frac{v}{\psi}\right]\left(\frac{p_{m d}^{\prime}}{p_{m o}^{\prime}}\right)^{\frac{\lambda}{\psi}} \frac{1}{\left(2 p^{\prime}-p_{m d}^{\prime}\right)\left(M^{2}-\alpha^{2}\right)}
$$

\subsubsection{DESTRUCTURATION OF STRUCTURED YIELD SURFACE}

During the plastic straining, bonding $\chi$ will decrease as the result of $d \varepsilon_{v}^{v p}$ and $d \varepsilon_{d}^{v p}$. The rate of structuration is governed by two parameters: $\xi$, the absolute effectiveness of destructuration hardening and $\xi_{d}$, the relative effectiveness of destructuration hardening. The destructuration law follows Koskinen's model already explained in Figure 2.6.

$$
d \chi=-\xi \cdot \chi \cdot\left(\left|d \varepsilon_{v}^{v p}\right|+\xi_{d} \cdot d \varepsilon_{d}^{v p}\right)
$$

\subsubsection{IMPLEMENTATION OF MEVP-DS IN PLAXIS V8.2}

Both MEVP-DS and MEVP have been implemented in Plaxis V8.2 program as a user-defined model. The model was first programmed in FORTRAN language. After that, five subroutines associated with defined models were compiled into DLL (Dynamic Link Library) files. The DLL files can be then imported by Plaxis V8.2 for numerical simulations. Codes of MEVP-DS model are attached in Appendix A and Appendix B. Appendix A provides the subroutine "EVP.for" and Appendix B provides another subroutine "MY_CLAY.for". Note that "MY_CLAY.for" is the outline subroutine of MEVP-DS model. "EVP.for" is the most important subroutine containing constitutive models, which can be called in "MY_CLAY.for".

This section briefly introduces the structure of model codes and compilation details. For more information, readers can refer to the material manual (Plaxis 2002). 
Subroutine FORTRAN codes should be written following the task orders from IDTask 1 to IDTask 6 each executing a specfic part of the user-defined model, shown in Table 3.1.

Table 3.1 Main tasks to be defined in a Plaxis subroutine for user-defined model

\begin{tabular}{ll}
\hline Task No. & Contents \\
\hline ID Task=1 & Initialize state variables \\
ID Task $=2$ & Calculate stresses \\
ID Task $=3$ & Calculate material stiffness matrix \\
ID Task $=4$ & Return number of state variables \\
ID Task $=5$ & Inquire matrix properties \\
ID Task $=6$ & Calculate elastic material stiffness matrix \\
\hline
\end{tabular}

ID Task=2 is the most important task where "MY_CLAY.for" and "EVP.for" are called. In addition, other three subroutines are also called when running the subroutines. They are listed in Table 3.2.

Table 3.2 Other subroutines that need to be called by the main subroutine

\begin{tabular}{cc}
\hline Task No. & Contents \\
\hline USRMOD.FOR & Enable switch between different models. \\
USR_ADD.FOR & Store information such as names, parameters and state variables \\
HANDYLIB.FOR & A library that contains useful subroutines provided by Plaxis. \\
\hline
\end{tabular}

All five subroutines to be executed must be compiled into one DLL before implementation. Two types of FORTRAN compilers are supported by Plaxis including the Digital Visual FORTRAN Compiler and the Lahey FORTRAN compiler. One should ensure, however, that the version of the selected FORTRAN compiler should be earlier than the version of Plaxis software. Unsuccessful implementation will occur when Plaxis fails to recognize the DLL file compiled by a later version 
of the FORTRAN compiler. It is recommended using Lahey 95 FORTRAN compiler to be compatible to Plaxis V8.2 or more recent versions.

\subsection{MODEL VERIFICATION}

\subsubsection{MODEL DESCRIPTION}

In this section, MEVP-DS model is verified through the simulation of a number of IAC tests on Finnish clays. The purpose of verification is to demonstrate the improvement of MEVP-DS prediction if deviatoric softening is considered. The summary of simulated tests can be found in Table 3.3. The geometry of the model follows the sample dimension as the publication (Koskinen 2014). As seen in Figure 3.4, an axisymmetric model has been used in the analysis with the radius of $2.5 \mathrm{~cm}$ and the height of $10.0 \mathrm{~cm}$.

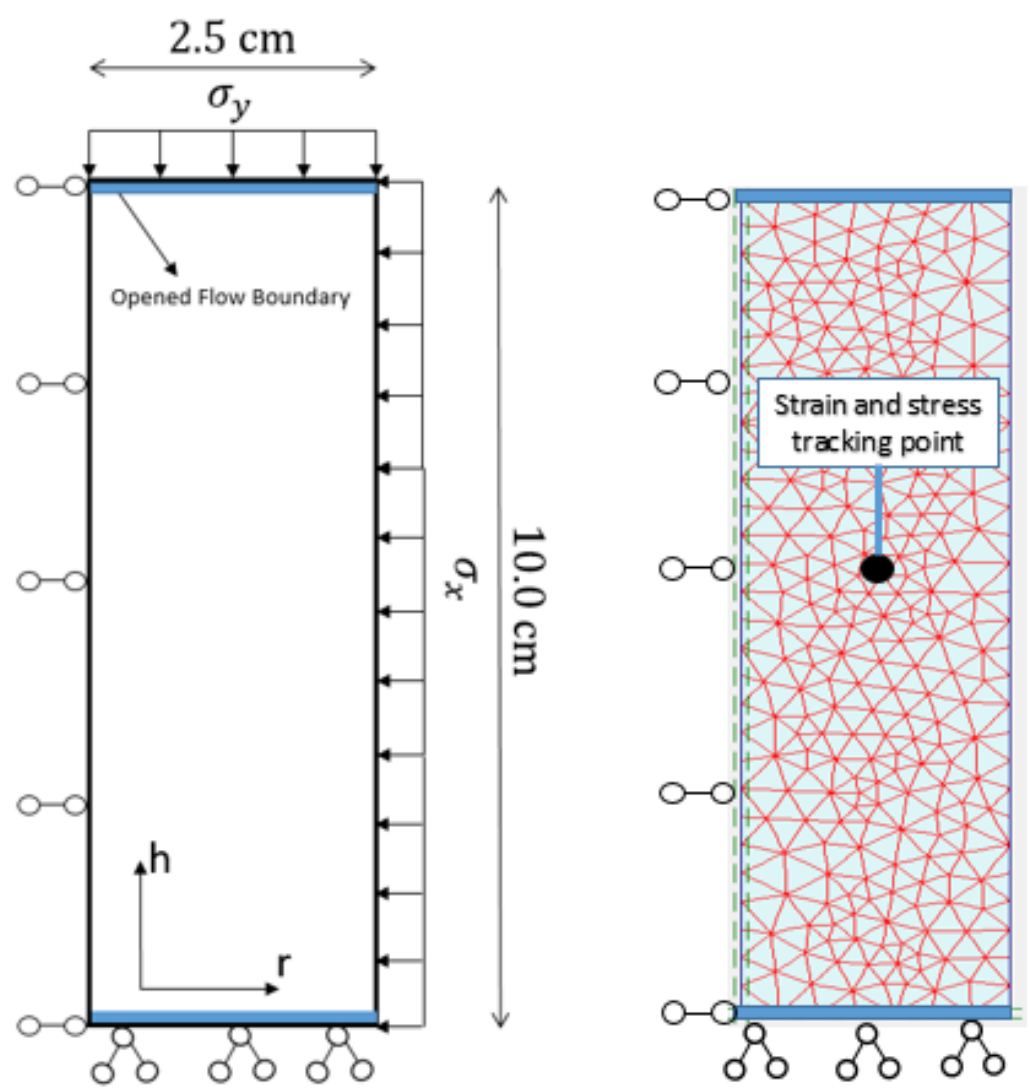

Figure 3.4 Axisymmetric sample geometry for test IAC modeling 
Table 3.3 Summary for the simulated tests

\begin{tabular}{ccc}
\hline Layer & $\boldsymbol{\eta}_{\mathbf{1}}$ & $\boldsymbol{\eta}_{\mathbf{2}}$ \\
& - & - \\
\hline CAD2251 & 0.60 & 0.10 \\
CAD2261 & 0.80 & 0.10 \\
CAD2277 & 0.90 & 0.13 \\
CAD3524 & 0.90 & 0.16 \\
CAE2544 & -0.59 & 0.51 \\
CAE3215 & 0.75 & -0.41 \\
CAE3820 & 0.78 & -0.27 \\
\hline
\end{tabular}

Horizontal displacements of the model are fixed at the left boundary, while both horizontal and vertical displacements are fixed at the bottom boundary. Drainage is allowed at both top and bottom boundaries. The geometry is disretized into a typical number of 208 axisymmetric triagular elements. This number of elements or more has been found to yield consistant model predictions. Loadings perpendicular to the top and right boundaries are applied. To simulate the same stress ratios as the published tests, following equations are used to convert $\eta, p^{\prime}$, and $q$, to the horizontal pressure $\sigma_{x}$ and the veritical pressure $\sigma_{y}$.

$$
\begin{aligned}
& \eta=\frac{3(1-K)}{(2 K+1)} \quad K=\frac{3-\eta}{2 \eta+3} \\
& \sigma_{y}=\left(\frac{3}{1+2 K}\right) p^{\prime} \quad \sigma_{x}=K \sigma_{y}
\end{aligned}
$$

Where the stress coefficient $K=\frac{\sigma_{x}}{\sigma_{y}}$ has been used to determine $\sigma_{x}$ and $\sigma_{y}$, which are applied to sample surfaces.

\subsubsection{PARAMETERS INPUT OF MEVP-DS MODEL}

Along with MEVP-DS model, two simplified versions of MEVP-DS are also used in the model simulation, see Figure 3.4. S-CLAY1 is the reduced version of MEVP-DS model by ignoring destructuration and creep. As such, S-CLAY1 is only able to consider anisotropy. In addition, by further ignoring yield surface anisotropy, S-CLAY1 is reduced to MCC. Not that destructuration and creep are not considered in MEVP-DS model for the analysis. The reason is to be consistent 
with references where the modeled tests are published (Zentar et al. 2002, Wheeler et al. 2003, Karstunen and Koskinen 2008, Koskinen 2014). In these literatures, tests were already modeled with S-CLAY1 and MCC. Check Table 3.5 and Table 3.6 for more details.

Table 3.4 Soil models used for IAC tests modeling

\begin{tabular}{cc}
\hline Case Name & Details \\
\hline MEVP-DS & Consider both deviatoric softening and anisotropy \\
S-CLAY1 & Reduced version of MEVP-DS. Able to consider anisotropy \\
MCC & Isotropic Modified Cam-Clay \\
\hline
\end{tabular}


Table 3.5 Values for the initial state parameters used in the test modeling (collected from publications)

\begin{tabular}{|c|c|c|c|c|c|c|c|c|c|c|c|c|c|c|c|c|c|}
\hline Test No & Soil type & $\begin{array}{c}\gamma \\
\mathrm{kN} / \mathrm{m}^{3}\end{array}$ & $\begin{array}{l}\boldsymbol{p}_{\boldsymbol{m}}^{\prime} \\
\mathrm{kPa}\end{array}$ & $\begin{array}{c}\boldsymbol{k}_{\boldsymbol{0}} \\
\times 10^{-4} \mathrm{~m} / \mathrm{d} \\
\end{array}$ & $\begin{array}{c}K_{0} \\
- \\
\end{array}$ & $\begin{array}{l}v \\
-\end{array}$ & $\begin{array}{c}e_{0} \\
- \\
\end{array}$ & $\begin{array}{l}\psi \\
- \\
\end{array}$ & $\begin{array}{c}\boldsymbol{t}_{\mathbf{0}} \\
\text { day }\end{array}$ & $\begin{array}{c}\alpha_{0} \\
- \\
\end{array}$ & $\begin{array}{l}\omega \\
- \\
\end{array}$ & $\begin{array}{c}\omega_{d} \\
- \\
\end{array}$ & $\begin{array}{c}\chi_{0} \\
- \\
\end{array}$ & $\begin{array}{l}\xi \\
-\end{array}$ & $\begin{array}{l}\xi_{\mathrm{d}} \\
- \\
\end{array}$ & $\begin{array}{c}M_{c} \\
- \\
\end{array}$ & $\begin{array}{c}M_{e} / M_{c} \\
- \\
\end{array}$ \\
\hline & \multicolumn{9}{|c|}{ Same for all models } & \multicolumn{3}{|c|}{$\begin{array}{c}\text { MEVP-DS } \\
\text { S-CLAY1 } \\
\text { MCC }\end{array}$} & \multicolumn{4}{|c|}{ Same for all models } & $\begin{array}{l}\text { MEVP-DS } \\
\text { S-CLAY1 }\end{array}$ \\
\hline CAD2251 & Otaniemi & 16 & 20 & 1.0 & 0.67 & 0.3 & 2.46 & 0.001 & 1 & 0.42 & 20 & 0.50 & 0 & 0 & 0 & 1.2 & 0.73 \\
\hline CAD2261 & Otaniemi & 16 & 20 & 1.0 & 0.67 & 0.3 & 2.46 & 0.001 & 1 & 0.42 & 20 & 0.50 & 0 & 0 & 0 & 1.2 & 0.73 \\
\hline CAD2277 & Otaniemi & 16 & 18 & 1.0 & 0.67 & 0.3 & 2.86 & 0.001 & 1 & 0.42 & 20 & 0.50 & 0 & 0 & 0 & 1.2 & 0.73 \\
\hline CAD3524 & Otaniemi & 16 & 18 & 1.0 & 0.67 & 0.3 & 2.86 & 0.001 & 1 & 0.42 & 20 & 0.50 & 0 & 0 & 0 & 1.3 & 0.70 \\
\hline CAE2544 & Otaniemi & 16 & 22 & 1.0 & 0.67 & 0.3 & 3.79 & 0.001 & 1 & 0.30 & 20 & 0.67 & 0 & 0 & 0 & 1.3 & 0.70 \\
\hline CAE3215 & Murro & 16 & 35.5 & 1.0 & 0.67 & 0.2 & 1.99 & 0.001 & 1 & 0.46 & 20 & 0.67 & 0 & 0 & 0 & 1.6 & 0.65 \\
\hline CAE3820 & Otaniemi & 16 & 26 & 1.0 & 0.67 & 0.2 & 2.26 & 0.001 & 1 & 0.42 & 20 & 0.67 & 0 & 0 & 0 & 1.3 & 0.70 \\
\hline
\end{tabular}

Table 3.6 Compressibility parameters for MEVP-DS

\begin{tabular}{ccccc}
\hline Layer & $\boldsymbol{\lambda}_{\mathbf{0}}$ & $\boldsymbol{\lambda}$ & $\boldsymbol{\kappa}$ & $\boldsymbol{\zeta}$ \\
\hline & - & - & - & MEVP-DS \\
\hline MEVP-DS & S-CLAY1 and MCC & & 8 \\
CAD2261 & 0.27 & 0.44 & 0.04 & 10 \\
CAD2277 & 0.40 & 0.44 & 0.04 & 4 \\
CAD3524 & 0.38 & 0.44 & 0.04 & 6 \\
CAE2544 & 0.43 & 0.44 & 0.04 & 7 \\
CAE3215 & 0.28 & 0.44 & 0.04 & 4 \\
CAE3820 & 0.20 & 0.21 & 0.03 & 5 \\
\hline
\end{tabular}




\section{Determination of fundamental soil parameters}

Since the tests to be presented later were previously modeled using S-CLAY1 and MCC in the literatures, most of the model parameters can be conveniently taken except $t_{0}$. As creep is not considered in the simulation, $t_{0}$ has been assigned as 1 day. Soil structure is eliminated in this study too, hence, $\chi_{0}, \xi$ and $\xi_{d}$ are assigned zero.

\section{Determination of $\lambda_{0}, \varsigma$, and $M_{e} / M_{c}$}

MEVP-DS model employs the same $\lambda_{0}$ as MCC, which has to be defined from isotropic consolidation tests. However, IAC tests apply an anisotropic stress condition. To tackle this issue, an empirical approach can be used to estimate the $\lambda_{0}$ for each test. Recall that in Chapter 2 , the correlation has been arrived to relate $\lambda_{0}^{*}$ to $\eta_{H}$ :

$$
\lambda_{H}^{*}=e^{1.21 \frac{\eta_{H}}{M}} \lambda_{0}^{*}
$$

$\lambda_{0}$ can be thus obtained by $\lambda_{0}=\lambda_{0}^{*}\left(1+e_{0}\right)$. The input values for $\lambda_{0}$ are summarized in Table

Table 3.6. $\varsigma$ values that yield satisfactory fittings of $\varepsilon_{v} \sim \ln p^{\prime}$ are used for presented tests. In addition, for modeling extension tests, the $M_{e} / M_{c}$ ratio is estimated using

$$
\frac{M_{e}}{M_{c}}=\frac{3-\sin \varphi_{c}}{3+\sin \varphi_{c}}
$$

where $\sin \varphi_{c}$ is the critical stage frictional angle defined as

$$
\sin \varphi_{c}=\frac{3 M_{c}}{6+M_{c}}
$$

\subsubsection{SIMULATION OF IAC TESTS ON FINNISH CLAYS}

Figure 3.5 presents the simulation of CAD2251 using the newly proposed MEVP-DS model. The model prediction is compared with S-CLAY1 and MCC predictions. Since the yield surface anisotropy is considered by MEVP-DS and S-CLAY1, both models appear to accurately capture the yield stress when the soil is reloaded at the second stage, see Figure 3.5 (b). On the contrary, MCC is shown to overestimate the yield stress. 
Figure 3.5 (b) also shows MEVP-DS's capability of successfully predicting $\varepsilon_{v}$. It is demonstrated that MEVP-DS is able to reproduce soil compressibility corresponding to different stress ratios by considering deviatoric softening. In contrast, S-CLAY1 and MCC predict the same soil compressibility of two loading stages.

In addition, MEVP-DS also provides a better prediction of $\varepsilon_{d}$ as shown in Figure 3.5 (c), followed by S-CLAY1. The prediction with MCC is poor in both $\varepsilon_{v}$ and $\varepsilon_{d}$.
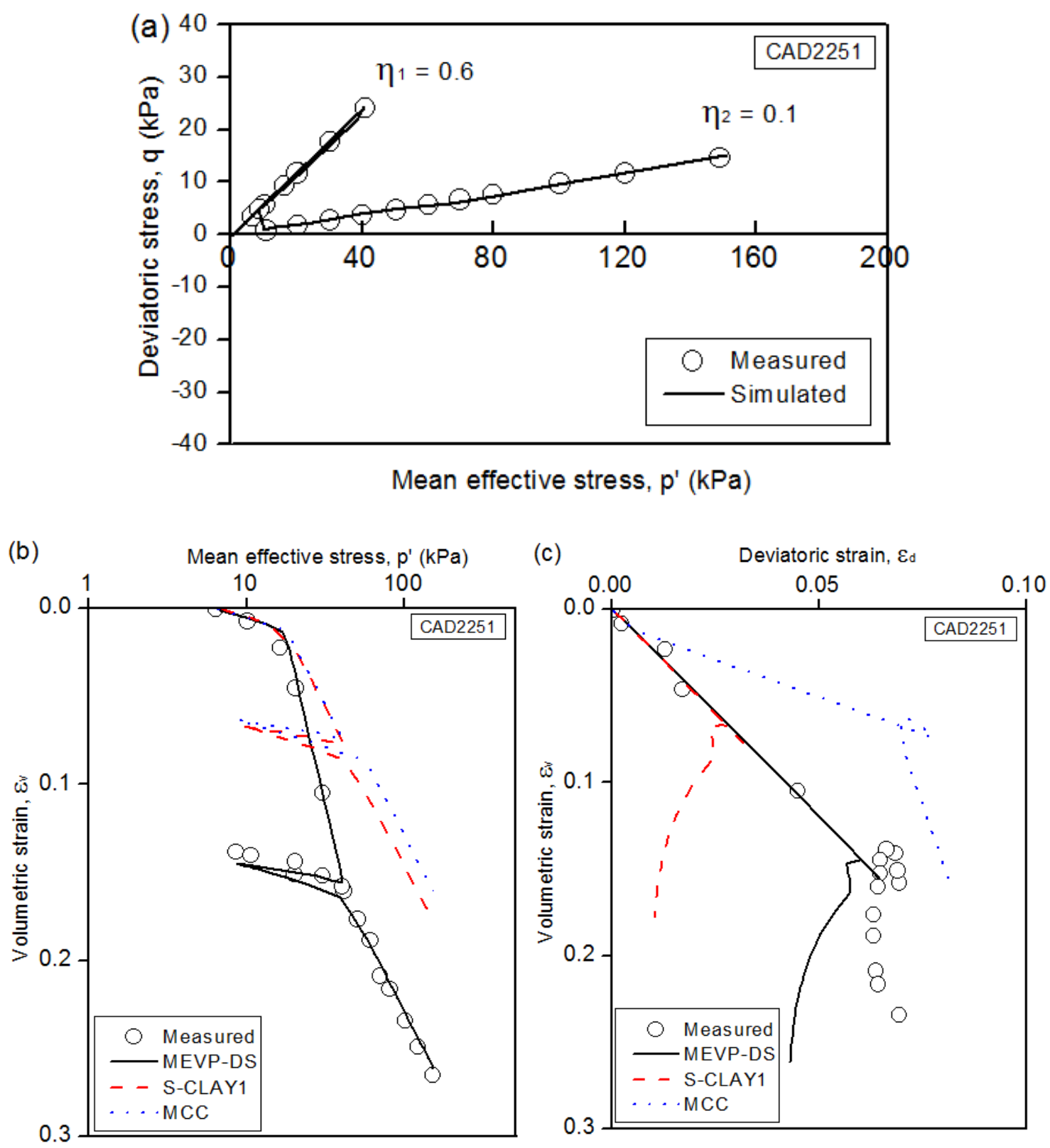

Figure 3.5 Model simulation of CAD2251 
Similar improvements by MEVP-DS can also be seen in Test CAD2277, shown in Figure 3.6 (b). MEVP-DS predicts the $\varepsilon_{v}$ and $\varepsilon_{d}$ much better than S-CLAY1 and MCC. MCC's prediction of $\varepsilon_{d}$ is rather deviant from the measurements. S-CLAY1's prediction of $\varepsilon_{d}$ is more reasonable than MCC, albeit some underestimation exists.
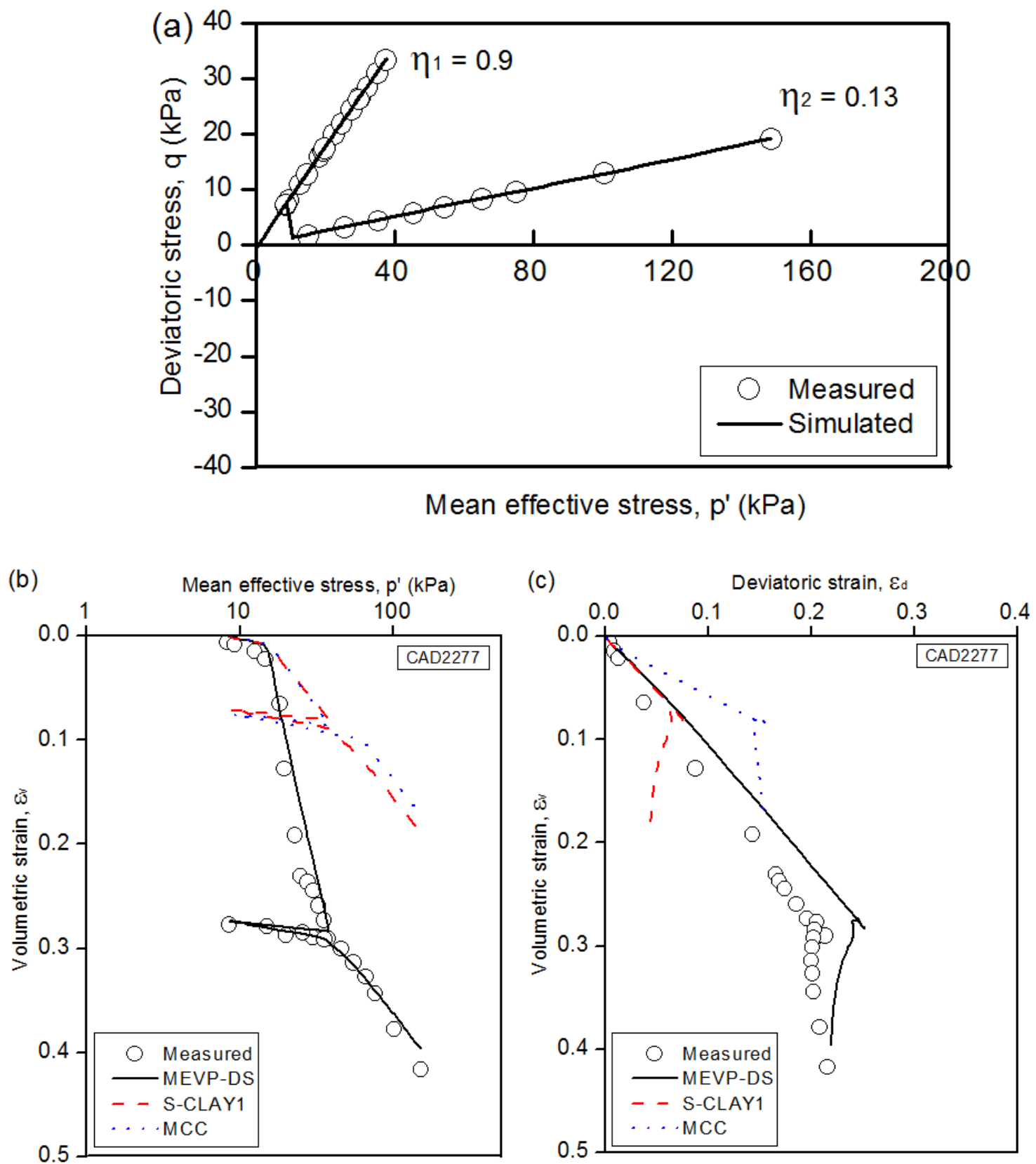

Figure 3.6 Model simulation of CAD2277 
For the extension test, CAE2544 (Figure 3.7), $\varepsilon_{v}$ has been inevitably under-predicted by SCLAY1 and MCC, whereas MEVP-DS well predicts $\varepsilon_{v}$ for both loading stages. In addition, MEVP-DS and S-CLAY1 well capture the yield stress when the sample is reloaded at $\eta_{2}=-0.59$. In contrast, MCC tends to over-predict the value, see Figure 3.7 (b).
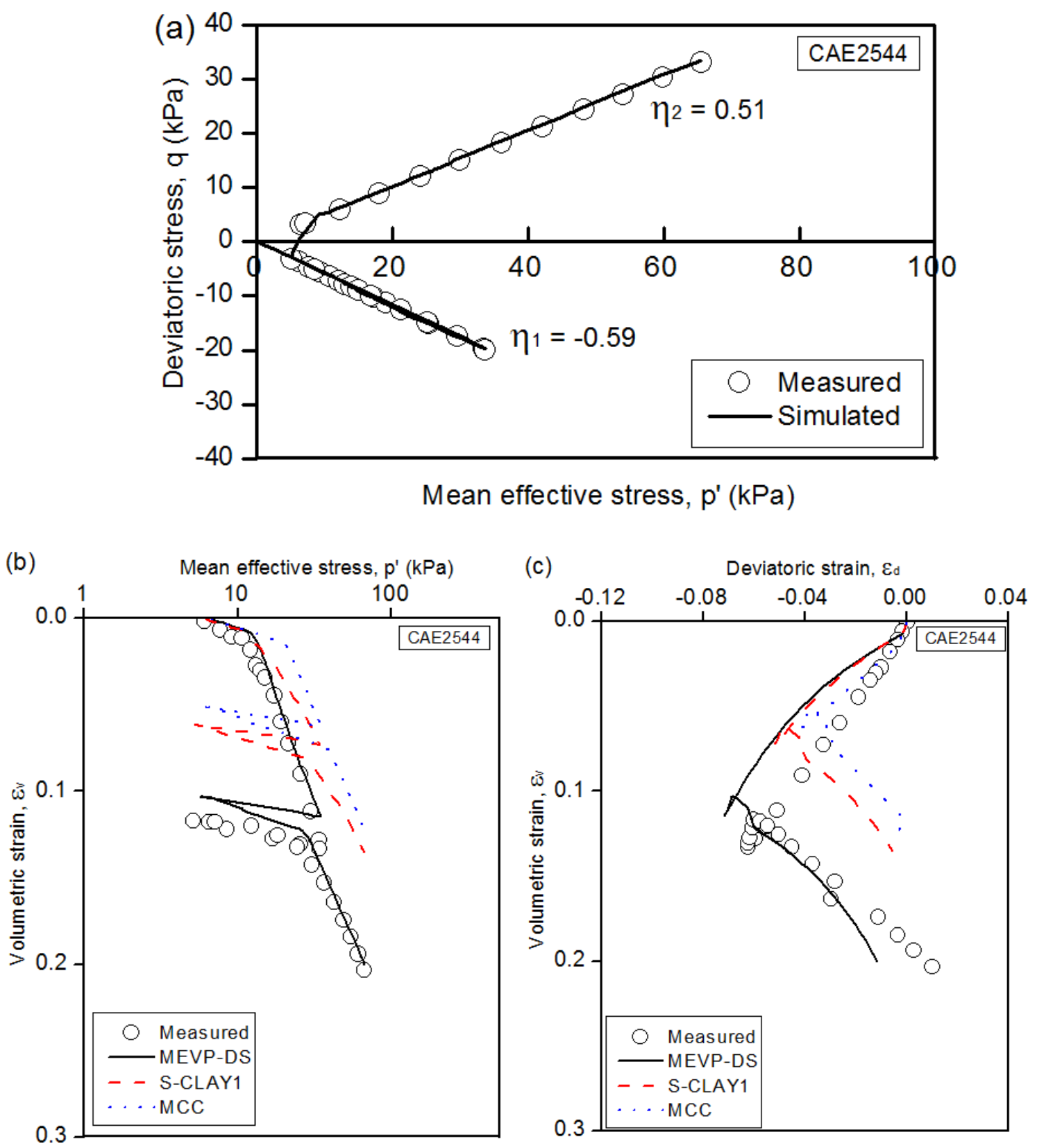

Figure 3.7 Model simulation of CAE2544 
CAE3215 involves some tremendous stress alterations from compression to extension, as shown in Figure 3.8 (a). All three models manifest various extent of discrepancy in predicting the yield stress when the soil sample is reloaded at $\eta_{2}=-0.41$. This is due to the difficulty in capturing rapid yield surface rotations when high positive stress ratios are reversed to negative (Wheeler et al. 2003). However, MEVP-DS still predicts the $\varepsilon_{\mathrm{v}}$ reasonably better than other two models. Figure 3.8 (c) and (d) present model simulations of $\varepsilon_{d} \sim \varepsilon_{v}$ and $\varepsilon_{d} \sim q$ relationship respectively. Similar observations can be seen for the simulation of CAE3820 in Figure 3.8.

(a)

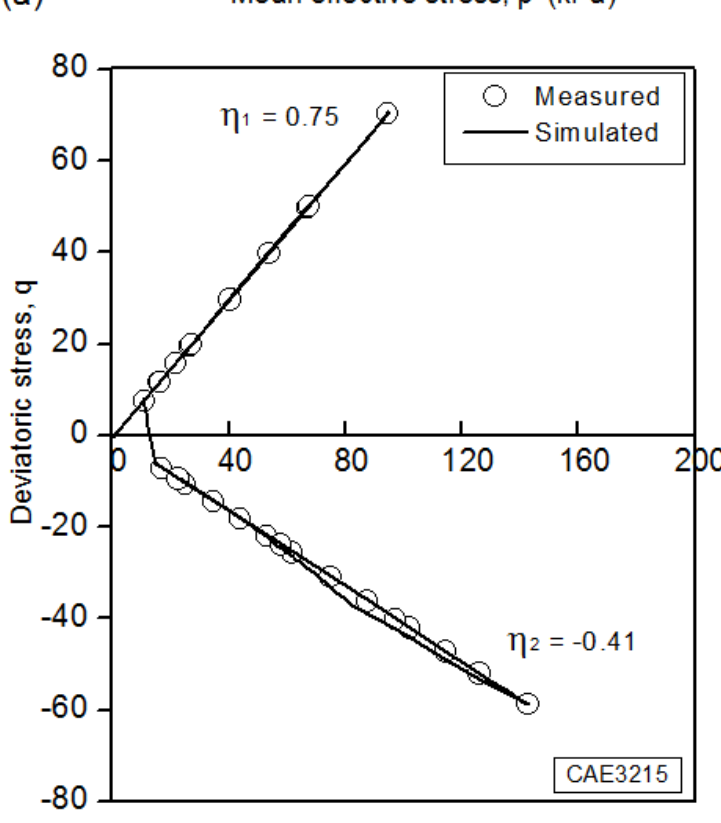

(b)

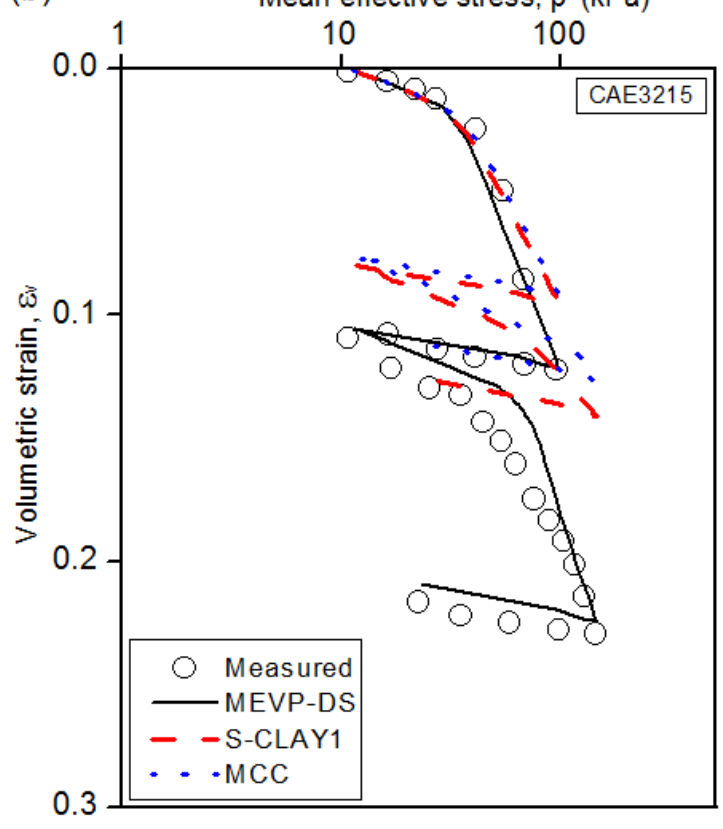



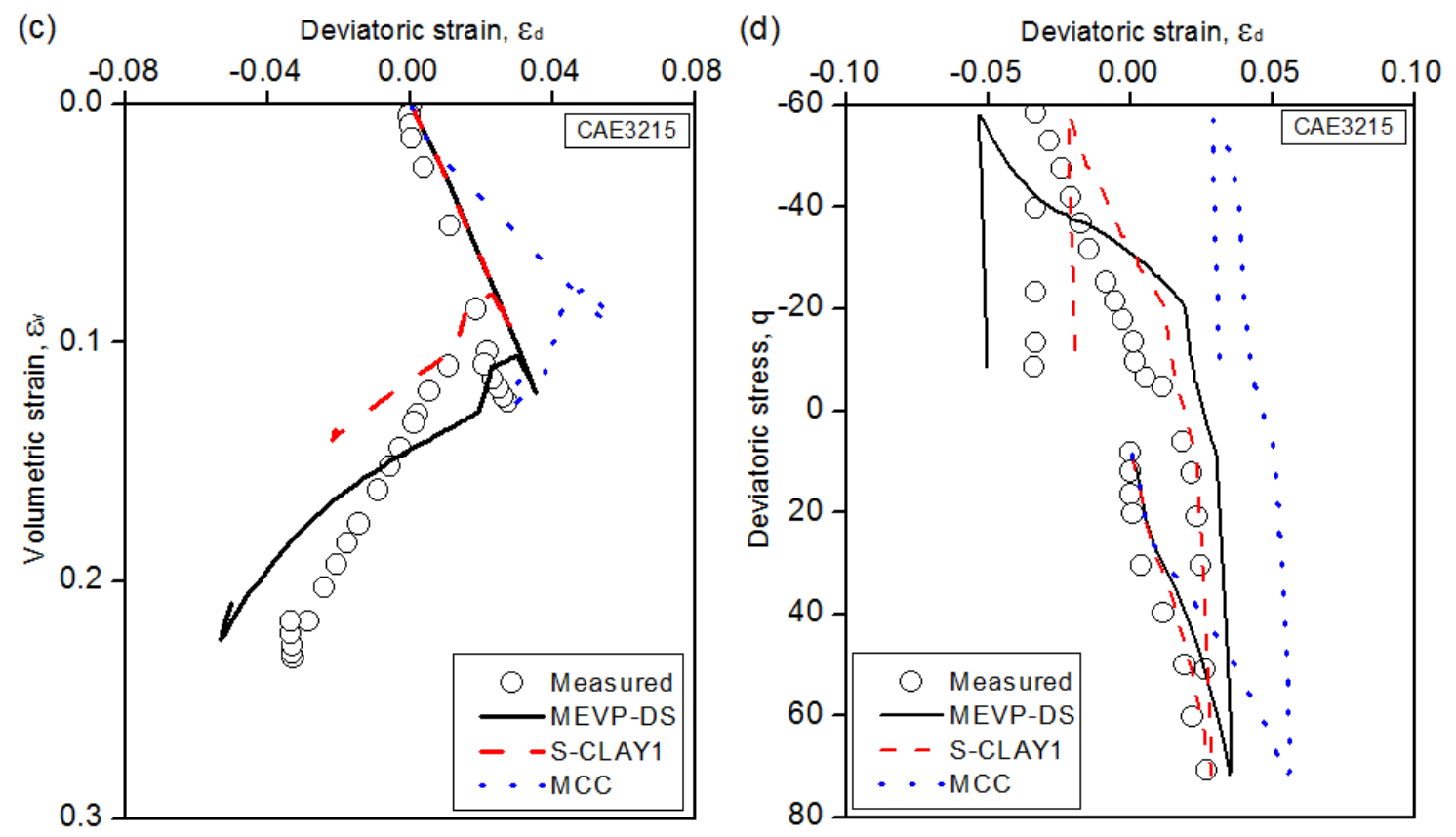

Figure 3.8 Model simulation of CAE3215 
(a)

Mean effective stress, $\mathrm{p}^{\prime}(\mathrm{kPa})$

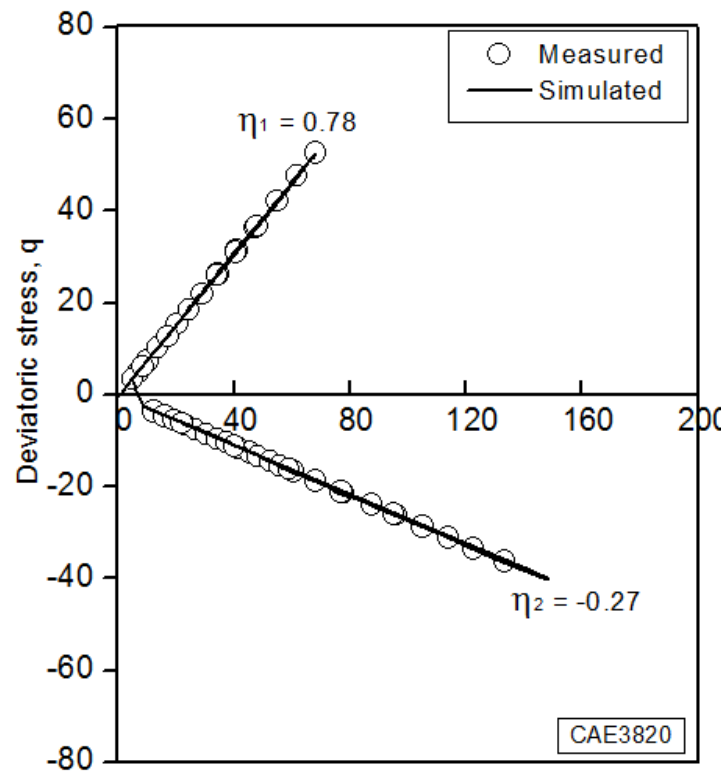

(c)

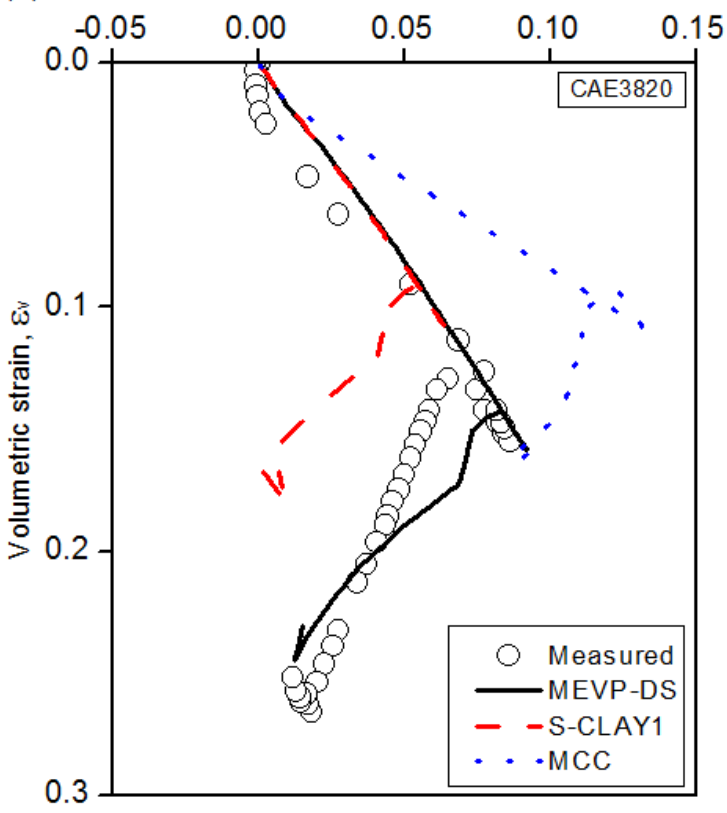

(b) Mean effective stress, $\mathrm{p}^{\prime}(\mathrm{kPa})$

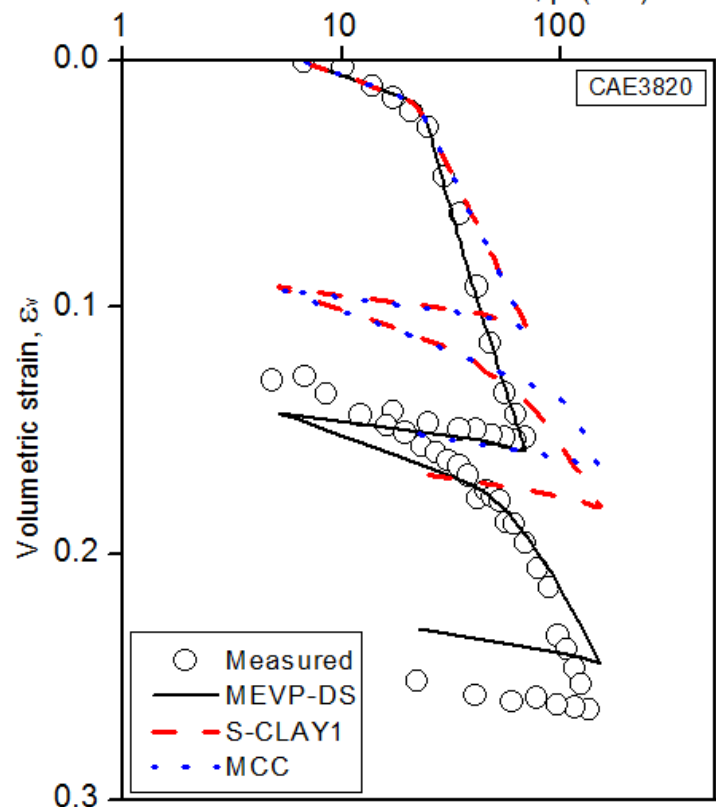

(d)

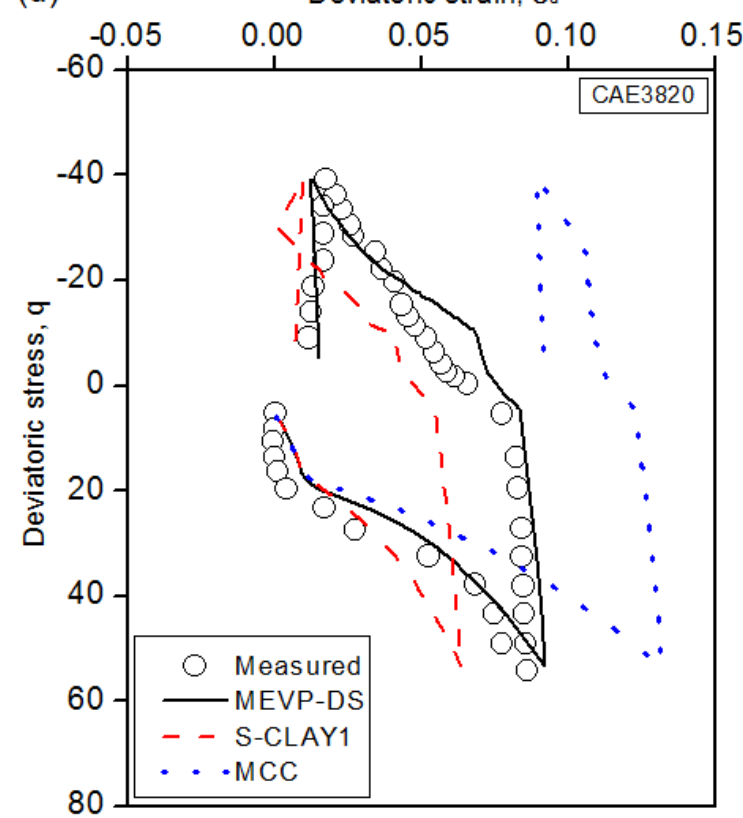

Figure 3.9 Model simulation of CAE3820 


\subsubsection{INFLUENCE OF SOFTENING FACTOR S}

This section aims to demonstrate how the values of $\varsigma$ for the simulation of IAC tests on Finnish clays are selected from trial modeling. By assigning various values of $\varsigma$ in trial models, the one that results in the overall best fitting of $\varepsilon_{v} \sim p^{\prime}, \varepsilon_{d} \sim \varepsilon_{v}$, and $\varepsilon_{d} \sim q$ prediction is considered as the selected value.

Equation [3.5] shows that the presence of the softening factor $\varsigma$ of MEVP-DS model will result in an increased $\varepsilon_{v}$. The sensitivity of $\varsigma$ is demonstrated by the simulation of Test CAD2261 (Figure 3.10) and CAD3524 (Figure 3.11). Two tests simulated by MEVP-DS indicate that $\varsigma$ needs to be calibrated by trial modeling to obtain the best fitting for each individual test. The prediction of both $\varepsilon_{v}$ and $\varepsilon_{d}$ grows with increasing values of $\varsigma$. The predicted $\varepsilon_{d} \sim \varepsilon_{v}$ relationships conclude that $\varsigma$ doesn't appear to affect the $d \varepsilon_{d} / d \varepsilon_{v}$ ratio, as represented by the parallel curves in Figure 3.10 (c) and Figure 3.11 (c), which should be mainly governed by $M$ and $\alpha$.

\subsection{CONCLUSIONS}

A modified deviatoric softening law is proposed assuming yield surface size softening is partially influenced by $d \varepsilon_{d}^{p}$. This softening law requires only one additional parameter, $\varsigma$, to govern the contribution of $d \varepsilon_{d}^{p}$ to yield surface softening. A new model, called MEVP-DS, has incorporated this softening law, along with yield surface anisotropy and destructuration of S-CLAY1S model, and Yin's EVP framework.

The verification of MEVP-DS is done through a series of simulations of IAC tests collected from literatures. The prediction of MEVP-DS model is compared with two simplified versions of MEVP-DS model equivalent to S-CLAY1 and MCC model.

The simulation results demonstrate that MEVP-DS provides an improved prediction of both $\varepsilon_{v}$ and $\varepsilon_{d}$ in contrast to S-CLAY1 and MCC. The parametric study shows that $\zeta$ influences both $\varepsilon_{v}$ and $\varepsilon_{d}$, but doesn't appear to influence $d \varepsilon_{d} / d \varepsilon_{v}$ ratio, which is governed by $M$ and $\alpha$. Some limitations of MEVP-DS model exist due to mathematical assumptions of the softening law: 
1. Model predictions of strains become sensitive to $\varsigma$ at high $\eta$ loading conditions. As the result, MEVP-DS may provide less accurate prediction when the soil sample is loaded at the $\eta$ close to $M$.

2. As seen in Chapter 2, the natural variation of investigated clays leads to the scattering correlation of $\frac{\lambda_{H}^{*}}{\lambda_{L}^{*}}$ and $\frac{\left|\eta_{H}\right|-\left|\eta_{L}\right|}{M}$ dependence. Consequently, it makes the accurate estimations of $\lambda_{0}$ difficult. The author suggests that some trial simulations be performed at first to calibrate $\lambda_{0}$ before simulating individual tests.

(a)

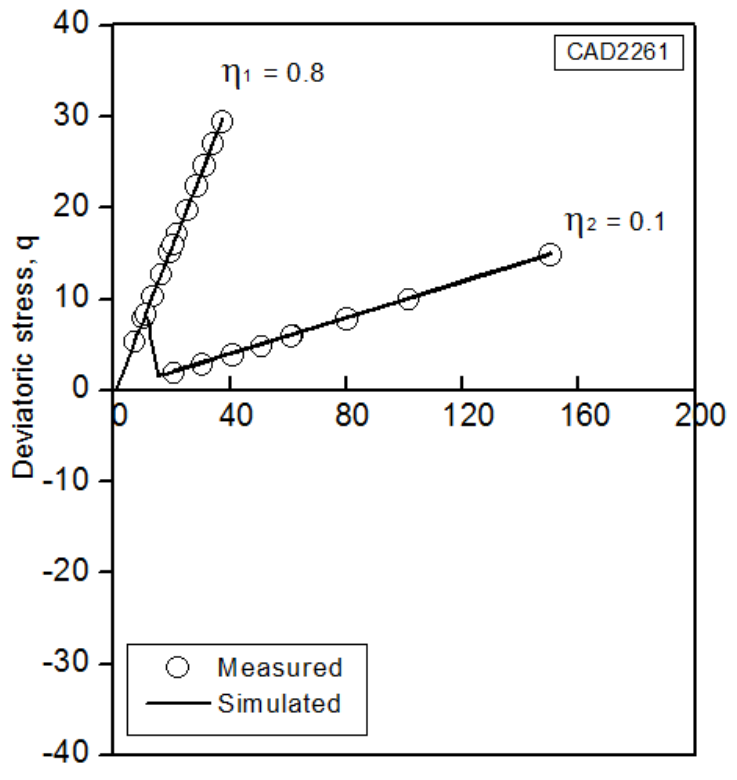

(b)

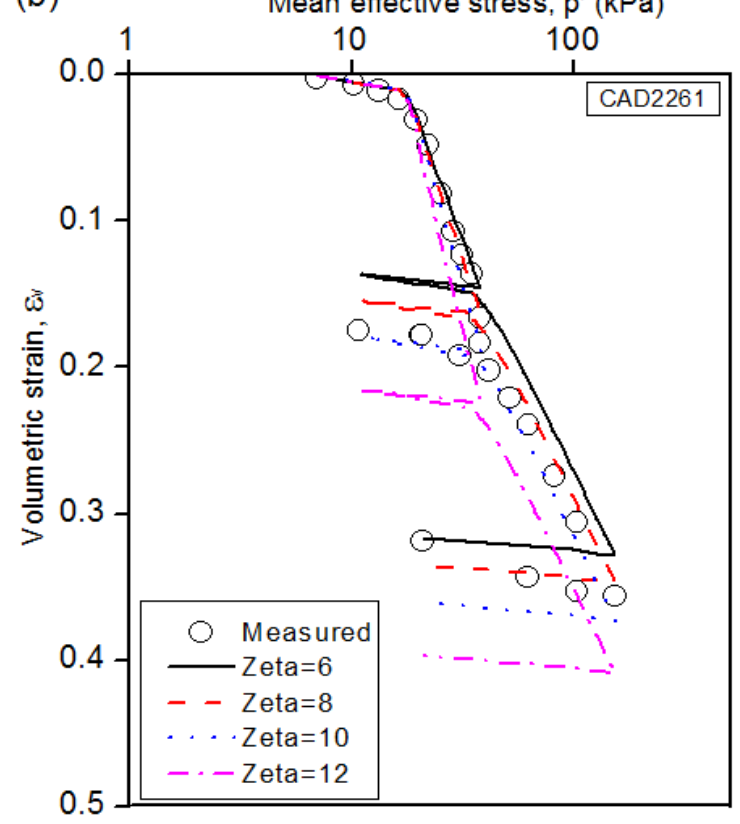



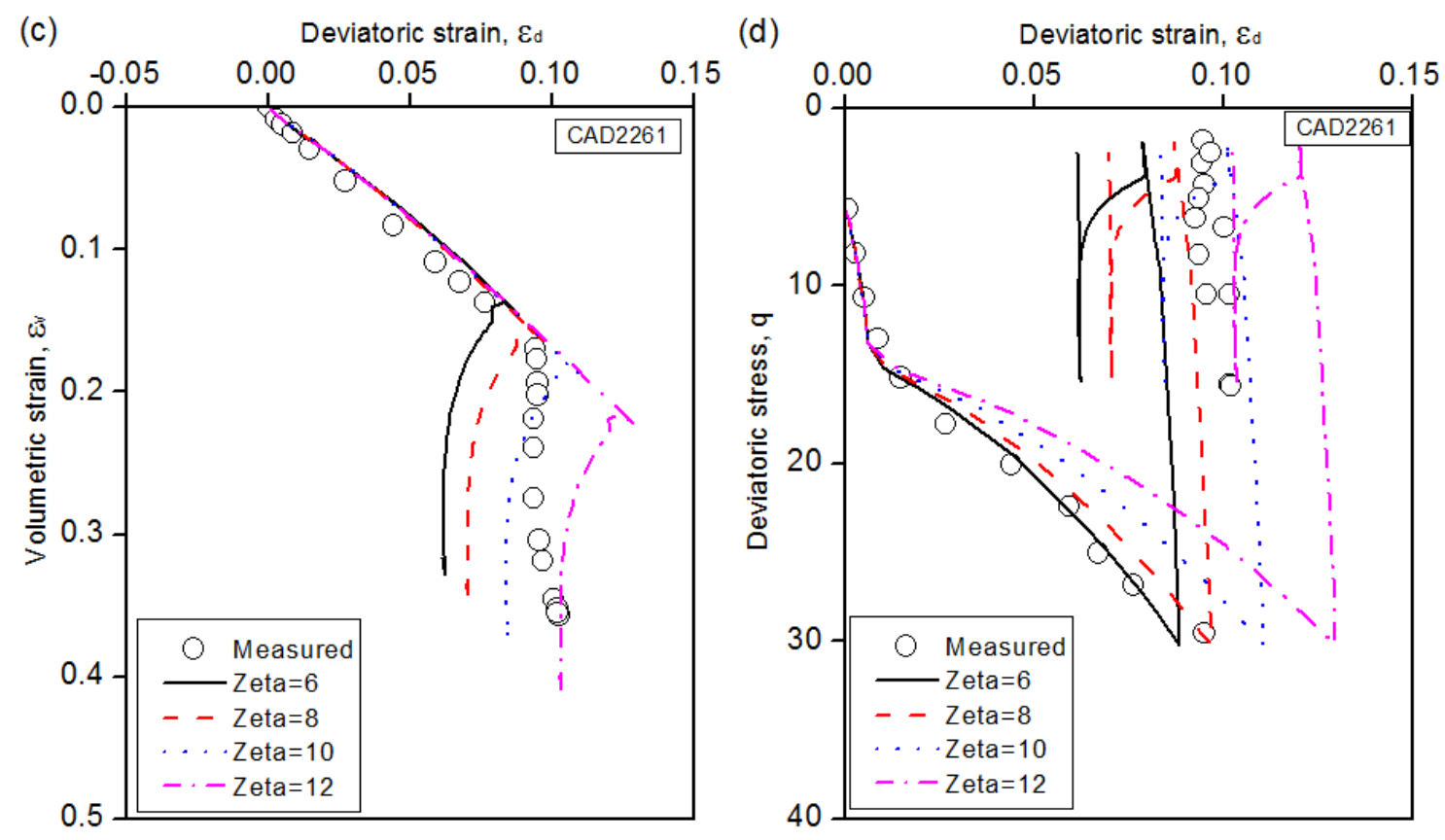

Figure 3.10 Sensitivity of $\varsigma$ to the simulation of CAD2261 
(a)

Mean effective stress, $\mathrm{p}^{\prime}(\mathrm{kPa})$

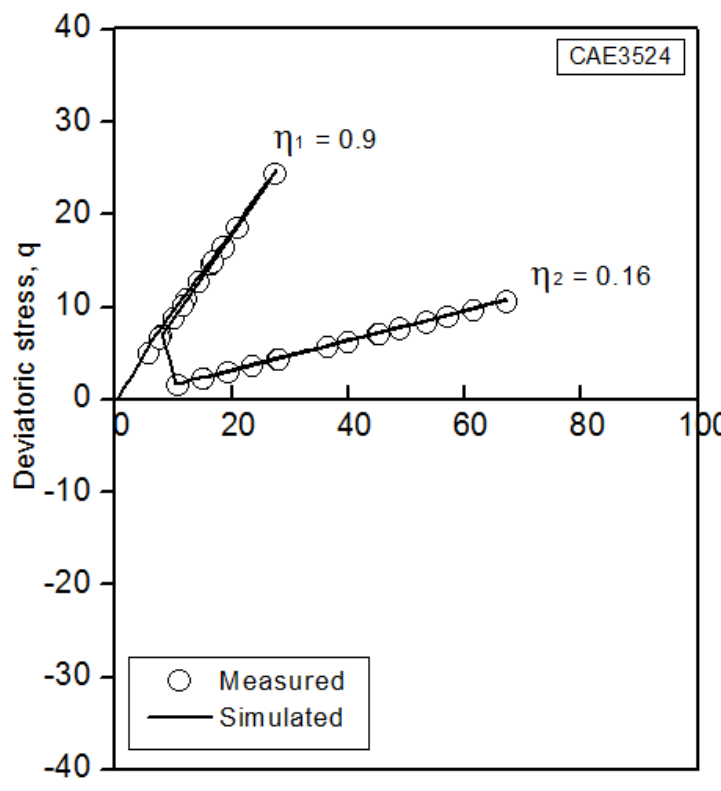

(c)

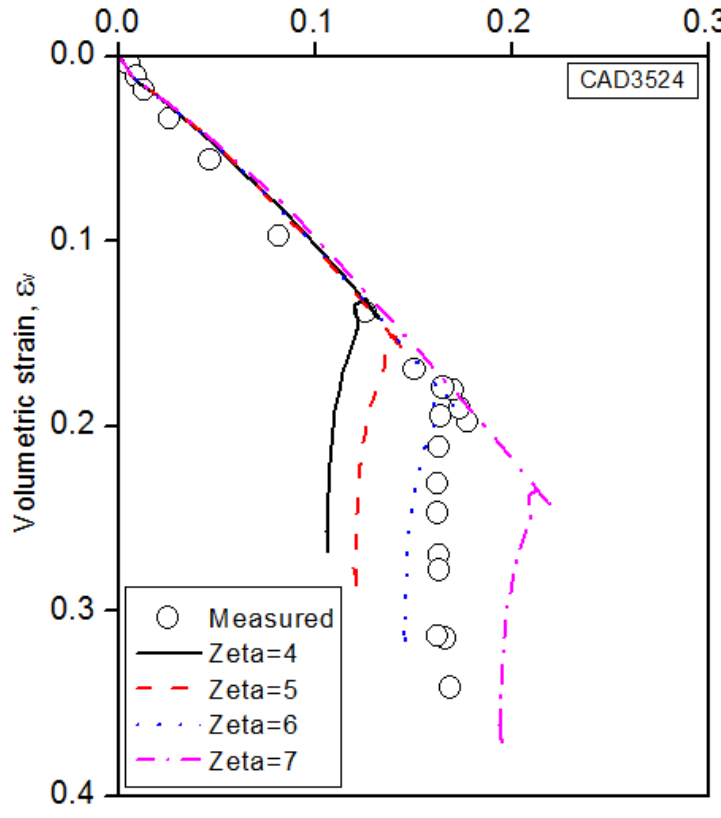

(b) Mean effective stress, $\mathrm{p}^{\prime}(\mathrm{kPa})$

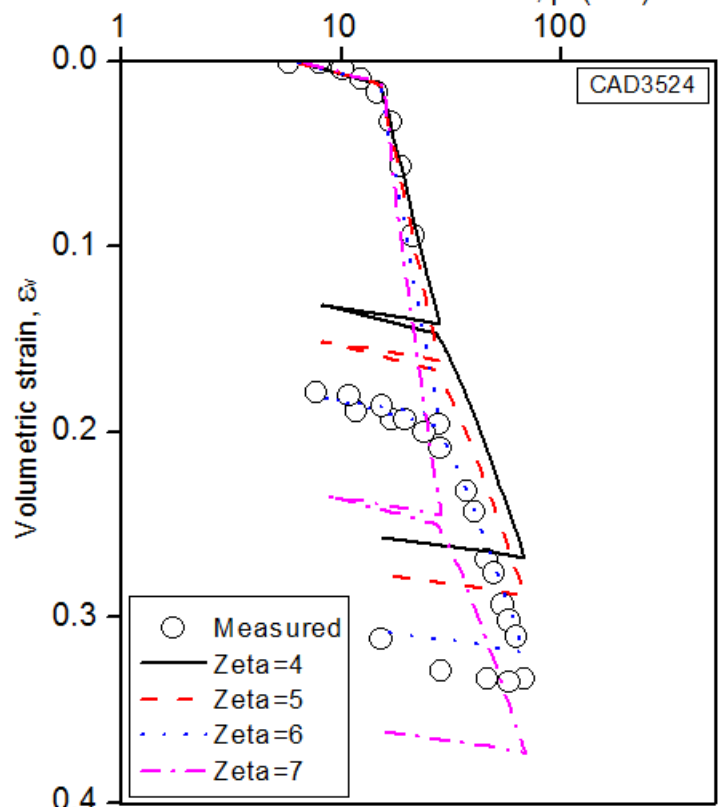

(d)

Deviatoric strain, $\varepsilon_{\mathrm{d}}$

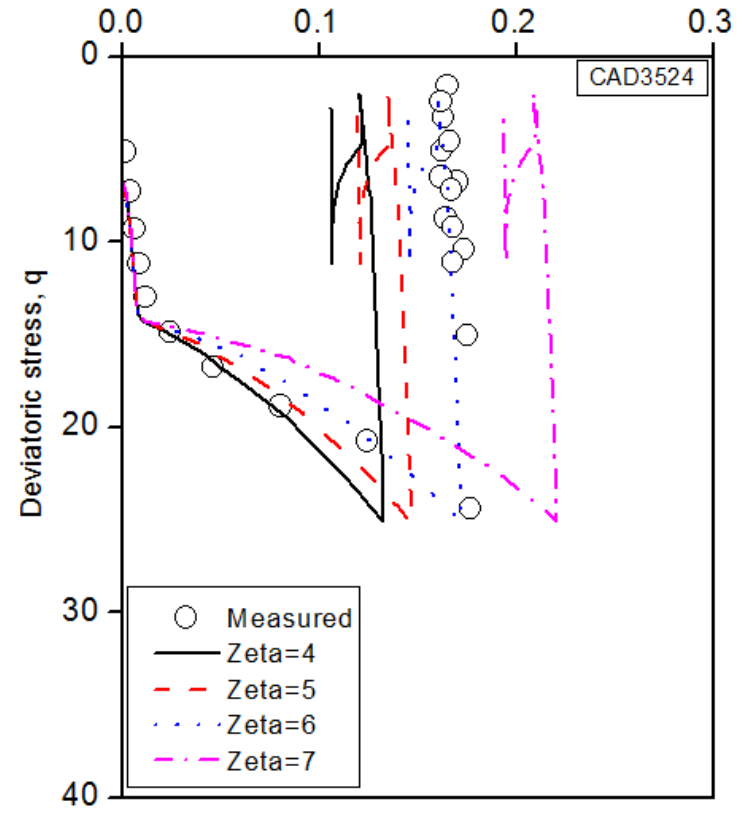

Figure 3.11 Sensitivity of $\varsigma$ to the simulation of CAD3524 


\section{MODEL VERIFICATION: MODELING 1-D COMPRESSION OF CHAMPLAIN SEA CLAY}

\subsection{INTRODUCTION}

In this chapter, the capacity of MEVP-DS model is validated through simulating a number of 1D consolidation tests conducted on Champlain Sea clay. Champlain Sea clay, which exhibits strong destructuration behavior, is a typical sensitive marine clay found in eastern Canada.

This chapter focuses on the modeling of constant rate of strain (CRS) tests. Both effectives stress and excess pore pressure response of Champlain Sea clay during CRS tests will be investigated. The capacity of MEVP-DS will be demonstrated by comparing its predictions with other models, including MEVP, S-CLAY1, S-CLAY1S, and SSC (Soft Soil Creep) model. Moreover, simulations of some conventional oedometer and 1-D creep tests will also be presented at the end of this chapter.

However, it is worth noted that the unloading response of Champlain Sea clay is not considered in CRS modeling. The correct simulation of the excess pore pressure response during unloadingreloading cycles still needs further study.

\subsection{THE FORMATION AND THE PROPERTIES OF CHAMPLAIN SEA CLAY}

\subsubsection{GEOLOGICAL HISTORY OF THE CHAMPLAIN SEA}

The Champlain Sea was a temporary inlet of the Atlantic Ocean and was at the maximum elevation over $200 \mathrm{~m}$ relative to the current sea level north of the St Lawrence and above $150 \mathrm{~m}$ above sea level south of the lowland (Quigley et al. 1983), see Figure 4.1 for its location.

The sea was formed due to the ablation during the closure of the last ice age around 8,000 to 10,000 years ago. At the time of the last ice age, a large area of eastern Canada, as well as part of New York, were depressed by glaciers. The area was also inundated by the sea water owing to worldwide rising sea level. 


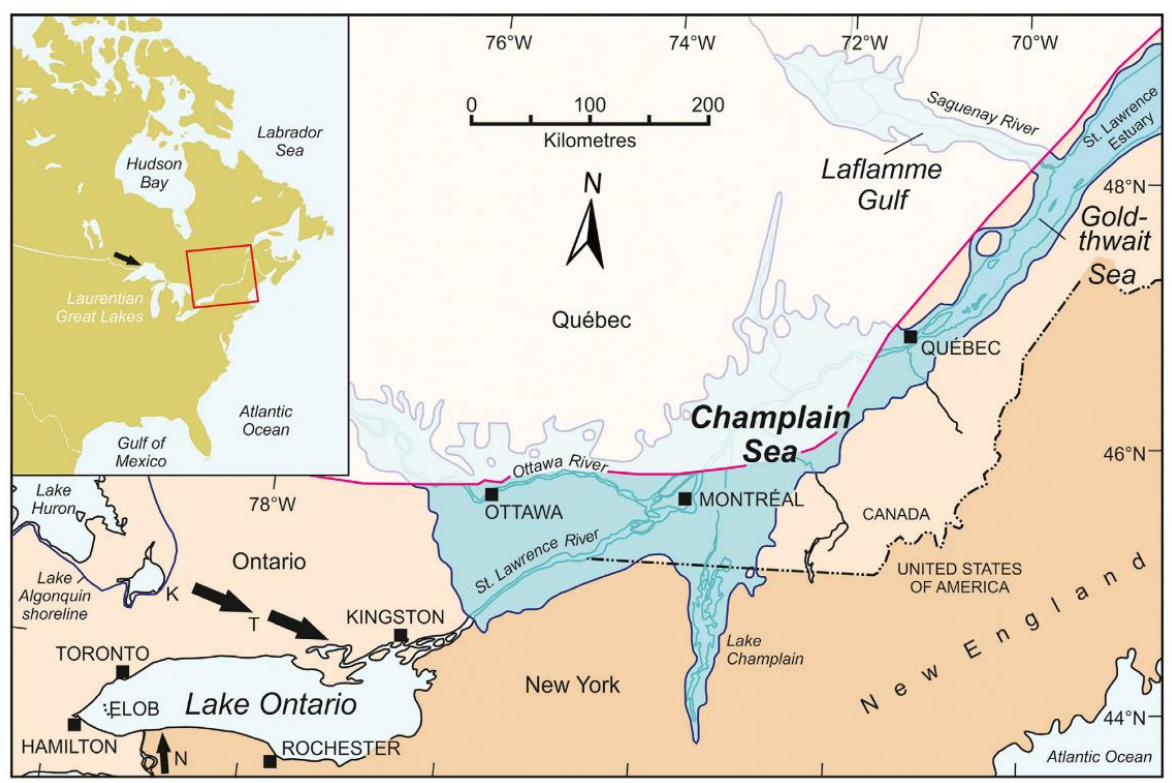

Figure 4.1 Regional map showing the extent of the Champlain Sea in the St. Lawrence River Valley, eastern North America. (Lewis and Todd 2019)

After glaciers retreated, the Champlain Sea clay started to form in a deep-water sedimentation process. Meanwhile, the land began to rise in the process of isostatic uplift, retreating the coast line to its current location. The Champlain Sea era was then terminated when the isostatic rebounding exceeded the eustatic rise in sea level.

The groundwater concentration analysis shows that the Champlain Sea water was a mixture of seawater $(33 \%)$ and fresh water (67\%) from precipitation and melting glaciers (Desaulniers and Cherry 1989, Cloutier et al. 2009). The marine nature of the Champlain Sea clay has been demonstrated by many species found, including but not limited to molluscs, barnacles, and several species of whales and seals.

The salinity level of the Champlain Sea clay is thought to vary from near-normal marine to relatively fresh. The pore water salinity determined in the Ottawa region is generally less than 2 $\mathrm{g} / \mathrm{L}$ (Gillott 1970), whereas a higher level of around $15 \mathrm{~g} / \mathrm{L}$ is noticed in Arnpior, Ontario (Liu et al. 2017). Brine samples retrieved in southern Ontario has a representative salinity of $11.3 \mathrm{~g} / \mathrm{L}$ (McNutt et al. 1987) 


\subsubsection{Formation OF CHAMPLAIN SEA CLAY}

The formation of marine clay usually involves a process called flocculation, where the sheet-like soil particles clump together to form a porous structure. Flocculation usually occurs in a brackish water condition, involving either edge to face or edge to edge flocculation, and is accompanied by cations swapping between soil particles. The highly porous structure of marine clay allows water to infiltrate, as seen in the scanning electron microscope (SEM) image in Figure 4.2.

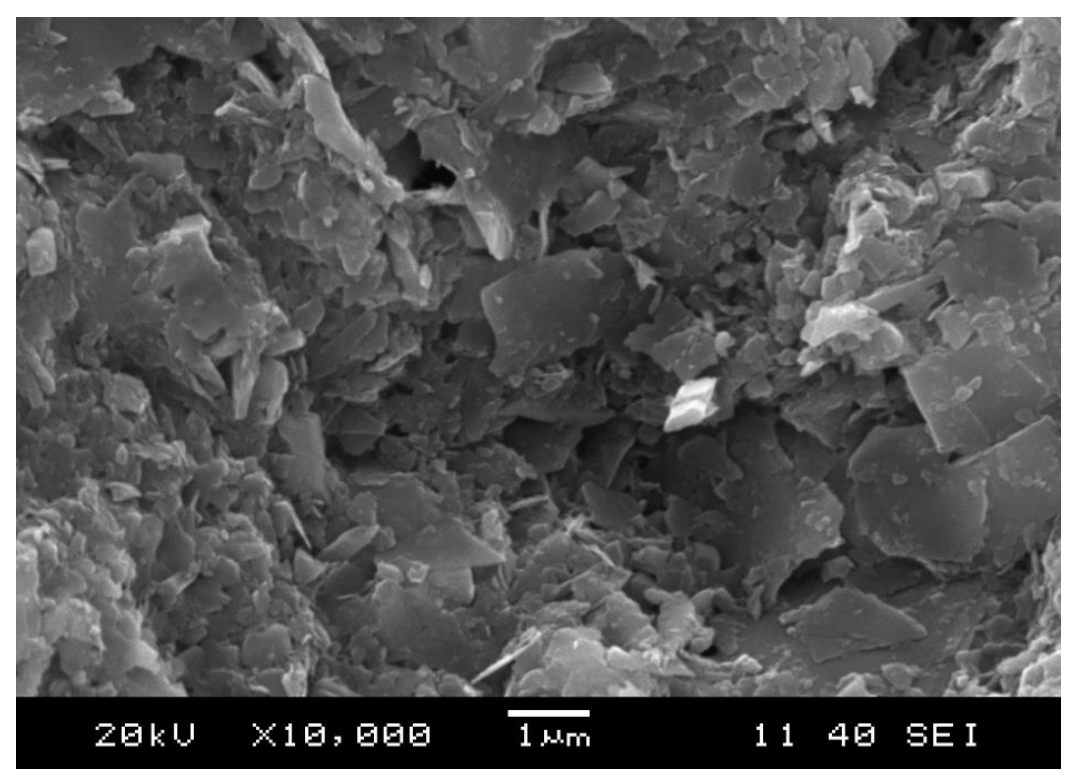

Figure 4.2 A SEM image of Champlain Sea clay (Monsif and Liu 2019)

One of the theories that helps explain the marine clay structure is the Double Layer (DL) theory (Figure 4.3). The theory assumes soil particles to be negatively charged, and surrounded by positive irons from disperse medium, such as water. This layer is called the stern layer, which is screened by the second positively charged iron layer called the diffuse layer. Irons that compose the diffuse layer are from free irons floating in the water. They become attached to the stern layer as the result of electric attraction and have much less bonding compared to the stern layer to particles. Outside the diffuse layer there is a slipping plane relative to a point in the bulk fluid away from the interface.

The electronic potential at the slipping plane, known as electrokinetic potential or Zeta potential, is the key indicator of the stability of marine clay particles in the water. The electrokinetic potential decreases exponentially with the distance moving away from a particle. Marine clays are 
composed of mainly fine particles in a micrometer or nanometer level so that the electrokinetic potential is usually strong to hold the soil structure stable. Study (Penner 1965) has shown marine clay sensitivity increases with electrokinetic potential.

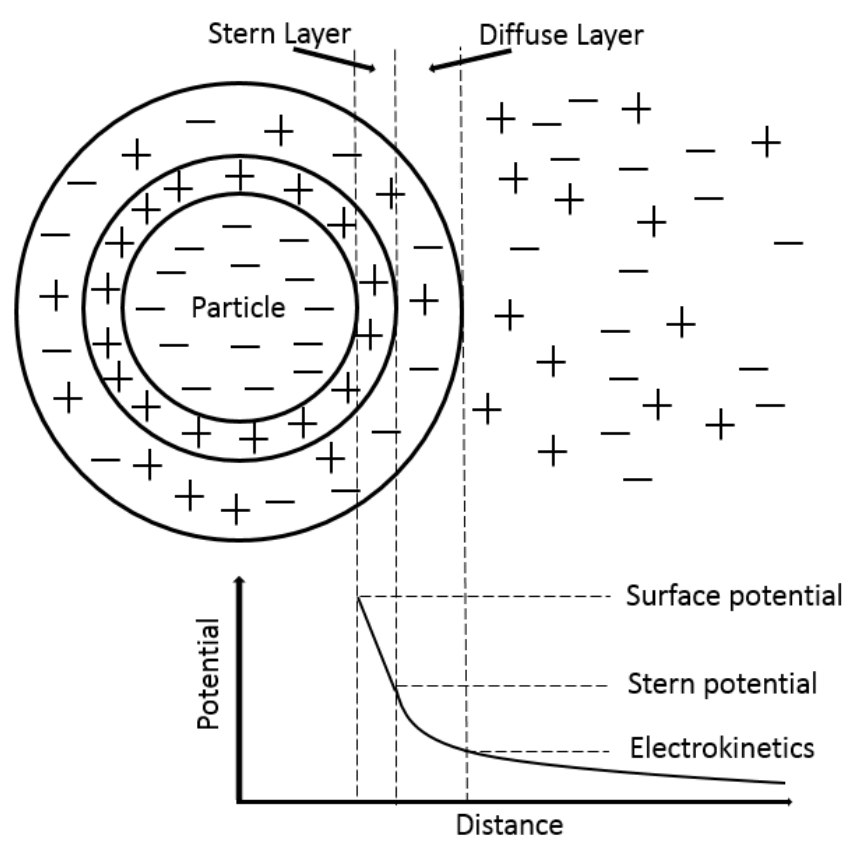

Figure 4.3 Electrical double layer theory and electrokinetics (Penner 1965)

The mineral content of Champlain Sea clay differs from regions, but quartz, feldspar, and plagioclase are present in a dominant proportion. Their mineral composition reflects the Precambrian rocks origin. The clay minerals are mainly illite and chlorite rather than expansive minerals such as montmorillonite. However, expansive clay minerals are found to dominate the surface layers in some Champlain Sea regions, which demonstrates its weathering and leaching nature (Nader 2014). As shown in Figure 4.4, the four main chemicals of Champlain Sea clay retrieved from Arnpiror, Ontario, are O (most >50\%), Si (> 20\%), Ai (10\%), and Fe (6\%) (Liu et al. 2017b). 


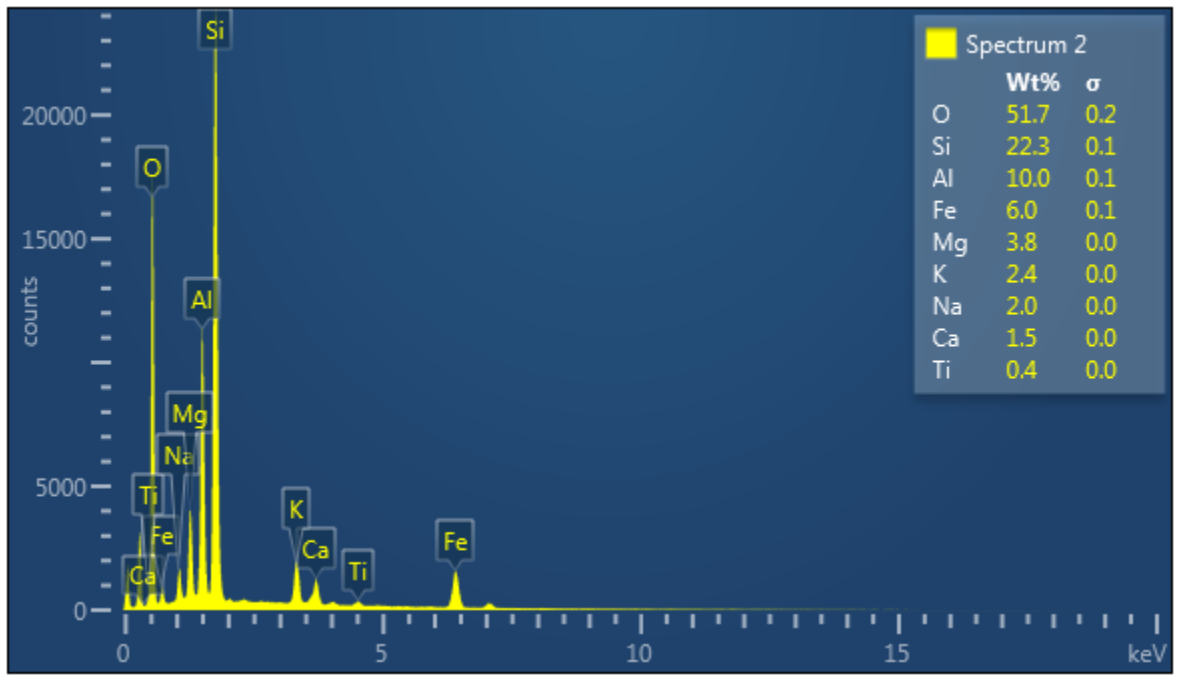

Figure 4.4 Chemical composition of Champlain Sea clay (Liu et al. 2017b)

\subsubsection{ENGINEERING PROPERTIES OF CHAMPLAIN SEA CLAY}

\section{Sensitivity}

Strong cementation bonding can grant undisturbed sensitive clay with a relatively strong shear strength. However, this fabric is susceptible to structure breakdown when high confining stress or shearing stress is applied, the process known as destructuration. Disturbing sensitive clays can also cause dramatic reduction in shear strength. Figure 4.5 presents a Champlain Sea clay sample turns into liquid consistency as the result of mix remolding for 3 minutes. This result has been observed at Ryerson geotechnical laboratory. Sensitivity, $S_{t}$, can be thus determined by dividing the undisturbed undrained shear strength by the remoulded undrained shear strength.

The typical sensitivity observed from the tested Champlain Sea clay at Ryerson University ranges between 7.2 and 14.9. A very high sensitivity is less reliable due to the difficulty in measuring the shear strength of very soft soil close to a liquid consistency. 


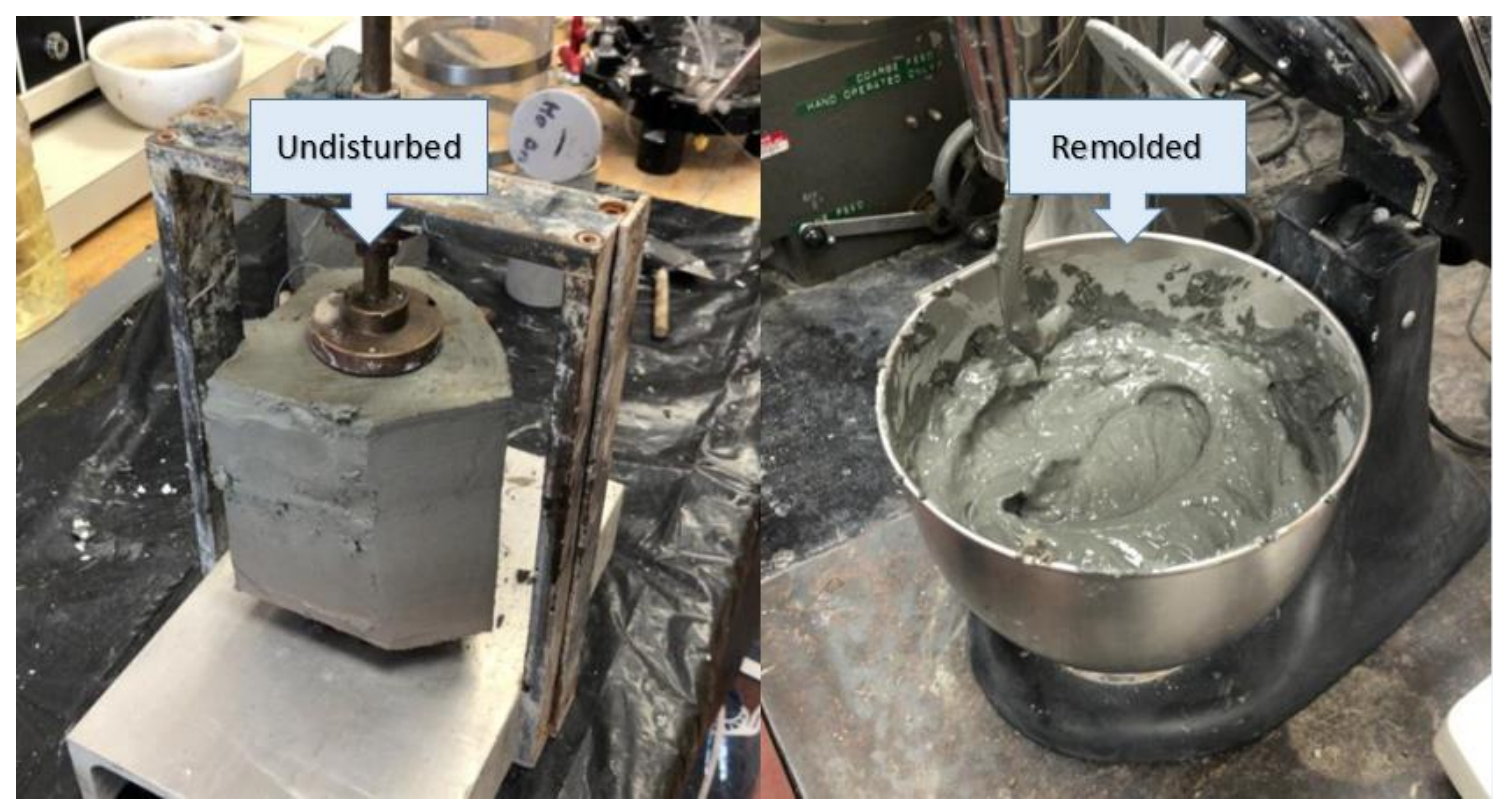

Figure 4.5 The loss of shear strength in Champlain Sea clay due to remolding

\section{Leaching}

The sensitivity of marine clay is associated with salinity of clay and the electrolyte concentration of pore water. Decreasing the salinity of marine clay can be achieved by leaching or diffusion. Leaching is often employed by generating a hydraulic gradient between two sides of a marine clay sample and flushing the sample with fresh water for a considerable amount of time. The leached Norwegian quick clays were reported to manifest dramatic increase in sensitivity from 8 to above 120 when salinity is reduced from $8 \mathrm{~g} / \mathrm{L}$ to less than $0.5 \mathrm{~g} / \mathrm{L}$ (Torrance 1974). Similarly, by diluting the Champlain Sea clay with gallons of fresh water, the recovered soil showed an increased sensitivity from 34 to 91 (Penner 1965).

However, increasing the sodium metaphosphate concentration of Champlain Sea clay was found to increase sensitivity dramatically. As reported in (Penner 1965), the sensitivity of a Champlain Sea clay from Ottawa has surged from 91 to 2150 when $1 \mathrm{~g}$ of sodium metaphosphate was added per $100 \mathrm{~g}$ of soil.

Leaching has also been found to influence the compressibility of Champlain Sea clay from Ryerson geotechnical laboratory. A higher compressibility was recorded from the remoulded 
leached sample. In addition, when leached clay is treated with cement, a lower compressibility has been obtained in cement treated samples at a lower salinity level (Ahmad 2018).

\section{Landslides}

The dramatic shear strength reduction of Champlain Sea clay when subject to disturbance has been proved to cause landslides. It has been associated with more than 250 reported landslides in eastern Canada. Examples include Saint-Maurice landslide in 1663, Saint-Alban in 1890 and Nicolet landslide in 1955. The Champlain Sea clay associated slope failures reported in Ottawa Area from 1960-1970 shared an inclination of $24^{\circ}$ to $35^{\circ}$, many of which were induced by precipitations in the combined form of melted snow, heavy rainfalls and toe erosion (Eden and Mitchell 1970).

\section{Settlement}

The open fabric structure of marine clay allows additional share of water to exist between soil particles. This state is considered as meta-stable in that it is distinct from the structure of the uncemented normally consolidated soil. When loaded with a stress higher than its previous overburden pressure, the soil fabric starts to degrade, featured by the stacking of the plateletshaped soil particles. Subsequently, substantial volumetric deformation will occur.

The $v \sim \log p^{\prime}$ curves of the Champlain Sea clay samples from oedometer tests often manifest an abrupt collapse in the vicinity of the pre-consolidation pressure. Structures built on Champlain Sea clay foundations are thus susceptible to settlement issues, such as the National Museum Building in Ottawa (Crawford 1953, Crawford and Bozozuk 1990), embankments of Ottawa Queensway (Burn and Hamiltion 1968, Burn 1969) and so forth.

Creep, referring to slow deformation of materials as a result of prolonged pressure and stress, accounts for a noticeable proportion of settlement when evaluating the long-term deformation of Champlain Sea clay. This type of volumetric compression prevails in the virgin compression range and is not associated with excess pore pressure dissipation as revealed in laboratory samples. Creep strains contain both volumetric and deviatoric strain components and can be often observed in undisturbed over-consolidated Champlain Sea clay samples (Burn 1969, Mitchell 1970).

\section{Over-consolidation and $K_{0}$}


Weathering, aging, leaching, and unloading in the geological history are thought to have resulted in the over-consolidation of surface layers in many areas in the Champlain Sea region (Quigley et al. 1983). For this reason, over-consolidation ratios (OCR) of these regions can reach as much as 5. OCRs are found to decrease with depths in Champlain Sea clay foundations. At some sites, OCRs have been observed to rise again when close to the deep area of deposits.

Retrieving a perfectly undisturbed Champlain Sea clay sample is nearly impossible as the clay is susceptible to disturbance. Hence, the conventional laboratory determination may underestimate the in-situ pre-consolidation (Burn and Hamiltion 1968). On the other hand, the mobilized preconsolidation pressure, as proposed by Leroueil et al. (1983a), can be determined by conventional oedometers on good quality samples taken with a $70 \mathrm{~mm}$ diameter piston sampler.

The $K_{0}$, the coefficient of earth pressure at rest, has been found in many Champlain Sea clay sites (Lefebvre et al. 1991, Hamouche et al. 1995). In the referred studies, the in-situ $K_{0}$ value were obtained by the means of Cambridge self-boring pressuremeter (Mark VIII model), the hydraulic fracturing, and the Marchetti's dilatometer.

An empirical relationship of $K_{0}=K_{0 n c} O C R^{0.95 \sim 0.98}$ have been agreed for Champlain Sea clay. This correlation gives a higher estimation of $K_{0}$ than the widely used correlation of $K_{0}=(1-$ $\left.\sin \varphi^{\prime}\right) O C R^{\sin \varphi^{\prime}}$ (Mayne and Kulhawy 1990). Moreover, 24h oedometer tests on Champlain Sea clay also demonstrate that there is no parallel between the horizontal and vertical yield stress ratio $\sigma_{h}^{\prime} / \sigma_{v}^{\prime}$ and $K_{0}$, as two values are found to be independent (Hamouche et al. 1995).

In addition, $\sigma_{h}^{\prime} / \sigma_{v}^{\prime}=0.73$ for Champlain Sea clay in Arnprior, Ontario, has been suggested by Ryerson geotechnical laboratory from a series of CRS tests on the undisturbed samples cut in both horizontal and vertical directions (Kirstein 2017).

\subsection{BACKGROUND OF CRS CONSOLIDATION TEST}

\subsubsection{INTRODUCTION TO CRS TESTS}

Understanding compressibility and consolidation properties is crucial to settlement predictions of structures built on soft clay foundations. These properties are often measured by conventional 
one-dimensional consolidation tests, or oedometer tests. Incremental loads are applied to soil samples on a 24 hour interval basis and thus $v \sim \log p^{\prime}$ can be obtained.

Nevertheless, the density of data points yielded by oedometer tests are dependent on the use of load increment ratio (LIR), which is often taken as one. This practice results in a large spacing between data points and may lead to a poor determination of coefficient of compression, preconsolidation pressure, and other consolidation parameters.

For sensitivity clay, oedometer tests have been shown to yield unsatisfactory results as the lack of resolution of data fails to fully capture the detail of structure collapse right after the preconsolidation pressure. To ensure the sufficient amount of data points, one would reduce LIR to 0.5 (Silvestri 1986). However, this will prolong the duration of tests dramatically, as a typical oedometer test of LIR $=0.5$ takes an average of two weeks to finish.

To improve the resolution of test data and meanwhile speed up tests, some alternative 1-D consolidation tests have thrived, such as controlled-gradient consolidation tests (Lowe et al. 1969), controlled-stress consolidation tests (Aboshi et al. 1970) and CRS tests (Smith and Wahls 1969).

CRS consolidation tests are widely used nowadays as an alternative of incremental loading (IL) consolidation tests. The test applies controlled strain rates to soil samples and measures stress responses. Excess pore pressure can be also measured during CRS tests. CRS tests are considered efficient in that (1), much higher density of data points available and faster test speed, (2) controlled strain rate at both loading and unloading stages, and (3) back pressure is designed for sample saturation.

Various consolidation theories for CRS have been developed for the calculation of permeability $(k)$ and coefficient of consolidation $\left(c_{v}\right)$. Most popular theories include the linear theoriy (Smith and Wahls 1969) and non-linear theory (Wissa 1971). The linear theory assumes a constant coefficient of volume compressibility $m_{v}$ defined as $\frac{d \varepsilon}{d \sigma^{\prime}}$. Wissa's non-linear theory assumes a constant compression index $\left(C_{c}\right)$ and provides a complete solution of the linear theory. The advantage of Wissa's theory is that it differentiates the transient condition from the steady condition. The trainsient condition is thought to emerge during early state of tests and the steady condition occurs afterwards.

The average effective stress defined in Wissa's non-linear theory is 


$$
\sigma_{v}^{\prime}=\sigma_{v}-\frac{2}{3} \Delta u_{b}
$$

The schematic drawing of the general-purpose consolidometer developed by MIT is shown in Figure 4.6

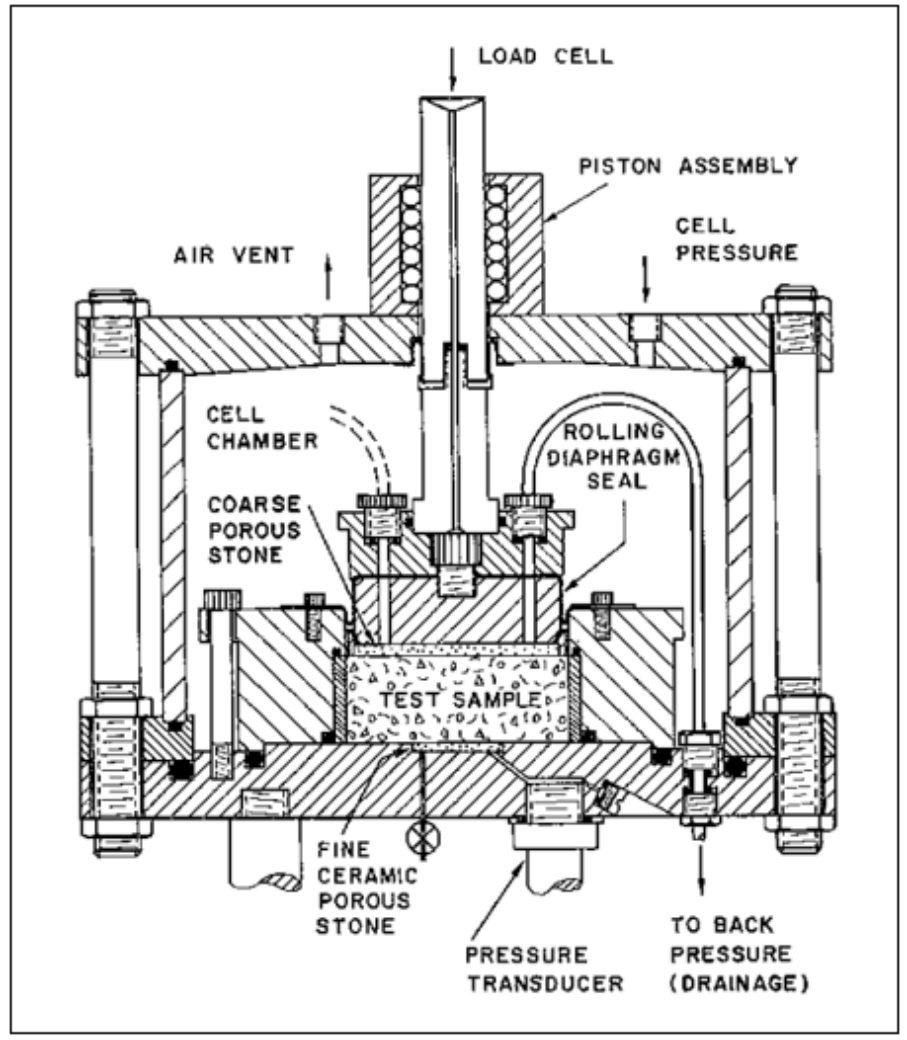

Figure 4.6 MIT general-purpose CRS apparatus (Wissa 1971)

The test sample is first cut into a lubed steel-walled retaining ring. The ring fits into a heavierwalled ring which provides additional support to prevent lateral deformation. The rings and samples are placed in the specimen chamber, hydraulically isolated from the cell chamber.

At the bottom of the sample ring places a fine ceramic porous stone to allow the drainage. Two drainage tubes are connected to the bottom and entrapped air can be flushed out by applying back pressure of 50 psi to 100 psi. Pore pressure is captured by the pressure transducer and volume change is monitored by an apparatus installed on the top of the sample ring. Vertical displacement is applied by the piston at a designed constant strain rate. 


\subsubsection{STRAIN RATE INFLUENCE ON CRS TEST RESULTS}

Strain rate can be a key factor influencing CRS test results. The reason is that rates used in CRS tests can be several magnitudes higher than conventional oedometer tests. As such, the preconsolidation pressure obtained from a typical $10^{-5} / \mathrm{s}$ of CRS tests, may be $30 \%$ to $50 \%$ higher than the one determined from oedometers, which have a much lower strain rate of $10^{-7} / \mathrm{s}$ (Leroueil et al. 1983b, Silvestri 1986).

During the loading stage of a CRS test, the top surface of a soil sample is opened for drainage. Howver, the bottom surface is kept undrained, and thus excess pore pressure is generated at the bottom. The amount of excess pore pressure should be controlled by allowable strain rates applied. The ratio of base excess pore pressure to total vertical stress $R=\mathrm{u}_{\mathrm{b}} / \sigma_{\mathrm{v}}$ has been used by many (Larsson and Sallfors 1985, Sheahan and Watters 1997, ASTM 2015) as a criterion to determine the allowable strain rate for tests. However, the suggested $R$ in different literatures fall into a wide range from 0.03 to 0.7 depending on various soil types.

Based on CRS test results, Ahmadi et al. (2014) reported that $\mathrm{u}_{\mathrm{b}}$ in non-sensitive clays is approximately linearly related to $\varepsilon_{v}$ regardless of the plasticity of clays. However, the experimental study on a sensitive clay in Eastern Canada, involving both CRS tests and constantgradient tests, demonstrated a non-linear relationship between $\mathrm{u}_{\mathrm{b}}$ and $\varepsilon_{v}$ (Silvestri 1986). It can be explained by a number of factors, including but not limited to strain rate, soil plasticity, organic content, soil compressibility, and permeability (Reddy et al. 2015). It's also been demonstrated that destructuration is the key factor of $\mathrm{u}_{\mathrm{b}}$ generation in Champlain Sea clay (Shi and Salloum 2018).

\subsubsection{CRS TESTING SYSTEM AT RYERSON UNIVERSITY AND SAMPLE PREPARATION}

A series of CRS tests were conducted on undisturbed clay samples according to ASTM standard D4186-06 (ASTM 2008). The test setup can be seen in Figure 4.7. The CRS machine used in the testing program was manufactured by Trautwein Soil Testing Equipment Company. Figure 4.8 shows the consolidation cell with all connections of sensors and tubes. 
To prepare soil sample for the test, the sample chunk was firstly trimmed carefully into a CRS ring with a diameter of $6.35 \mathrm{~cm}$ and a height of $2.54 \mathrm{~cm}$ (Figure 4.9). A porous stone was placed on the top to allow the consolidation drainage, while the bottom was kept undrained.

After the CRS ring was put into the cell, a back pressure of about $350 \mathrm{kPa}$ was applied for about 24 hours to saturate the sample. Then, an axial load was applied from the top allowing the sample to deform at the assigned constant strain rate. The force reaction and excess pore pressure response were captured by the load cell and the pore pressure sensor throughout the test. Strain rates of 0.5 to $1 \% / \mathrm{hr}$ were selected to ensure the ratio $R$ remains between $3 \%$ and $15 \%$ during CRS tests. More procedures and data processing details can be found in Kirstein (2017).

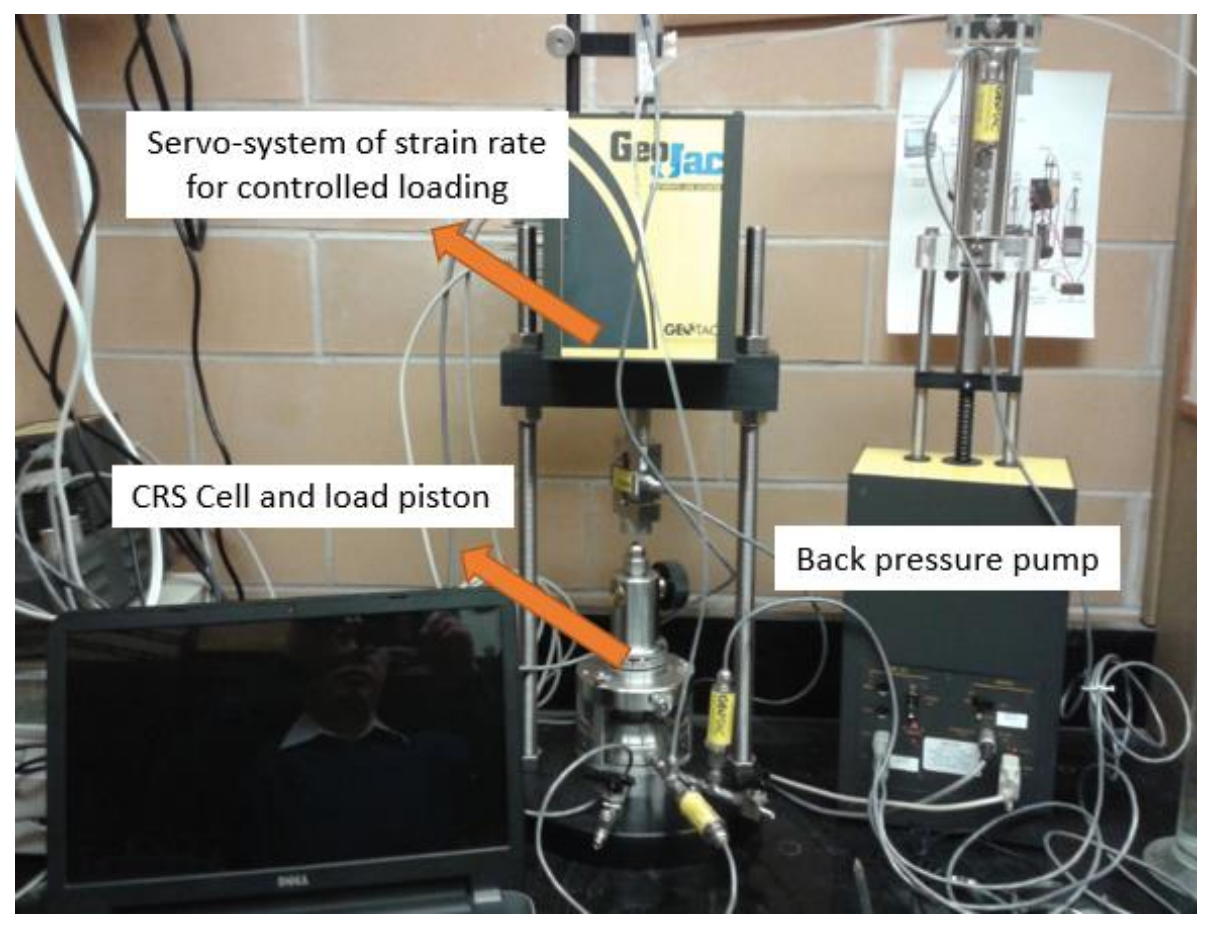

Figure 4.7 CRS test system employed at Ryerson University 


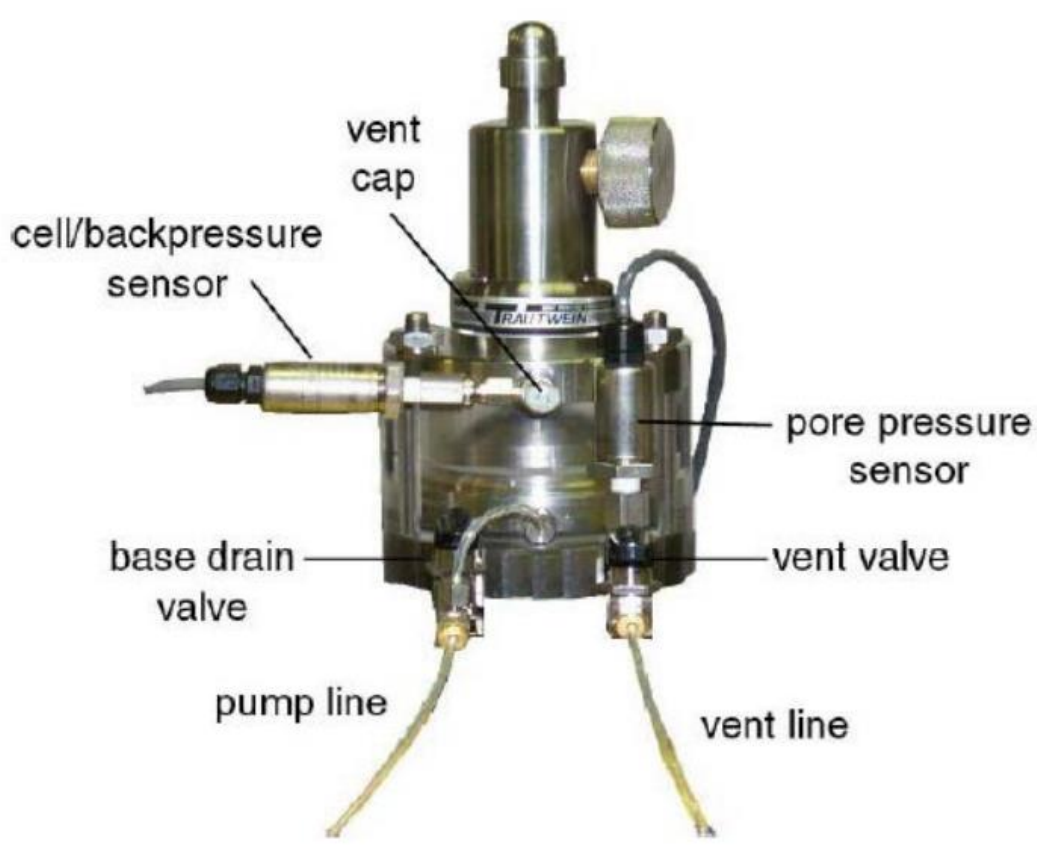

Figure 4.8 Connection of consolidation cell used at Ryerson University (Trautwein, 2001)

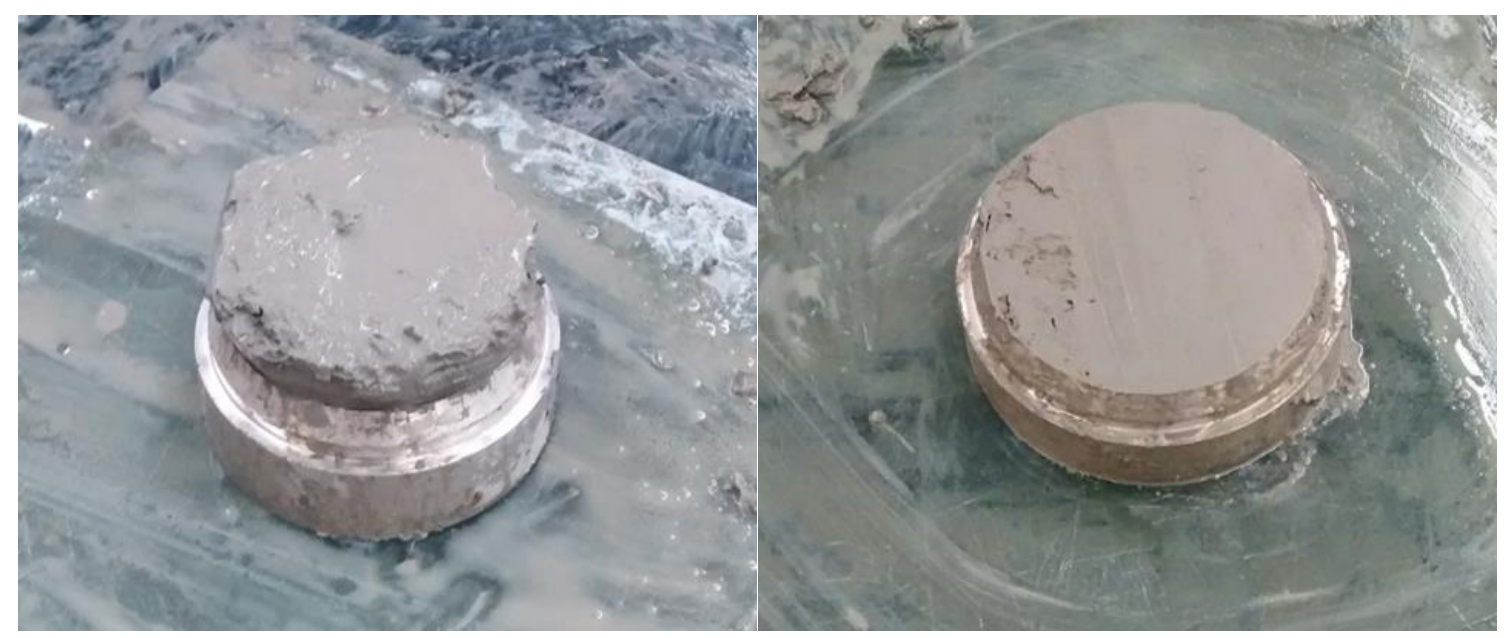

Figure 4.9 Trimming of Champlain Sea clay samples for CRS tests 


\subsection{NUMERICAL SIMULATION OF CRS TESTS ON CHAMPLAIN SEA CLAY}

\subsubsection{OVERVIEW OF CRS TEST RESULTS ON CHAMPLAIN SEA CLAY}

The tested marine clay has the void ratio ranging from 1.37 to 2.44 . Normalizing effective stresses $\sigma^{\prime}$ with $\sigma_{c}^{\prime}$ yields more comparable results of various depths plotted in Figure 4.10. $\sigma_{c}^{\prime}$ is the preconsolidation pressure.

The figure explicitly shows that Champlain Sea clay possesses an "S-shaped" relationship. Compression curves are featured by collapsed post-yield segments accompanied by tremendous volumetric strains. Additionally, it is observed that the samples retrieved close to the ground surface and in deeper area, such as $4.0 \mathrm{~m}, 4.6 \mathrm{~m}$, and $34.1 \mathrm{~m}$, appear to be less compressible than the middle layers, such as $8.5 \mathrm{~m}$ and $30.4 \mathrm{~m}$.

Vertical permeability $k$ has been widely observed to vary with void ratios. It is a common practice to introduce the permeability change index $C_{k}$ to describe this dependence. An empirical correlation of $C_{k}=0.5 \sim 0.55 e_{0}$ has been reported (Leroueil et al. 1990, Shi and Salloum 2018). However, it is also interesting to see that the Champlain Sea clay in this study exhibits an explicit $\Delta \varepsilon_{\mathrm{v}} \sim \Delta \log k$ dependence, as seen in Figure 4.11. 


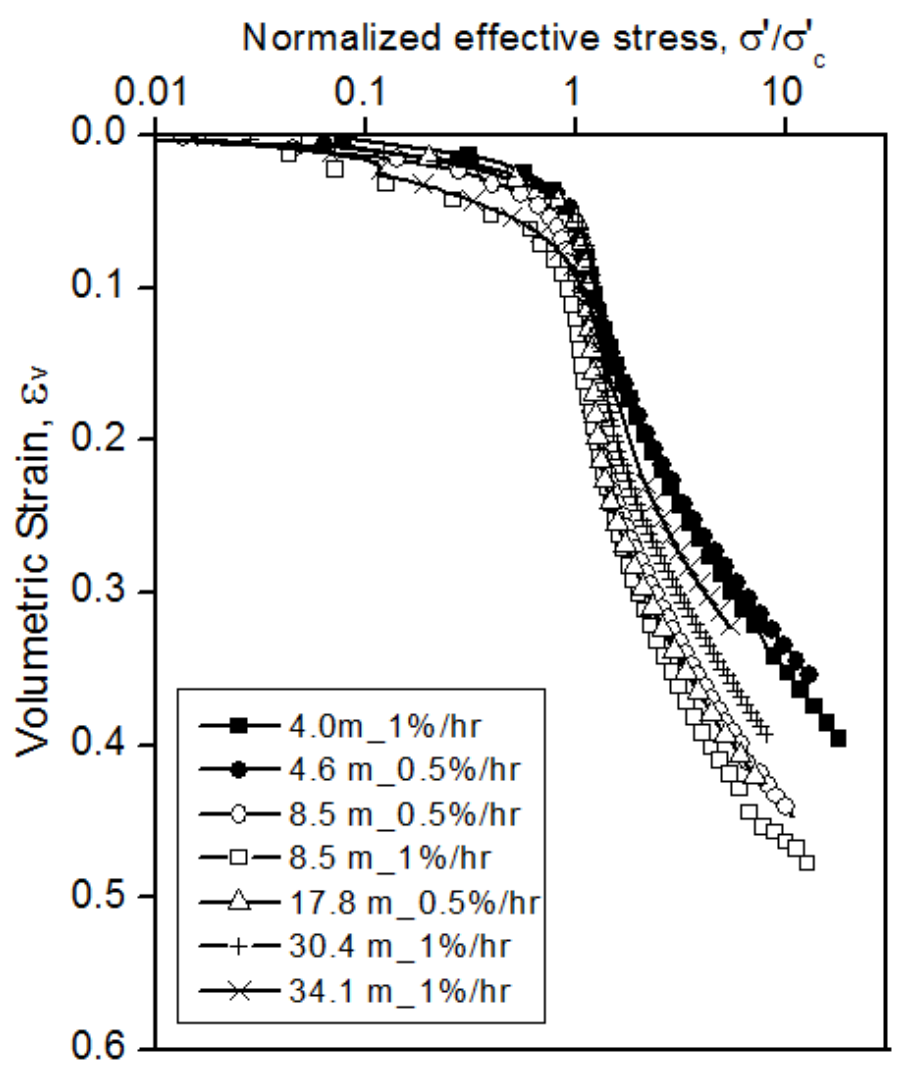

Figure $4.10 \varepsilon_{\mathrm{v}} \sim \log \left(\sigma^{\prime} / \sigma_{\mathrm{c}}^{\prime}\right)$ of the tested Champlain Sea Clay

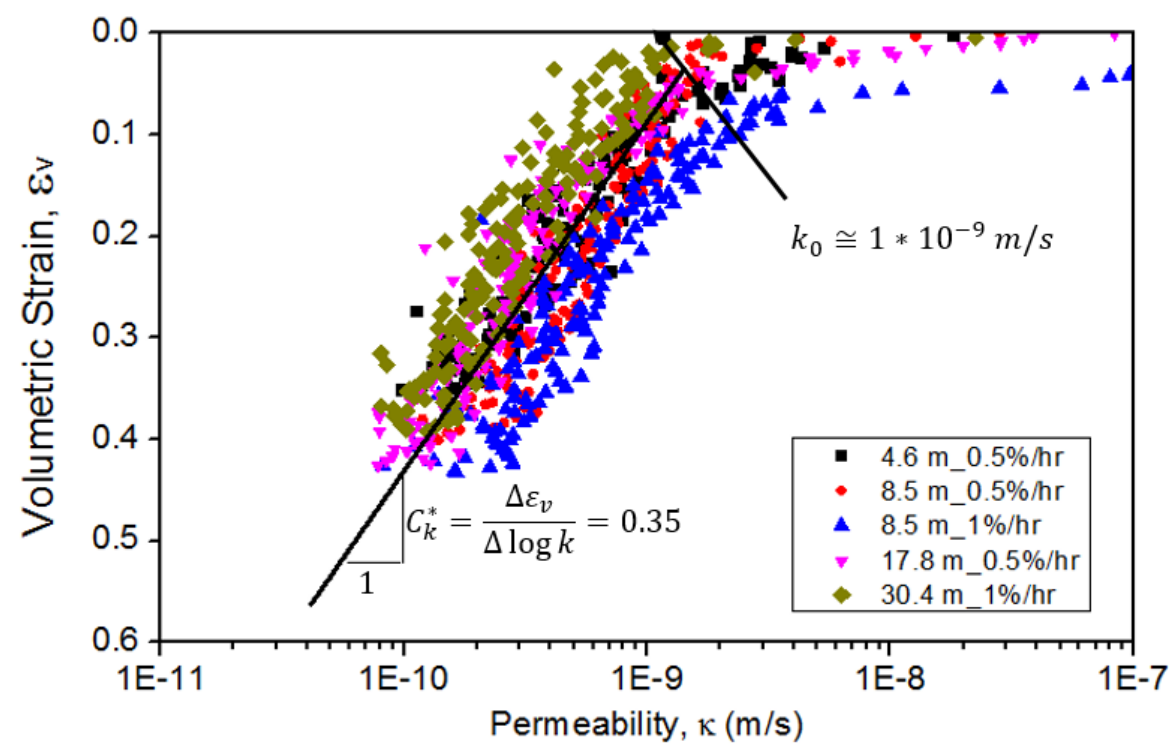

Figure $4.11 \varepsilon_{\mathrm{v}} \sim \mathrm{k}$ dependence of the tested Champlain Sea clay 
The decrease of permeability can be then represented by introducing the modified permeability change index $C_{k}^{*}$ :

$$
C_{k}^{*}=\frac{C_{k}}{1+e_{0}}=\frac{\Delta \varepsilon_{\mathrm{v}}}{\Delta \log k}=0.35
$$

where $C_{k}^{*}$ is the modified version of $C_{k}$ defined as

$$
C_{k}=\frac{\Delta e}{\Delta \log k}
$$

It then follows the estimation of the variation between $C_{k}$ and $e_{0}$ by

$$
C_{k}=0.35\left(1+e_{0}\right)
$$

\subsubsection{MODEL DESCRIPTION FOR CRS TEST SIMULATION}

The circular CRS sample is modeled as an axisymmetric problem in 2D Plaxis software (Plaxis V8.2). A half model is established with a diameter of $3.175 \mathrm{~cm}$ and a height of $2.54 \mathrm{~cm}$ to be consistent with test conditions. The left and right boundaries are fixed for horizontal displacements, whereas the bottom boundary fixes for both vertical and horizontal displacements. Drainage is only allowed at the top surface where the displacement loading is imposed. A porous stone is placed on the top for the consolidation drainage, while other boundaries are kept undrained. Figure 4.12 has more details.

To ensure sufficient accuracy of model predictions, "fine" global coarseness has been chosen to discretize the model into more than 200 triangular elements. A typical simulation takes about 10 min to complete. A point at the bottom left corner has been used for tracking axial strains, excess pore pressures and effectives stresses. However, it should be noted that the average effective stress is used for the result presented in this chapter. The calculation of the average effective stress follows the suggestion of Equation [4.9]. 

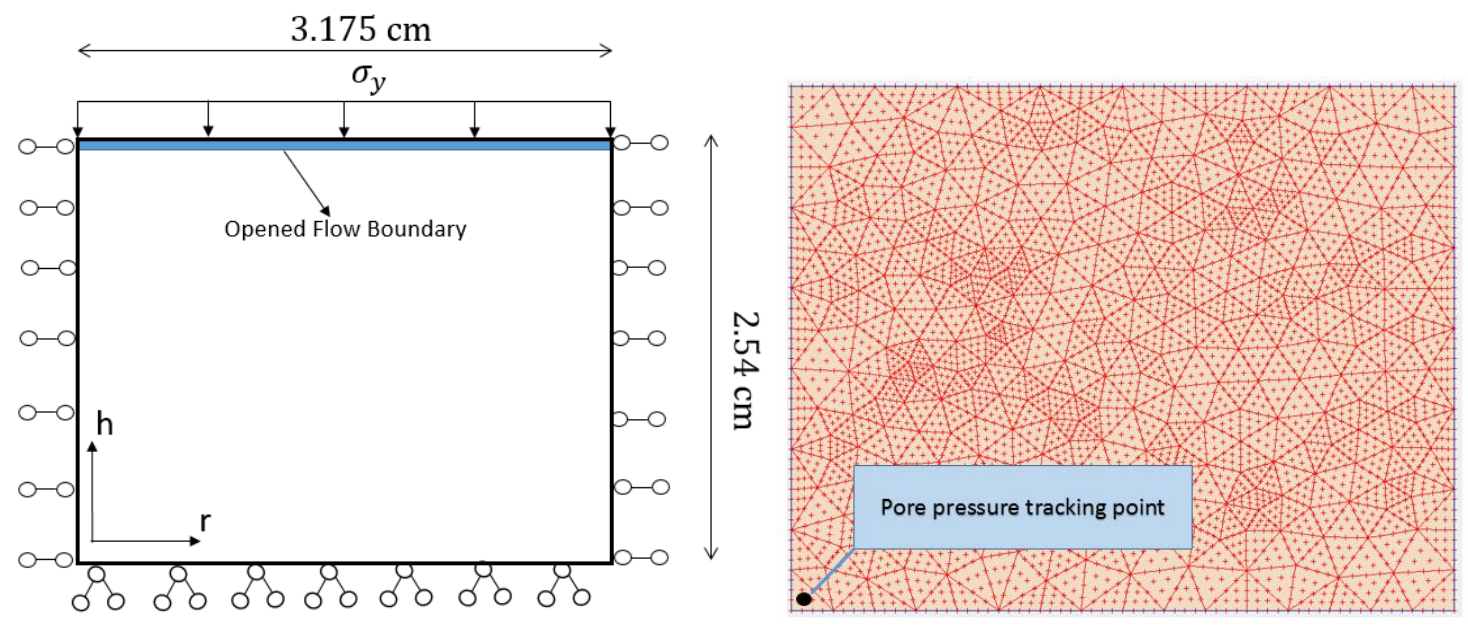

Figure 4.12 Plaxis axisymmetric geometry for CRS test modeling

\subsubsection{PARAMETERS INPUT FOR CRS TEST SIMULATION}

Material models used for the CRS test simulation include MEVP-DS, MEVP, S-CLAY1S, SCLAY1, and SSC model. This attempt is to investigate the contribution of different soil features, such as deviatoric softening, creep, destructuration, and anisotropy, to the model prediction. Note that S-CLAY1S and S-CLAY1 are reduced cases of MEVP model. Table 4.1 specifies details of these models.

In addition, SSC is an isotropic EVP model employing the MCC volumetric hardening rule to describe its cap surface hardening. Destructuration is not considered in SSC, and the model assumes a Mohr-Coulomb failure envelop. SSC is a commercial model embedded in the Plaxis software. Readers are suggested refer to (Plaxis 2002) for more details.

Table 4.1 Soil models used for CRS tests modeling

\begin{tabular}{lll}
\hline Case Name & \multicolumn{1}{c}{ Details } & Comments \\
\hline MEVP-DS & Considers deviatoric softening, viscosity, destructuration and anisotropy & The modified MEVP \\
MEVP & Considers EVP, destructuration and anisotropy & MEVP \\
S-CLAY1S & Considers destructuration and anisotropy & Reduced version of MEVP \\
S-CLAY1 & Considers only anisotropy & Reduced version of MEVP \\
SSC & An isotropic commercial EVP model embedded in Plaxis & \\
\hline
\end{tabular}




\section{To clarify the difference between $\lambda, \lambda_{i}$, and $\lambda_{0}$}

It can be noticed that there are three compression indices used in the dissertation, which can be confusing if not understood properly. The clarification of the difference can be seen in Table 4.2.

First of all, $\lambda$ is the conventional compression index for S-CLAY1 and SSC that cannot consider destructuration. For both models, an average $\lambda$ of the whole loading range is used. As such, the range of $\lambda$ could be typically from $1 \sigma_{c}^{\prime}$ to $10 \sigma_{c}^{\prime}$. It can be up to $15 \sigma_{c}^{\prime}$ depending on the maximum vertical stress tests reached, which can be approximately $2 \mathrm{MPa}$. The practice of using an averaged $\lambda$ for S-CLAY1 and SSC is to obtain more comparable excess pore pressure predictions of other models. The reason is that excess pore pressures are found to be highly dependent on volumetric strains.

$\lambda_{i}$ is the intrinsic compression index estimated from the linear segment of the compression curve at high stress levels. At this stage, soil structures are considered totally removed. This parameter will be assigned to MEVP and S-CLAY1S model that consider destructuration, but not deviatoric softening. The determination of $\lambda_{i}$ is articulated in (Koskinen et al. 2002).

$\lambda_{0}$ is for MEVP-DS model and is estimated using the correlation $\lambda_{0}=\frac{\lambda_{i}}{e^{\frac{1.21}{M} * 0.5}}$. The correlation is based on the assumption that the $\eta$ applied during CRS tests is close to the 1-D case of 0.5 .

Table 4.2 Clarification of different $\lambda$ used for modeling

\begin{tabular}{cll}
\hline & Models related to this parameter & Determination of this parameter \\
\hline$\lambda$ & S-CLAY1, SSC & $\begin{array}{l}\text { The averaged } \lambda \text { of the compression curve, } \\
\text { ranging from } 5 \text { to } 15 \sigma_{c}^{\prime} \text { depending on } \\
\text { individual tests }\end{array}$ \\
\hline$\lambda_{\boldsymbol{i}}$ & MEVP, S-CLAY1S & $\begin{array}{l}\text { The intrinsic compression index estimated from } \\
\text { the linear segment at high stress level }\end{array}$ \\
\hline$\lambda_{\mathbf{0}}$ & MEVP-DS model & Estimated from $\lambda_{0}=\frac{\lambda_{i}}{e^{\frac{1.21}{M} * 0.5}}$ \\
\hline
\end{tabular}

\section{Determination of $e_{0}, \gamma, M, \psi$ and $\sigma_{c}^{\prime}$ for all models}

Parameters input for MEVP-DS and other simplified models are summarized in Table 4.3 and Table 4.4. In addition, Table 4.4 also presents the parameters input for SSC model. The initial 
void ratio $e_{0}$ and the unit weight $\gamma$ of each sample were determined from density tests and water content tests. Values for $M$ were determined from a series of CIU tests performed on undisturbed Champlain Sea clay samples, and more details will be presented in Chapter 5. The secondary consolidation index $\psi$ is specified from 1-D creep tests. $\sigma_{c}^{\prime}$ are input to fit the pre-consolidation pressures identified using bilogarithmic methods (Kirstein 2017). Due to the strain rate effect, the input $\sigma_{c}^{\prime}$ can be around $15 \%$ to $20 \%$ lower than the identified values.

\section{Determination of destructuration parameters $\kappa, \lambda_{i}, \chi_{0}, \xi$, and $\xi_{d}$ for MEVP model}

Destructuration parameters were calibrated to fit the tested compression curves. Figure 4.13 presents how destructuration parameters $\lambda_{i}$ and $\chi_{0}$ of MEVP cases are interpreted, using 17.8 $\mathrm{m} \_0.5 \%$ test result as an example. $\kappa$ is obtained from the recompression segment. $\xi$ also needs to be calibrated by trial simulations. Note that $\xi_{d}$ is a less sensitive parameter and a recommended $\xi_{d}=0.2$ is taken for analysis (Yin et al. 2011).

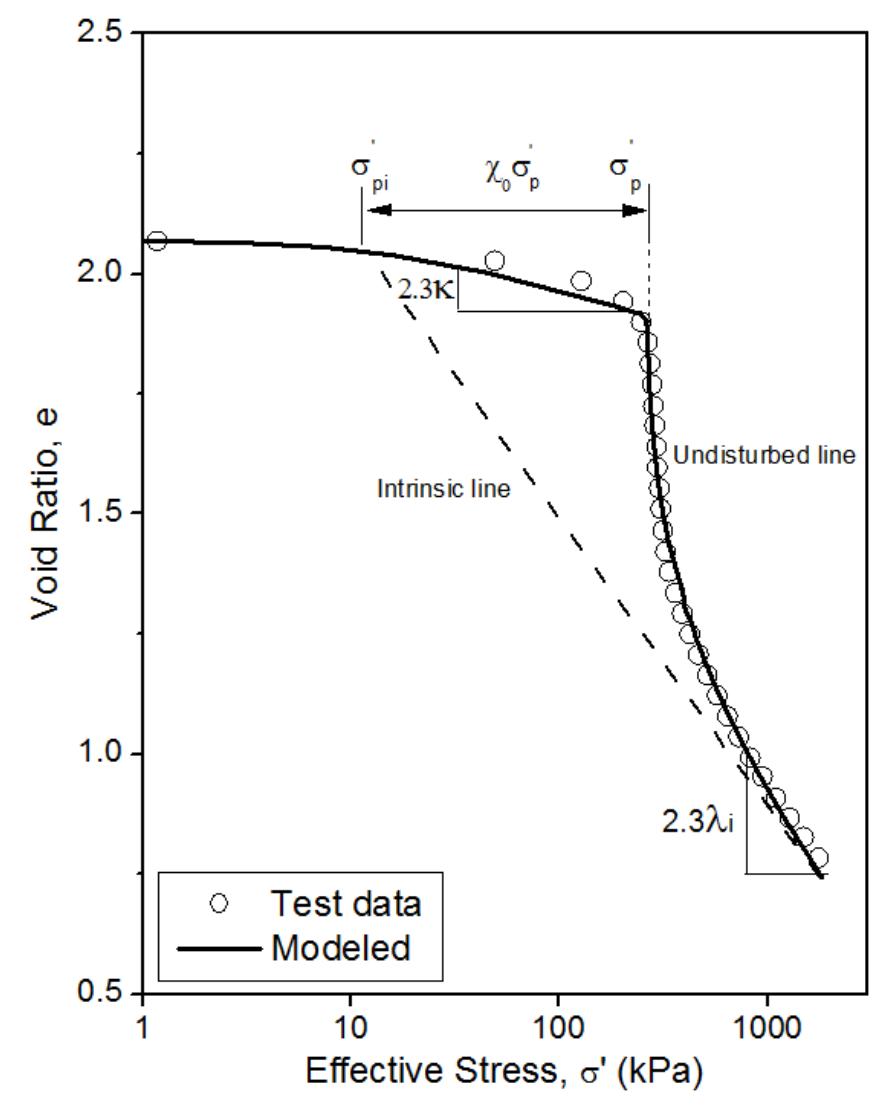

Figure 4.13 Estimation of $\kappa$ and $\lambda_{\mathrm{i}}$ from CRS test on Champlain Sea Clay

Determination of $\varsigma, \lambda_{0}, \chi_{0}, \xi$, and $\xi_{d}$ for MEVP-DS model 
However, $\chi_{0}$ and $\xi$ values input for MEVP-DS model are distinct from MEVP cases, in that both destructuration and deviatoric softening are proposed in MEVP-DS to describe the yield surface contraction. The difference between destructuration and deviatoric softening is that destructuration represents structured surface contraction, while deviatoric softening for intrinsic surface contraction. As such, independent calibrations should be done to calibrate destructuration parameters, along with $\varsigma$ input for MEVP-DS model. The sensitivity of these parameters will be investigated later.

\section{Determination of anisotropy parameters $\alpha_{0}, \omega, \omega_{d}$ for MEVP-DS and MEVP}

Three anisotropic parameters for both MEVP and MEVP-DS, including $\alpha_{0}, \mu$ and $\beta$, are estimated following the procedures stated in Wheeler et al. (2003). The initial inclination of yield surface $\alpha_{0}$ is determined by

$$
\alpha_{0}=\frac{\eta_{K 0}^{2}+3 \eta_{K 0}-M^{2}}{3}
$$

where $\eta_{K 0}$ is the stress ratio of $K_{0}$ state, which is the function of the friction angle $\varphi$

$$
\eta_{K 0}=\frac{3 \sin \varphi^{\prime}}{3-2 \sin \varphi^{\prime}}
$$

The relative rate of yield surface rotation $\omega$ can be estimated by

$$
\omega=\frac{3\left(4 M^{2}-4 \eta_{K 0}^{2}-3 \eta_{K 0}\right)}{8\left(\eta_{K 0}^{2}-M^{2}+2 \eta_{K 0}\right)}
$$

The determination of the absolute rate of yield surface rotation $\omega_{d}$ is based on

$$
\omega_{d}=\frac{1+e_{0}}{\lambda} \ln \frac{10 M^{2}-2 \alpha_{0} \omega}{M^{2}-2 \alpha_{0} \omega}
$$


Table 4.3 Initial values of the parameters for MEVP-DS model used in the simulation

\begin{tabular}{|c|c|c|c|c|c|c|c|c|c|c|c|c|c|c|c|c|c|c|}
\hline Depth & $\begin{array}{c}\boldsymbol{\gamma} \\
\mathrm{kN} / \mathrm{m}^{3}\end{array}$ & $\begin{array}{c}\boldsymbol{\sigma}_{\boldsymbol{c}}^{\prime} \\
\mathrm{kPa}\end{array}$ & $\begin{array}{c}\boldsymbol{k}_{\boldsymbol{o}} \\
\times 10^{-9} \mathrm{~m} / \mathrm{s}\end{array}$ & $C_{k}$ & $\begin{array}{r}M \\
-\end{array}$ & $\begin{array}{c}K_{0} \\
-\end{array}$ & $\begin{array}{l}\boldsymbol{v} \\
-\end{array}$ & $\psi$ & $\begin{array}{c}e_{0} \\
-\end{array}$ & $\begin{array}{c}\lambda_{0} \\
-\end{array}$ & $\begin{array}{l}S \\
-\end{array}$ & $\begin{array}{l}\boldsymbol{\kappa} \\
-\end{array}$ & $\begin{array}{c}\chi_{0} \\
-\end{array}$ & $\begin{array}{l}\xi \\
-\end{array}$ & $\begin{array}{c}\xi_{d} \\
-\end{array}$ & $\begin{array}{c}\alpha_{0} \\
-\end{array}$ & $\begin{array}{c}\omega \\
-\end{array}$ & $\begin{array}{c}\omega_{d} \\
-\end{array}$ \\
\hline 4 & 16 & 112 & 1.0 & 1.1 & 1.33 & 0.7 & 0.2 & 0.018 & 2.02 & 0.2 & 3 & 0.025 & 10 & 10 & 0.2 & 0.5 & 20 & 0.89 \\
\hline 4.6 & 16 & 112 & 1.0 & 1.1 & 1.33 & 0.7 & 0.2 & 0.018 & 2.02 & 0.23 & 3.5 & 0.025 & 11 & 11 & 0.2 & 0.5 & 20 & 0.89 \\
\hline 8.5 & 16 & 120 & 1.0 & 1.2 & 1.33 & 0.7 & 0.2 & 0.022 & 2.44 & 0.3 & 4 & 0.035 & 11 & 11 & 0.2 & 0.5 & 20 & 0.89 \\
\hline 17.8 & 16 & 200 & 1.0 & 1.1 & 1.33 & 0.7 & 0.2 & 0.022 & 2.07 & 0.25 & 3.5 & 0.025 & 12 & 12 & 0.2 & 0.5 & 20 & 0.89 \\
\hline 30.4 & 19 & 280 & 1.0 & 1.0 & 1.33 & 0.7 & 0.2 & 0.018 & 1.86 & 0.26 & 3 & 0.025 & 10 & 10 & 0.2 & 0.5 & 20 & 0.89 \\
\hline 34.1 & 19 & 320 & 1.0 & 0.8 & 1.30 & 0.7 & 0.2 & 0.016 & 1.37 & 0.18 & 2.5 & 0.020 & 8 & 8 & 0.2 & 0.5 & 20 & 0.89 \\
\hline
\end{tabular}

Note: the bolded numbers are to highlight the difference in parameter input between MEVP-DS, MEVP family, and SSC model.

Table 4.4 Initial values of the parameters for MEVP, S-CLAY1S, S-CLAY1, and SCC used in the simulation

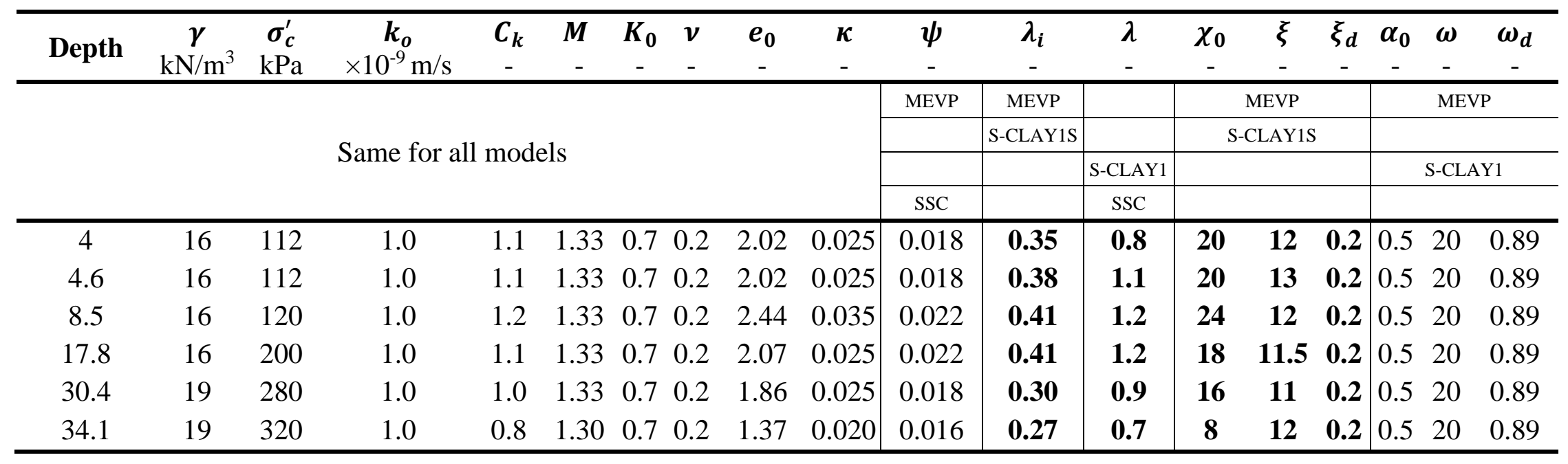




\subsubsection{MODEL SIMULATION OF $\sigma^{\prime}$ DURING CRS TESTS}

Figure 4.14 is presented for the simulated $\varepsilon_{\mathrm{v}} \sim \log \sigma^{\prime}$ relationships for four samples. Two samples are selected from shallow depths $(4.6 \mathrm{~m}$ and $8.5 \mathrm{~m})$, other two are from deeper areas (30.4 $\mathrm{m}$ and $34.1 \mathrm{~m})$

The model predictions demonstrate that MEVP-DS, MEVP, and S-CLAY1S capture the compression curves more precisely than S-CLAY1 and SSC. Therefore, softening behavior is the dominant factor for structured clay modeling. Viscosity is a less important factor here as the difference between MEVP and S-CLAY1S is marginal. Anisotropy influence is not significant either as S-CLAY1 and SSC predictions are similar.
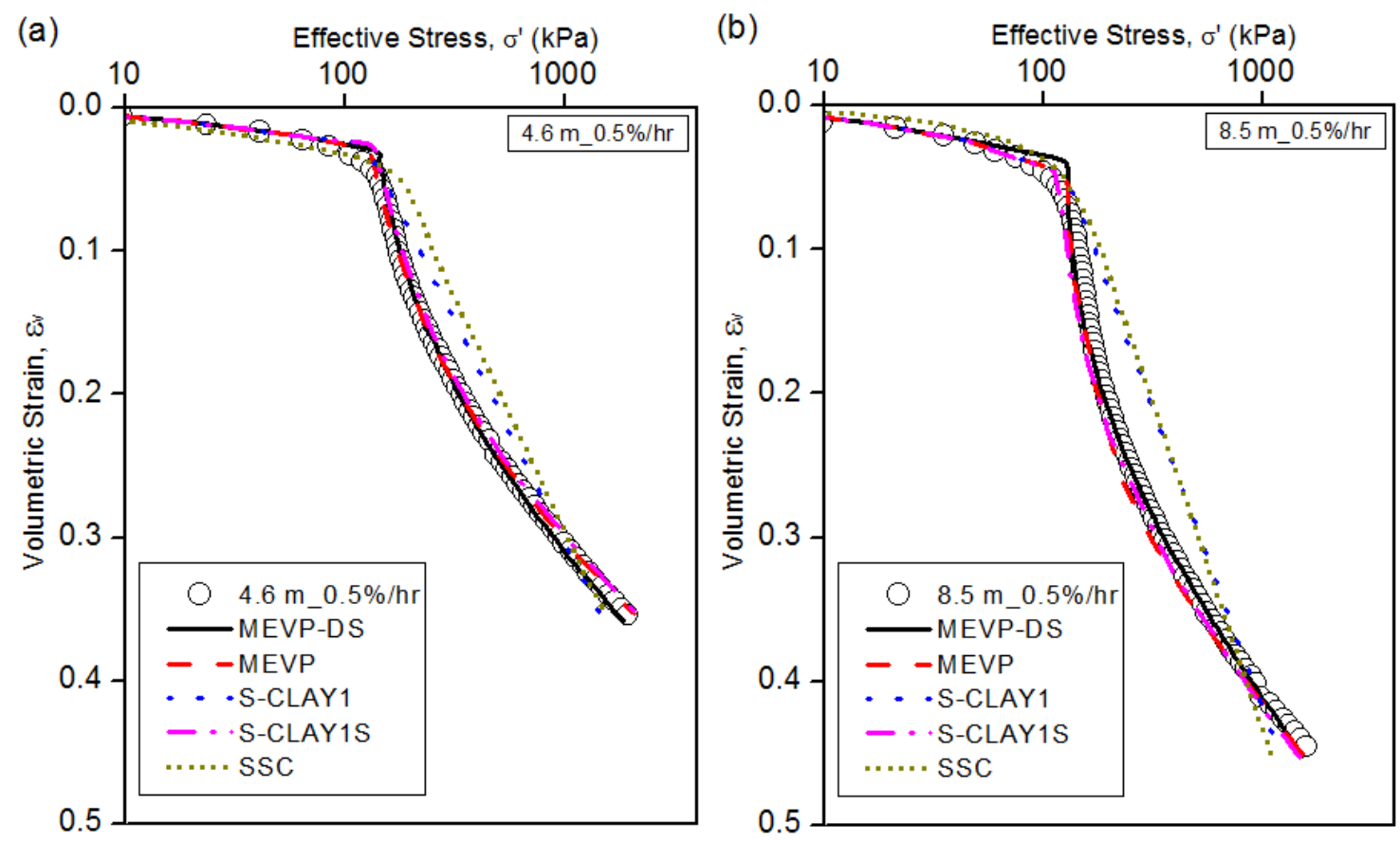

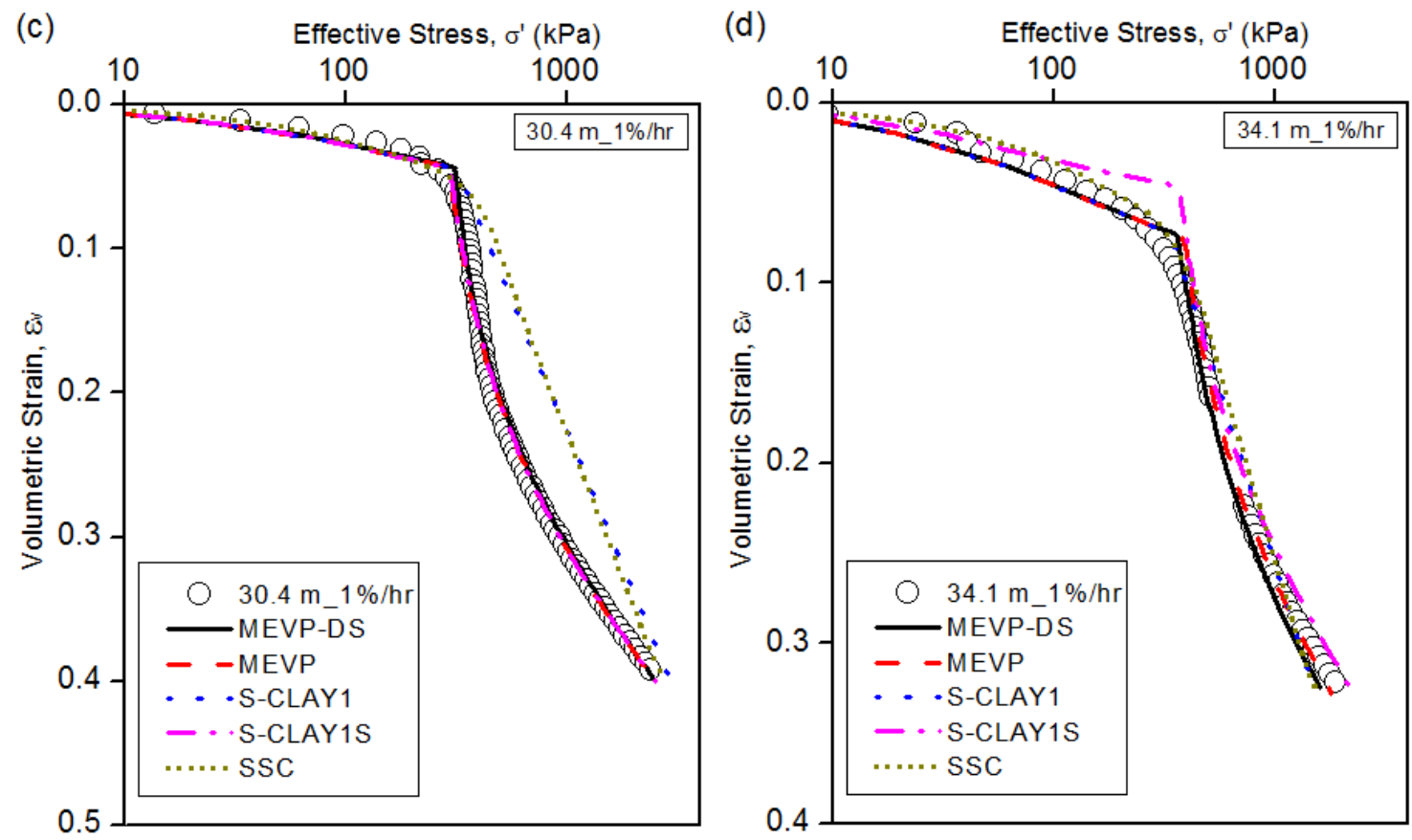

Figure 4.14 Simulation of CRS tests of Champlain Sea clay

\subsubsection{MODEL SIMULATION OF $\triangle \boldsymbol{u}_{b}$ DURING CRS TESTS}

Figure 4.15 compares the excess pore pressure generated in eight CRS tests, using three types of expressions, $\Delta \mathrm{u}_{\mathrm{b}}$, the hydraulic gradient $\mathrm{i}$, and $\mathrm{R}=\Delta \mathrm{u}_{\mathrm{b}} / \sigma^{\prime} . \mathrm{i}$ is defined as follows

$$
i=\frac{\Delta u_{b}}{\gamma_{w} h_{o}(1-\varepsilon)}
$$

The hydraulic gradient can be an indicative of Darcy and non-Darcy flow condition. However, the boundary value of $\mathrm{i}$ has yet been agreed by the reviewed literatures. The suggested gradient boundary where Darcy flow transfers to non-Darcy flow varies from 13 to 100 (Larsson and Sallfors 1985, Sheahan and Watters 1997, Ahmadi et al. 2011, ASTM 2015).

Figure 4.15 (a) suggests that $1 \% / \mathrm{hr}$ tests generate $\Delta \mathrm{u}_{\mathrm{b}}$ almost twice of $0.5 \% / \mathrm{hr}$ tests, if one compares the tests performed on similar depths. For example, the pair of $8.5 \mathrm{~m} \_0.5 \% / \mathrm{hr}$ and 8.5 $\mathrm{m} \_1 \% / \mathrm{hr}$, and so does $4.6 \mathrm{~m} \_0.5 \% / \mathrm{hr}$ and $4.0 \mathrm{~m} \_1 \% / \mathrm{hr}$. This observation also applies to the $\varepsilon \sim \mathrm{i}$ dependence shown in Figure 4.15 (b). In addition, Figure 4.15 (c) shows a linear downward trend of R with $\sigma^{\prime} / \sigma_{\mathrm{c}}^{\prime}$. 
The model result of Test $4.0 \mathrm{~m} \_1 \% / \mathrm{hr}$ has been presented in Figure 4.16. The result shows that MEVP-DS predicts $\Delta \mathrm{u}_{\mathrm{b}} \sim \sigma^{\prime}$ slightly better than other models. It is followed by MEVP and SCLAY1S, indicating the importance of considering destructuration. All models predict $\varepsilon \sim i$ well except S-CLAY1, which doesn't capture the non-linear relationship.

Figure 4.17 shows some similar findings from Test $30.48 \mathrm{~m} \_1 \% / \mathrm{hr}$, while the improved prediction by MEVP-DS is more tangible. The result implies that deviatoric softening of intrinsic surface should be considered along with structured surface softening. On the other hand, the predictions provided by S-CLAY1 and SSC appear to be inaccurate.

Figure 4.18 summarizes MEVP-DS and MEVP simulations for three tests performed at $0.5 \% / \mathrm{hr}$ strain rate. Figure 4.19 is shown for $1 \% / \mathrm{hr}$ tests. The improvement by MEVP-DS is demonstrated for most of the tests modeled, except for $34.1 \mathrm{~m} \_1 \% / \mathrm{hr}$. However, this test can be less reliable as some data points are missing in the middle segment. This could be due to computer glitches such as automatic system updates popped out, stopping the test occasionally. 

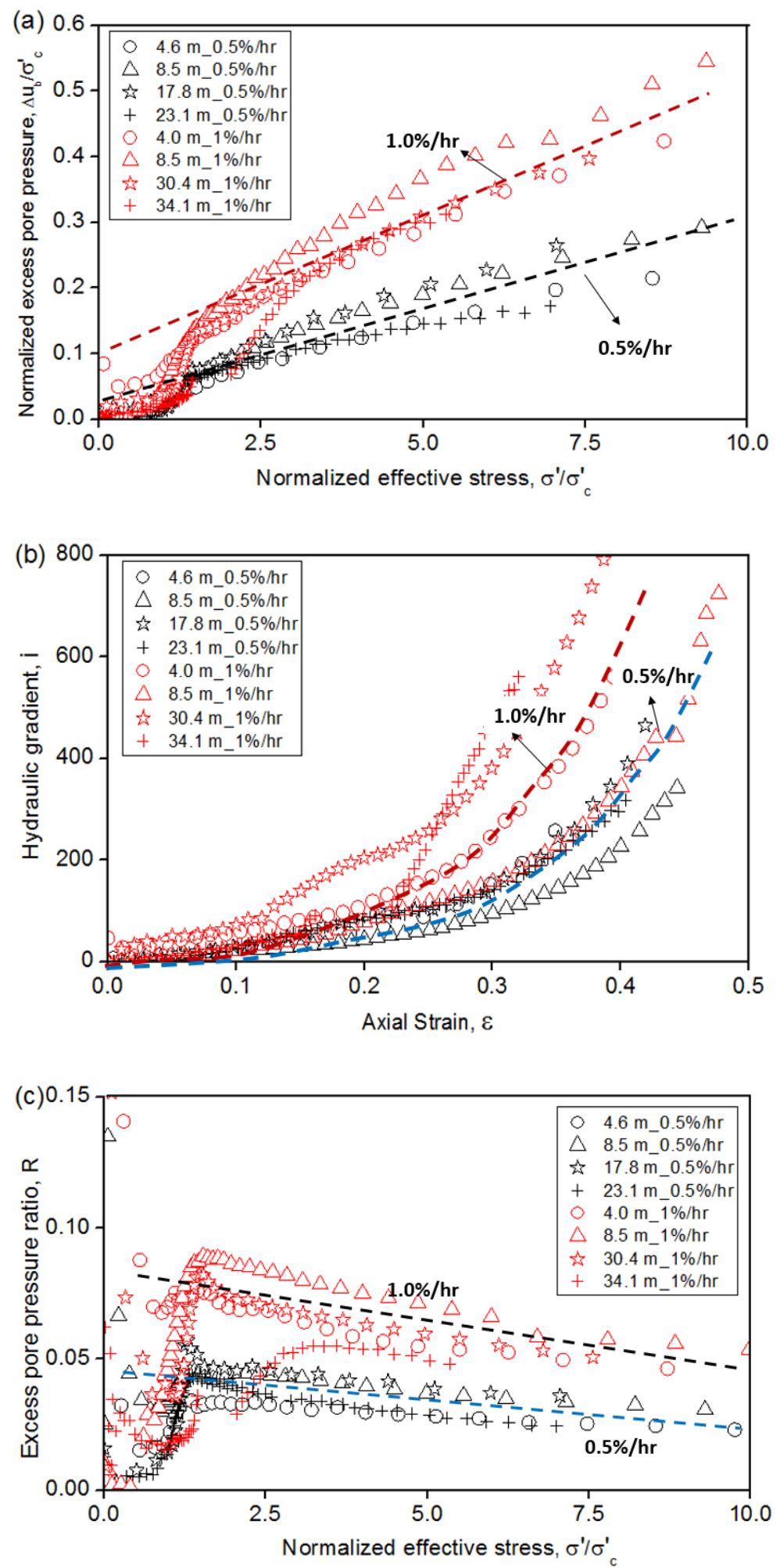

Figure 4.15 Excess pore pressure response of Champlain Sea clays during CRS loading 

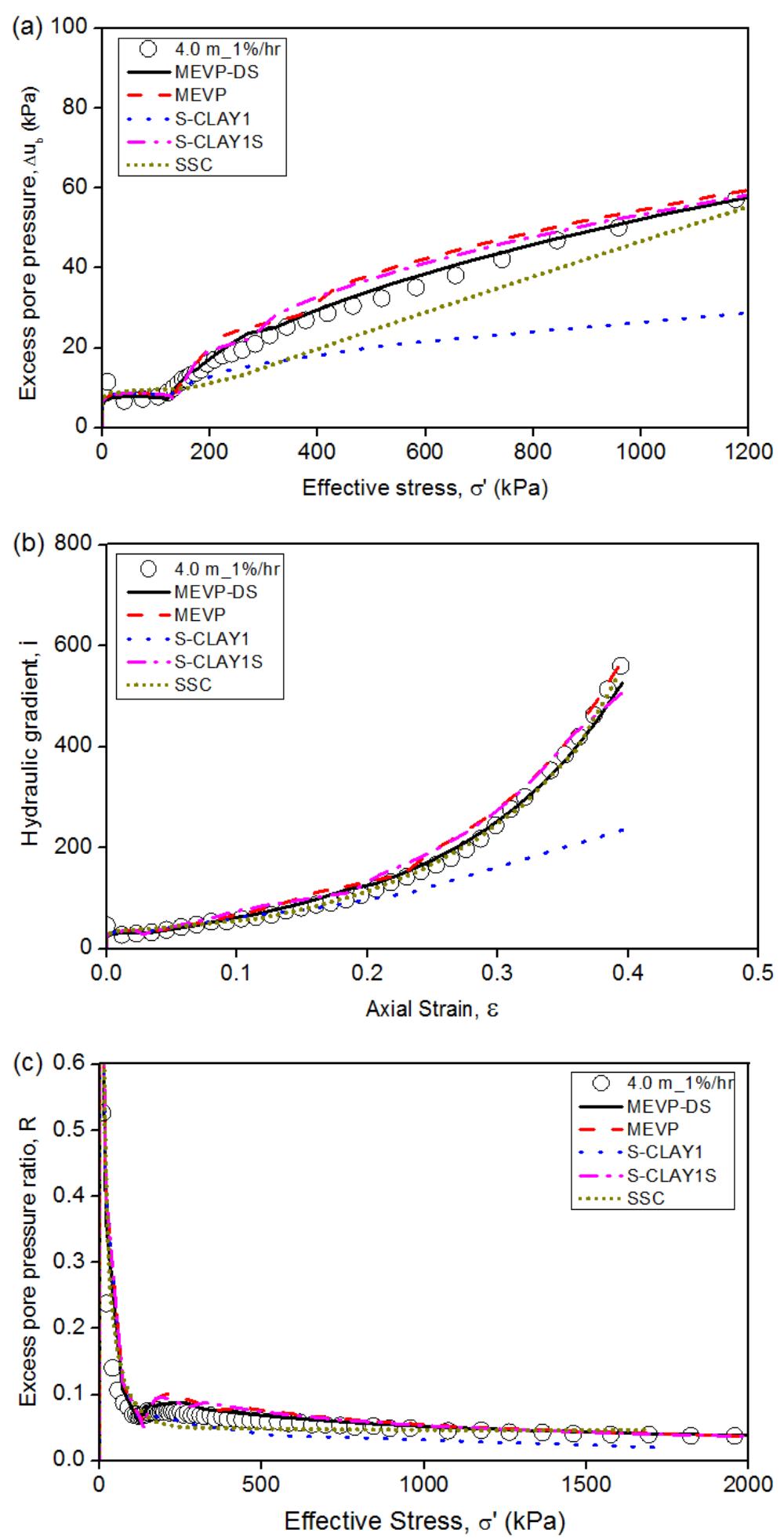

Figure 4.16 Excess pore pressure response of CRS test on $4.0 \mathrm{~m}$ depth sample 

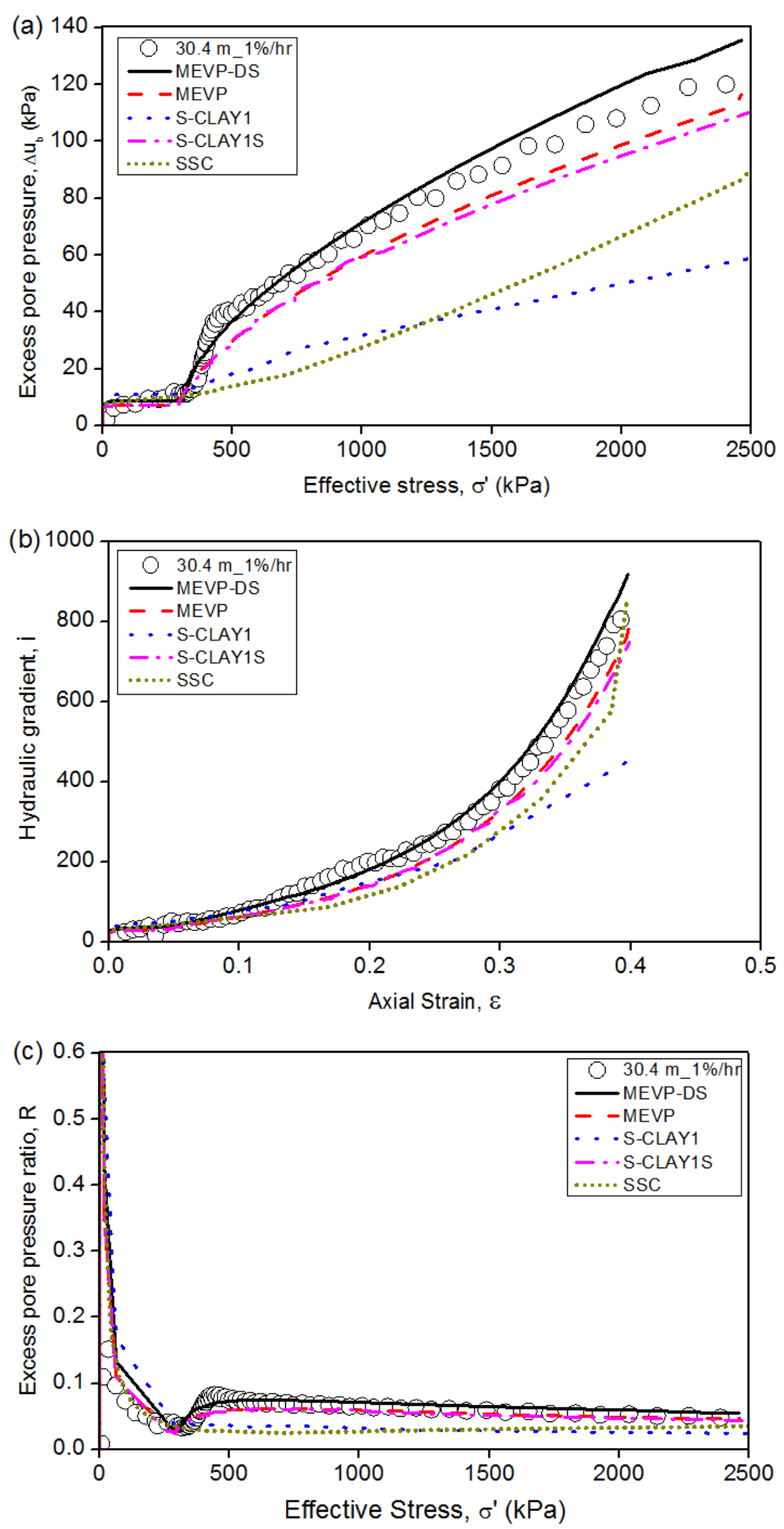

Figure 4.17 Excess pore pressure response of CRS test on $30.4 \mathrm{~m}$ depth sample 

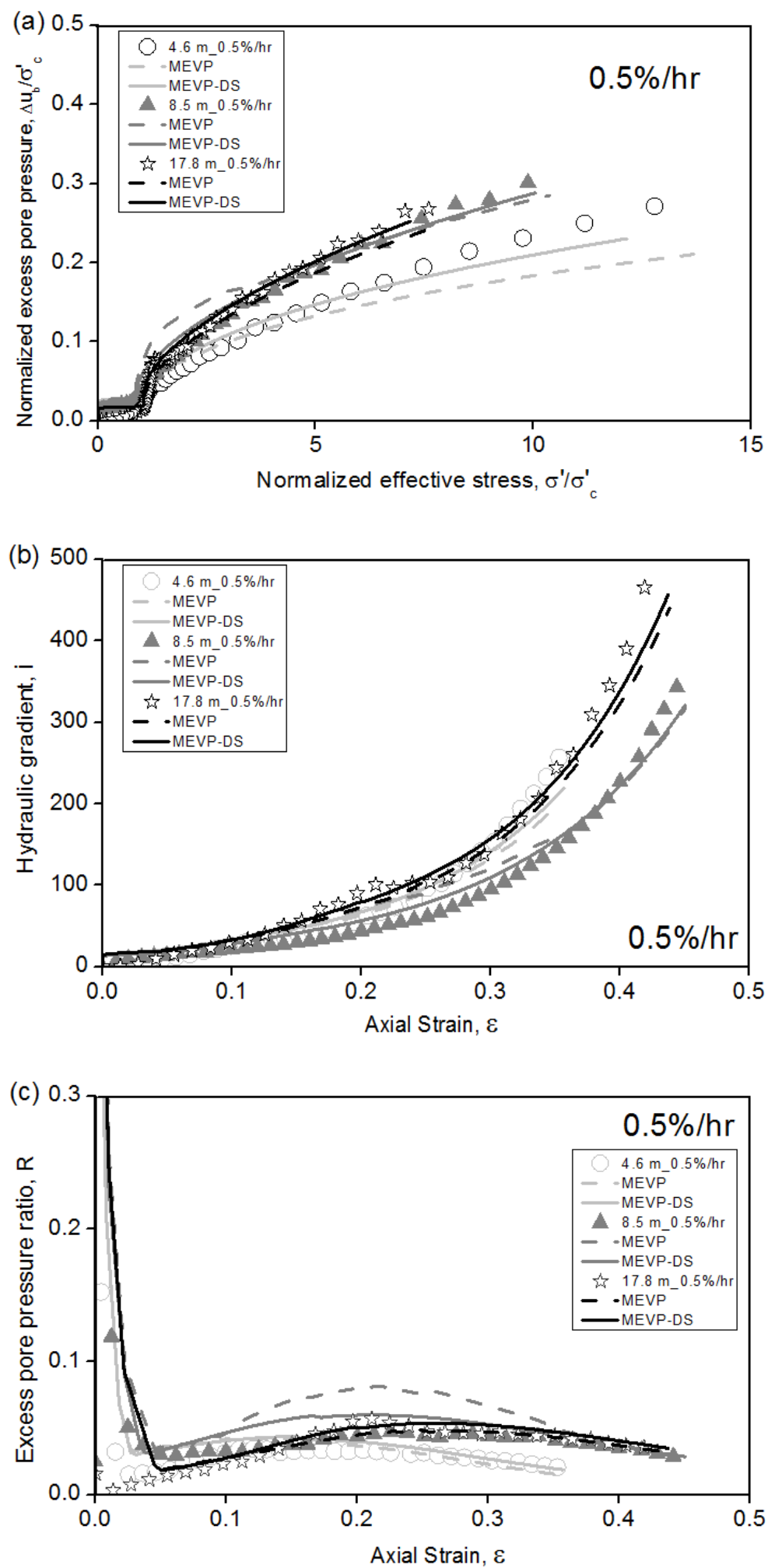

Figure 4.18 Summary of $0.5 \% / \mathrm{hr}$ CRS tests simulation 

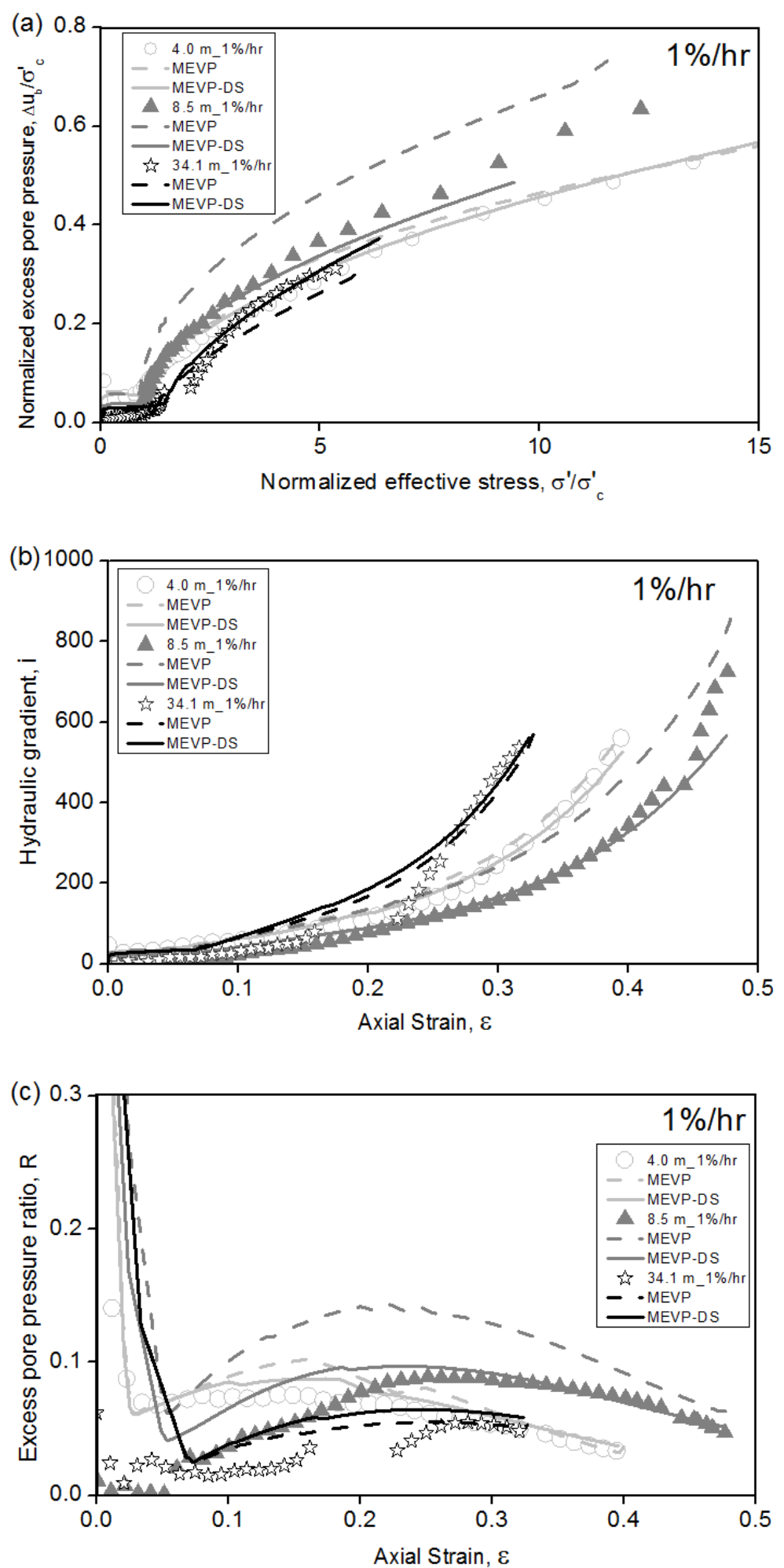

Figure 4.19 Summary of $1.0 \% / \mathrm{hr}$ CRS tests simulation 


\subsection{SENSITIVITY ANALYSIS OF INPUT PARAMETERS OF MEVP-DS MODEL}

A series of CRS test simulations are presented here to study the sensitivity of MEVP-DS parameters. Test $4 \mathrm{~m} \_1 \% / \mathrm{hr}$ is used here as the control test. The reason that this test is chosen is because MEVP-DS model provides good predictions of both effective stress and excess pore pressure responses for this test. Some other tests can also be used, such as $4.6 \mathrm{~m} \_0.5 \% / \mathrm{hr}$ and 30.4 $\mathrm{m} \_1 \% / \mathrm{hr}$.

\subsubsection{COMPARISON OF PARAMETERS SENSITIVITY}

The study is conducted by increasing or reducing all the studied parameters by $15 \%$ to see the impact on the modeling results. The reason for selecting $15 \%$ as the variation is that this value is the maximum variation for $\varsigma$ to fit the $\varepsilon_{\mathrm{v}} \sim \log p^{\prime}$ curve of Test $4 \mathrm{~m} \_1 \% / \mathrm{hr}$. A higher variation, for example, $20 \%$, would result in divergence issues due to stress responses that are too high or too low.

Figure 4.20 indicates that $\varsigma$ and $\lambda_{0}$ are the most sensitive parameters for $\varepsilon_{\mathrm{v}} \sim \log \mathrm{p}^{\prime}$. They are followed by $\alpha_{0}$ and $\chi_{0}$ which exhibit some sensitivity, but not a significant one. Strain rates $(\dot{\varepsilon})$ and $\mathrm{C}_{\mathrm{k}}$ haven't shown much difference at the $15 \%$ variation level.

Figure 4.21 show that $\sigma^{\prime} \sim \Delta \mathrm{u}_{\mathrm{b}}$ is the most sensitive to $\varsigma$ and $\lambda_{0}$. Moreover, $\mathrm{C}_{\mathrm{k}}$ is also an important parameter. Other parameters influence the prediction in a less tangible manner. The summarized prediction of $\varepsilon \sim \mathrm{i}$ demonstrates that $\mathrm{C}_{\mathrm{k}}$ is the most sensitive parameter, shown in Figure 4.22, which is followed by $\dot{\varepsilon}$. Other parameters do not make any noticeable difference.

Figure 4.23 provides a quantified summary on the parameter sensitivity. As $\Delta \mathrm{u}_{\mathrm{b}}$ increases nonlinearly during CRS tests, it would be a reasonable approach to compare parameter influence at a designated effective stress, excess pore pressure, or strain level. As such, the predicted $p^{\prime}$ and i at $40 \%$ volumetric strain, and the $\Delta \mathrm{u}_{\mathrm{b}}$ at $\sigma^{\prime}=1000 \mathrm{kPa}$ are compared to rank parameter sensitivities.

The result indicates that CRS test predictions are most sensitive to $\varsigma, \lambda_{0}$ and $C_{k}$. The reason why $\varsigma$ and $\lambda_{0}$ stay on the top is that both parameters determine the steepness of $\varepsilon_{v} \sim \log p^{\prime}$. Besides, any variations on the logarithmic scale would result in a significant difference if compared in linear 
scales. The same explanation applies to $\mathrm{C}_{\mathrm{k}}$ for excess pore pressure predictions, since this parameter defines the steepness of the $\frac{\Delta e}{\Delta \log k}$ slope.
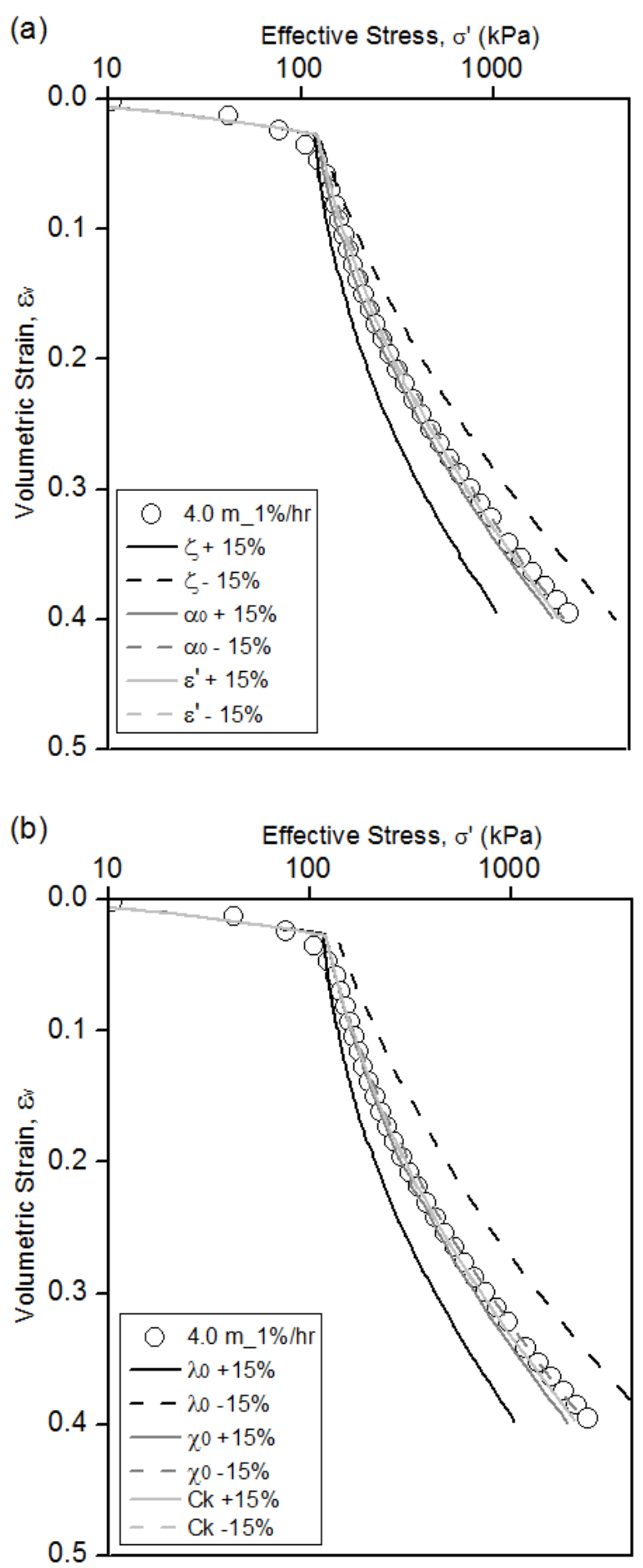
Figure 4.20 Sensitivity of MEVP-DS model parameters in simulating volumetric strain
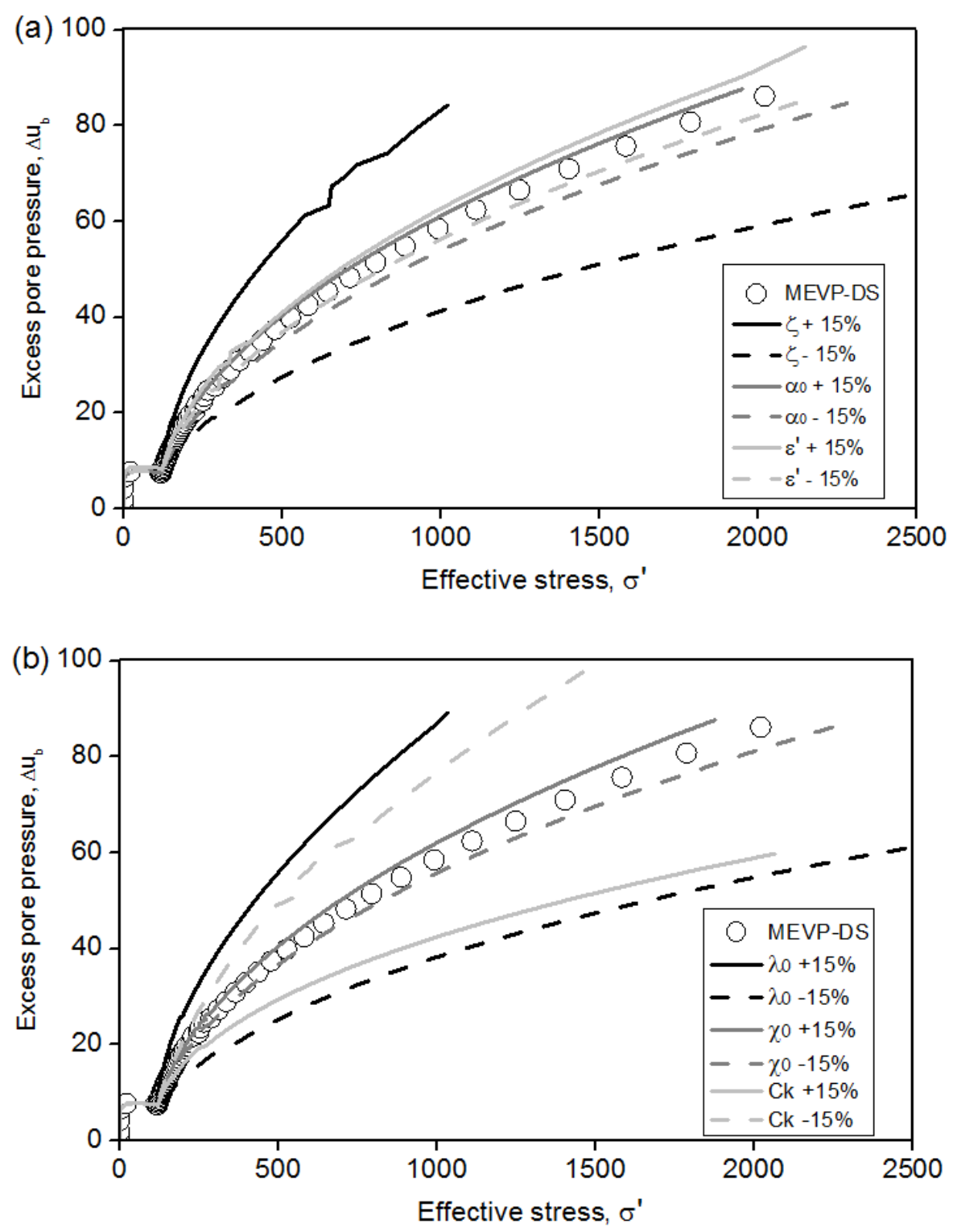

Figure 4.21 Sensitivity of MEVP-DS model parameters in simulating excess pore pressure 

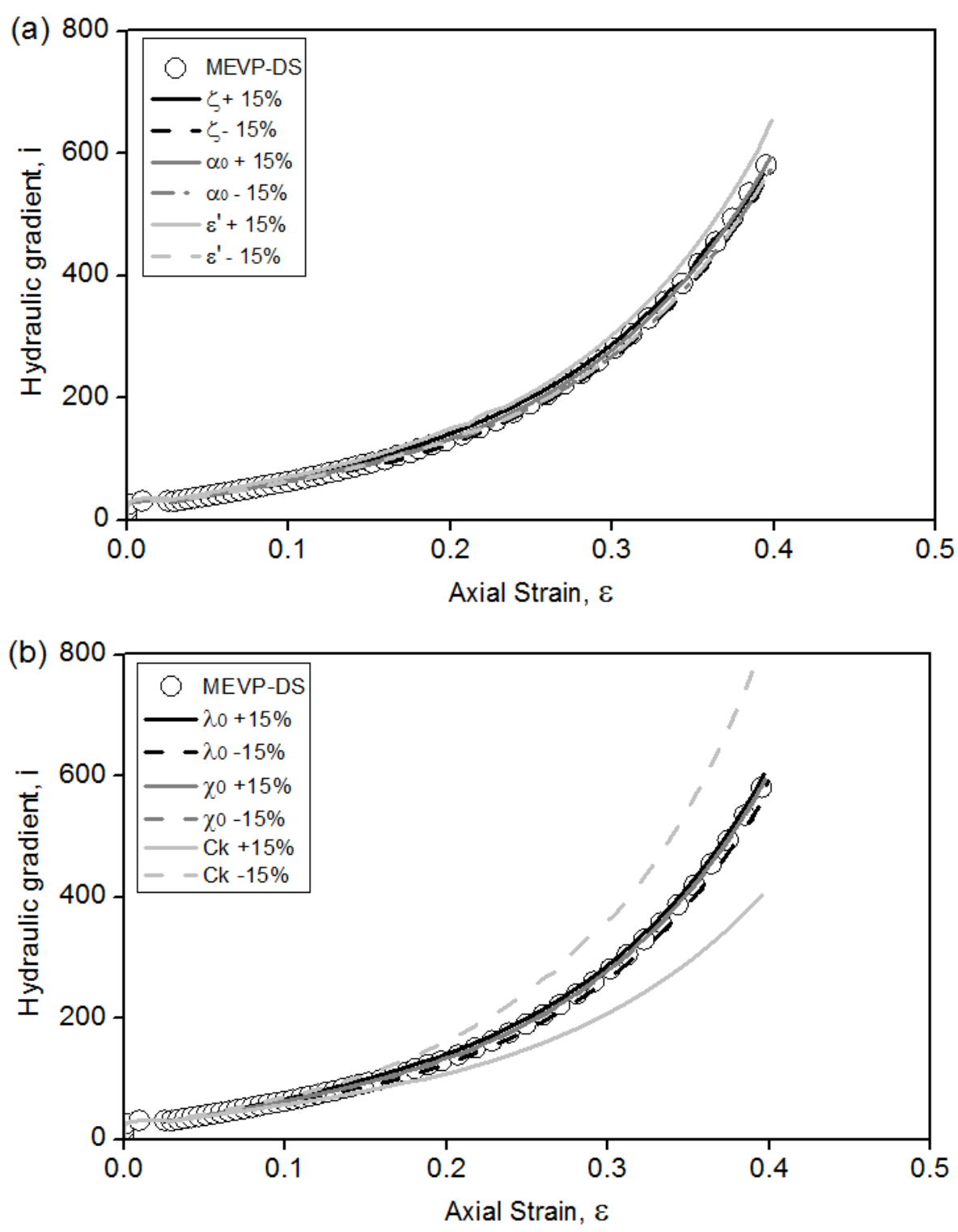

Figure 4.22 Sensitivity of MEVP-DS model parameters in simulating hydraulic gradient 
(a)

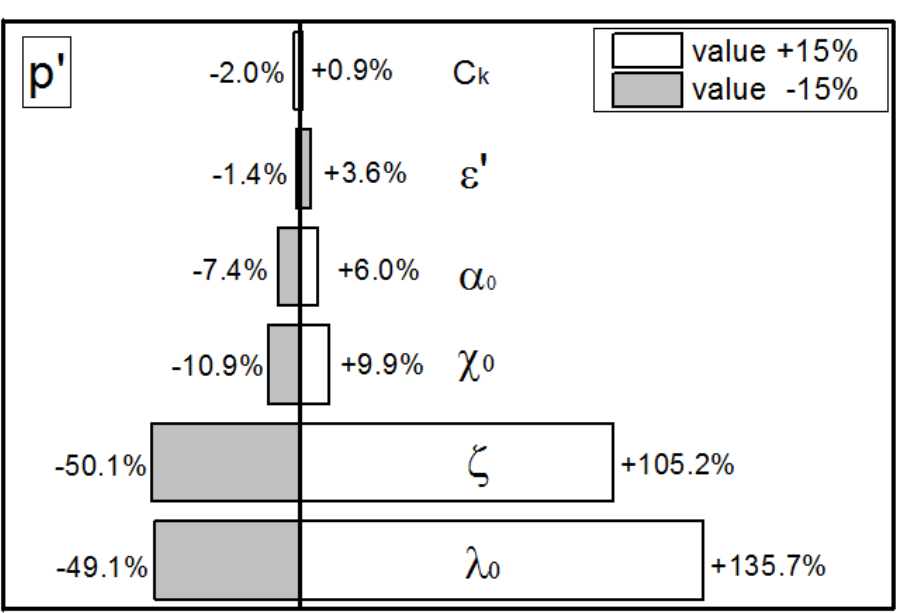

(b)

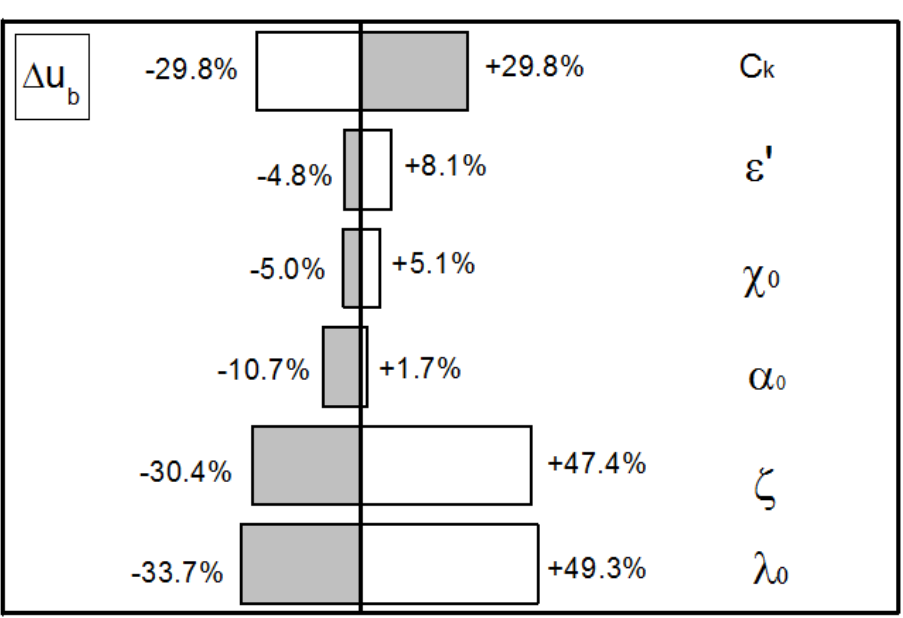

(c)

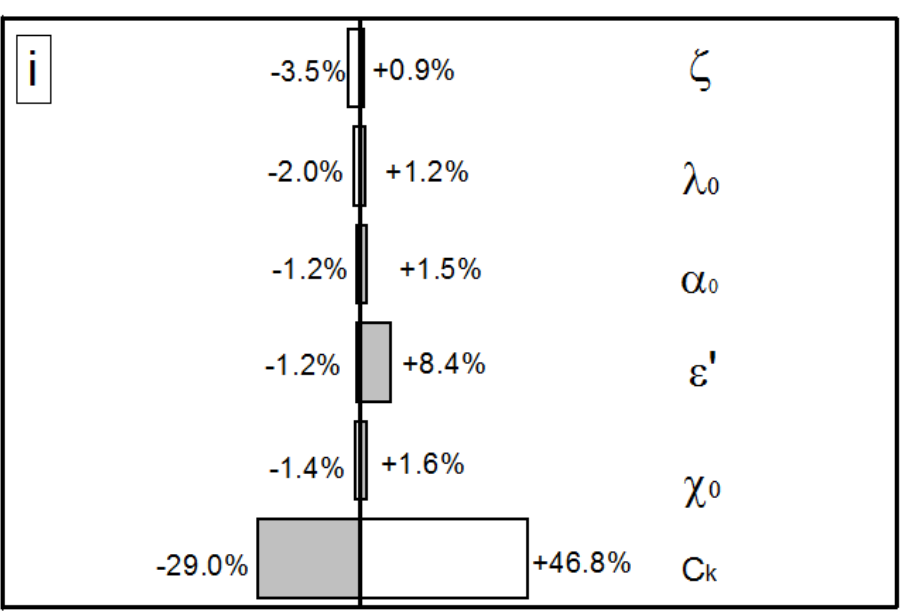

Figure 4.23 Summary of parameter sensitivities for model predictions 


\subsubsection{STRAIN RATE EFFECT ON MODEL PREDICTIONS}

This section investigates the influence of $\dot{\varepsilon}$ of CRS modeling using MEVP-DS. The summary can be seen in Figure 4.24. Figure 4.24 (a) reveals that $\sigma_{c}^{\prime}$ increases with strain rates. Compared to $0.1 \% / \mathrm{hr}, \sigma_{\mathrm{c}}^{\prime}$ is predicted to increase by $21 \%$ and $54 \%$ respectively when strain rates rise to $1 \% / \mathrm{hr}$ and $10 \% / \mathrm{hr}$. Predicted compression curves appear to be parallel to each other, indicating that the predicted compressibility is insensitive to strain rates. Similar conclusions are demonstrated by both laboratory tests and numerical modeling evidence (Leroueil et al. 1985, Yin and Graham 1990).

Figure 4.24 (b) and (c) show that the predicted excess pore pressure response appears to be linearly related to $\dot{\varepsilon}$. Based on the correlation, the allowable strain rate of $1.5 \% / \mathrm{hr}$ is suggested to satisfy the ASTM limit for R (0.03 to 0.15$)$ during the normal consolidation.

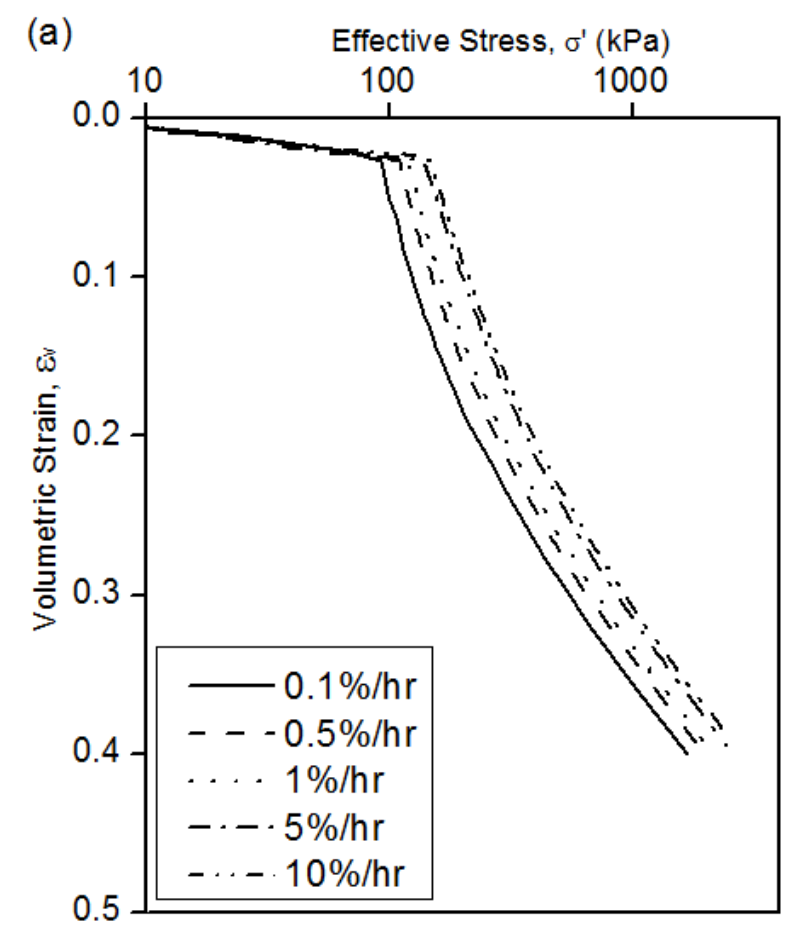



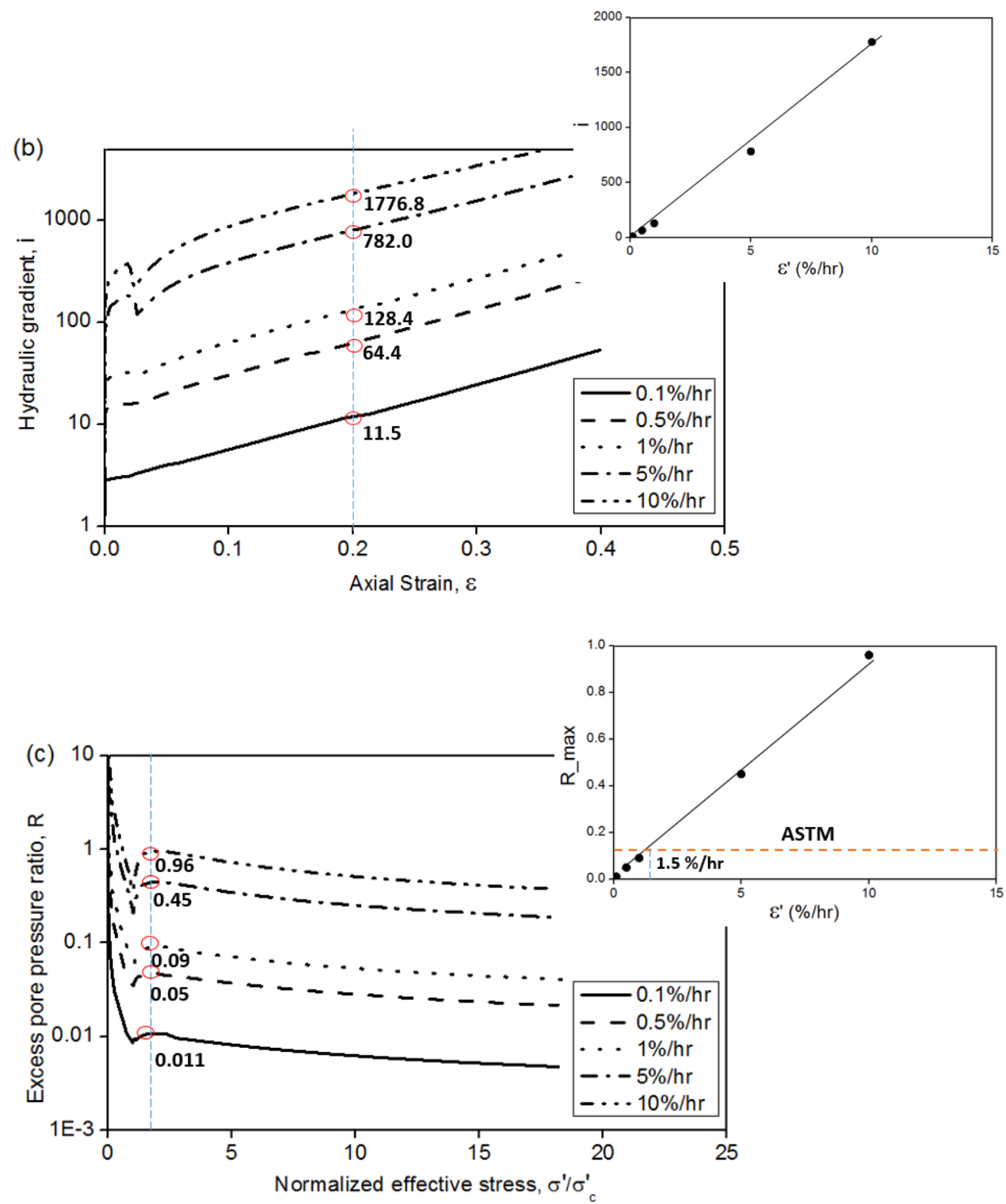

Figure 4.24 Strain rate effects on model predictions

\subsection{SIMULATION OF OEDOMETER TESTS}

This part presents a number of simulations of conventional oedometer tests and 1-D creep tests. The main purpose is to highlight the influence of viscosity on MEVP-DS predictions that has not been demonstrated by CRS modeling. 
Figure 4.25 demonstrates the importance of considering viscosity in modeling conventional oedometer tests on Champlain Sea clay. These oedometer tests were performed on one day intervals.

The result indicates a significant amount of creep that has taken place during oedometer tests. SCLAY1S, which doesn't consider creep, underestimates $\varepsilon_{v}$ by $25 \%$ for the $12.8 \mathrm{~m}$ test and $15 \%$ for the $33.5 \mathrm{~m}$ test. In addition, creep appears to be the most significant factor during the postyielding section. What's more, MEVP-DS provides the prediction almost as well as MEVP, but MEVP-DS performs slightly better for the $33.5 \mathrm{~m}$ test.

Three 1-D creep tests are presented in Figure 4.26. Each test shown here took more than 30 days so that samples can fully manifest its creep behavior. The results demonstrate that MEVP-DS captures the creep behavior better than the rest of models, such as $17.2 \mathrm{~m}$ samples in Figure 4.26 (a) and Figure 4.26 (b). The main improvement can be seen in the primary consolidation section before $1000 \mathrm{~min}$, while other cases all predict a delayed slump of $\varepsilon_{v}$.

It is also noticed that a small EOP (end of primary consolidation) time of Figure 4.26 (c) is predicted by soil models. The reason is that $400 \mathrm{kPa}$ pressure in Figure 4.26 (c) is very close to the $\sigma_{c}^{\prime}$ assigned to this depth, and thus most of the primary consolidation occurs in the elastic range. The models will predict much smaller EOP time and much faster dissipation when it comes to slightly OC cases.

On the other hand, destructuration is still proven to be important as SSC tends to under-predict $\varepsilon_{v}$. S-CLAY1S fails to capture the creep segment after the end of primary consolidation, since the model ignores viscosity. 

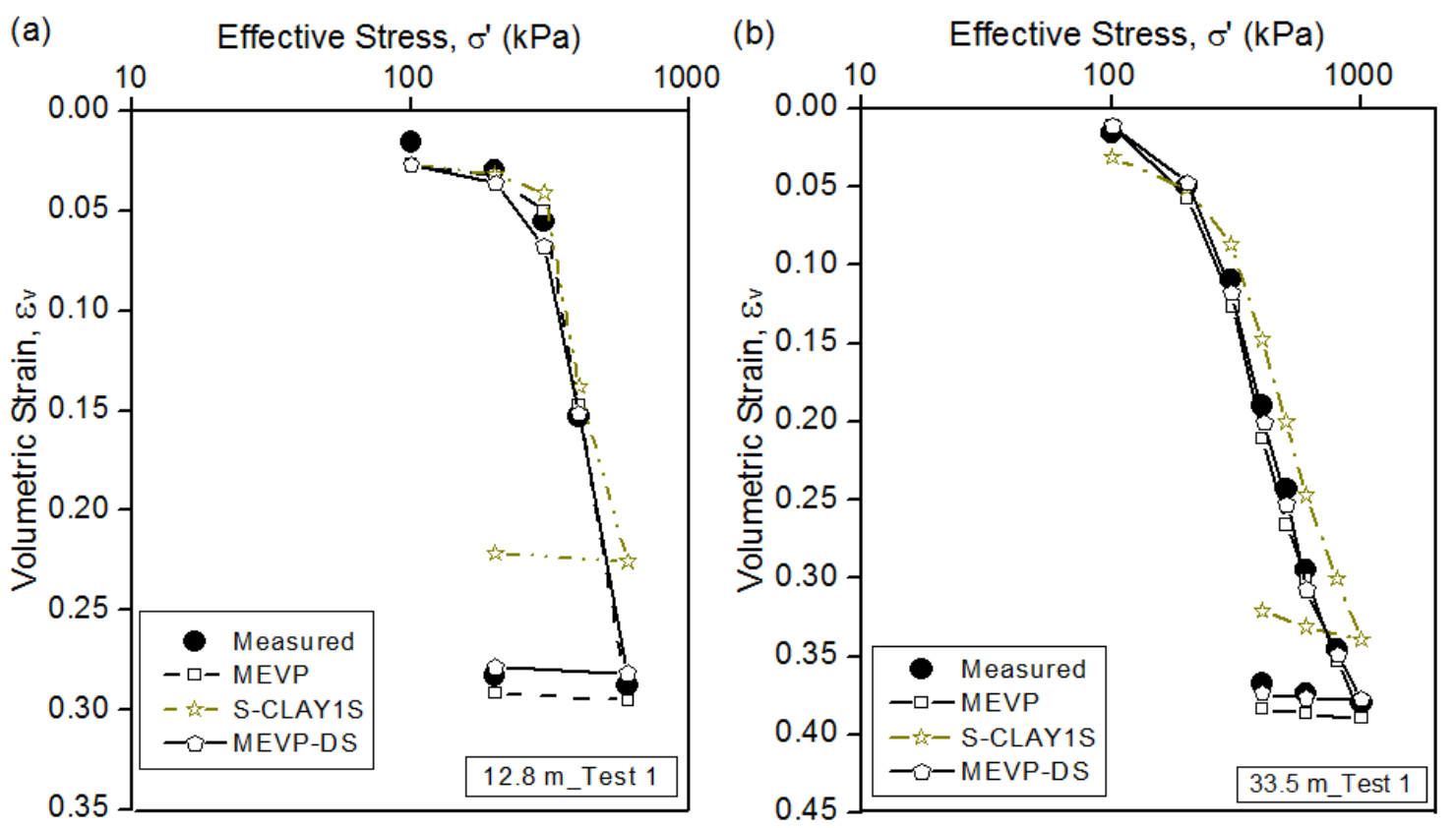

Figure 4.25 Simulation of oedometer tests on the foundation soil 

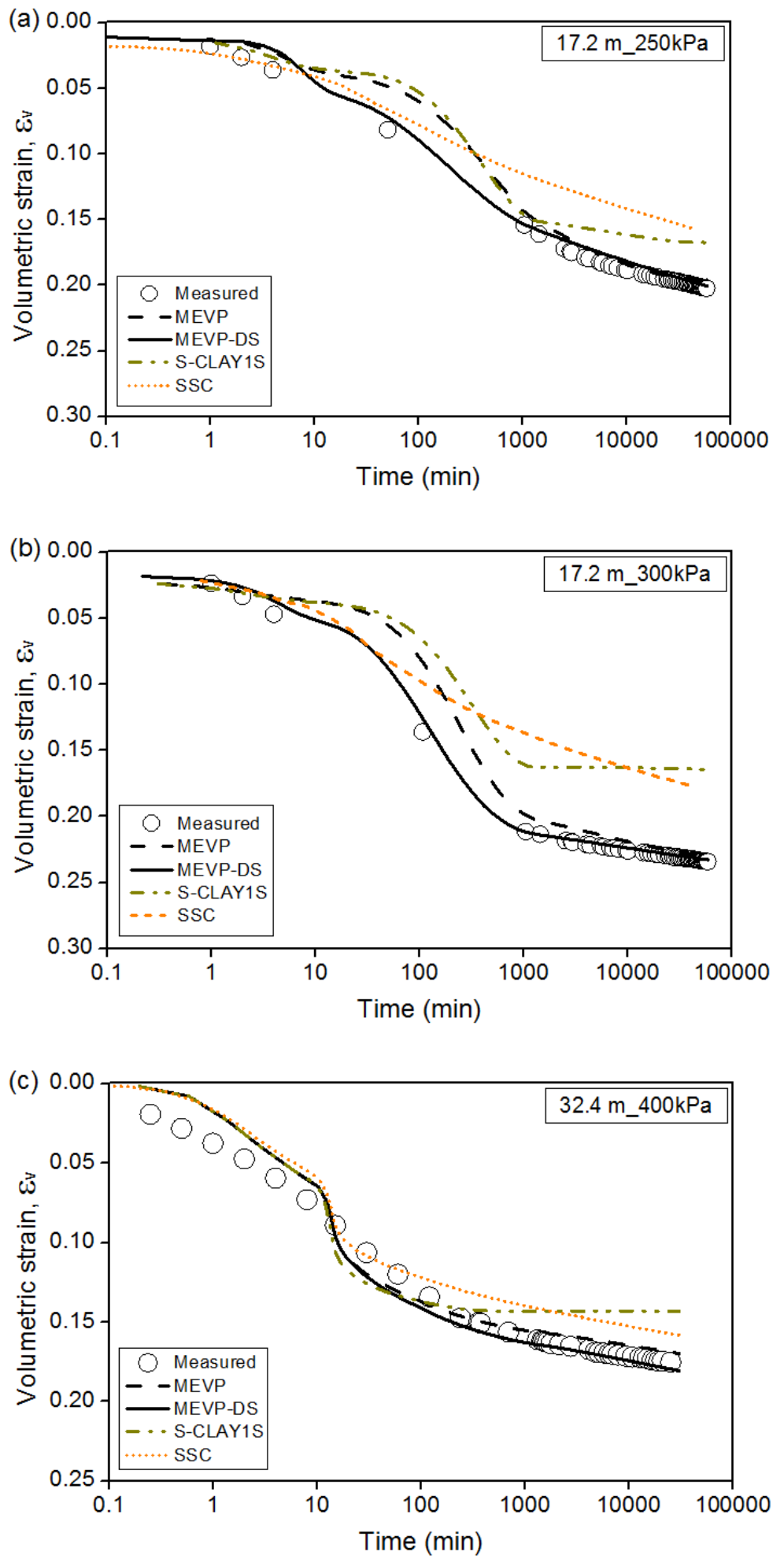

Figure 4.26 Simulation of creep tests on the foundation soil 


\subsection{CONCLUSIONS}

This chapter is to verify the newly proposed MEVP-DS model in the simulation of 1-D consolidation tests of Champlain Sea clay. The simulated tests include CRS, conventional oedometer, and 1-D creep tests. Some conclusions are listed as follows;

1. The predicted $\varepsilon_{v} \sim \log \sigma^{\prime}$ relationship of CRS tests by MEVP-DS is as good as MEVP and S-CLAY1S, while MEVP-DS shows some improvements to excess pore pressure modeling. The same improvement can be also seen in oedometer tests simulation. It is indicated that considering the softening on both intrinsic and structured surface can be beneficial.

2. Model simulations demonstrate that viscosity is not a dominant factor for CRS test modeling. Anisotropy doesn't appear to be influential either, because the yield surface rotation during the test is limited based on the assumed initial yield surface inclination.

3. The parameter study reveals that CRS test predictions are most sensitive to $\varsigma, \lambda_{0}$ and $\mathrm{C}_{\mathrm{k}}$. However, hydraulic gradient $\mathrm{i}$ is more sensitive to $\mathrm{C}_{\mathrm{k}}$ than other compared parameters.

4. Simulations indicate that strain rates lower than $1.5 \% / \mathrm{hr}$ is applicable for Champlain sea clay to satisfy the ASTM standard of $R \leq 0.15$. For other soils with less compressibility, the allowable strain rate can be higher.

However, some more limitations of MEVP-DS model are shown by modeling CRS tests on Champlain Sea clay. When both deviatoric softening and destructuration are considered simultaneously, the model prediction becomes sensitive to $\varsigma$ input. This is because the steepness changes of intrinsic compression lines will affect structured compression curves in a significant way.

Therefore, it is suggested that parameter calibration process may start from $\varsigma$ and $\lambda_{0}$. This is necessary to model intrinsic compression lines at high stress levels well first, before moving on to the calibration of destructuration parameters. 


\section{MODEL VERIFICATION: MODELING SETTLEMENT OF WABA DAM ON SENSITIVE CHAMPLAIN SEA CLAY FOUNDATION}

\subsection{INTRODUCTION}

This chapter is a further verification of MEVP-DS model through a case study by simulating an existing embankment, Waba dam, on sensitive Champlain Sea clay foundation. Embankments built on soft clays are often subject to long-term settlement issues (Bozozuk 1973, Panayides et al. 2012, Fatahi et al. 2013). This is mainly associated with high compressibility (such as destructuration) and viscous behavior (such as creep) of soft soils. Settlement is also accompanied by lateral spreading, which is found responsible for movements and damages of adjacent structures (Stermac et al. 1968, Leussink 1969). However, the accurate prediction of deformation of embankments constructed on soft clay is very challenging (Tavenas et al. 1974, Indraratna et al. 1997).

The idea that creep is resulted from the viscous nature of soil fabric has been widely held by many (Barden 1969, Garlanger 1972, Graham, J. et al. 1983, Yin 2003). To model creep, the timeline concept proposed by Bjerrum (Bjerrum 1967), who laid the groundwork of EVP modeling by separating total settlement into instant and delayed settlement. The timeline approach has been employed by many (Borja and Kavazanjian 1985, Kutter and Sathialingam 1992, Leoni et al. 2008). Yin and Graham (Yin and Graham 1994) extended this approach by introducing the "equivalent time" concept. The model provides fairly encouraging results in predicting the time-dependent behavior of Hong Kong marine clay (Yin and Zhu 1999)

In this chapter, a 2-D plain strain model of a typical cross section of Waba dam is established. The $70 \mathrm{~m}$ thick deposit has been modeled with MEVP-DS. The numerical predictions are compared with field measurements to verify the model. Another purpose is to investigate the contribution of deviatoric softening, creep, destructuration and anisotropy, to the long-term behaviour of Waba dam foundation. This is done by doing parallel simulations using a number of reduced MEPV-DS. 


\subsection{ENGINEERING BACKGROUND}

\subsubsection{LOCATION OF WABA DAM}

Waba dam is located east of the Madawaska River, approximately $65 \mathrm{~km}$ west of Ottawa near the Town of Arnprior, shown in Figure 5.1. It was designed as a containment structure to isolate the reservoir feeding the Arnprior Generating Station (AGS) from entering the Mississippi valley.

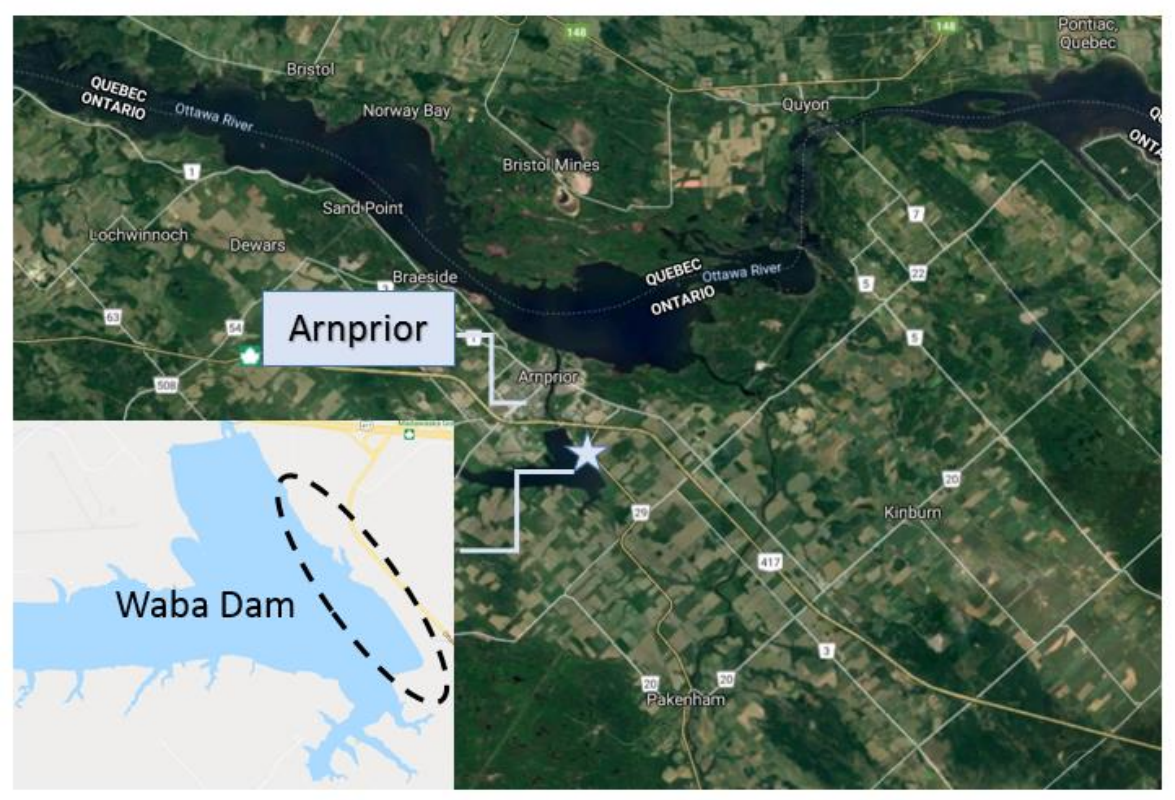

Figure 5.1 Satellite view of the site and its surrounding (Google Map, June $3^{\text {rd }}, 2019$ )

\subsubsection{MAIN FEATURES OF WABA DAM}

The embankment crest is approximately $1,100 \mathrm{~m}$ long and has the maximum height of $18.0 \mathrm{~m}$ above its foundation. It has been divided into three sections according to its cross sections, as shown in Figure 5.2. Area 1 region is situated in the central $190 \mathrm{~m}$ of the dam between Chainages $18+75$ and $24+00$. This area has a crest elevation level of $103.32 \mathrm{~m}$ by design and the maximum height of $18 \mathrm{~m}$ from foundation surface. Area 2 and Area 3 have a less embankment height due to a higher elevation of the foundation surface (Acres Consulting Services 1977). Upstream and downstream weighting berms are $182 \mathrm{~m}$ and $91 \mathrm{~m}$ wide respectively and contain random, mainly impervious soil (Ontario Power Generation 2008). The typical cross section of Area 1 region is 
selected for the FEM modeling discussed in this article since more severe settlements emerged in this area. A $1.5 \mathrm{~m}$ upwards camber was incorporated in the design to accommodate for settlement.

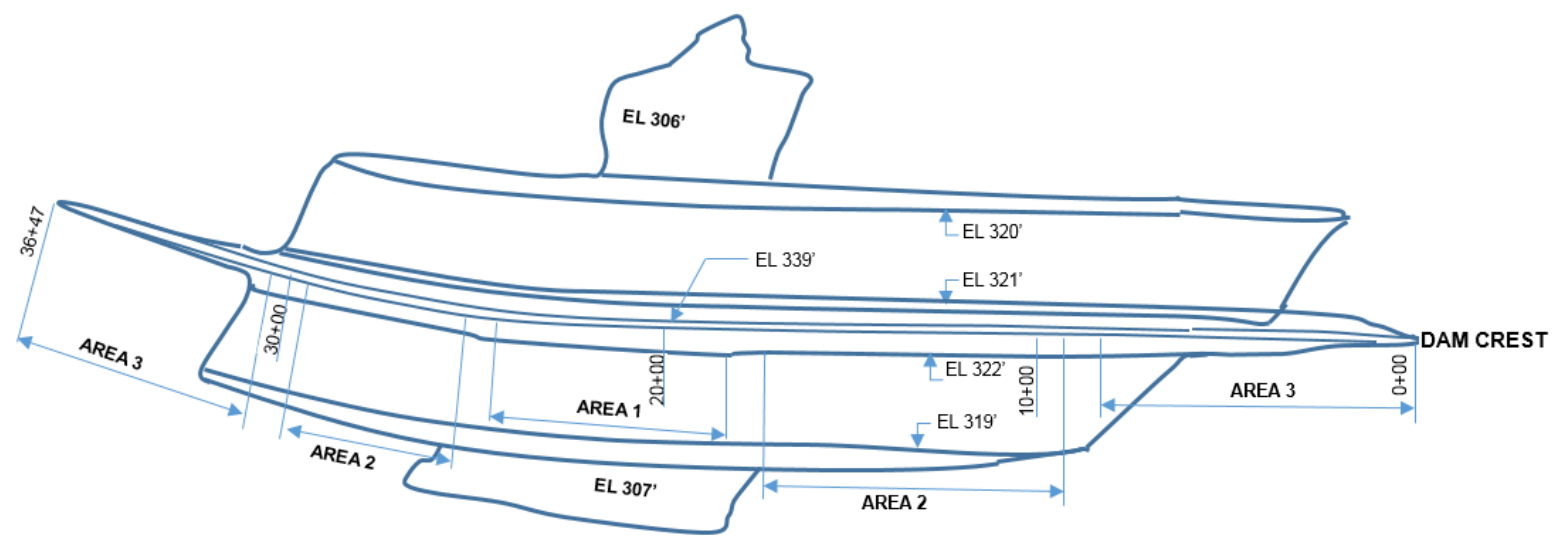

Figure 5.2 Instrumentation of Waba dam (Cragg 1988).

The dam consists of an engineered impervious central clay core comprised of $3 \mathrm{H}$ : $1 \mathrm{~V}$ external slopes with its crest width of $6.0 \mathrm{~m}$, see the typical profile in Figure 5.3. The core was constructed with internal sand filters, drainage zones, an upstream drainage, erosion protection layer to prevent internal erosion of the core zone. A $2.4 \mathrm{~m}$ key trench was excavated along the dam length to extend the core seal through the fissured weathered clay crust.

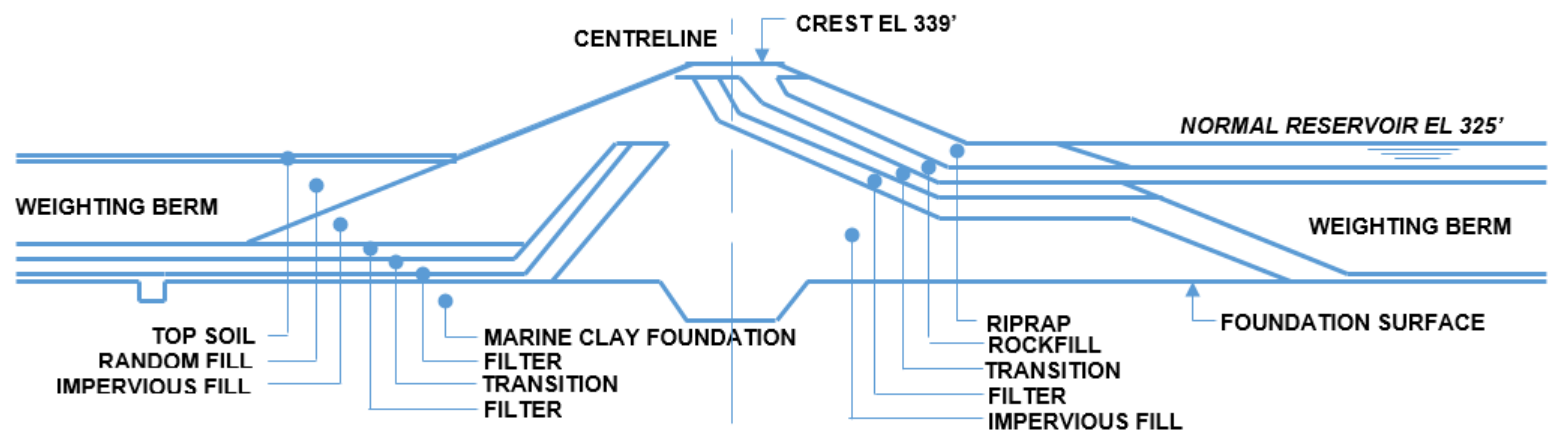

Figure 5.3 Typical cross section of Waba dam (Hydro 1976). 


\subsubsection{CONSTRUCTION SCHEDULE OF WABA DAM}

The dam construction started in September 1974 and suspended in November 1974 for a 6-month winter idle period (Figure 5.4). The construction resumed in May 1975 and completed in November 1975. Due to the detailed records on construction rate are missing, a construction rate of $1.3 \mathrm{~m} / \mathrm{month}$ is assumed in the modeling for the first construction period. A slower rate is assumed in the first construction period to take into consideration the wider coverage of first several layers of weighting berms.

After six months of consolidation for the winter idle period, a construction rate of $2.3 \mathrm{~m} / \mathrm{month}$ is resumed subsequently until the $18 \mathrm{~m}$ crest is accomplished. This phase is assumed to proceed much faster than the first six months.

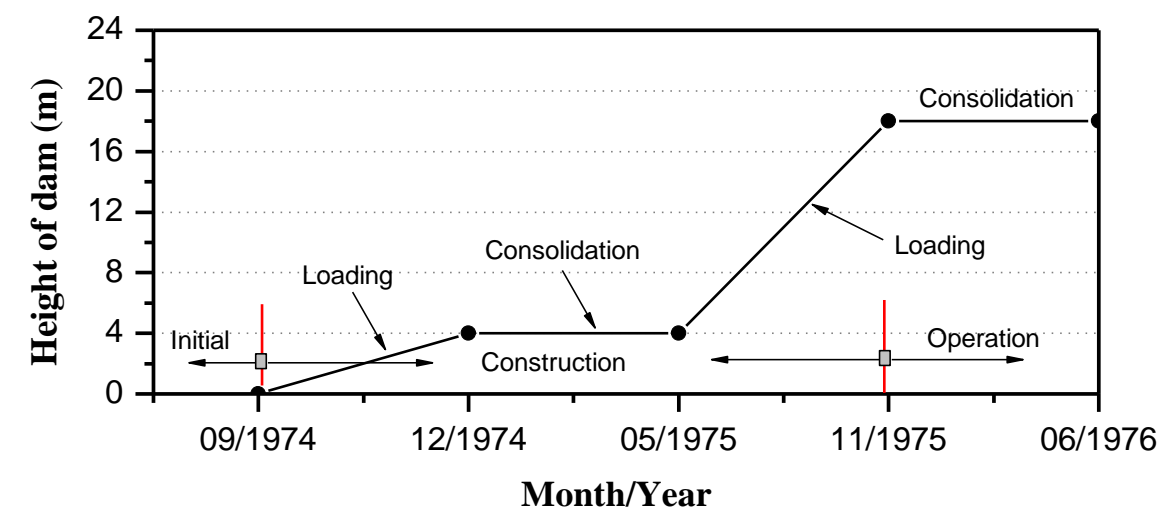

Figure 5.4 Dam construction sequences used in the numerical analysis

\subsubsection{INSTRUMENTATIONS AND MEASUREMENTS}

Instrumentation and measurements were employed since the construction commencement to monitor settlements, lateral spreading and pore pressure dissipation. Instrumentations involve 7 slope indicators ( 6 in berm and 1 in the center portion of dam), 11 foundation surface settlement 
gauges (for monitoring foundation settlement), 1 borehole settlement gauge, 28 piezometers, and 25 surface monuments (for crest settlements).

As shown in Figure 5.5, these instrumentations were installed throughout the longitudinal direction of the dam in 1975. Most of the instrumentation was located in Area 1 region. Surface monuments intended for measuring dam crest settlement have been performing in a satisfactory manner in the early stage. 6 of the 25 surface monuments were deployed in Area 1 and were used for comparison with model predictions later on. However, some slope indicators monitoring upstream movement were gradually abandoned due to the deterioration of casings. Piezometers were also found to degrade over time. In 2015, six new piezometers were installed in two boreholes where the intact soil samples were retrieved for lab testing. Some of the test results have been discussed in Chapter 4. More details will be presented in section 5.3.
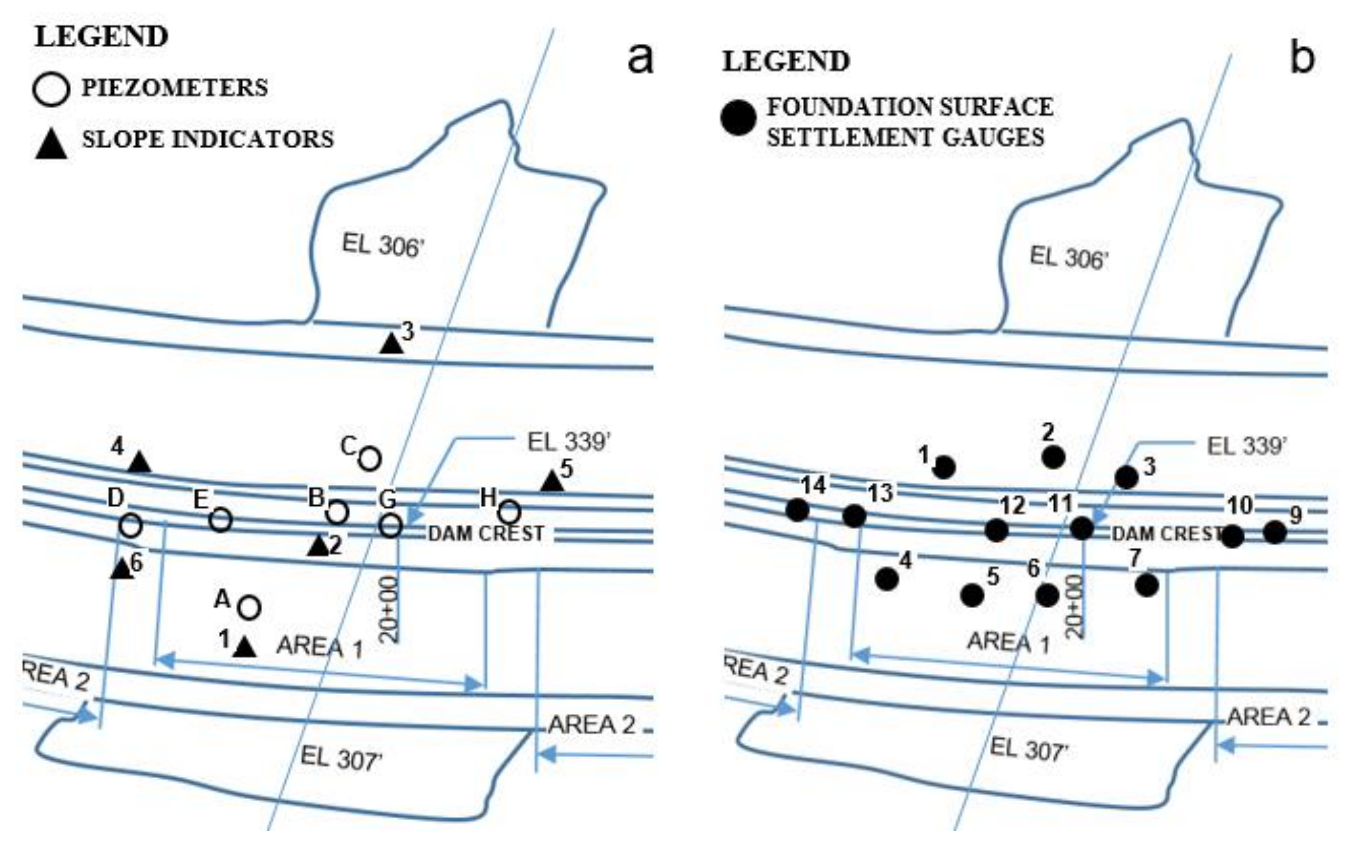

Figure 5.5 Instrumentation plan in Area 1 region 


\subsection{GEOTECHNICAL CHARACTERISTICS OF CHAMPLAIN SEA CLAY}

\subsubsection{PHYSICAL PROPERTIES OF THE SOIL SAMPLES}

Champlain Sea clay samples were retrieved in high quality by the Laval sampler in 2015. Clay cores were cut into sub-samples with a nominal length of $580 \mathrm{~mm}$ and a diameter of $220 \mathrm{~mm}$. Figure 5.6 presents typical soil samples located in the upper clay and the lower clay area. The upper clay is defined as the top $32 \mathrm{~m}$ below the foundation surface, followed by the lower clay located at a deeper region from $32 \mathrm{~m}$ to $69.5 \mathrm{~m}$. The color of clay was mainly grey, varying from dark gray, greenish grey, and grey.

Most clay samples were observed to be homogeneous. Appearance of thin beds of sand were found in a lower clay samples. In a few samples, fish shells were observed which confirmed the marine clay origin of Champlain Sea clay. The consistency of the upper clay is firm and relative easy to trim for sample preparations. The consistency of lower clay is stiff and much difficult for sample preparations due to its brittleness (Liu et al. 2017)

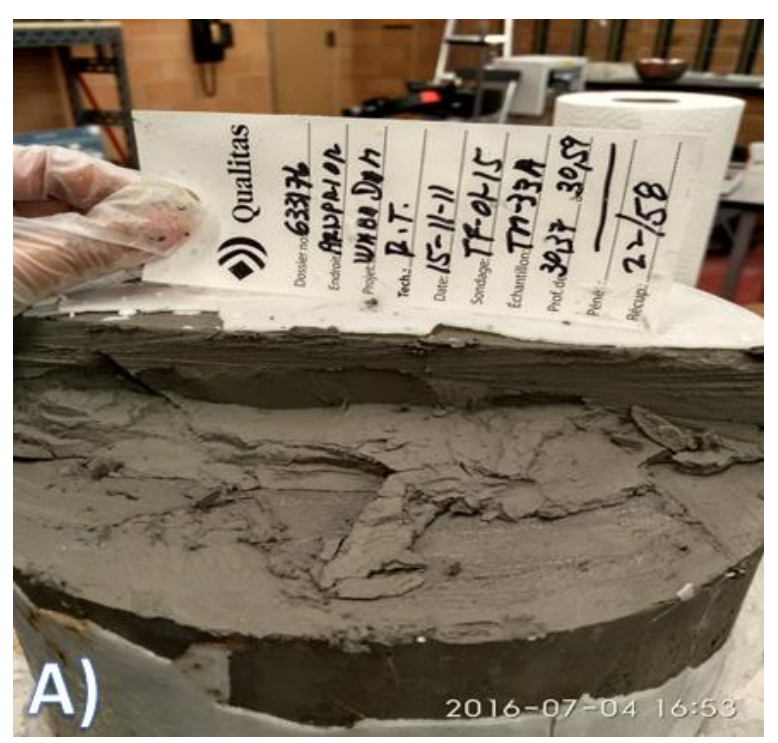




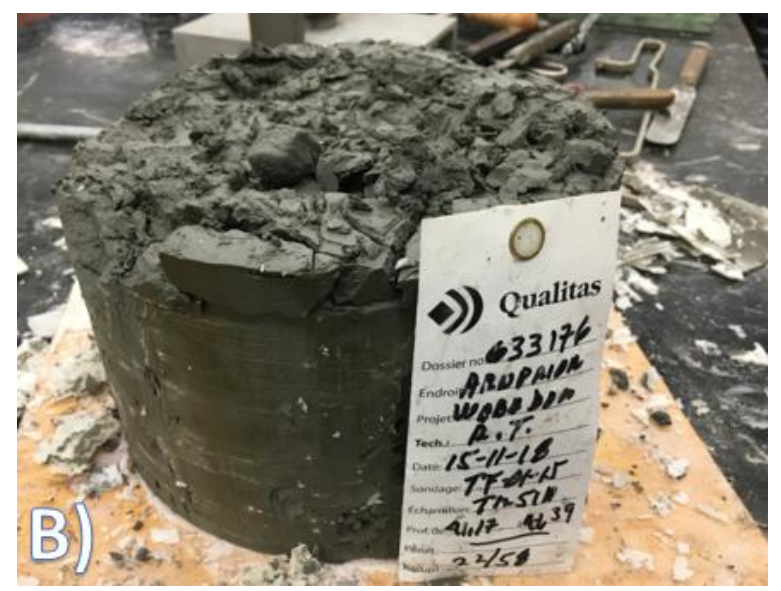

Figure 5.6 Examples of Champlain Sea clay taken from Waba dam foundation

\subsubsection{MOISTURE CONTENTS AND NATURAL DENSITIES}

Moisture contents of the clay foundation were collected in accordance with ASTM D2216. The results from 2017 investigation were plotted together with 1987 Investigation in Figure 5.7. It is tangible that the water content decreases from a typical value of $80 \%$ close to the ground surface to roughly $40 \%$ in lower clay area. Void ratios were calculated based on a typical specified gravity of 2.75 observed throughout the foundation depths

The Atterberg Limits were also determined for soil samples from different depths. The Liquid Limits (LL) were yielded according to ASTM D 423, and Plastic Limit (PL) according to ASTM D 424. The LL values are found to be typically lower than the water contents collected at according depths.

Additionally, a USCS plasticity chart was produced using the results of Atterberg Limit tests, as shown in Figure 5.8. Most of the tested samples show a clear cluster of $\mathrm{CH}$, with only a few points moving slightly into CL.

Soil density were derived by direct weight and volume measurements. Average values of multiple measurements for each depth are shown in Figure 5.9. The density results reveal a clear stratification of the foundation. The upper clays appear to possess a lower bulk density of approximately $1600 \mathrm{~kg} / \mathrm{m}^{3}$. This is in contrast to the values increasing from 1600 to $1900 \mathrm{~kg} / \mathrm{m}^{3}$ over depths observed in the lower clay region. 

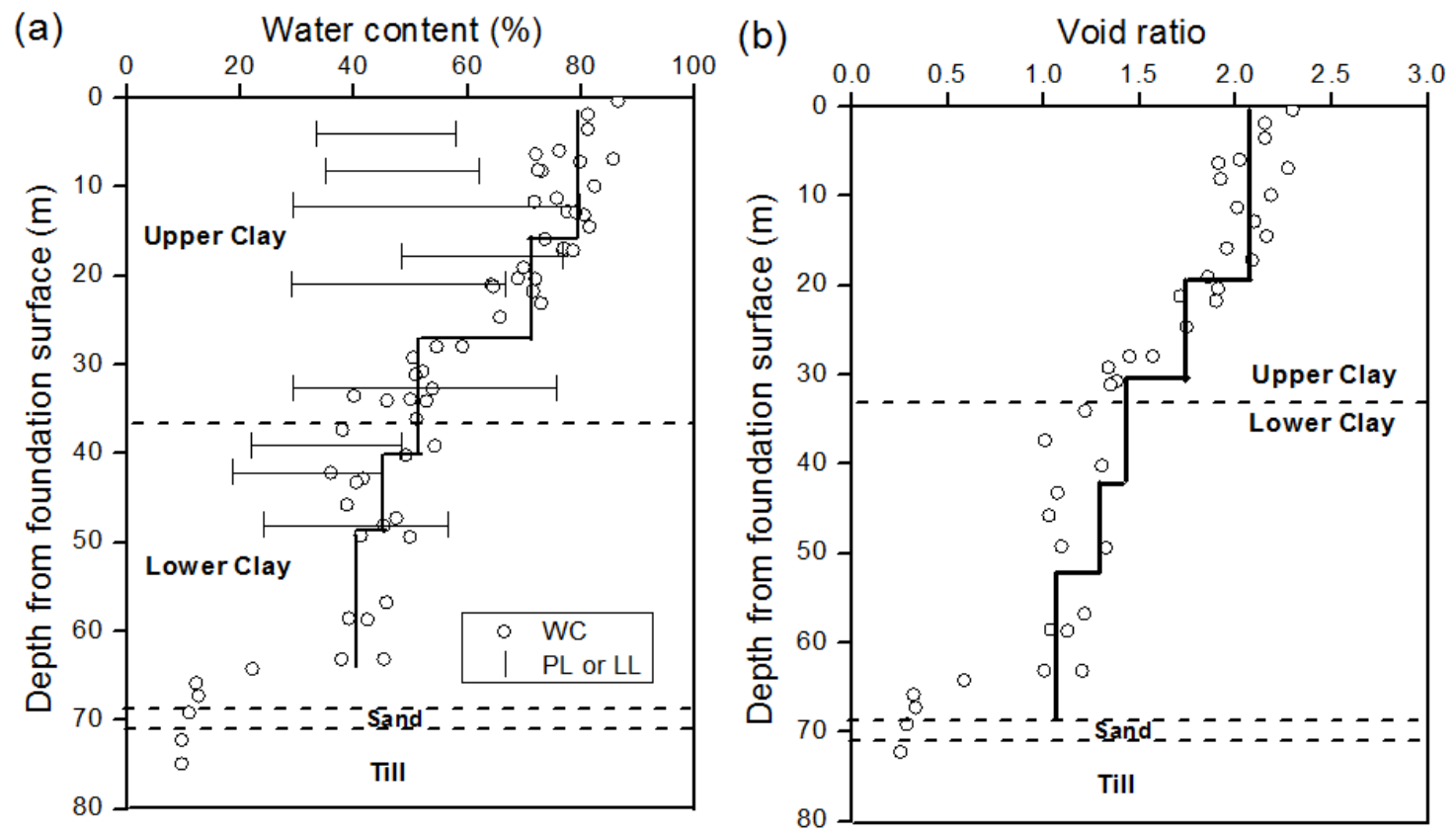

Figure 5.7 Water content, Atterberg limits and void ratio profiles of the clay foundation

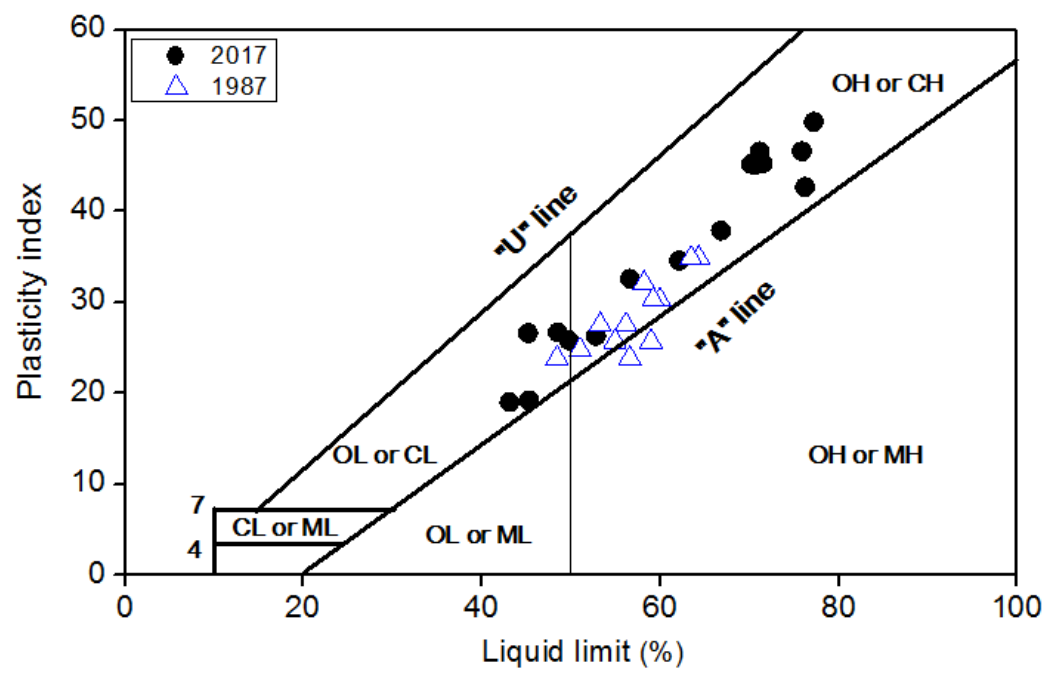

Figure 5.8 Plasticity chart of foundation clays of Waba dam 


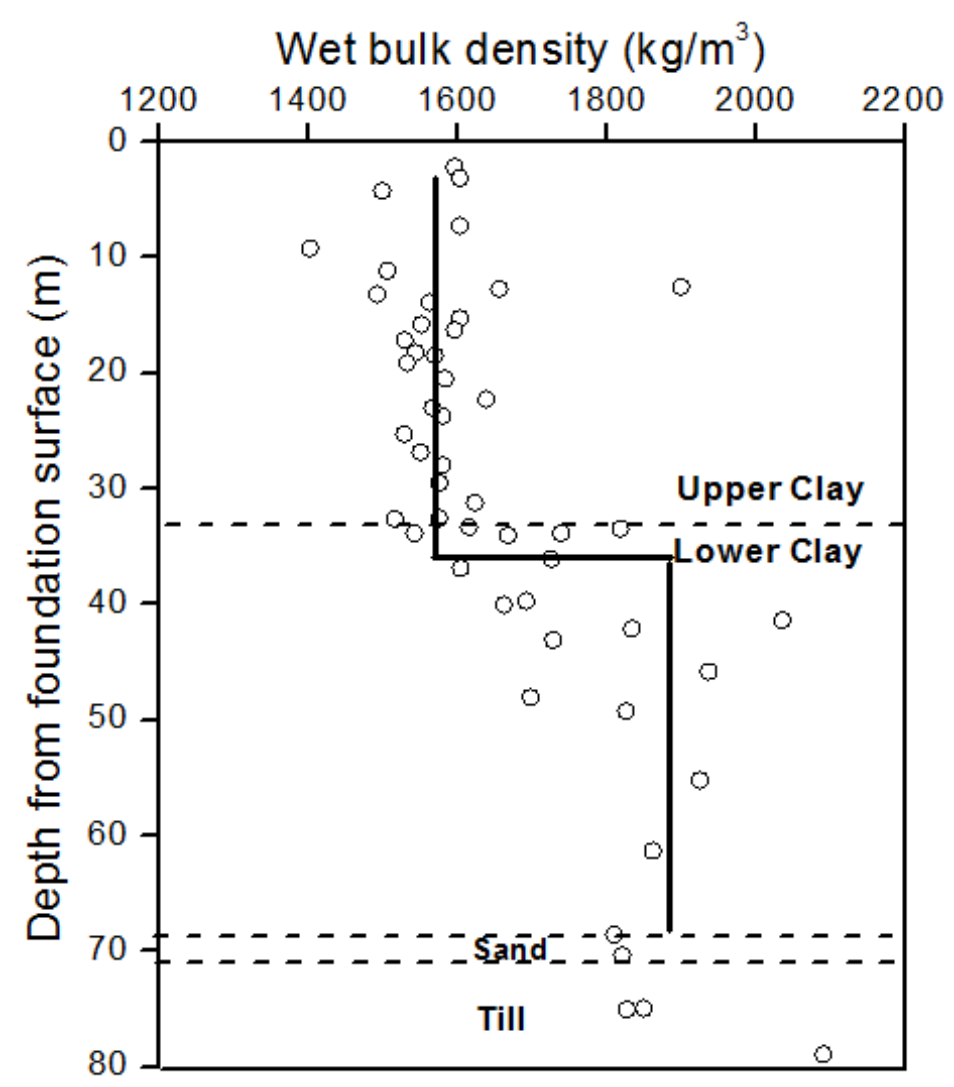

Figure 5.9 Bulk density of Waba dam foundation

\subsubsection{UNDRAINED SHEAR STRENGTH AND SENSITIVITY}

Undrained shear strength was determined by means of both MV (minivane shear) tests, UCS (unconfined compression) tests, and UU (unconsolidated-undrained triaxial) tests. The results are plotted in Figure 5.10. In the figure, $U U-H C$ denote the samples cut horizontally, and $U U-45^{\circ}$ denote the samples cut intentionally in $45^{\circ}$. This practice was to investigate the potential shear strength anisotropy. It is unclear which tests $Q U$ stands for, but it could be a type of vane shear tests performed by 1987 investigation.

Sensitivity, $S_{t}$, was determined by dividing the undisturbed shear strength by the remoulded shear strength. The undrained shear strengths of undisturbed and fully remoulded samples were conducted by miniature vane shear tests according to ASTM D4648. The $S_{t}$ profile of the foundation is shown in Figure 5.10 (b), with the obtained $S_{t}$ ranging between 7.2 and 14.9. 
Based on Canadian Foundation Engineering Manual (CGS 2006), the foundation clay can be classified as medium sensitivity $\left(10<\mathrm{S}_{\mathrm{t}}<40\right)$. However, based on a more detailed Canadian classification (Holtz et al. 2010), most of the depths tested have the sensitivity falling into the extremely sensitive category.
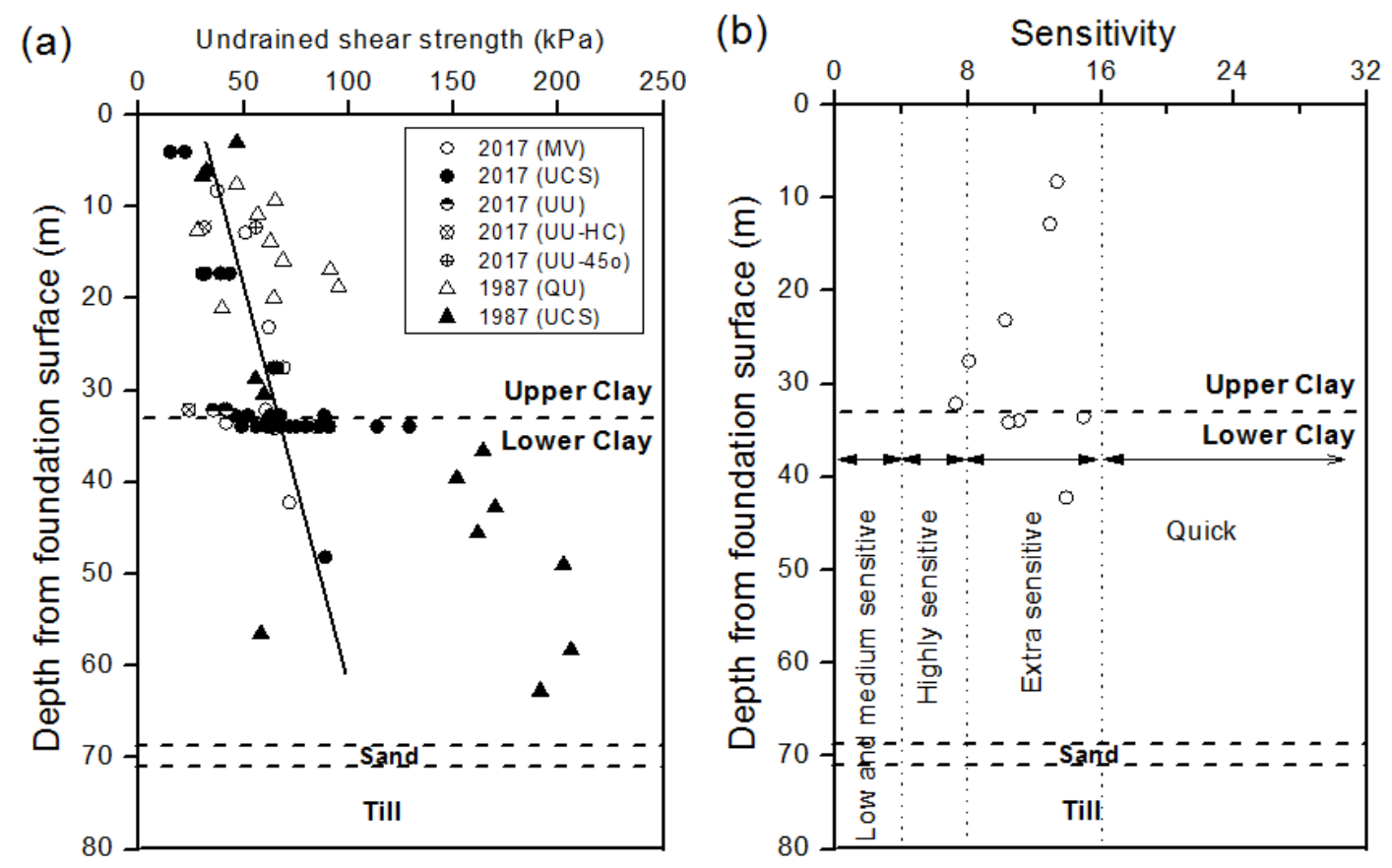

Figure 5.10 Undrained shear strength profile of Waba dam foundation

\subsubsection{CRITICAL STATE SOIL PROPERTIES}

A total of 25 undrained triaxial compression tests, aiming to determine $M$, have been performed on foundation clays. Figure 5.11 shows the summary of critical state points of both normally consolidated (NC) and over consolidated (OC) samples. For over consolidated triaxial tests, soil samples were consolidated at confining pressures lower than their estimated mean yield stresses. These mean yield stresses were calculated from pre-consolidation pressures identified by CRS tests. The critical state point for each test was determined by the maximum stress ratio $\sigma_{1} / \sigma_{3}$ criterion.

The test results indicate that NC upper clay exhibits a slightly higher critical state friction $\left(\varphi^{\prime}\right)$ angle of $33^{\circ}(M=1.33)$ than the lower clay of $30^{\circ}(M=1.30)$. Additionally, an insignificant 
amount of cohesion $\left(c^{\prime}\right)$ has been observed in NC samples. Note that some underestimation in $M$ could be possible if $c^{\prime}$ is considered in the interpretation of test results.
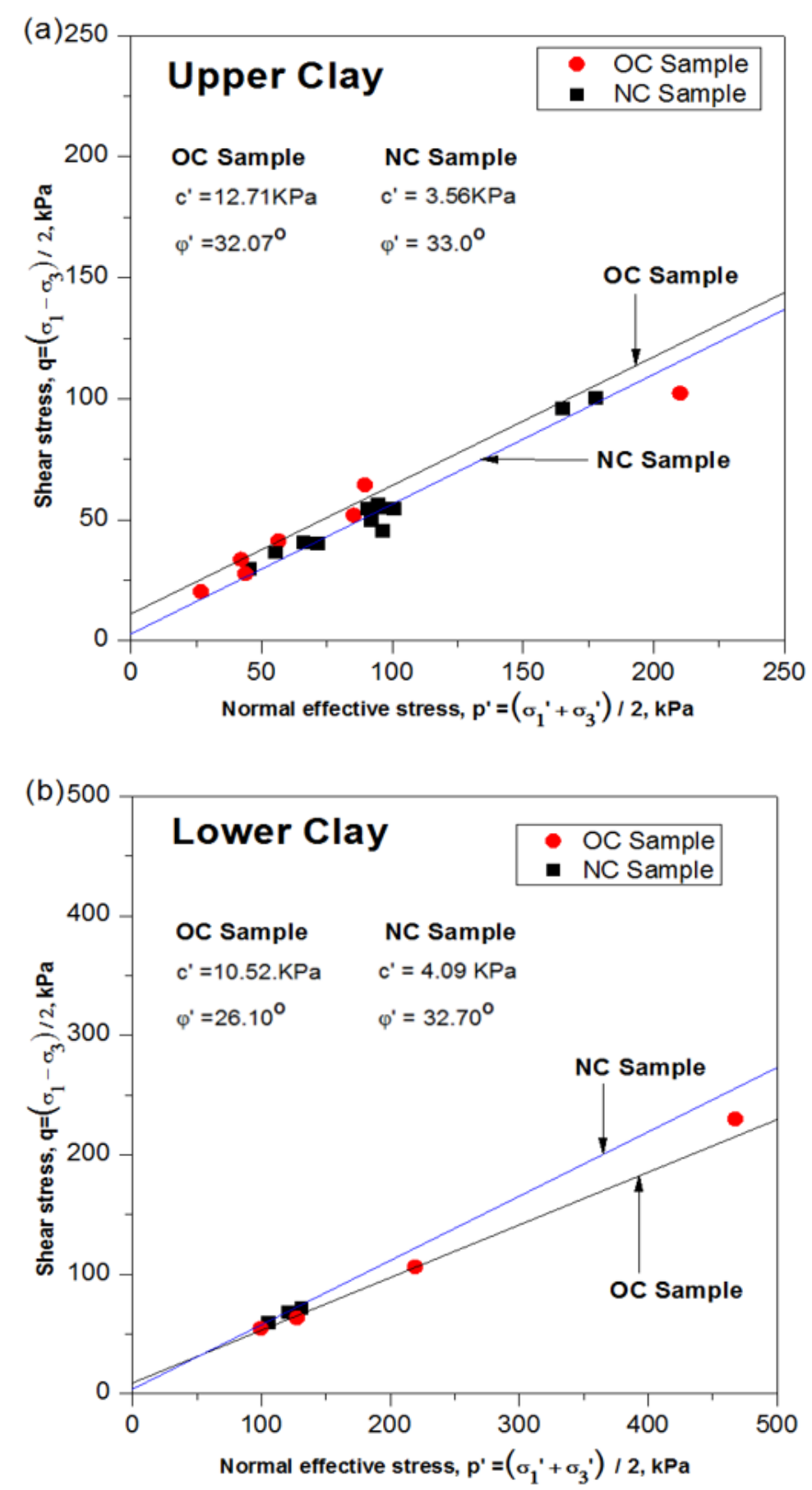

Figure 5.11 Summary of critical state points of Waba dam foundation (Liu et al. 2017) 


\subsubsection{PRECONSOLIDATION PRESSURE AND OCR}

Figure 5.12 (a) presents the profile of $\sigma_{c}^{\prime}$ determined from conventional oedometer tests conducted in 1972. The results of oedometer tests and CRS consolidation tests conducted in 2017 investigation are also presented. Some growth in $\sigma_{c}^{\prime}$ has been observed at $12 \mathrm{~m}$ depth and the deeper area when comparing 1972 and 2017 results.

In addition, a high value of $400 \mathrm{kPa}$ has been reported by 1972 close to the ground surface. It demonstrates a stiff surface layer potentially owing to aging, weathering, and unloading in the geological history. This leads to an OCR of 20 shown in Figure 5.12 (b). Overall, the presence of over-consolidation is observed for upper clay, with OCR ranging from 1.5 to 4.4. However, OCR values are found to decrease with depth, until stabilizing at 1 at $25 \mathrm{~m}$ or deeper depth.
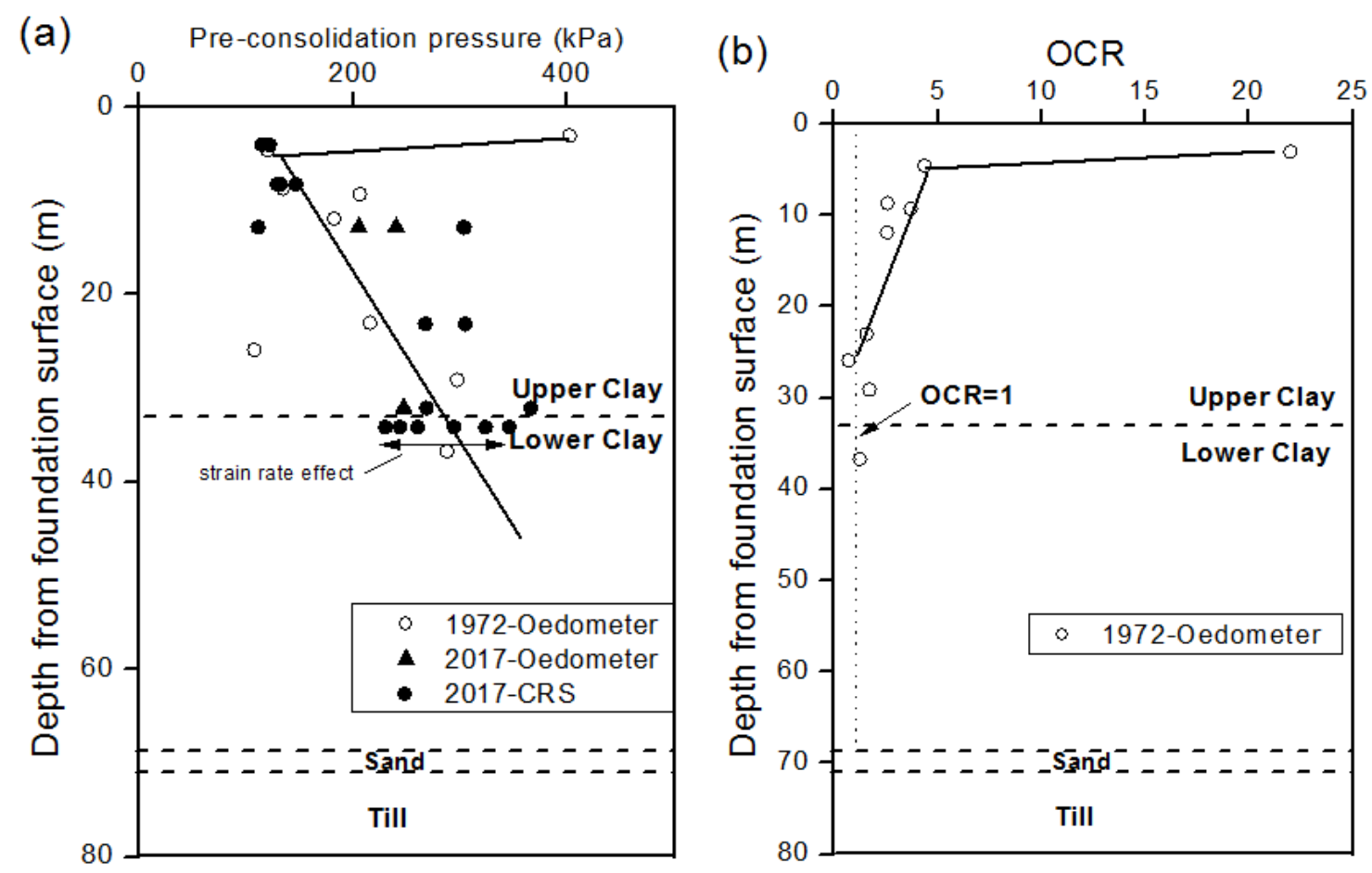

Figure 5.12 Pre-consolidation pressure profile of Waba dam foundation

\subsubsection{SALINITY}

The salinity level has been observed throughout the deposit, as shown in Figure 5.13. The salinity in dry soil $(\mathrm{g} / \mathrm{kg})$ and the salinity in the pore fluid $(\mathrm{g} / \mathrm{L})$ are two expressions used to present the 
data. The test results report a low salinity level of less than $5 \mathrm{~g} / \mathrm{L}$ close to the foundation surface. The values ascend with depth, reaching around $20 \mathrm{~g} / \mathrm{L}$ in the lower clay. Moreover, it is also observed that the salinity from the diluted fluid method is about 4-5\% higher than the one from the squeezed fluid method.
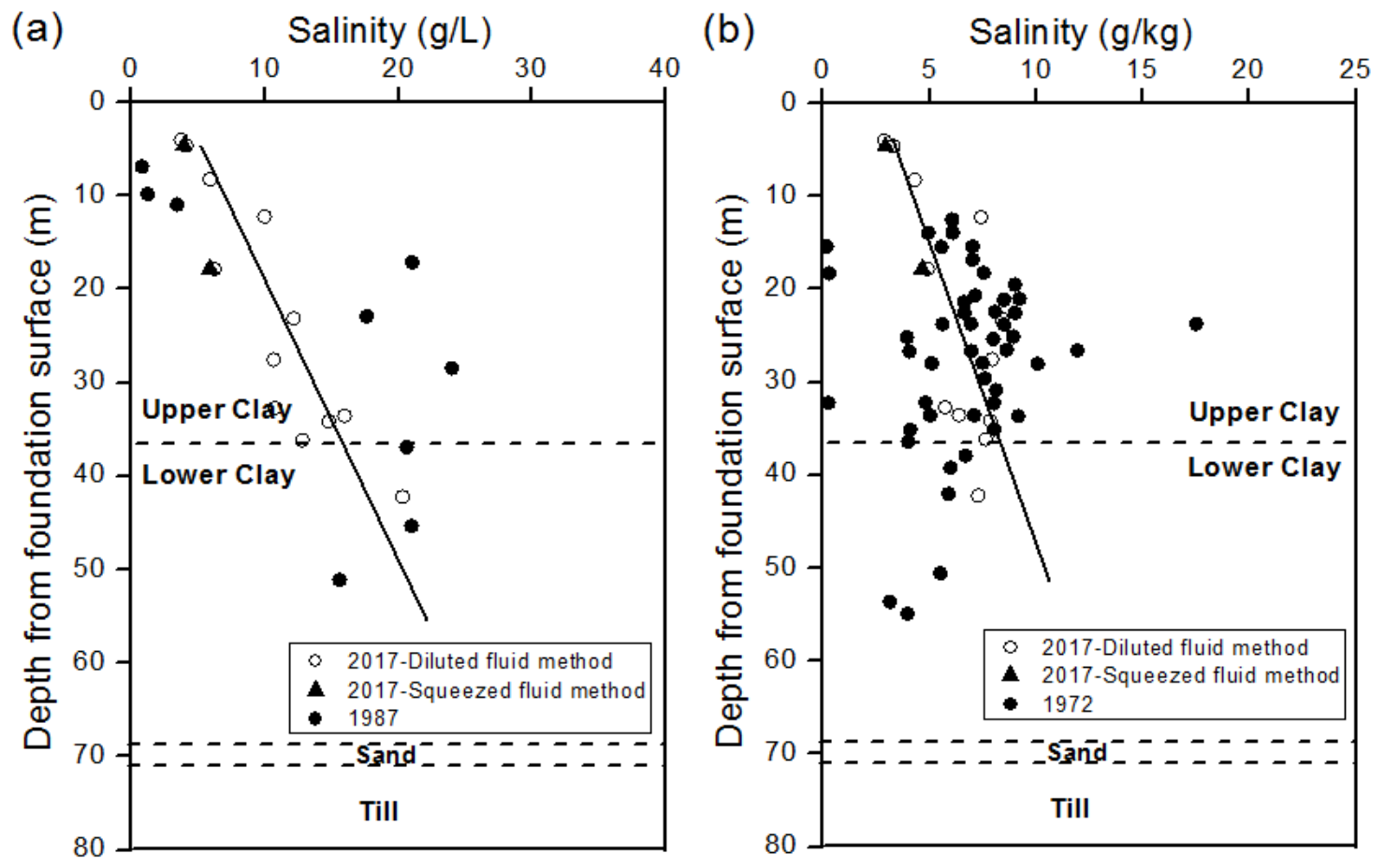

Figure 5.13 Salinity profiles of Waba dam foundation

\subsection{GEOMETRY AND MODEL DETAILS OF WABA DAM}

The FEM analysis focuses on the Area 1 region of Waba dam with the deepest Waba depression. A typical cross section has been selected to represent the geometry of this region. Figure 5.14 has more details. The foundation surface was at EL: $85.3 \mathrm{~m}$. The $18 \mathrm{~m}$ high dam was built accompanied by $12 \mathrm{~m}$ thick weighting berm underlain by a $69.5 \mathrm{~m}$ deep marine clay foundation. The marine layers are underlain by a $1 \mathrm{~m}$ thick sand layer and $10 \mathrm{~m}$ thick till layer.

The horizontal boundary is $500 \mathrm{~m}$ away from the dam centreline to marginalize boundary effects

of the model. The geometry is slightly asymmetrical as the width of the left berm is greater than 
the right. Both horizontal and vertical displacements are fixed at the bottom boundary, and horizontal displacement are fixed at both lateral boundaries. The foundation is allowed to drain at lateral boundaries but the drainage is closed at the bottom boundary to allow for the bedrock. The foundation surface is also opened for drainage. Even so, it is noticed from the simulation that excess pore pressure in bottom layers drain horiztonally in a rather fast way, which is due to the presence of the highly permeable sand layer.
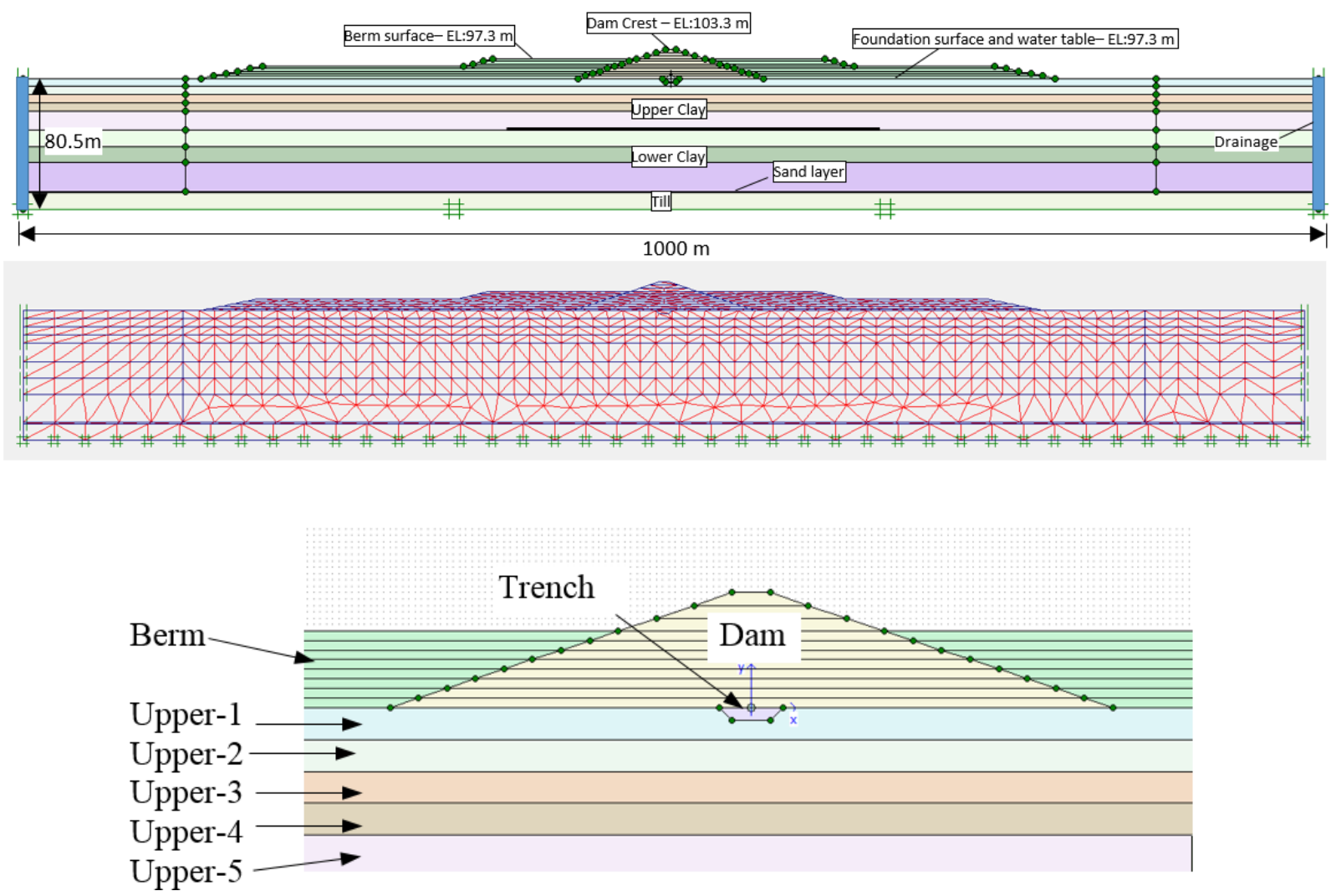

Figure 5.14 FEM geometry of Area 1 cross section

The $69.5 \mathrm{~m}$ thick deposit has been partitioned into 8 sublayers. The upper five layers compose the upper clay ranging from foundation surface to $32 \mathrm{~m}$ depth. The lower clay is composed of three layers from $32 \mathrm{~m}$ to the sand layer. A trench ( $2 \mathrm{~m}$ in depth) is excavated at the foundation surface along the centreline of the dam following the construction records. Around 1900 triangular plane strain elements are employed by PLAXIS 2D implemented with MEVP-DS and other four soil 
models as user-defined models. Mesh-sensitivity analysis has been performed to ensure the accuracy of model estimations.

\subsection{MODEL PARAMETERS FOR FOUNDATION CLAY}

Similar to Chapter 4, five soil models are employed in the embankment analysis, including MEVPDS, MEVP, EVP+S-CLAY1, EVP, and S-CLAY1S. Table 5.1 specifies the detail of these models. The linear-perfectly plastic Mohr-Coulomb model has been used for modeling weighting berm, dam, sand layer, and till, see the summary of parameters in Table 5.2. This assumption has been made in that the embankment was made from the well-compacted relocated clay from the nearby project. Due to the excavation and re-compaction, the sensitive soil structure would be nearly removed. Creep has not been considered in the embankment material either. Model parameters for the clay foundation are summarized in Table 5.3 and

Table 5.4.

Foundation soil parameters are derived from laboratory test and calibrated by test simulations presented in Chapter 4. $\sigma_{c}^{\prime}$ input are from oedometer tests from 1972 Investigation and have been adjusted by temperature effects proposed by Leroueil (Leroueil 1996). The coefficient of earth pressure at rest $K_{0}$ was estimated using the correlation $K_{0}=(1-\sin \phi) O C R^{0.95}$ proposed in (Lefebvre et al. 1991, Hamouche et al. 1995).

Table 5.1 Details of all six cases of parameter inputs for the foundation clay

\begin{tabular}{lll}
\hline Case Name & Details & Comments \\
\hline MEVP-DS & $\begin{array}{l}\text { Considers deviatoric softening, viscosity, destructuration } \\
\text { and anisotropy }\end{array}$ & The modified MEVP \\
MEVP & Considers viscosity, destructuration and anisotropy & MEVP \\
EVP+S-CLAY1 & Considers viscosity and anisotropy & Reduced version of MEVP \\
EVP & Considers only viscosity & Reduced version of MEVP \\
S-CLAY1S & Considers destructuration and anisotropy & Reduced version of MEVP \\
\hline
\end{tabular}


Table 5.2 M-C model parameters used for weighting berm, dam, sand and till layer

\begin{tabular}{ccccccccc}
\hline Layer & $\begin{array}{c}\text { Depth } \\
\mathrm{m}\end{array}$ & $\begin{array}{c}\boldsymbol{E}_{\boldsymbol{s}} \\
\mathrm{MPa}\end{array}$ & $\begin{array}{c}\boldsymbol{\gamma} \mathrm{kN} / \mathrm{m}^{3} \\
\mathrm{kPa}\end{array}$ & $\begin{array}{c}\boldsymbol{c} \\
\circ\end{array}$ & $\begin{array}{c}\boldsymbol{\psi} \\
\circ\end{array}$ & $\begin{array}{c}\boldsymbol{v} \\
-\end{array}$ & $\begin{array}{c}\mathbf{k} \\
\times 10^{-7} \mathrm{~m} / \mathrm{s}\end{array}$ \\
\hline Berm & - & 30 & 15 & 20 & 20 & 0 & 0.35 & 1 \\
Dam & - & 30 & 19 & 20 & 20 & 2 & 0.35 & 1 \\
Sand & 69.5 to 70.5 & 100 & 20 & 1 & 35 & 0 & 0.3 & 150 \\
Till & 70.5 to 80.5 & 100 & 21 & 5 & 38 & 0 & 0.3 & 15 \\
\hline
\end{tabular}

Table 5.3 Model parameters used for MEVP, EVP+S-CLAY1, EVP, and S-CLAY1S

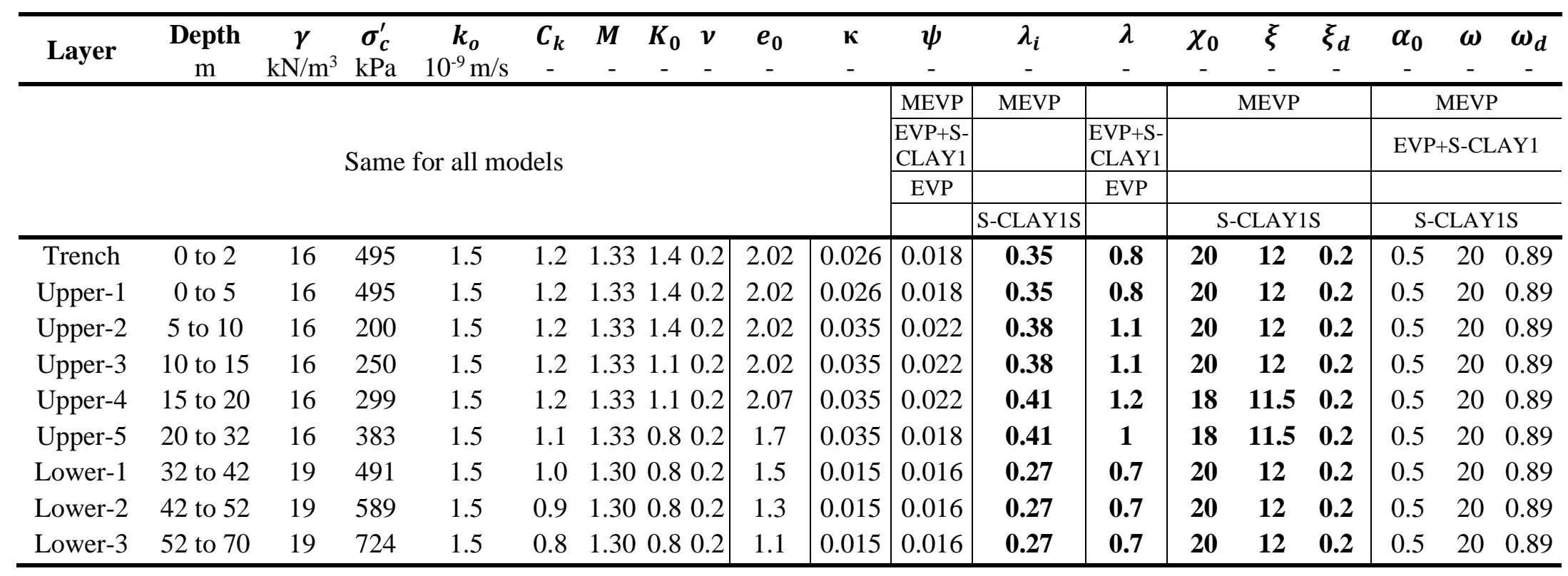


Table 5.4 MEVP-DS model parameters used for clay foundation and trench backfill

\begin{tabular}{|c|c|c|c|c|c|c|c|c|c|c|c|c|c|c|c|c|c|c|c|}
\hline Layer & $\begin{array}{c}\text { Depth } \\
\text { m }\end{array}$ & $\begin{array}{c}\boldsymbol{\gamma} \\
\mathrm{kN} / \mathrm{m}^{3} \\
\end{array}$ & $\begin{array}{r}\boldsymbol{\sigma}_{\boldsymbol{c}}^{\prime} \\
\mathrm{kPa} \\
\end{array}$ & $\begin{array}{c}\boldsymbol{k}_{\boldsymbol{o}} \\
\times 10^{-9} \mathrm{~m} / \mathrm{s} \\
\end{array}$ & $\begin{array}{c}C_{k} \\
- \\
\end{array}$ & $\begin{array}{r}M \\
- \\
\end{array}$ & $\begin{array}{c}K_{0} \\
- \\
\end{array}$ & $\begin{array}{l}\boldsymbol{v} \\
-\end{array}$ & $\begin{array}{l}\psi \\
-\end{array}$ & $\begin{array}{c}e_{0} \\
- \\
\end{array}$ & $\begin{array}{c}\lambda_{0} \\
- \\
\end{array}$ & $\begin{array}{l}S \\
-\end{array}$ & $\begin{array}{l}\kappa \\
-\end{array}$ & $\chi_{0}$ & $\xi$ & $\xi_{d}$ & $\alpha_{0}$ & $\begin{array}{l}\mu \\
-\end{array}$ & $\begin{array}{l}\beta \\
-\end{array}$ \\
\hline Trench & 0 to 2 & 16 & 495 & 1.5 & 1.2 & 1.33 & 1.4 & 0.2 & 0.018 & 2.02 & 0.23 & 3.5 & 0.026 & 10 & 10 & 0.2 & 0.5 & 20 & 0.89 \\
\hline Upper-1 & 0 to 5 & 16 & 495 & 1.5 & 1.2 & 1.33 & 1.4 & 0.2 & 0.018 & 2.02 & 0.26 & 3.5 & 0.026 & 11 & 11 & 0.2 & 0.5 & 20 & 0.89 \\
\hline Upper-2 & 5 to 10 & 16 & 200 & 1.5 & 1.2 & 1.33 & 1.4 & 0.2 & 0.022 & 2.02 & 0.3 & 4 & 0.035 & 11 & 11 & 0.2 & 0.5 & 20 & 0.89 \\
\hline Upper-3 & 10 to 15 & 16 & 250 & 1.5 & 1.2 & 1.33 & 1.1 & 0.2 & 0.022 & 2.02 & 0.3 & 4 & 0.035 & 12 & 12 & 0.2 & 0.5 & 20 & 0.89 \\
\hline Upper-4 & 15 to 20 & 16 & 299 & 1.5 & 1.2 & 1.33 & 1.1 & 0.2 & 0.022 & 2.07 & 0.3 & 4 & 0.035 & 12 & 12 & 0.2 & 0.5 & 20 & 0.89 \\
\hline Upper-5 & 20 to 32 & 16 & 383 & 1.5 & 1.1 & 1.33 & 0.8 & 0.2 & 0.018 & 1.70 & 0.24 & 3.5 & 0.035 & 11 & 11 & 0.2 & 0.5 & 20 & 0.89 \\
\hline Lower-1 & 32 to 42 & 19 & 491 & 1.5 & 1.0 & 1.30 & 0.8 & 0.2 & 0.016 & 1.50 & 0.18 & 3 & 0.015 & 10 & 10 & 0.2 & 0.5 & 20 & 0.89 \\
\hline Lower-2 & 42 to 52 & 19 & 589 & 1.5 & 0.9 & 1.30 & 0.8 & 0.2 & 0.016 & 1.30 & 0.16 & 2.5 & 0.015 & 8 & 8 & 0.2 & 0.5 & 20 & 0.89 \\
\hline Lower-3 & 52 to 70 & 19 & 724 & 1.5 & 0.8 & 1.30 & 0.8 & 0.2 & 0.016 & 1.10 & 0.14 & 2.5 & 0.015 & 8 & 8 & 0.2 & 0.5 & 20 & 0.89 \\
\hline
\end{tabular}

Note: the bolded numbers are to highlight the difference in parameter input between MEVP-DS and MEVP family of models (MEVP, EVP+SCLAY1, EVP, and S-CLAY1S). 


\subsection{FOUNDATION AND CREST SETTLEMENT}

\subsubsection{CONSTRUCTION SETTLEMENT OF DAM FOUNDATION}

Figure 5.15 presents the settlement at four elevations observed when construction was completed in November 1975. The tesults demonstrate that the numerical models capture the construction settlement reasonably well. Note that the measurements show some dispersion potentially owing to the construction disturbance. In addition, the compared models appear to yield a rather marginal difference. This result has been expected, since it is the stage when the elastic response of the over consolidated foundation would dominate.

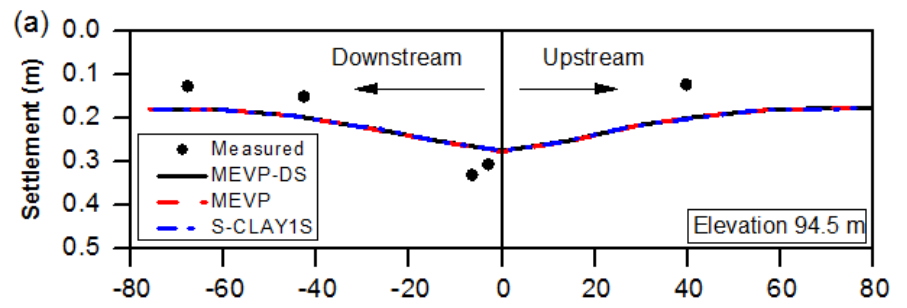

(b)

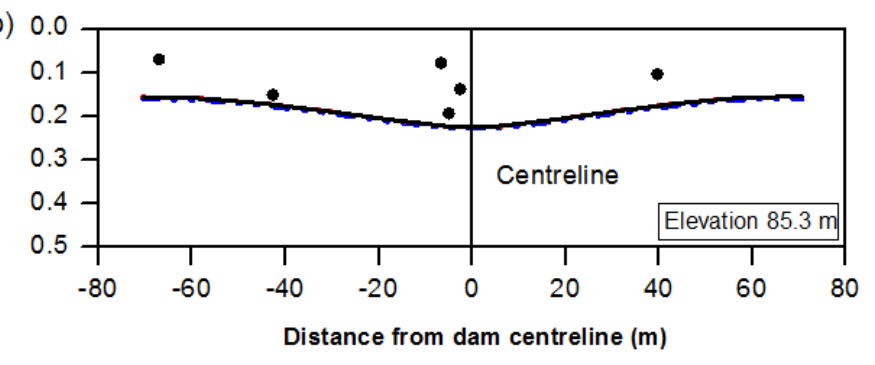

(c)

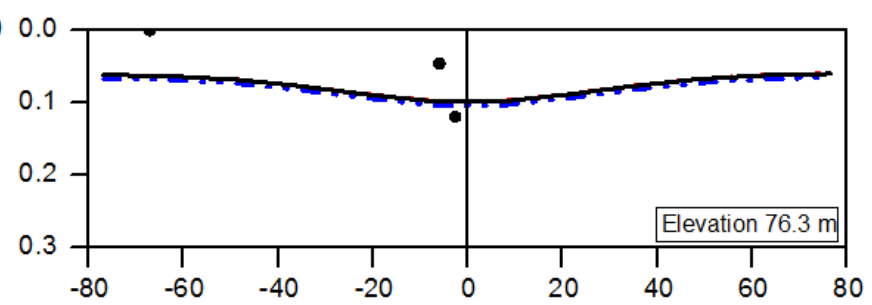

(d)

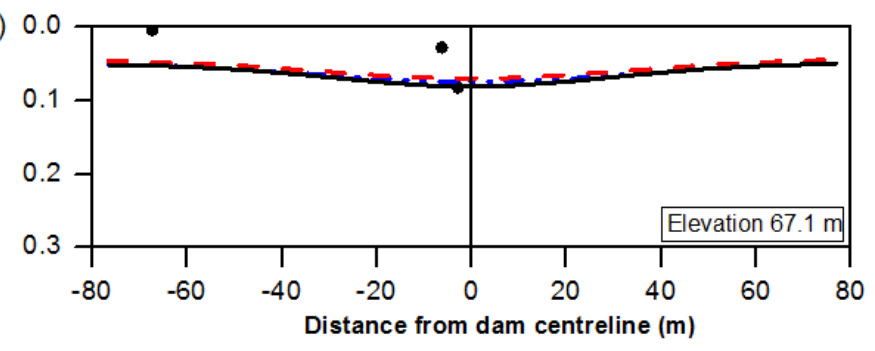

Figure 5.15 Settlement at different elevations due to dam constructions 


\subsubsection{LONG-TERM SETTLEMENT OF DAM CREST}

The long-term crest settlement has been measured by a group of surface monuments (SM) deployed in the longitudinal direction. The teadings from three surface monuments, including SM. 7, SM. 12, and SM. 13, are plotted against the numerical prediction in Figure 5.16 (a). Note that SM. 7 and SM. 12 represent upper and lower boundaries of crest settlements observed in Area 1 region. In addition, SM. 12 and SM. 13 are located in the typical region with the greatest dam height, thus they are expected to provide more comparable reading of model simulations.

The model results demonstrate that MEVP-DS and MEVP simulate the settlement development evidently well. Other models tend to underestimate the settlements with various magnitudes. SCLAY1S provides the least accurate prediction among models used in the FEM analysis.

Figure 5.16 (b) presents the crest settlement development in a logarithmic time scale, showing that the crest settlement is still progressing in a stable manner. However, EVP+S-CLAY1, EVP, and S-CLAY1S predict the settlement to slow down significantly after 2016. However, creep settlements would still slowdown in the future, as creep limits have been demonstrated by Yin et al. (2002).

Furthermore, the result demonstrates also that anisotropy contributes a limited proportion to settlement. This conclusion is based the marginal difference between EVP+S-CLAY1 (0.83 m) and EVP $(0.78 \mathrm{~m})$ predicted in 2016.

The comparison between MEVP-DS, MEVP and S-CLAY1S (Figure 5.17) demonstrates the importance of creep in long-term settlement development. For example, the primary settlement predicted by S-CLAY1S is $0.65 \mathrm{~m}$ in 2016 . This value accounts for $39 \%$ of the total operational settlement $(1.67 \mathrm{~m})$ measured in 2016 . The results imply that the creep settlement may constitute a majority of $61 \%$ of the total settlement, if the difference between S-CLAY1S and MEVP is assumed to be from creep. 

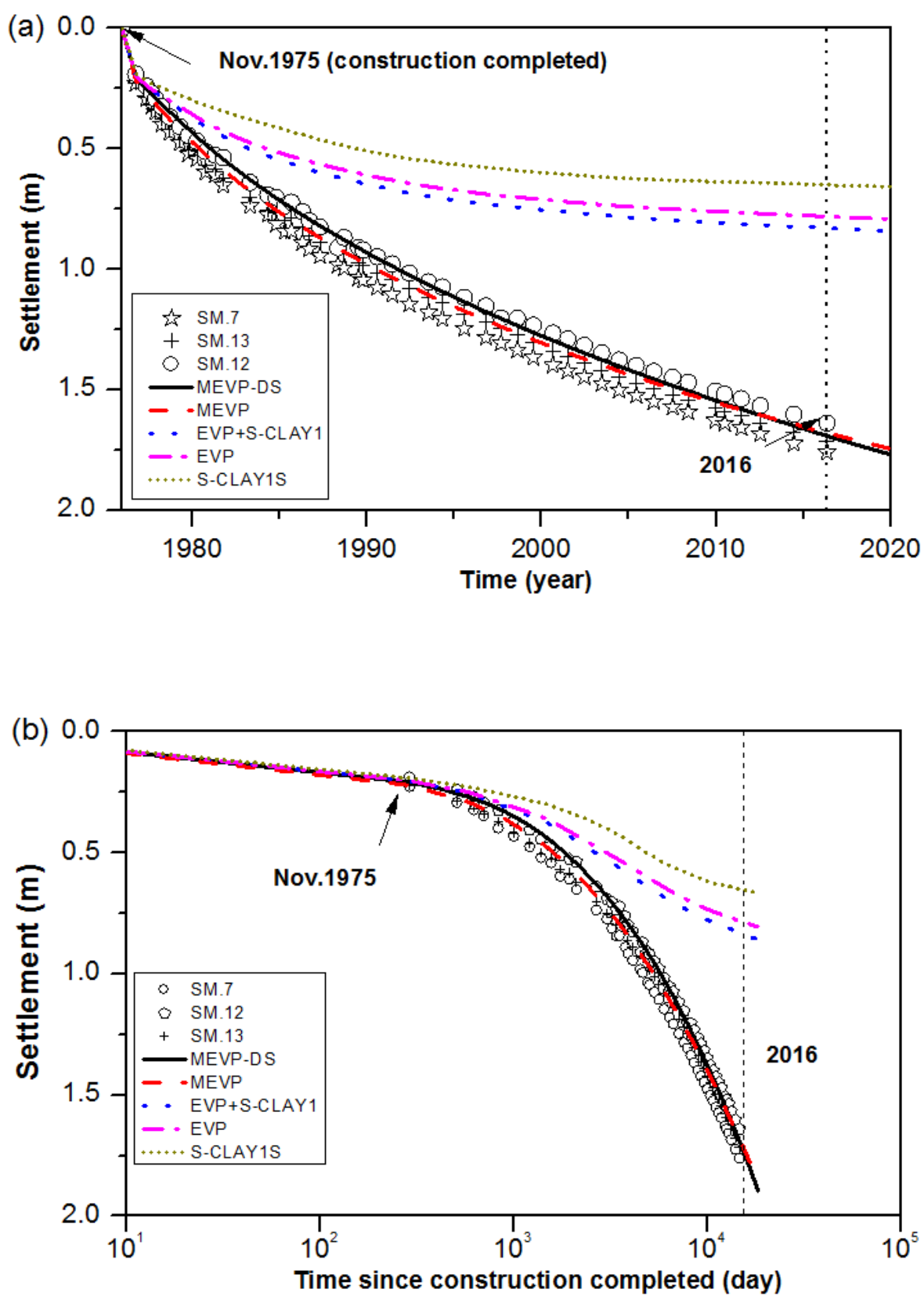

Figure 5.16 Comparison between the measured and predicted crest settlement 


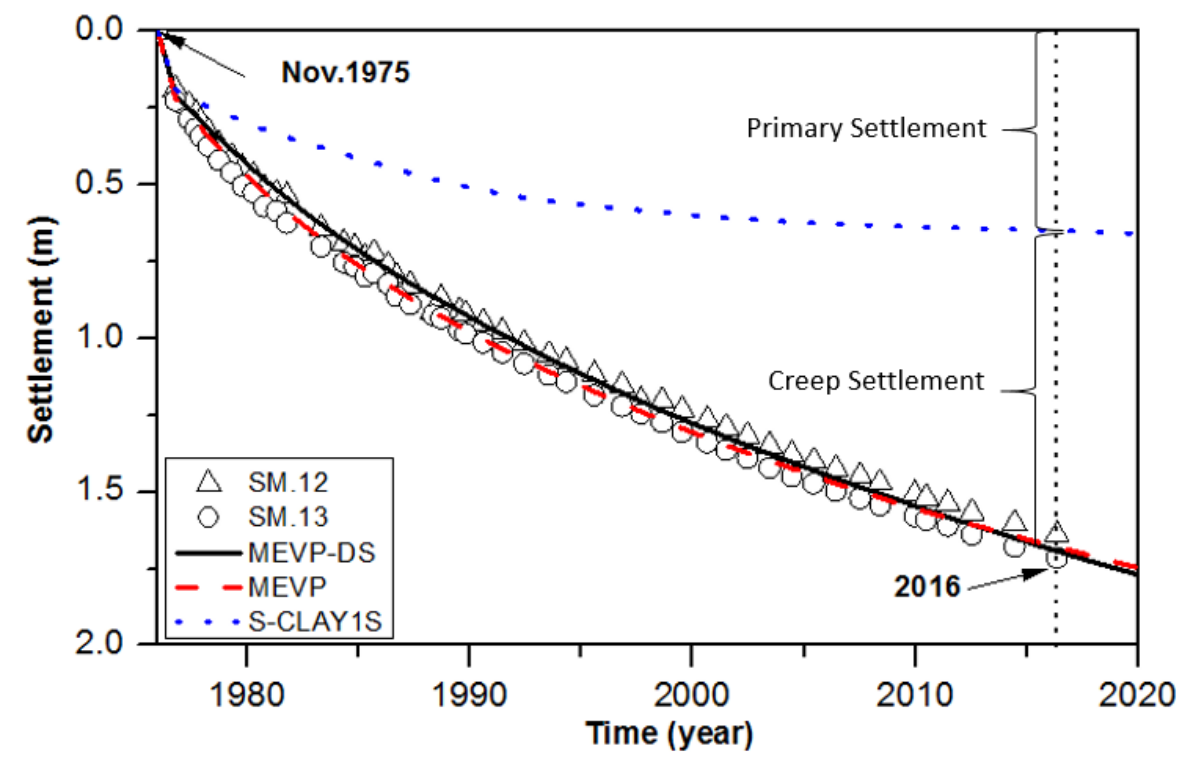

Figure 5.17 Comparison between the settlements predicted by different models

\subsubsection{SIMULATED FOUNDATION SETTLEMENT PROFILE IN SELECTED YEARS}

This section presents the predicted settlement profiles at four different depths below the foundation surface: $0 \mathrm{~m}, 5 \mathrm{~m}, 10 \mathrm{~m}$, and $20 \mathrm{~m}$, as seen in Figure 5.18. The results show that MEVPDS and MEVP appear to yield very close prediction of settlements at the foundation surface, where the dissipation finished almost instantly. However, S-CLAY1S predicts a rather smaller settlement compared to other two models at all depths. On the other hand, MEVP-DS appears to estimate more settlements in deeper areas. This difference reveals that MEVP-DS predicts a deeper extension of the settlement than MEVP. Similar observations can be seen in Figure 5.19, where the foundation settlement as a function of time has been presented. The tracked point of each depth is located along the dam centreline. 

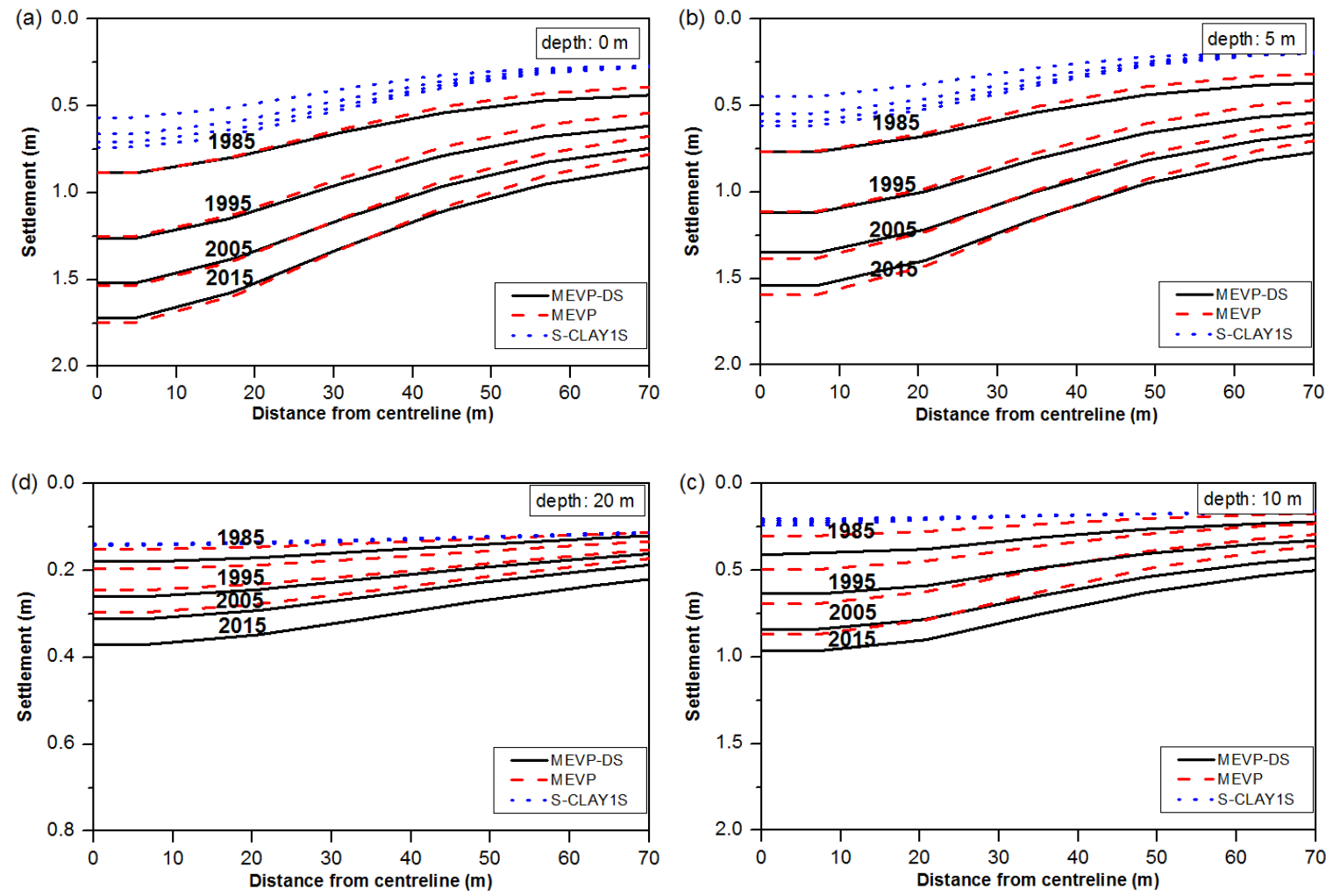

Figure 5.18 Simulated foundation settlement profiles at different depths
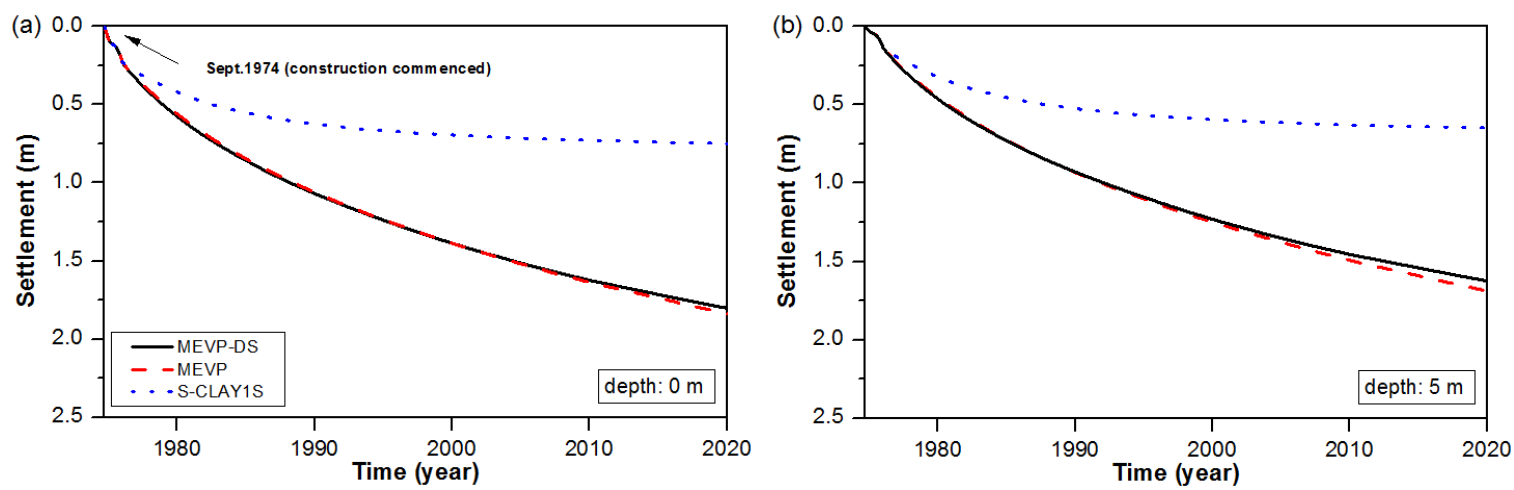

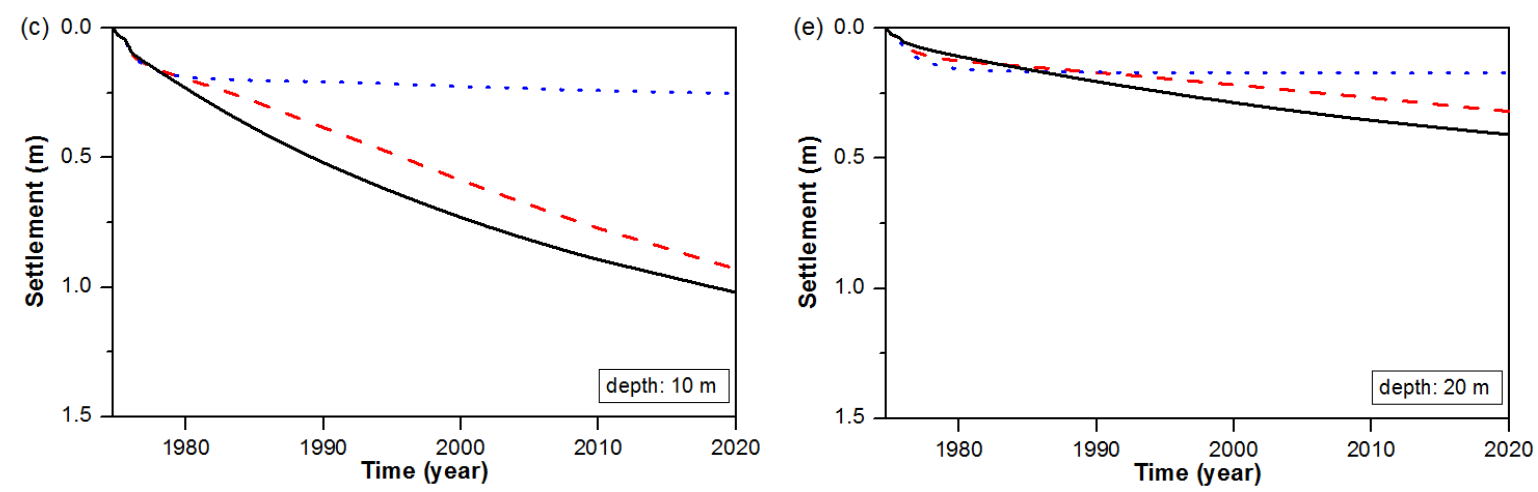

Figure 5.19 Simulated centreline settlement with time at different depths

\subsection{EXCESS PORE PRESSURE IN THE FOUNDATION}

\subsubsection{EXCESS PORE PRESSURE IMMEDIATELY AFTER CONSTRUCTION}

The excess pore pressure $(\Delta u)$ build-up at the end of construction has been captured by piezometers. In the studied Area 1 region, there were 7 piezometers deployed, shown in Figure 5.20. They are grouped into three sections from the upstream to the downstream: Section A (downstream, $56 \mathrm{~m}$ to the centreline), Section B (dam centreline), and Section C (upstream).

Piezometer readings in November 1975 are shown in Figure 5.21 together with model predictions. A rather negligible difference can be seen between the prediction by MEVP-DS and MEVP. In addition, numerical results agreed well with the observation at all sections inspected. It is indicated that the maximum $\Delta u$ is approximately at $20 \mathrm{~m}$ below the foundation surface, when the embankment construction was just completed.

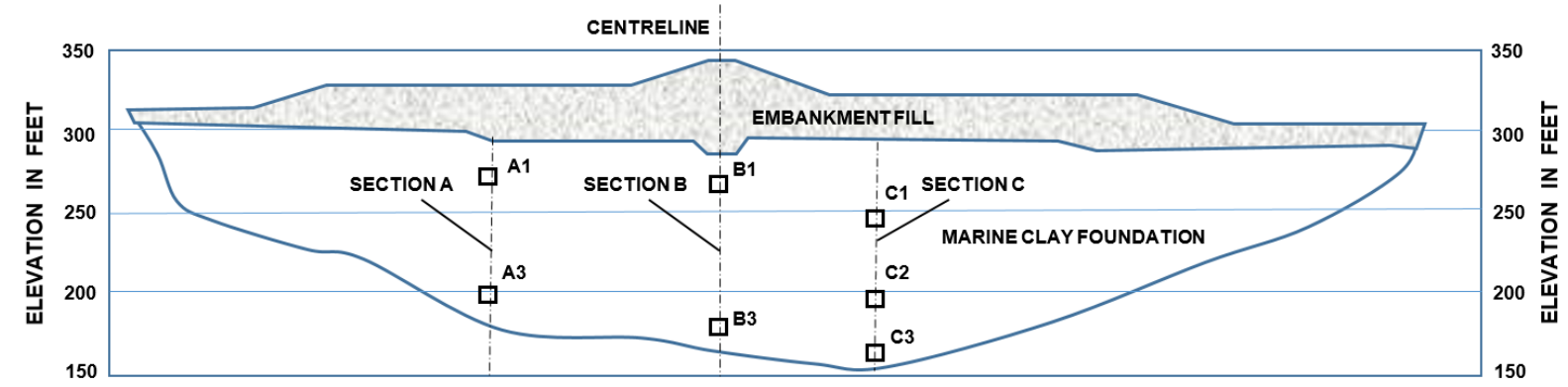


Figure 5.20 Piezometer instrumentation in Area 1 region

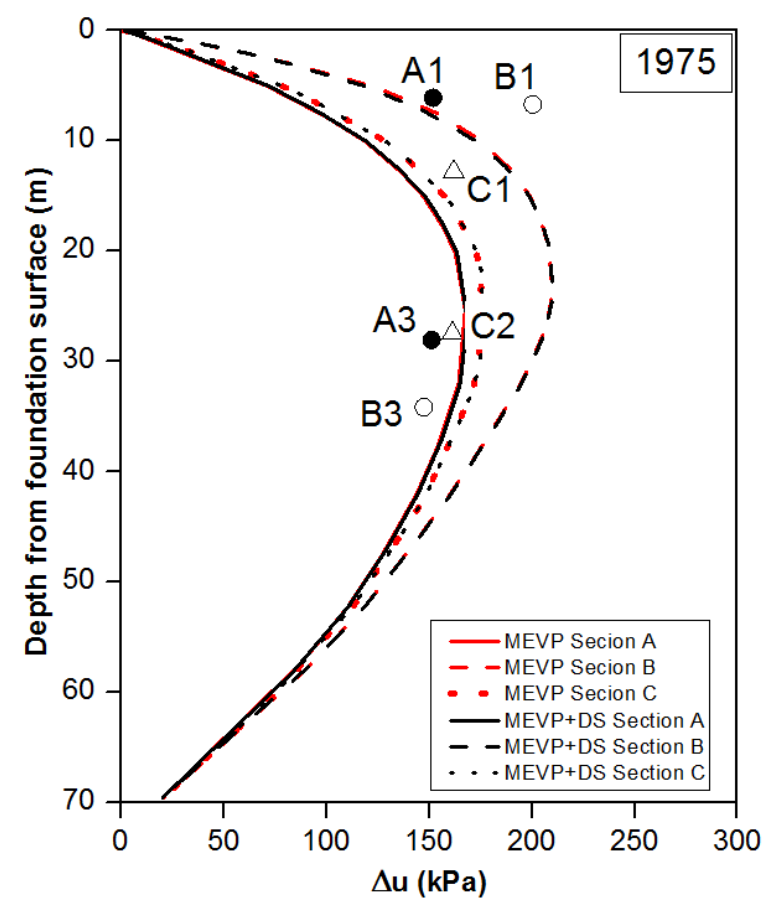

Figure 5.21 Predicted and measured excess pore pressure in 1976

\subsubsection{EXCESS PORE PRESSURE IN THE YEAR OF 2015}

Most of the piezometers installed in 1976 are observed to have malfunctioned over time. This could be primarily due to aging, and thus the recorded pore pressure readings are not considered reliable. For that reason, a new investigation program has been initiated in 2015. In this program, six new vibrating wire piezometers (VWPZ) were placed in two boreholes where intact soil samples were retrieved, three piezometers in each borehole.

The new piezometer readings in 2015 have been interpreted assuming the groundwater table to be at the foundation surface (EL $88.4 \mathrm{~m}$ ), as specified as Interpretation B in Figure 5.22. However, it is also argued that the actual water table might be at a higher level of $2 \mathrm{~m}$ below the berm surface (EL $95.4 \mathrm{~m}$ ). This has resulted in a lower interpretation of $\Delta u$ - Interpretation A. 


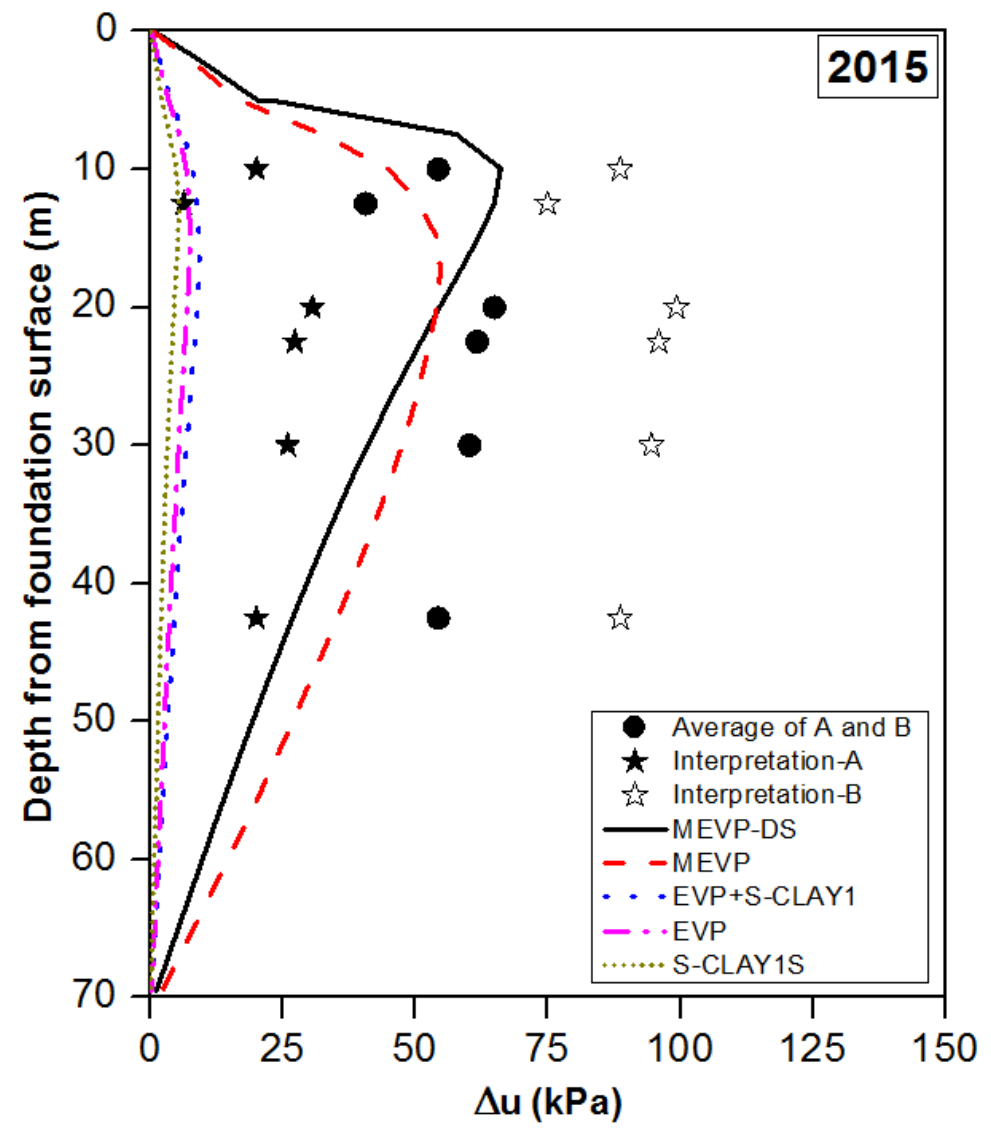

Figure 5.22 Excess pore pressure distribution with depth along the dam centreline in 2015

For the numerical simulation, it is noticed that MEVP-DS and MEVP provide a more accurate prediction of $\Delta u$ compared to other models. MEVP-DS predicts a higher $\Delta u$ level at shallow depths, while MEVP estimates more $\Delta u$ in the deeper area.

The result also demonstrates that the creep behaviour of foundation, in combination with destructuration, delays the dissipation process (Indraratna et al. 1994, Chai and Miura 1999, Chai et al. 2018). In some cases (Leroueil and Roy 1978, Karim et al. 2013), the $\Delta u$ has been observed to rise after construction owing to softening of sensitive marine clay.

\subsubsection{SIMULATED $\triangle U$ DISSIPATION OF CENTRELINE SECTIONS}

This section aims to further investigate the model difference when predicting $\Delta u$. Figure 5.23 shows the predicted dissipation in the upper clay region, including $5 \mathrm{~m}, 10 \mathrm{~m}, 20 \mathrm{~m}$, and $32 \mathrm{~m}$ 
depth. The results demonstrate creep results in a significant delay in dissipation, if one compares MEVP-DS and MEVP with S-CLAY1S.

MEVP-DS appears to yield a slower dissipation process at the $10 \mathrm{~m}$ depth than MEVP. In the deeper area, MEVP predicts the dissipation to be slower. However, the results by two models become overlapped in 2020 .

Figure 5.24 plots the dissipation with logarithmic time. Another axis $u / u_{\max }$ is added to help evaluate the progress of primary consolidation. It has been pointed that $u / u_{\max }$ is not equivalent to the consolidation degree $U$ for soft soils which are prone to creep. This is because in these soils primary settlements progresses way ahead of dissipation (Krenn 2008).

The results show that MEVP-DS and MEVP share the similarity in predicting a slump of $\Delta u$ right after the construction. This could be due to the early elastic compression stage when the soil is stiff and thus very fast consolidation can mobilize.

In addition, MEVP-DS and MEVP estimate that the dissipation will be still ongoing in the deeper region of upper clays for a considerable amount of time passing 2100. 

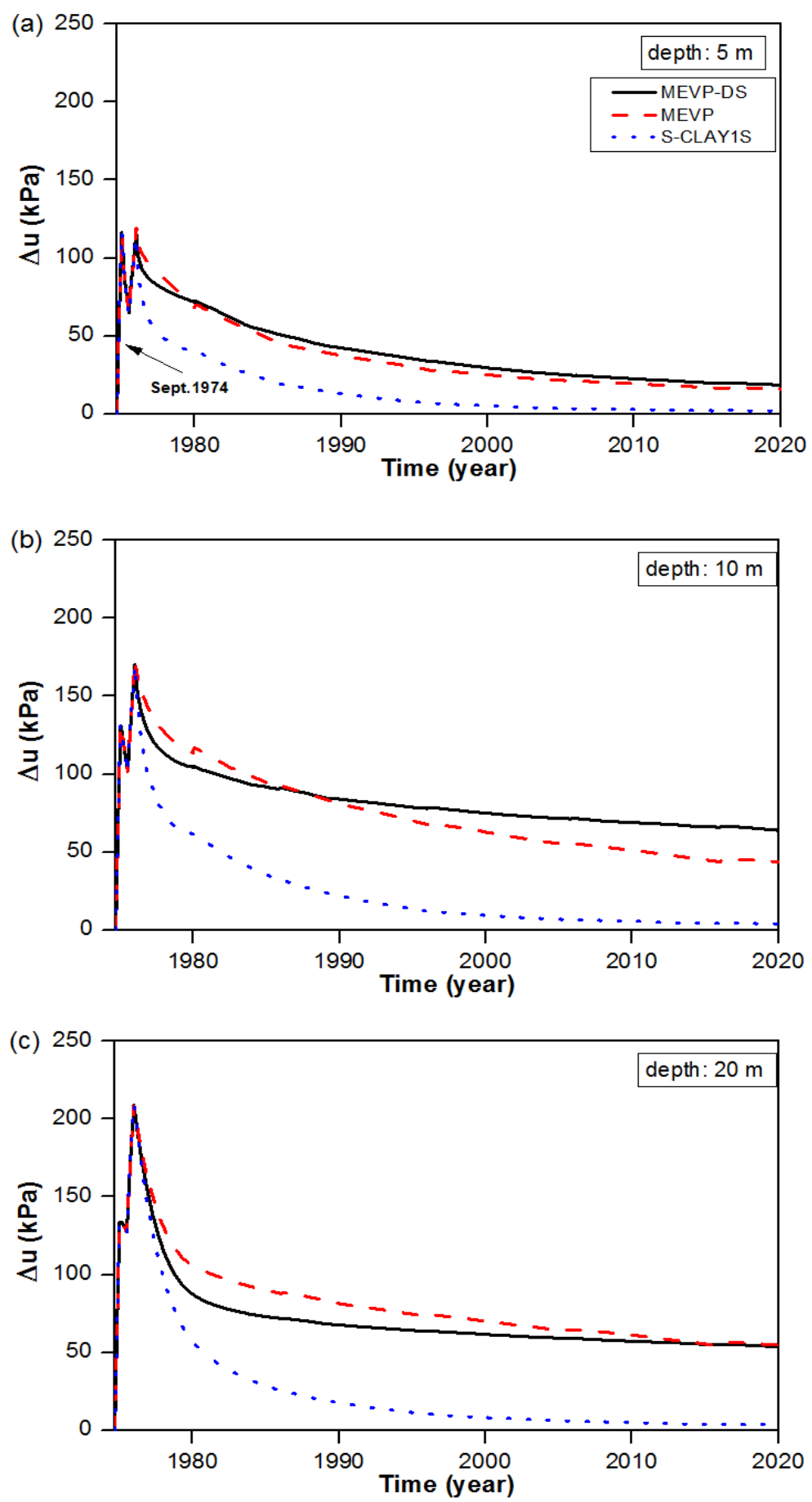

Figure 5.23 Simulated $\Delta u$ dissipation at foundation centerline 

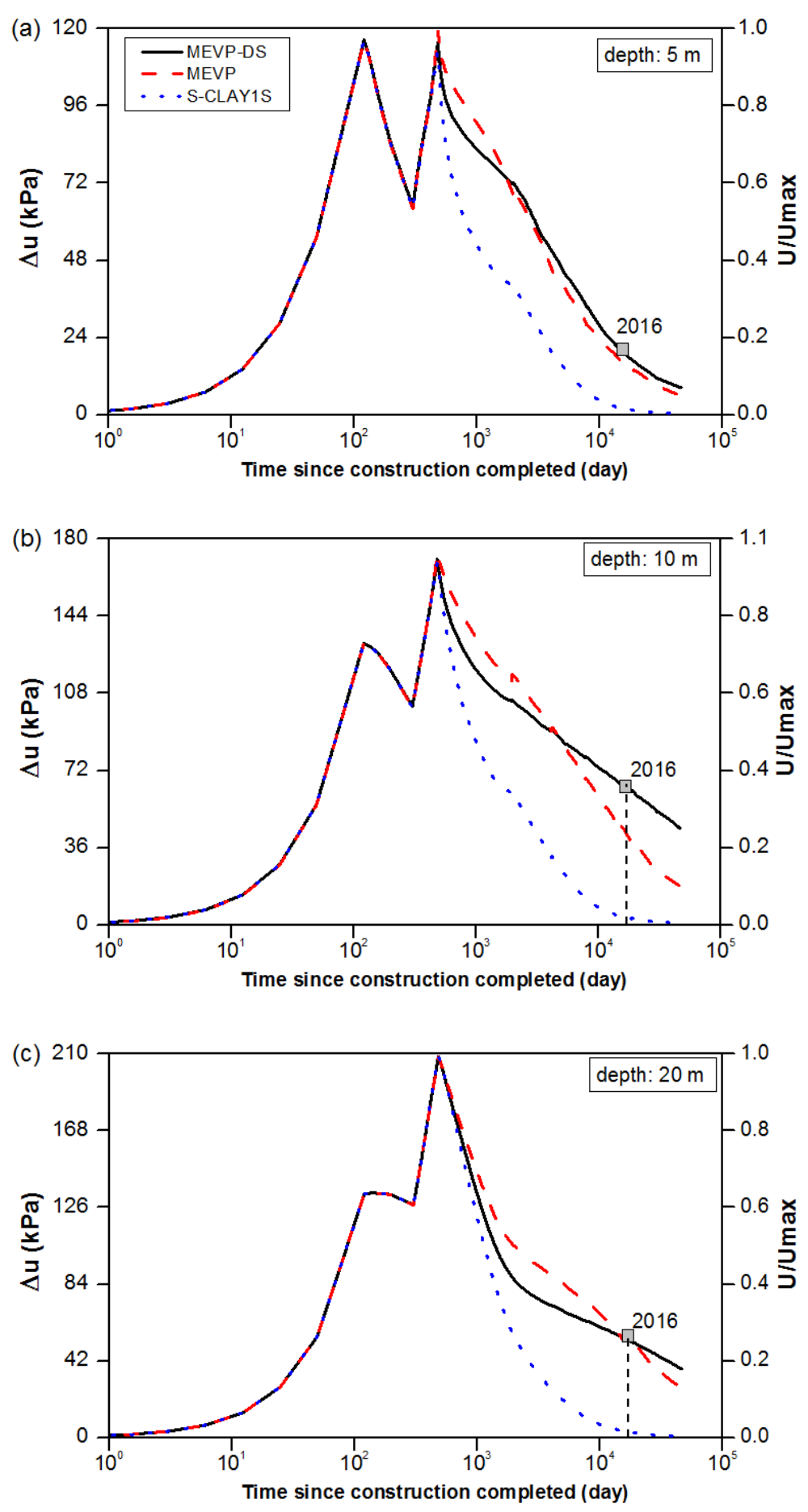

Figure 5.24 Simulated $\Delta \mathrm{u}$ dissipation at foundation centreline plotted in log scale 


\subsection{LATERAL SPREADING IN THE FOUNDATION}

\subsubsection{LATERAL SPREADING AT IS-2 IN 1979 AND 2008}

The lateral spreading $\left(U_{x}\right)$ inside foundation has been monitored by slope inclinometers installed since 1975. The reading of Slope Indicator No.2 (SI-2) is studied here for comparison with numerical prediction. Other gauges are not used here as being too far from the dam toe area where the maximum lateral spreading occurs. However, it still should be mentioned that even the SI-2 might not provide the maximum spreading reading, since it is still located $6 \mathrm{~m}$ away from the dam toe.

$U_{x}$ recorded in 1979 and 2008 are compared with the predictions in Figure 5.25. The model results demonstrate that MEVP-DS and MEVP provide a better prediction in both years, in contrast to the rest of soil models.

In addition, MEVP-DS appears to match the 2008 readings slightly better than MEVP by $9 \%$. Nevertheless, all models used in this analysis have shown to under-predict the maximum lateral spreading, $U_{\max }$, in 2008 by $30 \%$ to $72 \%$.

It is also of interests to normalize the spreading using $u_{x} / u_{\max }$, and the results can be found in Figure 5.26. $u_{x} / u_{\max }$ is the maximum spreading occurring at $5 \mathrm{~m}$ below the foundation surface. MEVP-DS and MEVP match with the measurement in 1979 in a satisfactory way, except SCLAY1S which shows some discrepancy between $10 \mathrm{~m}$ and $20 \mathrm{~m}$ depth.

Figure 5.27 is to investigate $u_{\max }$ development related to crest settlement, $s$. It is achieved by calculating the ratio $u_{\max } / s$ as the function of time. It has been observed that $u_{\max } / s$ was stabilized at 0.15 after 1985 . Note that the $u_{\max }$ measured by SI-2 is not necessary the maximum spreading, which has been reported to occur under the embankment toe (Tavenas et al. 1979, Kelly et al. 2018, Rezania et al. 2018). Therefore, a more precise $u_{\max } / s$ ratio could be higher than 0.15 , bringing the value closer to the empirical 0.18 as suggested in (Tavenas et al. 1979).

Except MEVP-DS, other models predict a downward trend of $u_{\max } / s$ after reaching the peak in 1980. This indicates that the creep settlement proceeds in these cases in a faster way than spreading. On the other hand, MEVP-DS has predicted a more stabilized $u_{\max } / s$ ratio, which is close to the observation. 

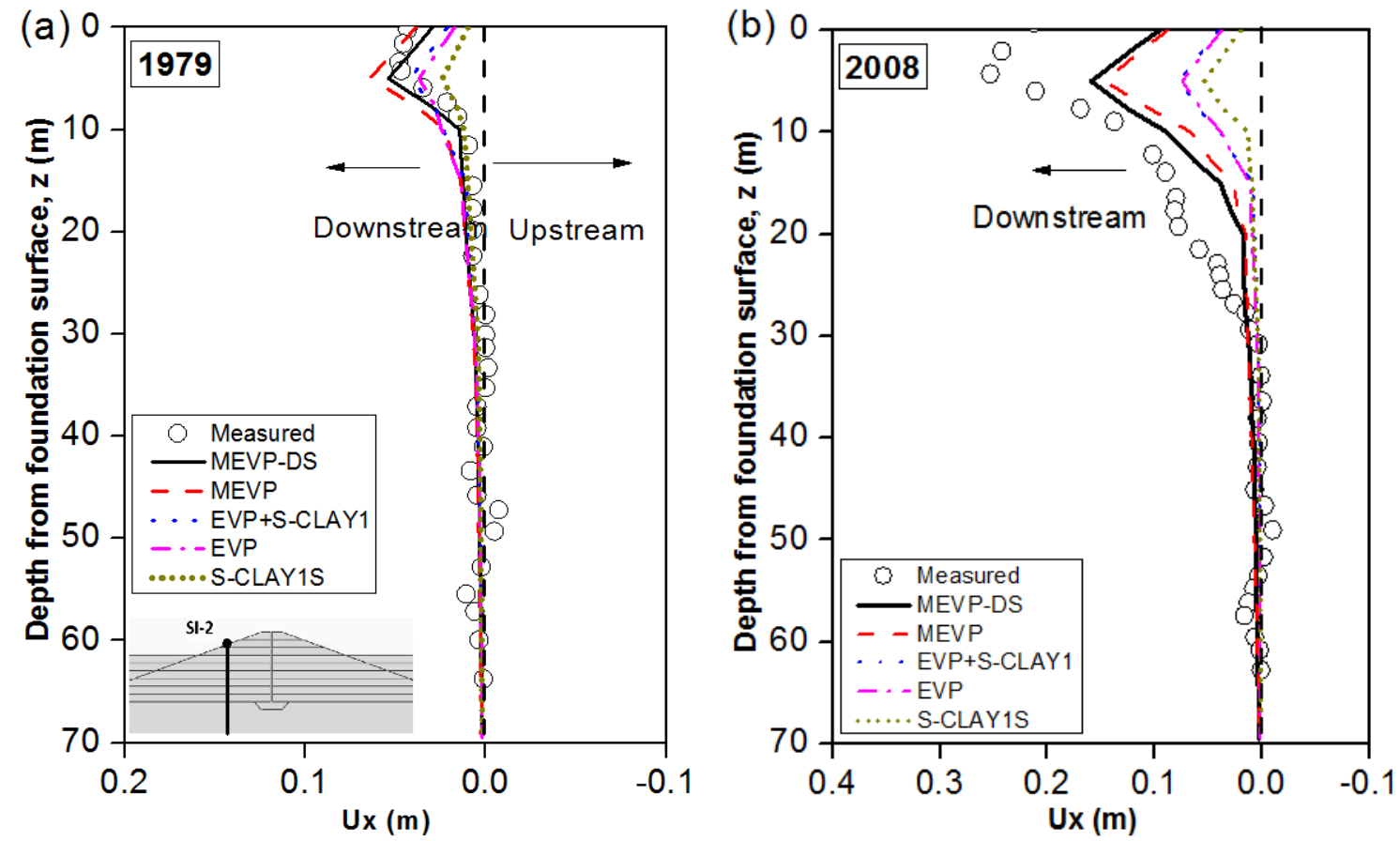

Figure 5.25 Lateral spreading of SI-2 compared with numerical prediction
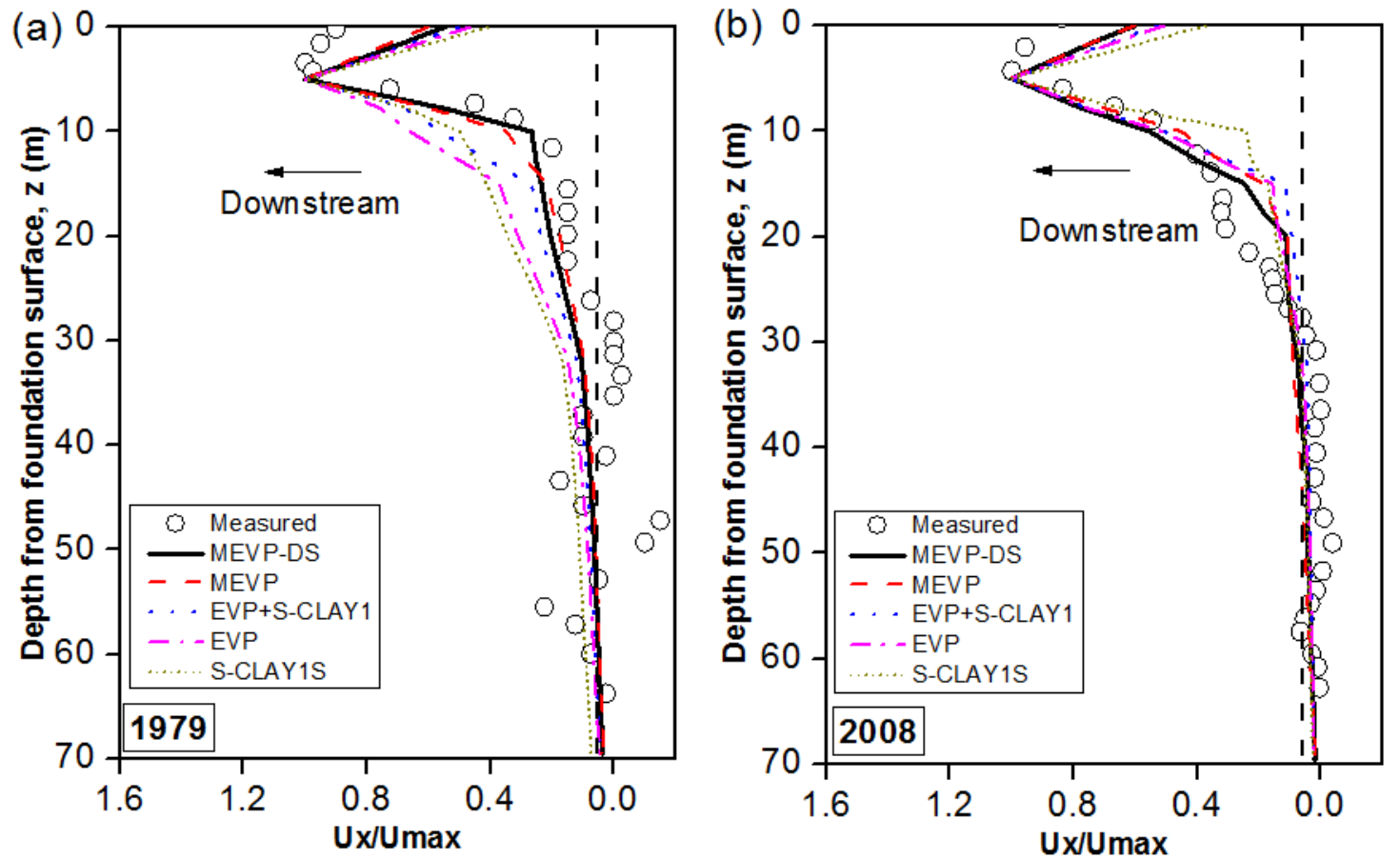

Figure 5.26 Normalized lateral spreading of SI-2 compared with numerical prediction 


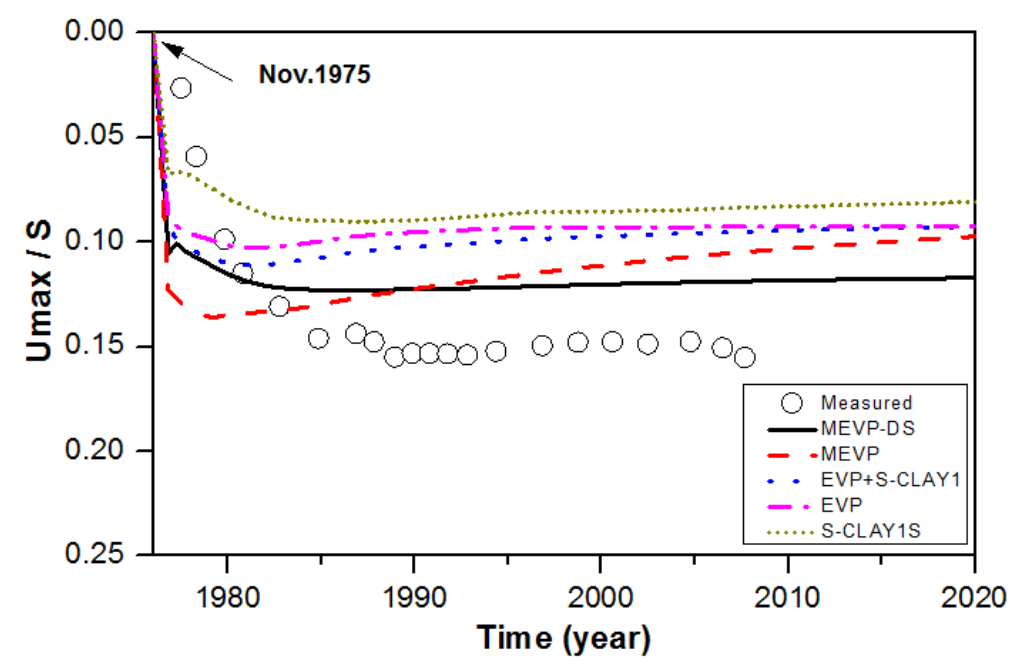

Figure $5.27 \mathrm{u}_{\max } / \mathrm{s}$ ratio as a function of time

\subsubsection{SIMULATED $U_{x}$ COMPARED AT 5 M DEPTH}

It is known from the previous section that $u_{\max }$ captured by IS-2 occurs at $5 \mathrm{~m}$ depth below the foundation surface. This section aims to investigate the profile of $u_{x}$ and its evolution with time. The simulated results are presented in Figure 5.28.

First of all, the simulation confirms that the real $u_{\max }$ is under the embankment toe. Secondly, the MEVP-DS and MEVP predict similar result before 2005. After that, the difference starts to manifest that MEVP-DS predicts more lateral spreading than MEVP. On the other hand, the $u_{x}$ profile by S-CLAY1S has been almost unchanged since 2005.
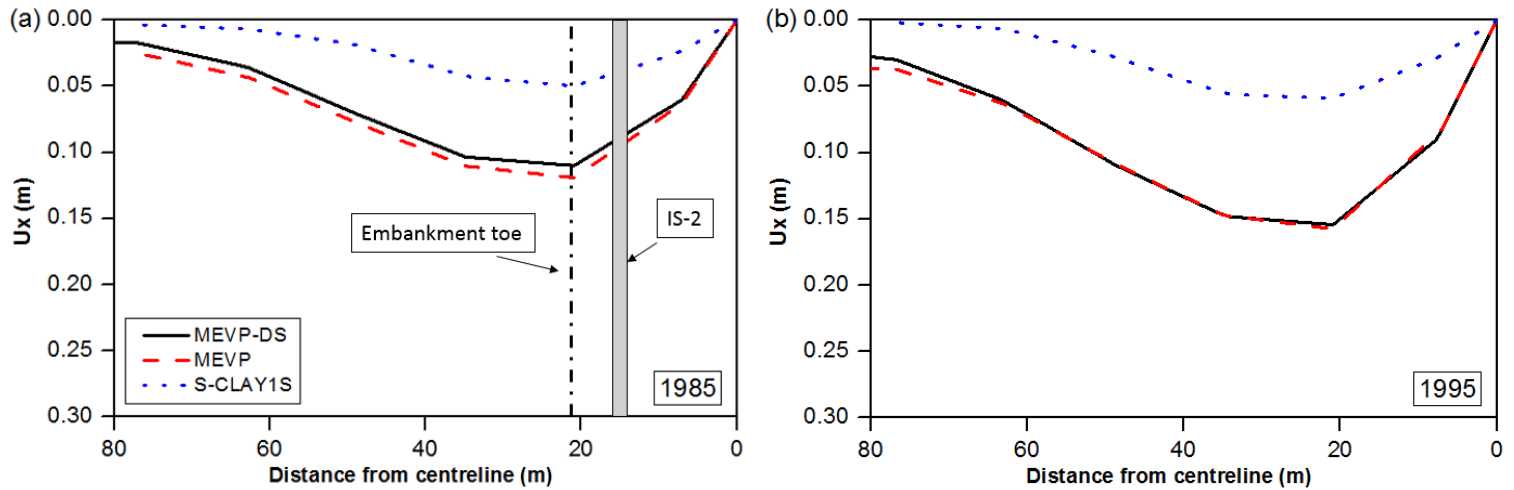

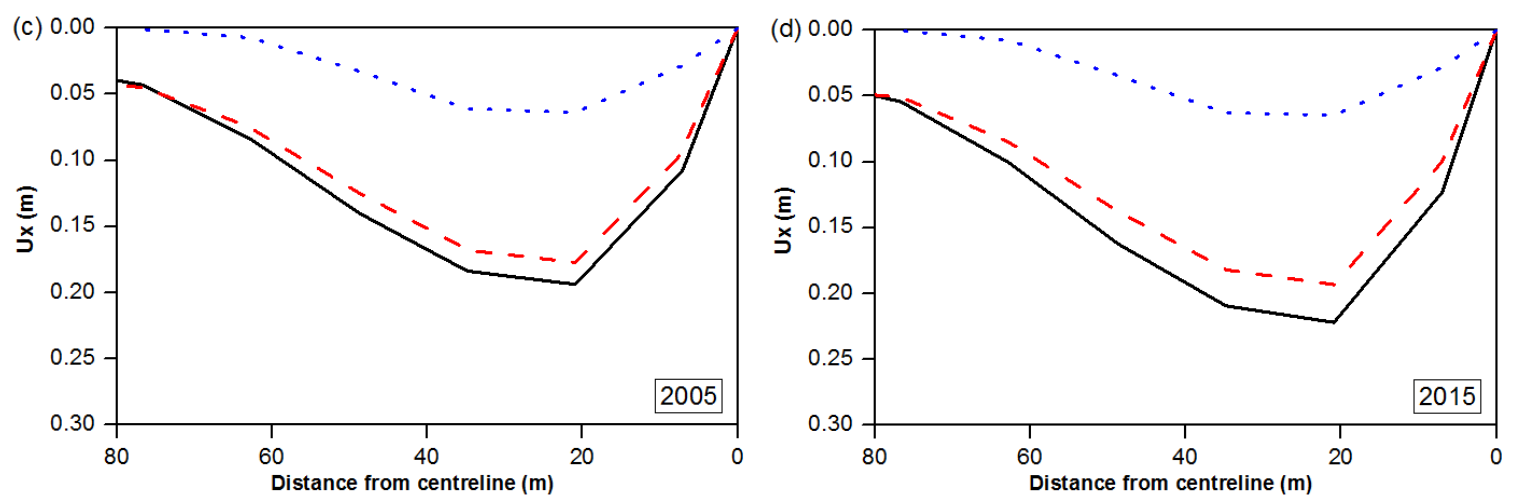

Figure 5.28 Simulated development of $U_{x}$ at $5 \mathrm{~m}$ depth with time

\subsection{CONCLUSIONS}

This chapter presents a further verification of MEVP-DS in simulating the long-term settlement of an embankment on sensitive Champlain Sea clay - Waba dam. The field monitoring data collected over the course of forty years is used to evaluate the performance of the soil models. Following conclusions can be drawn from the simulation results.

1. The simulation indicates that the consideration of deviatoric softening is beneficial to some aspects of the embankment simulation, especially the prediction of $U_{x}$.

2. Consideration of creep is paramount for the Waba dam simulation. It is shown that creep not only affects the long-term settlement prediction, but also reproduces the delayed $\Delta u$ dissipation. In addition, creep has been found to influence $U_{x}$ in a noticeable manner as well.

3. MEVP-DS and MEVP yield almost the same prediction during the construction stage of the dam. However, the differences between two models start to become more noticeably over time.

4. MEVP-DS predicts the foundation settlement to extend deeper than MEVP.

5. MEVP-DS predicts a slower dissipation than MEVP within the top $10 \mathrm{~m}$ depth, while MEVP suggests a slower dissipation in the deeper area.

Some limitations of this case study can be concluded. These limitations can be attributed to MEVP-DS or the 2-D embankment model itself: 
1. Even though MEVP-DS appears to work better than other models in predicting $U_{x}$, the overall underestimation by MEVP-DS is still about 30\%. There might be some underestimated lateral creep occurring in the foundation.

2. Some simulation differences between MEVP-DS and MEVP could be due to the destructuration parameter inputs, as two models have been calibrated independently. Different definitions of the compression indices in MEVP-DS and MEVP, along with the consideration of $\varsigma$ in MEVP-DS, lead to the difference in the calibrated destructuration parameters.

3. This study models a typical cross-section of the embankment. However, the actual foundation surface is not smoothly flat. In addition, the embankment height and berm thickness are also different in other sections. Hence, 3-D modeling of the dam would help explain the discrepancy of model simulations. 


\section{SUMMARY AND CONCLUSIONS}

\subsection{INTRODUCTION}

This research investigates the influence of plastic deviatoric strains on the compressibility of soft clays. A number of multi-stage incremental anisotropic consolidation tests performed on Finnish soft clays reveal that Finnish clay appears to be more compressible when subject to high stress ratios than to low stress ratios. Therefore, the plastic deviatoric strains induced by stress ratios are assumed to contribute to yield surface softening in soft clays. A relationship between soil compressibility and stress ratio is established based on the published test data. Based on this relationship, a modified deviatoric softening law for soft clays is introduced, where only one additional parameter, $\varsigma$, is introduced to govern the contribution of plastic deviatoric strains to yield surface softening.

A new model called MEVP-DS has incorporated this softening law and implemented in the Yin's elasto-viscoplastic framework to be able to consider the soil features, such as viscosity, yield surface anisotropy, and destructuration. MEVP-DS has been programmed by FORTRAN and implemented in Plaxis V8.2.

The verification of MEVP-DS has been done through three phases. Phase I is to verify the model through simulating seven published incremental anisotropic consolidation tests on intact Finnish clays. This phase aims to verify the combination of deviatoric softening and yield surface anisotropy feature of the model. Destructuration and creep have been ignored in this phase. The model prediction of MEVP-DS has been compared with S-CLAY1 and MCC to demonstrate its capacity. Phase II is to verify the model by simulating the 1-D consolidation tests on undisturbed sensitive Champlain Sea clay. The simulated tests include seven constant rate-of-strain consolidation tests, two conventional oedometer consolidation tests, and three 1-D creep tests. Intact soil samples used in the study were retrieved from the deposit of Waba dam in Eastern Canada. This phase of model simulation aims to verify all features of MEVP-DS model. Phase III is to verify the model through a real case by simulating the long-term settlement of an embankment dam, Waba Dam, on Champlain Sea clay foundation. The dam was built on top of a $70 \mathrm{~m}$ thick deposit of Champlain Sea clay and has accumulated over $1.6 \mathrm{~m}$ settlement since its construction 
completed in 1975. In this study, a 2-D plain strain model of a typical cross section of Waba dam has been established. The numerical predictions of MEVP-DS are compared with 40 year field monitoring data.

\subsection{MAIN CONCLUSIONS}

1. The simulations of incremental anisotropic consolidation tests on Finnish clays demonstrate that MEVP-DS provides noticeable improvements to both volumetric and deviatoric strain prediction in contrast to S-CLAY1 and MCC models. MEVP-DS is proven to be able to capture different compressibility of Finnish clays subject to different stress ratios.

2. MEVP-DS is able to predict the compression behavior of sensitive Champlain Sea clay fairly well. Considering deviatoric softening can be beneficial to the excess pore pressure prediction of constant rate of strain tests on Champlain Sea clay. It is also found that excess pore pressures are highly dependent on soil compressibility, while hydraulic gradients appear to be more related to permeability. Moreover, some improvements are seen in conventional oedometer tests and creep tests simulation using MEVP-DS as well.

3. Viscosity is not a dominant factor for modeling the soil compressibility exhibited during CRS tests. Anisotropy does not appear to be influential either in this study, because the yield surface rotation during the test is rather limited based on assumed initial state parameters.

4. MEVP-DS suggests strain rates lower than $1.5 \% / \mathrm{hr}$ are applicable for the studied Champlain Sea clay to satisfy the ASTM standard of excess pore pressure ratio between 0.03 and 0.15 . For other Champlain Sea clay with less compressibility, the allowable strain rate could be higher.

5. MEVP-DS predicts reasonably well the long-term settlement prediction of Waba dam over the course of 40 years. The consideration of deviatoric softening is shown to improve also other aspects of the simulation, especially the lateral spreading.

6. MEVP-DS yields almost the same construction behavior as other soil models used in the analysis. However, the impact of deviatoric softening on the settlement of the dam 
becomes more noticeably over time. MEVP-DS predicts a slower dissipation than MEVP within the top $10 \mathrm{~m}$ depth, whereas MEVP suggests a slower dissipation in the deeper area.

7. MEVP-DS predicts the foundation settlement to extend deeper into the foundation than MEVP. However, the foundation settlement predicted by MEVP appears to be concentrated more in the shallow layers.

8. Creep is proven to be paramount to Waba dam simulation. It is shown that creep not only influences long-term settlement predictions, but also leads to delayed dissipations. In addition, creep has been found to influence lateral spreading in a noticeable manner as well.

\subsection{SIGNIFICANCE OF THIS RESEARCH}

This dissertation has proposed a modified model which aims to improve the simulation of soil compressibility of soft clays. It is achieved by the proposal of a modified yield surface deviatoric softening law, which describes plastic deviatoric strains contribute to yield surface size softening. The new model, named MEVP-DS, has been shown to provide the improved estimation of soft clay's compressibility subject to 1-D, anisotropy, and more sophisticated loading conditions.

\subsection{FUTURE RESEARCH}

This research attempts to investigate the influence of plastic deviatoric strains on yield surface size softening. Although the new deviatoric softening law has shown some improvements to soft clay simulation, it is acknowledged that the accurate modeling of soft clay compressibility remains a challenging issue. The following summarizes some interesting aspects that deserve further study.

1. For MEVP-DS and MEVP, the incorporation of S-CLAY1S destructuration theory into Bjerrum's timeline framework still requires further investigation. The timeline framework depicts a family of parallel compression lines corresponding to various equivalent creep time. However, when destructuration and deviatoric softening attributed to plastic deviatoric strains are considered, the description of these timelines becomes challenging and is worth further investigation. 
2. The current deviatoric softening law may result in some significant magnitudes of softening when a soil is consolidated close to the critical state ratio. A threshold ratio of the compressibility index at critical state to isotropic state can be considered to better describe these extreme cases. Based on the collected data so far from Finnish clay, this threshold ratio could be 3.35 , while the value for various soils still deserves further study by laboratory evidence.

3. The estimation of $\lambda_{0}$ of MEVP-DS model is highly dependent on the review of IAC tests on Finnish clay, Italian clay, and Champlain Sea clay. More tests on other types of soil, both from remolded and intact samples, remain necessary.

4. Simulating impounding and seepage at Waba dam upstream will add some merits to the model prediction. Some trial models of Waba dam indicate that impounding may result in a less settlement prediction compared to the current model. However, due to the impervious nature of the deposit, it will take a considerable amount of time for the impounded water table to merge with the existing ground water table. The correct simulation of this behavior can be challenging but beneficial. 


\section{APPENDIX A - CODE OF SUBROUTINE (EVP.for)}

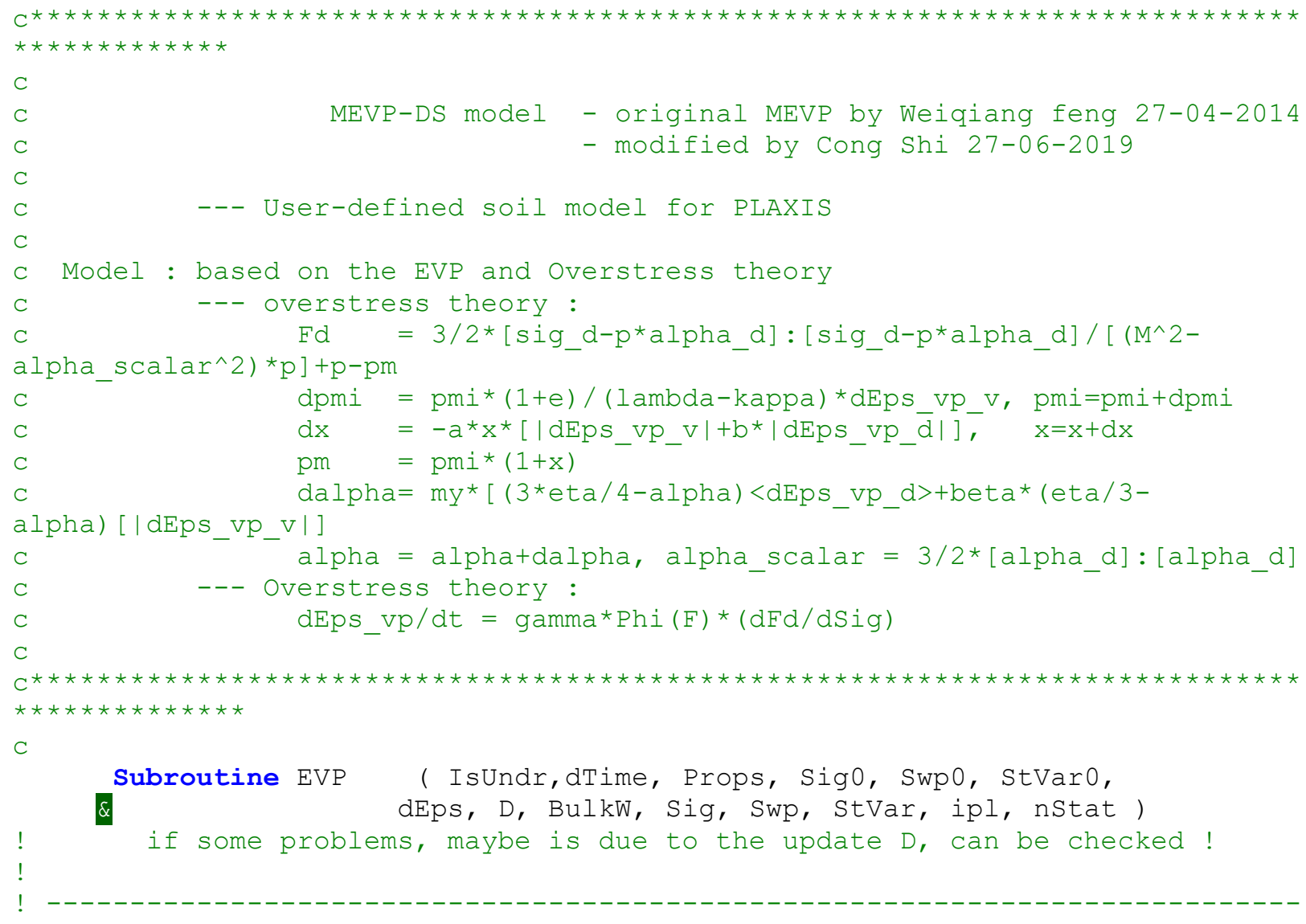

User-defined soil model: EVP-MCC (iMod=1)

Depending on IDTask, 1 : Initialize state variables

2 : calculate stresses

3 : calculate material stiffness matrix

4 : return number of state variables

5 : inquire matrix properties

6 : calculate elastic material stiffness matrix

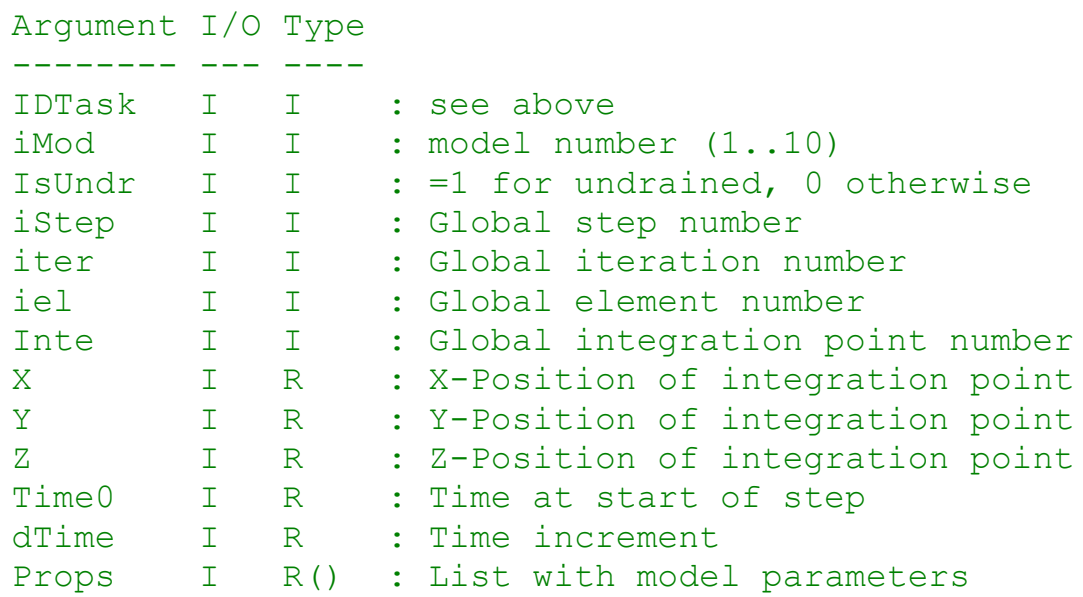




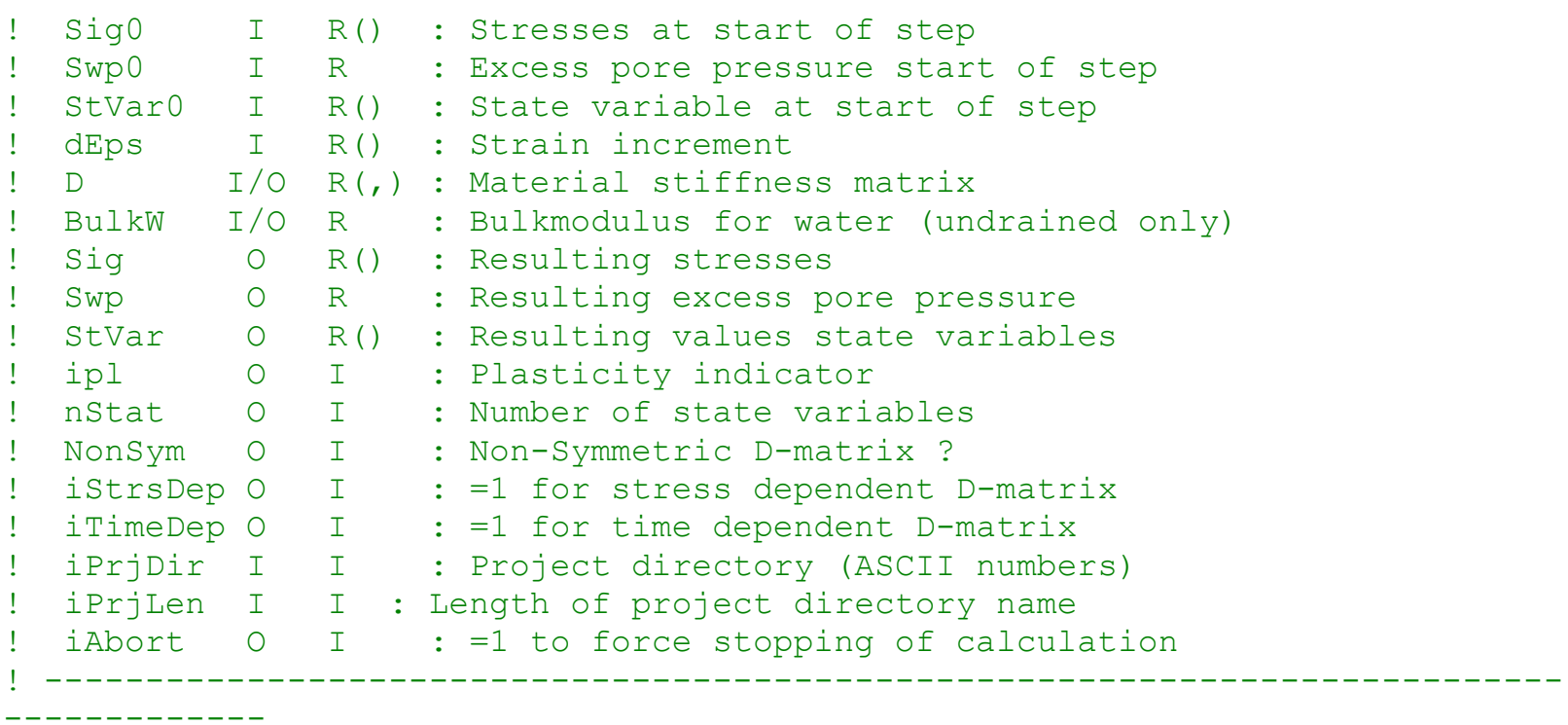

Implicit Double Precision (A-H, O-Z)

\begin{tabular}{|c|c|c|}
\hline Double & Precision & StVar0 (nstat), StVar (nstat) \\
\hline Double & Precision & Sig0(6), dsig(6), Sig(6), sig_d(6) \\
\hline Double & Precision & dEps_vp $(6)$, dEps el $(\bar{\sigma})$ \\
\hline Double & Precision & $D(6,6)$ \\
\hline Double & Precision & Props $(50)$ \\
\hline Double & Precision & $\begin{array}{l}\operatorname{hh}(6,6), \operatorname{hh} 1(6,6), \operatorname{hh} 2(6,6), \operatorname{hh} 3(6,6), \operatorname{hh} 4(6,6), \\
\operatorname{hh} 5(6,6), \operatorname{hh} 6(6,6), \operatorname{hh} 7(6), \operatorname{hh} 8(6,6), \operatorname{htte}(6)\end{array}$ \\
\hline Double & Precision & dF_dSig $(6), \operatorname{Mii}(6,6), \operatorname{VID}(6), \operatorname{dGdSig}(6,6)$ \\
\hline Double & Precision & $\operatorname{ht}(6,6), \operatorname{hd}(6,6), \operatorname{ht1}(6), \operatorname{ht2}(6), \operatorname{hdd}(6,6), \operatorname{ht}(6)$ \\
\hline Double & Precision & dEps0_trial (6), dEps0_vp (6), Sig_de (6) \\
\hline Double & Precision & Sig triale(6), Sig0 trial (6), GG (6), QQ (6) \\
\hline Double & Precision & dddSig_trial (6) \\
\hline Double & Precision & ! Viscosity index \\
\hline Double & Precision & ! Viscosity coefficient \\
\hline Double & Precision & ! Size of dynamic yield surface \\
\hline Double & Precision & ! Calculation index \\
\hline
\end{tabular}

0 :explicit, 1:implicit,0.5:demi-implicit

\begin{tabular}{|c|c|c|c|c|c|c|}
\hline DATA & $\mathrm{VID} / 1 . \mathrm{d} 0$, & 1.d0, & 1.d0, & $0 . \mathrm{d} 0$, & $0 . \mathrm{d} 0$, & $0 . \mathrm{d} 0 /$ \\
\hline DATA & Mii/1.d0, & $0 . \mathrm{d} 0$, & $0 . \mathrm{d} 0$, & $0 . \mathrm{d} 0$, & $0 . \mathrm{d} 0$, & $0 . \mathrm{d} 0$, \\
\hline & $0 . \mathrm{d} 0$ & 1.d0, & $0 . \mathrm{d} 0$, & $0 . \mathrm{d} 0$, & $0 . \mathrm{d} 0$, & $0 . \mathrm{d} 0$, \\
\hline & $0 . \mathrm{d} 0$, & $0 . \mathrm{d} 0$, & 1.d0, & $0 . \mathrm{d} 0$, & $0 . \mathrm{d} 0$, & $0 . \mathrm{d} 0$, \\
\hline & $0 . \mathrm{d} 0$, & $0 . \mathrm{d} 0$, & $0 . \mathrm{d} 0$, & 1.d0, & $0 . \mathrm{d} 0$, & $0 . \mathrm{dO}$, \\
\hline & & $0 . d 0$, & $0 . \mathrm{d} 0$, & $0 . \mathrm{d} 0$, & 1.d0, & $0 . \mathrm{d} 0$, \\
\hline & $0 . \mathrm{d} 0$, & $0 . \mathrm{d} 0$, & $0 . \mathrm{d} 0$, & $0 . \mathrm{d} 0$, & $0 . \mathrm{d} 0$, & \\
\hline
\end{tabular}

! Expected contents of Props (1..50)

1 : kappa slope of post yield compression line from e-ln p'-

diagram

! $2: \mathrm{ny}$

! 3 : lambda

! 4 : $\mathrm{M}$

Poisson's ratio

slope of swelling line from e-ln_p'-diagram

critical state $\mathrm{M}$ value (in triaxíal compression) 


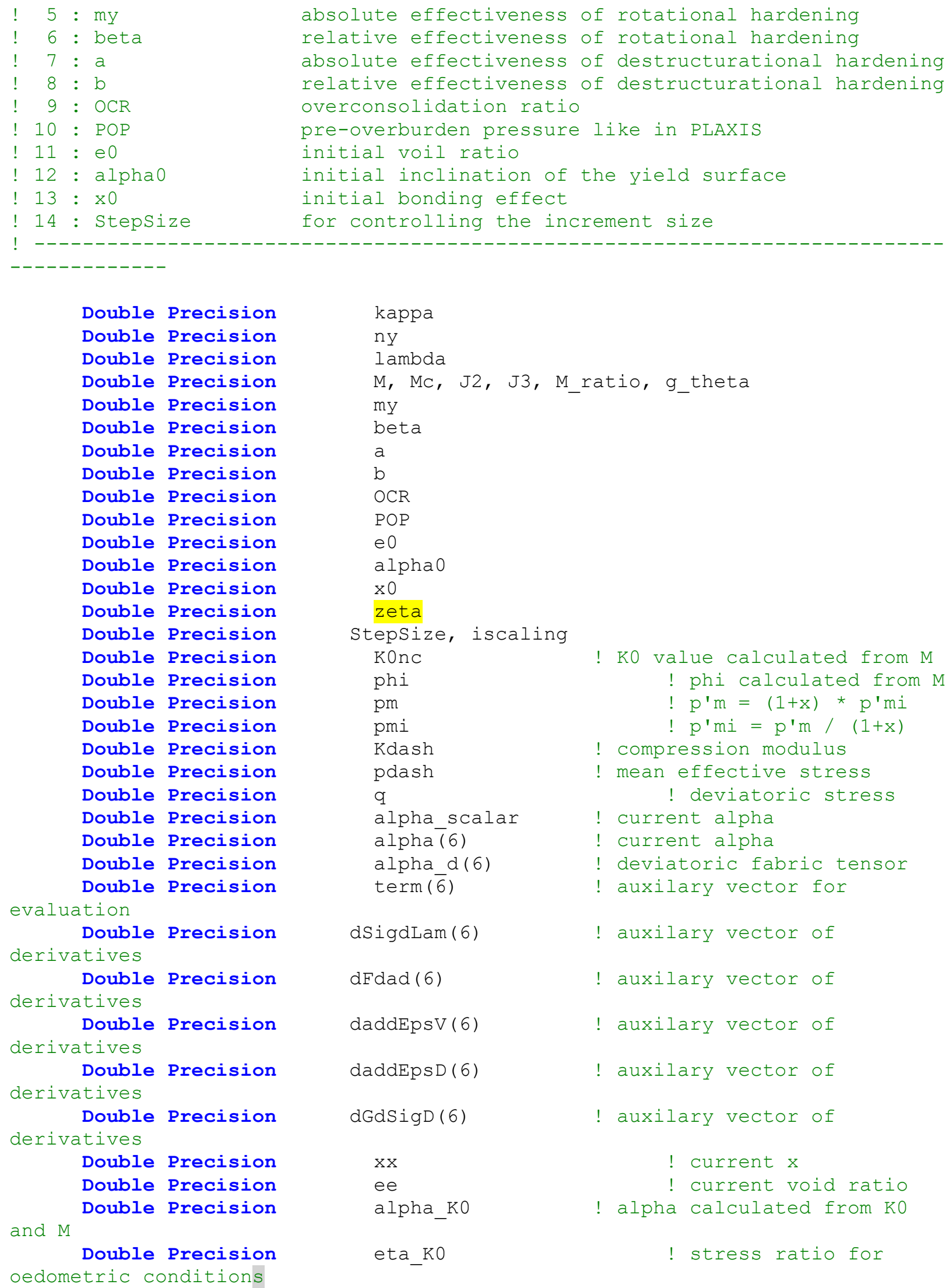




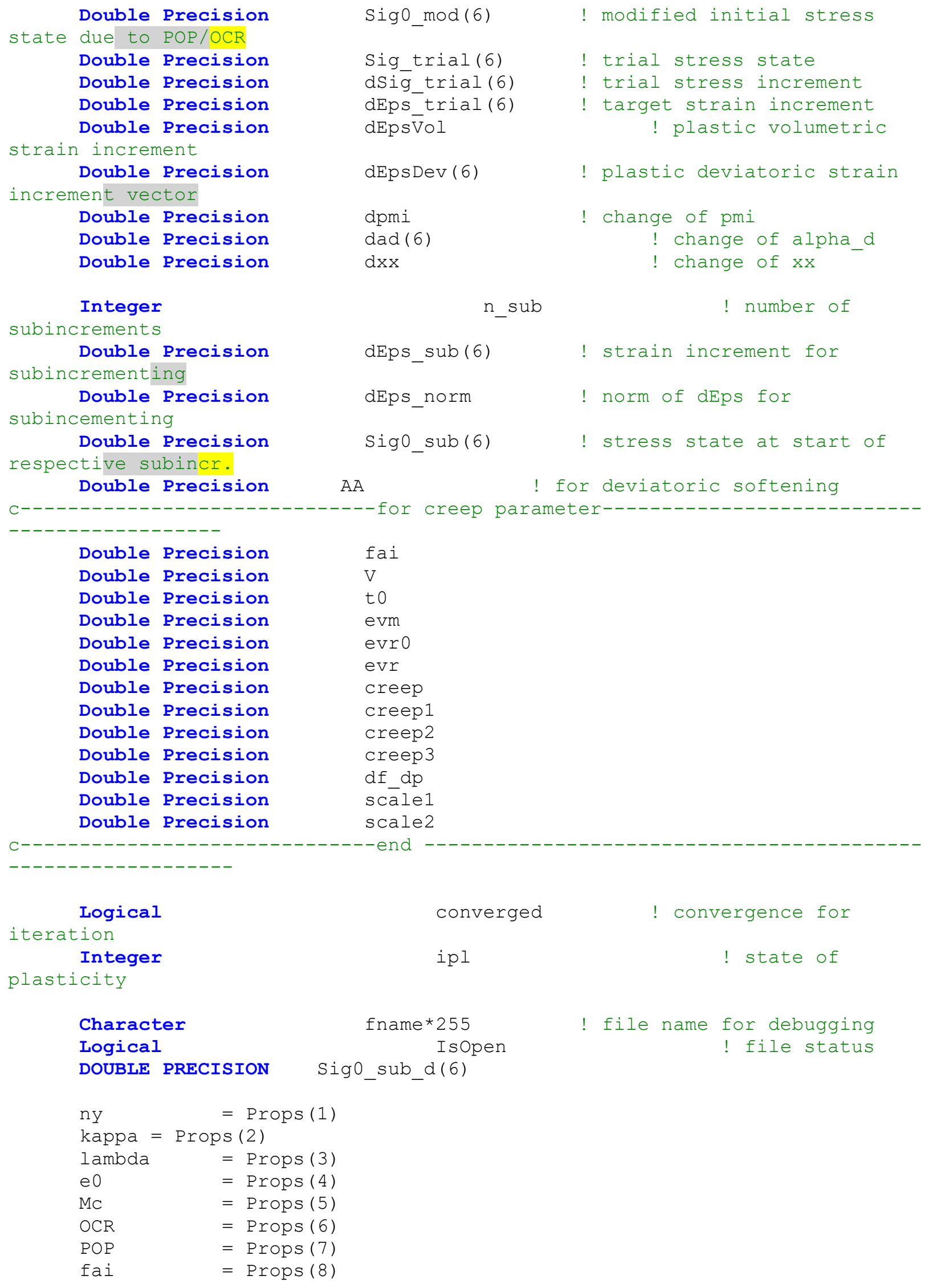


! calculate generalized alpha alpha_d $(1)=$ alpha $(1)-1$. alpha_d $(2)=$ alpha $(2)-1$. alpha_d $(3)=$ alpha $(3)-1$. alpha_d(4)=alpha $(4) * \operatorname{sqrt}(2$. alpha d (5) = alpha (5) * Sqrt (2.) alpha_d $(6)=$ alpha $(6) * \operatorname{sqrt}(2$.

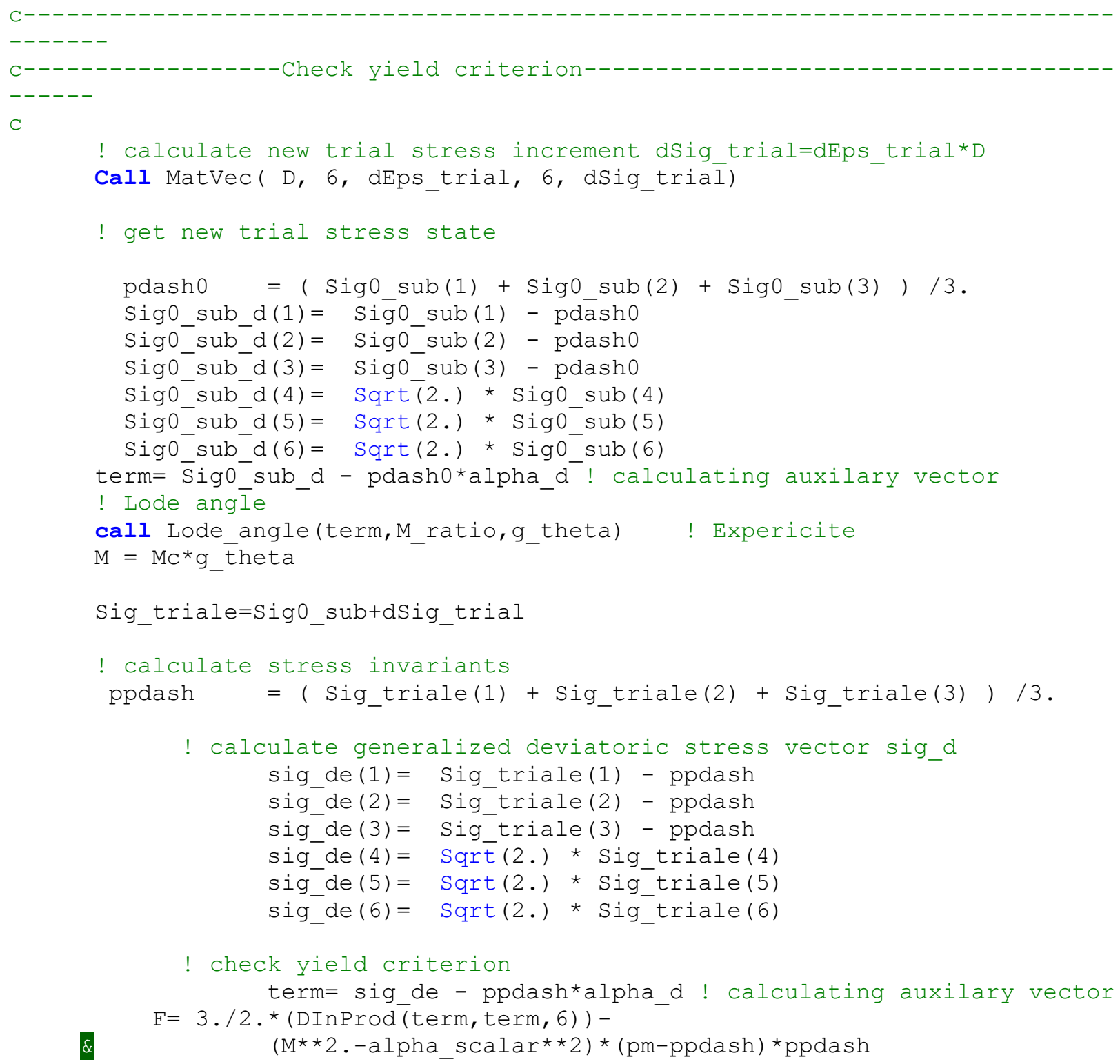


If (abs (ppdash) .ge. 5.) Then ! viscoplastic stress correction: associated flow

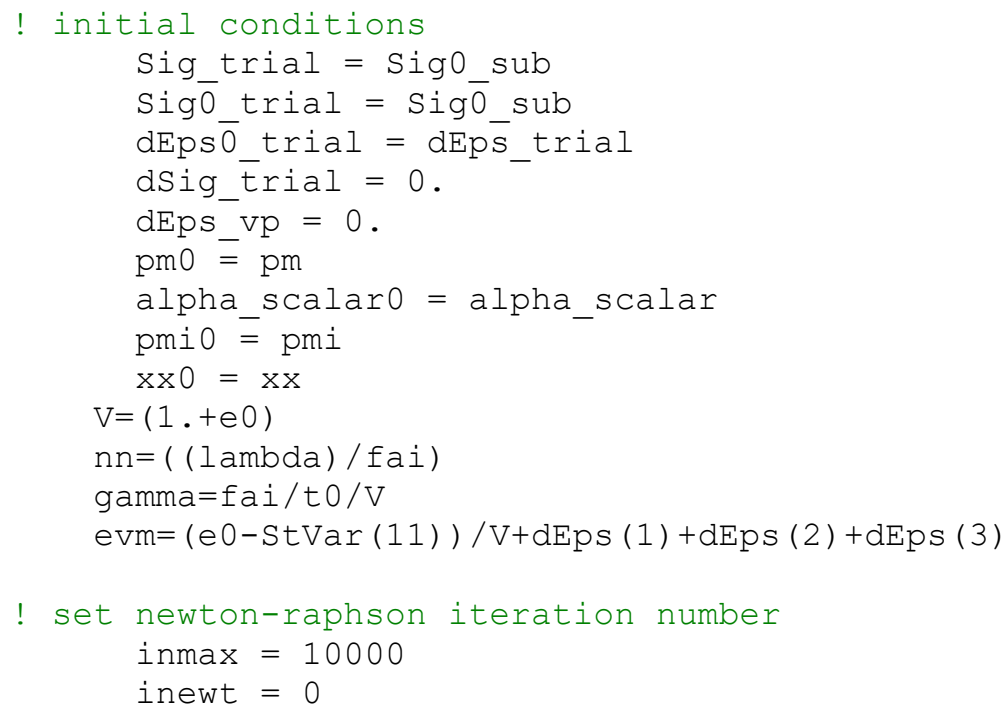




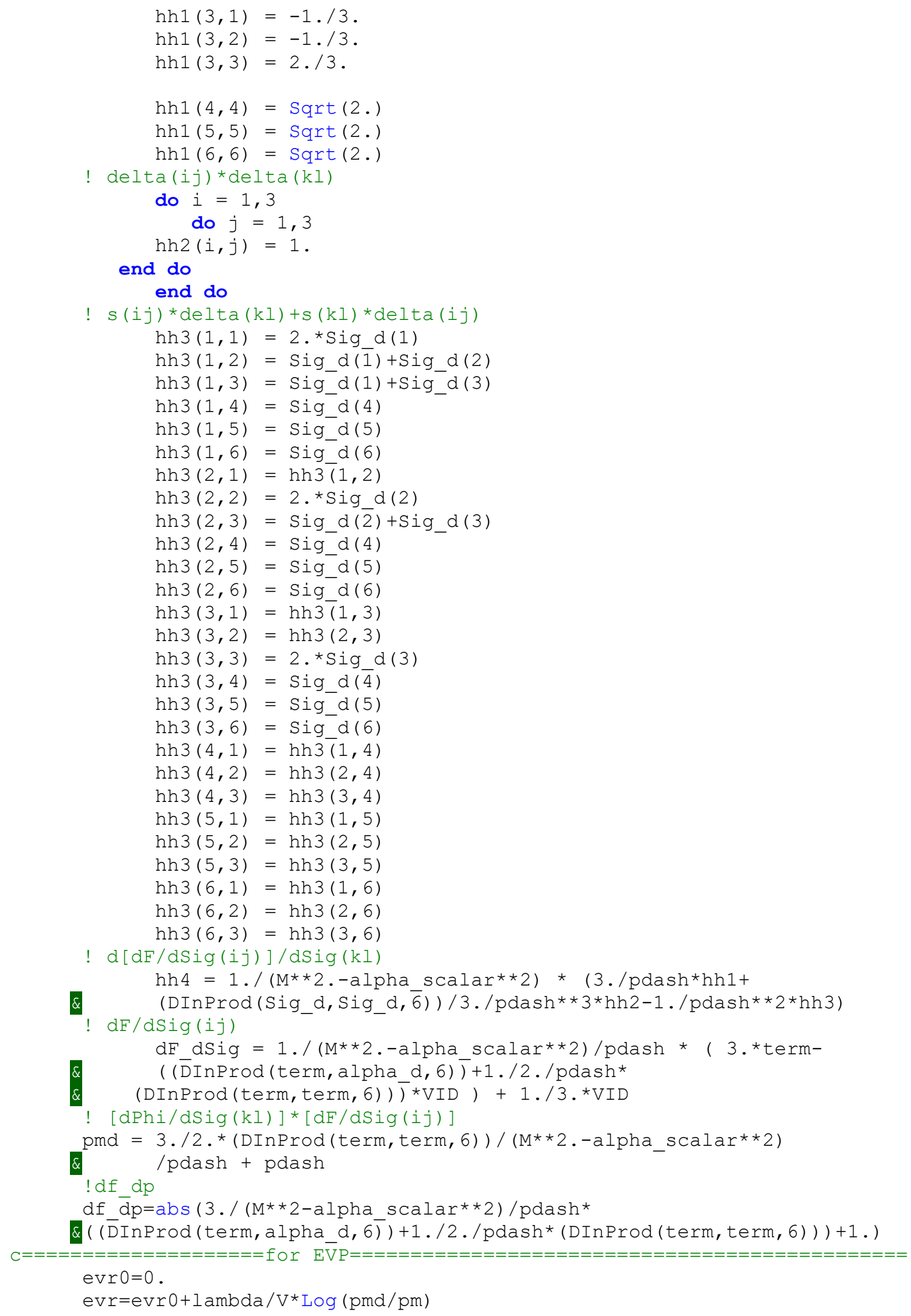




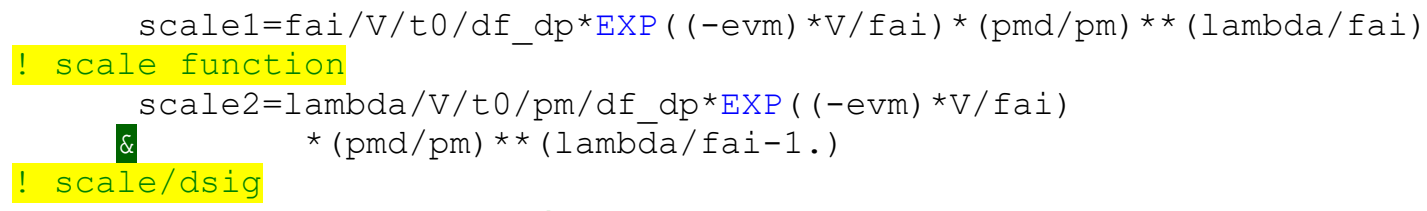

! $[\mathrm{H}]=$ dEps vpRate $(i j) / \mathrm{dSig}(\mathrm{kl})$ $\mathrm{hh}=(\mathrm{hh} \overline{5}+\mathrm{scale} 1 * \mathrm{hh} 4)$

! dGdSig dGdSig=hdd+dTime_sub*thet*hh call brinv (dGdSiḡ, 6, L)

!

$$
\mathrm{GG}=\mathrm{Q} Q-\mathrm{GG}
$$

call MatVec (dGdSig, 6,GG,6,dSig_trial) ! dSig

! update stress strain

Sig_trial=Sig_trial+dSig_trial

dōdSig_trial=Sig_trial-Sig0_trial

call MātVec (hdd, 6, dddSig_t̄iril, 6, ht3)

dEps_vp=dEps_trial-ht3

! calculate volumic plastic strain and deviatoric plastic strain

dEpsVol=dEps_vp (1) +dEps_vp (2) +dEps_vp (3)

dEps $\bar{V} \circ \mathrm{lMac}=\mathrm{dE \overline {p } s \mathrm { V }}$

if (dEpsVolMac.lt.0.) dEpsVolMac =0. !Macauley brackets

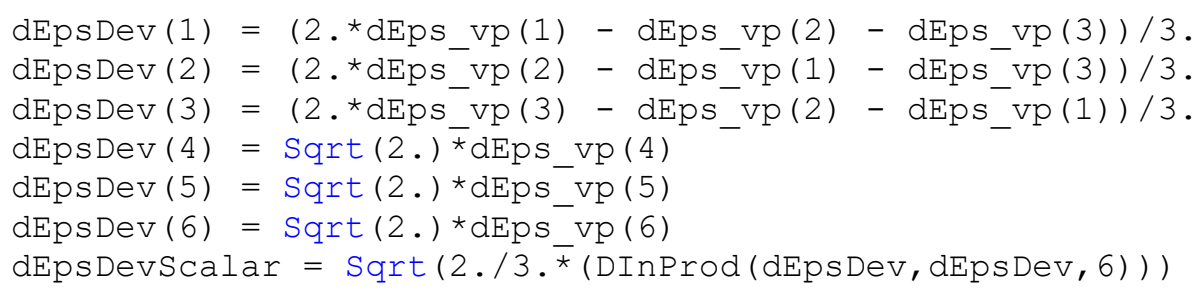




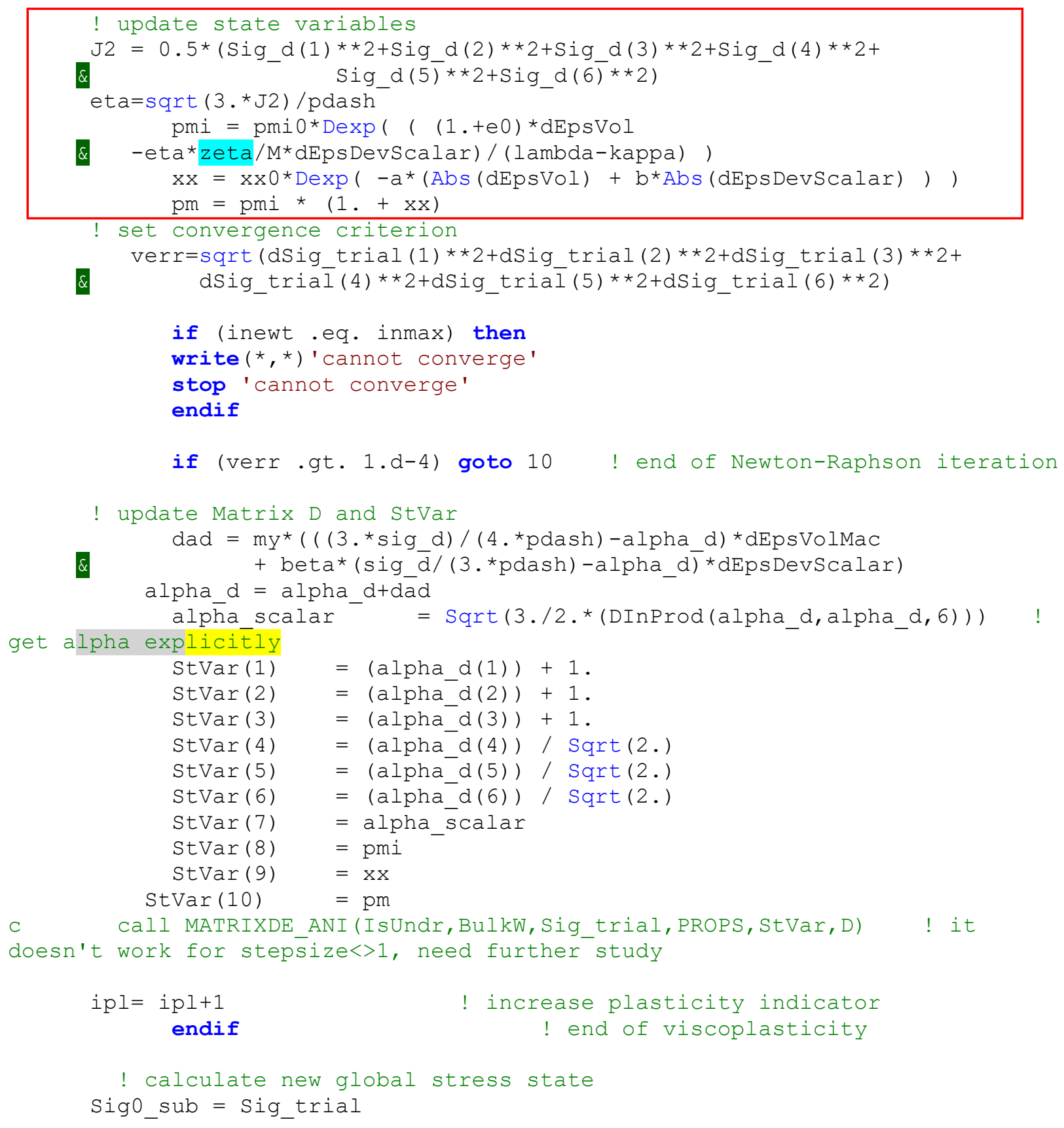

End Do ! Subincrementing

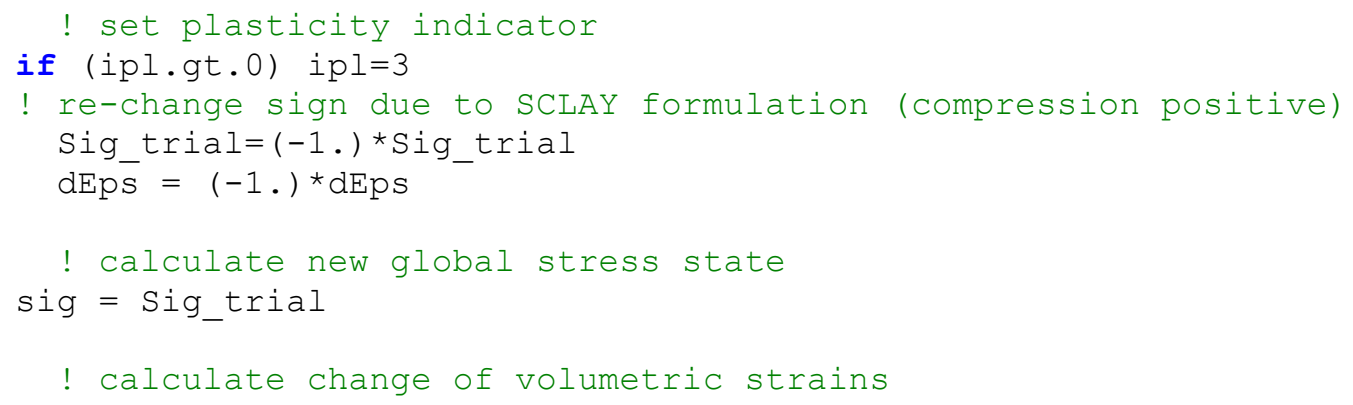


dEpsV $=$ dEps(1) + dEps(2) + dEps(3) ! stepsize donot work because ee donot updated during subincrementing

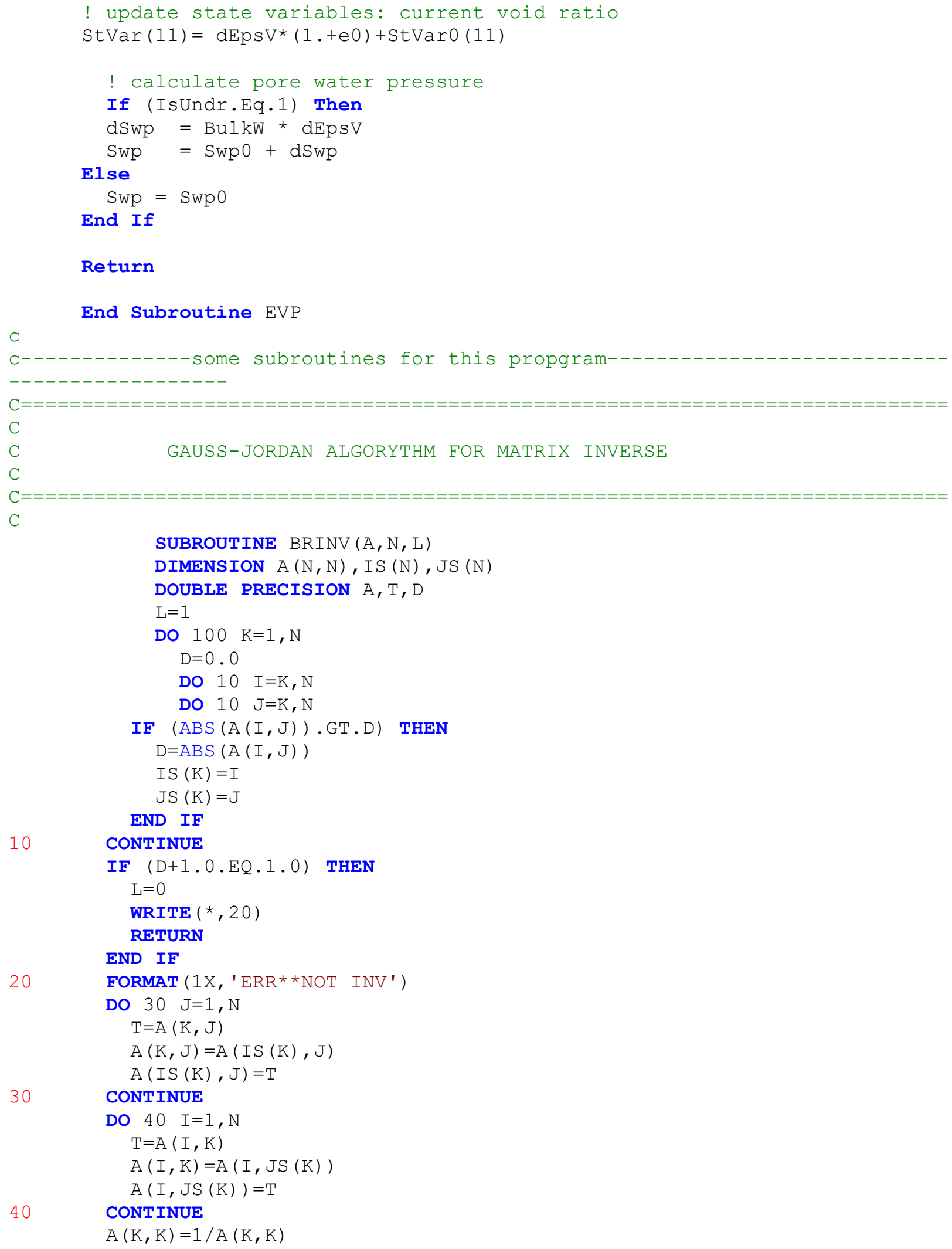




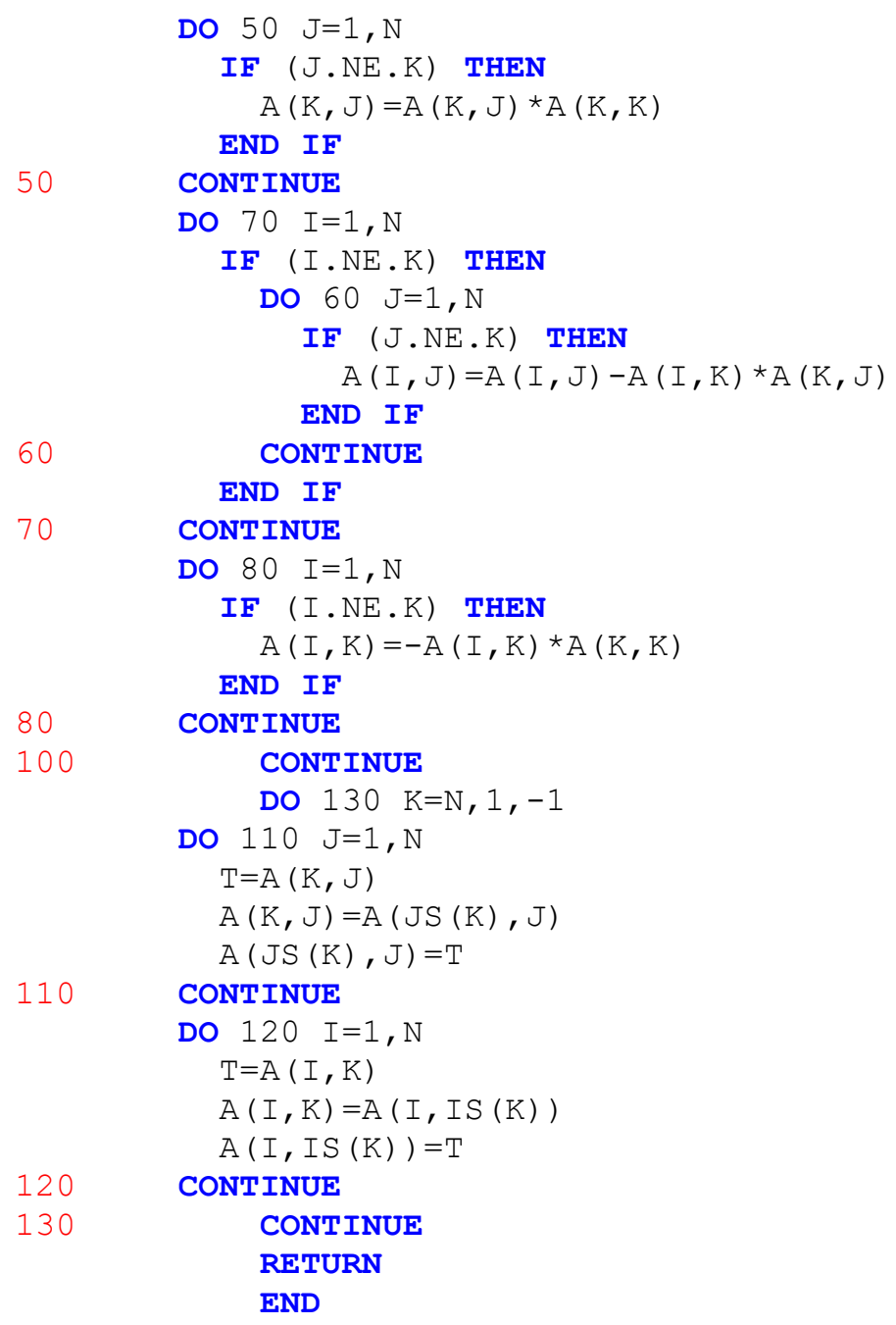




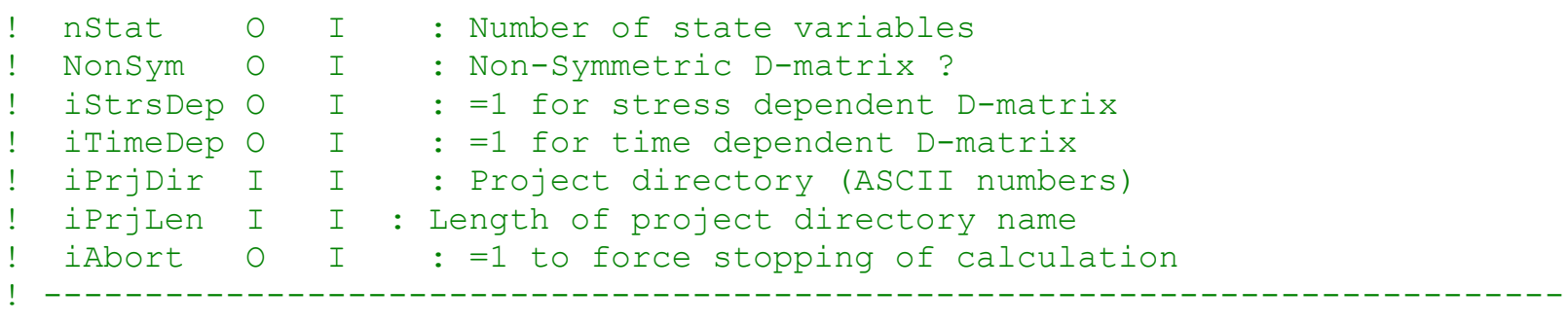

Implicit Double Precision (A-H, O-Z)

\begin{tabular}{ll} 
Integer & \multicolumn{1}{c}{ iPrjDir(iPrjLen) } \\
Double Precision & StVar(nStat) \\
Double Precision & Sig(6) \\
Double Precision & Sig0 (6) \\
Double Precision & StVar0 (nStat) \\
Double Precision & dEps (6) \\
Double Precision & D $(6,6)$ \\
Double Precision & Props $(50)$
\end{tabular}

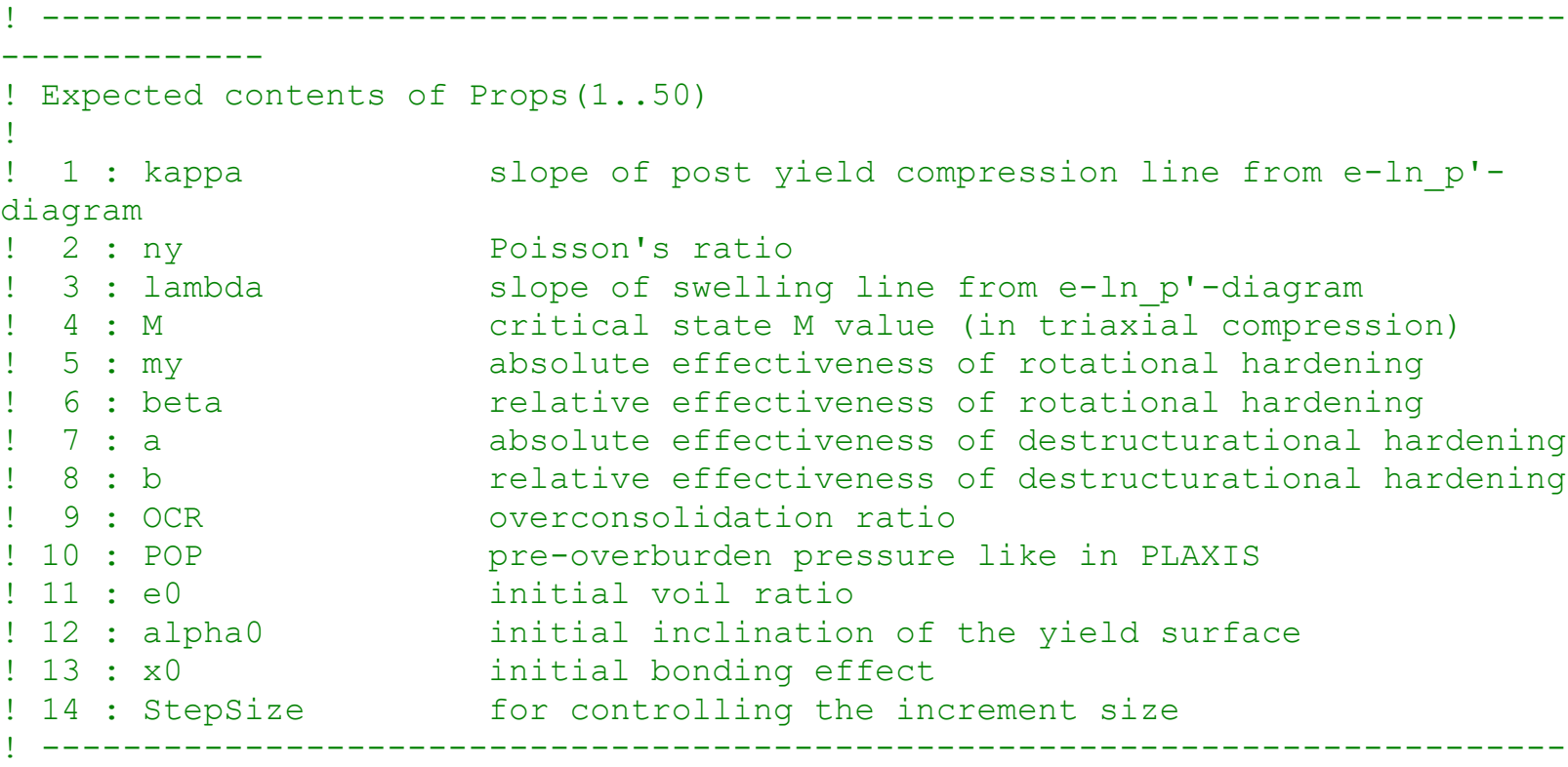

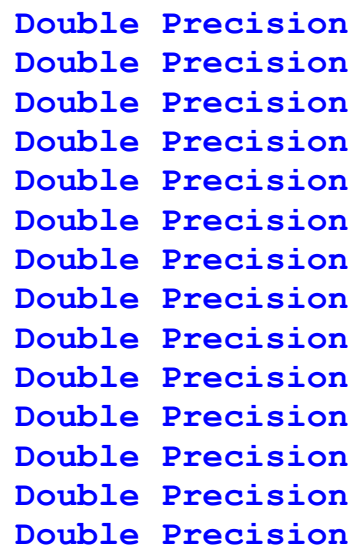

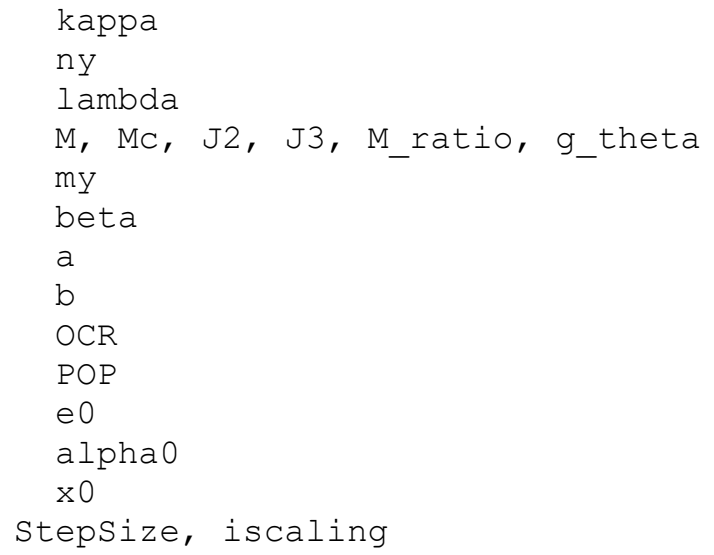




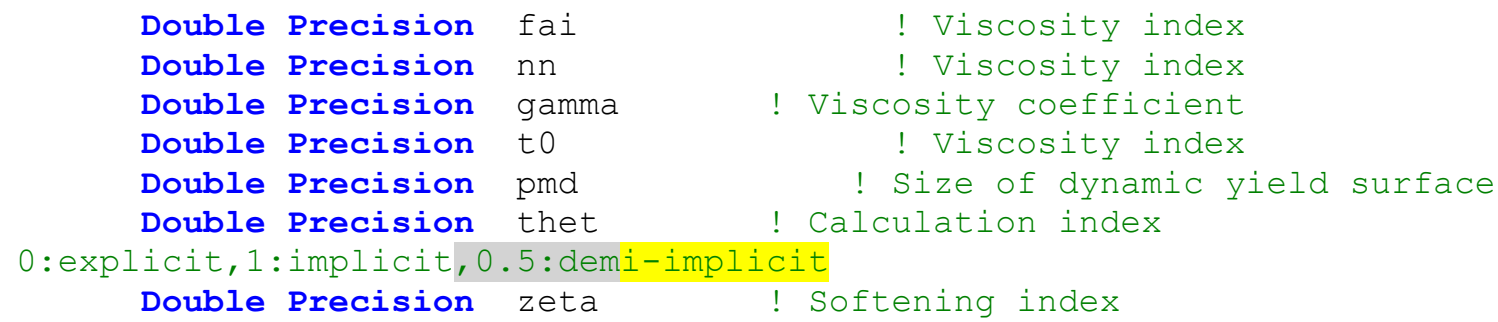

Double Precision Double Precision Double Precision Double Precision Double Precision Double Precision Double Precision Double Precision Double Precision Double Precision Double Precision Double Precision evaluation

Double Precision derivatives

Double Precision derivatives

Double Precision derivatives

Double Precision derivatives

Double Precision derivatives

Double Precision derivatives

Double Precision Double Precision Double Precision and $\mathrm{M}$

Double Precision oedometric conditions

Double Precision Double Precision state due to POP/OCR

Double Precision Double Precision

Double Precision Double Precision Double Precision

strain increment

Double Precision increment vector

Double Precision

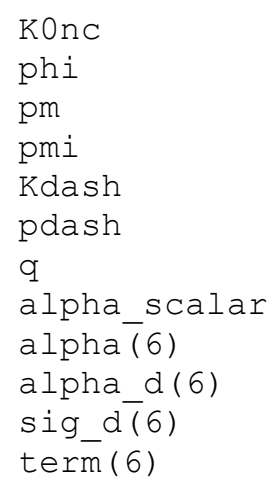

dEpsDev (6)

! plastic deviatoric strain

! K0 value calculated from M ! phi calculated from M ! $\mathrm{p}^{\prime} \mathrm{m}=(1+\mathrm{x}) * \mathrm{p}^{\prime} \mathrm{mi}$

! $\mathrm{p}^{\prime} \mathrm{mi}=\mathrm{p}^{\prime} \mathrm{m} /(1+\mathrm{x})$

! compression modulus

! mean effective stress

! deviatoric stress

! current alpha

! current alpha

! deviatoric fabric tensor

! deviatoric stress vector

! auxilary vector for

! auxilary vector of

! auxilary vector of

! auxilary vector of

! auxilary vector of

! auxilary vector of

! auxilary vector of

! current $\mathrm{x}$

! current void ratio

! alpha calculated from KO

! stress ratio for

! stress increment

! modified initial stress

! trial stress state

! trial stress increment

! target strain increment

! plastic strain increment ! plastic volumetric

dpmi
! change of pmi 
Double Precision

Double Precision

Integer

subincrements

Double Precision

subincrementing

Double Precision

subincementing

Double Precision

respective subincr.

Logical

iteration

Integer

plasticity

Character

debugging

Logical

DOUBLE PRECISION

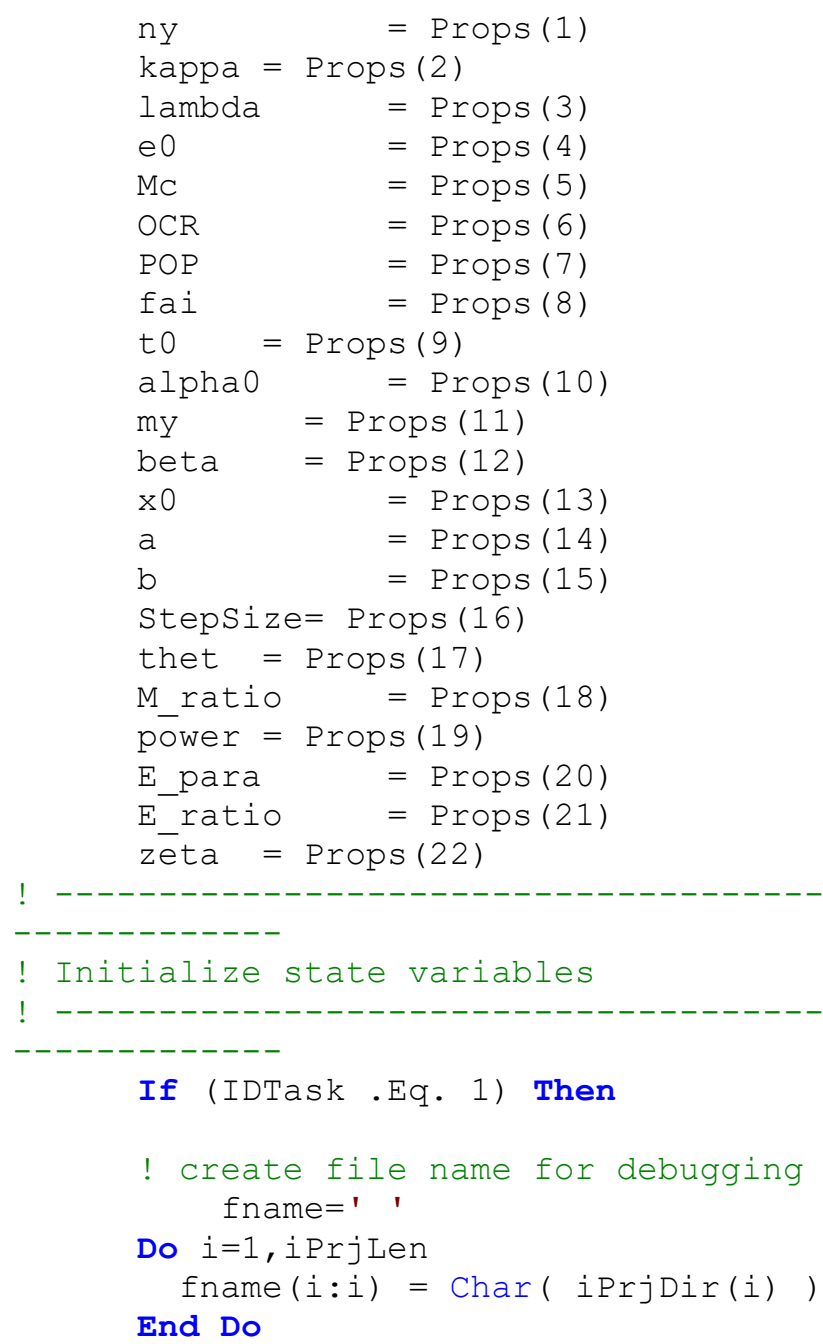


fname $=$ fname $(:$ iPrjLen) $/ /$ ' \usrdbg.zyin'

! open debugging file

Inquire (Unit $=1$, Opened= IsOpen)

If (.not.Isopen) Then

Open (Unit=1, File= fname, Position= 'append')

Write $\left(1,{ }^{*}\right)$ 'starting next phase'

\section{End If}

! do IDTaskl only once

If (StVar0 (12)==123.) Return

! create debugging file

If ( $i E l==1$. and. $i n t e==1$ ) Then

Close (Unit $=1$, Status $=$ 'delete')

Open (Unit= 1, File $=$ fname)

Write $(1, *)$ 'initialization'

Call Wrivec (1,' Props...' ', Props, 21)

End If

! checking input variables

if ( (OCR.ne.0.) and. (POP.ne.0.)) then ! using POP and OCR together is not possible

Write $(1, *)$ 'ERROR: using POP and OCR together is not possible'

Stop

end if

if (POP.gt.0.) then ! POP has to be negative (compression=negative)

Write $(1, *)$ 'ERROR: POP has to be negative (compression=negative)'

Stop

end if

if (OCR.lt.o.) then ! negative OCR values are not possible

Write $\left(1,{ }^{*}\right)$ 'ERROR: negative OCR values are not possible'

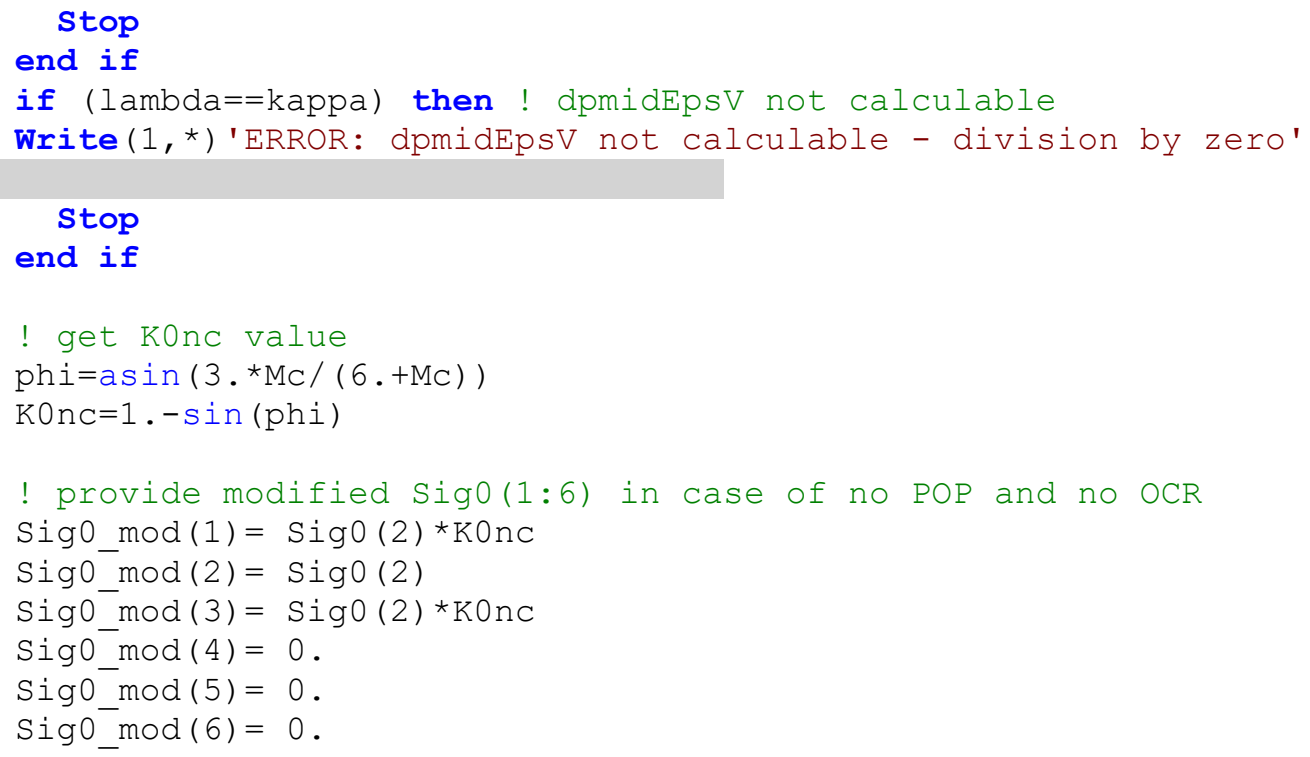




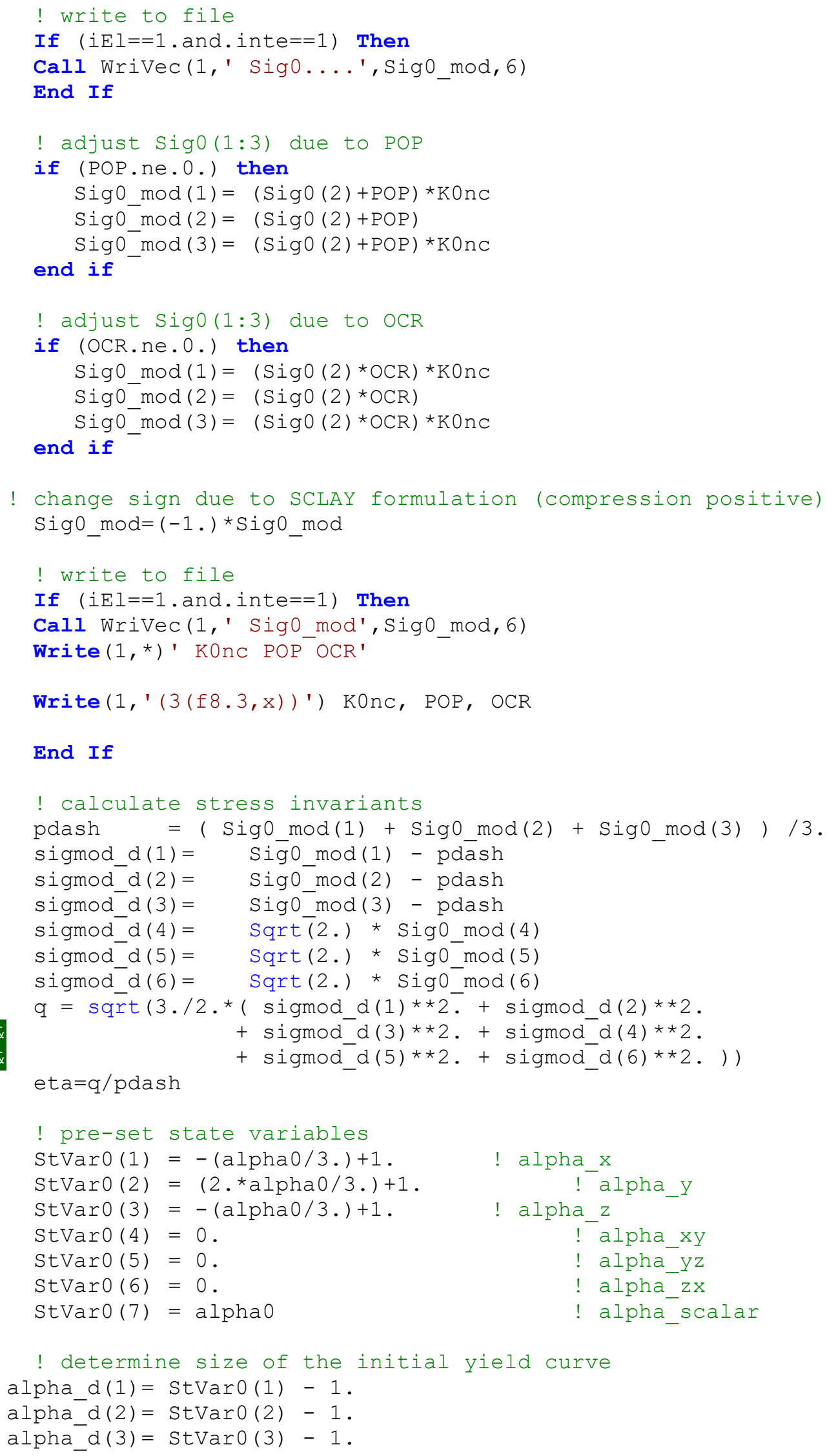




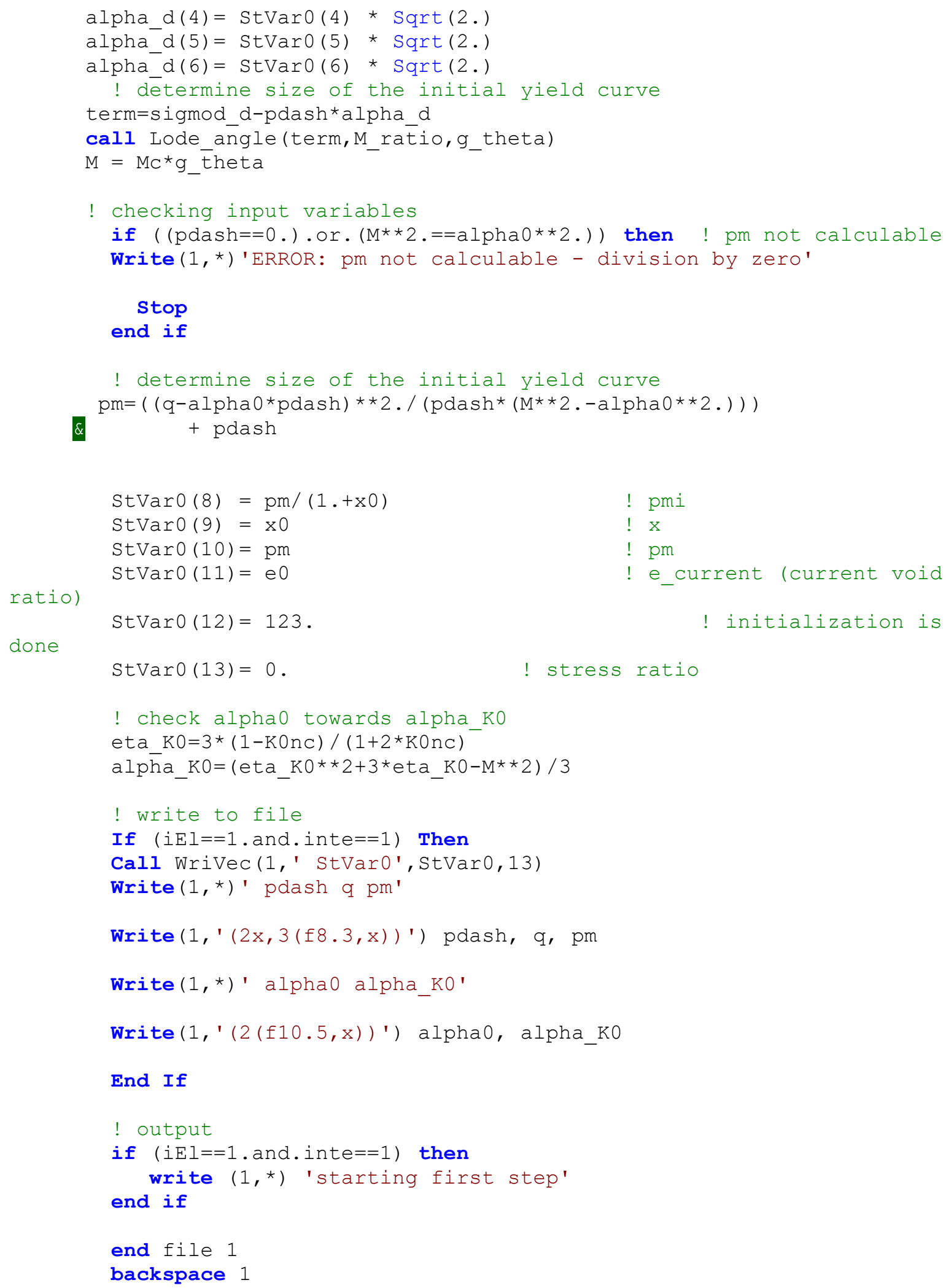

End If $!$ IDTask $=1$ 


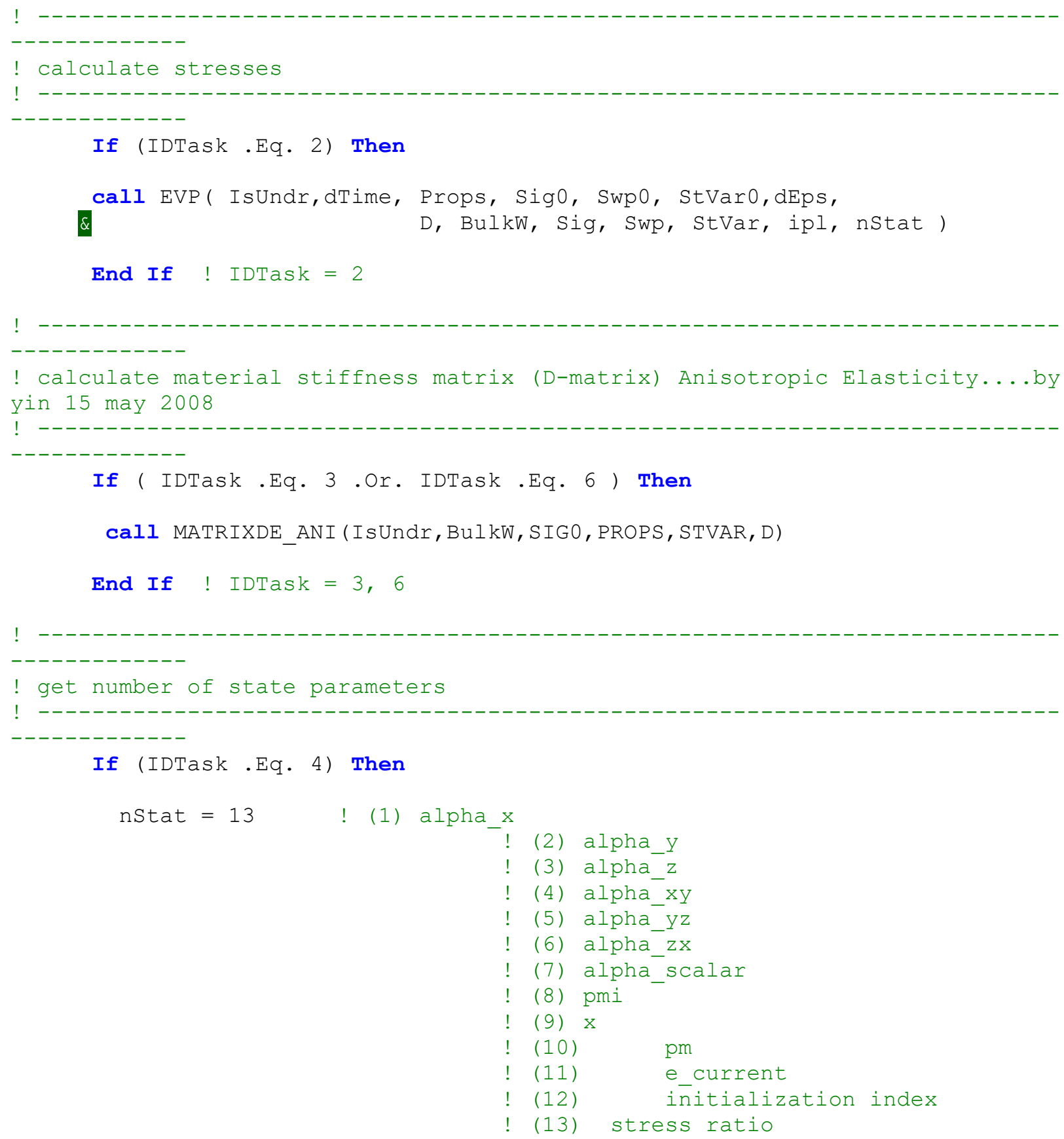

End If ! IDTask $=4$

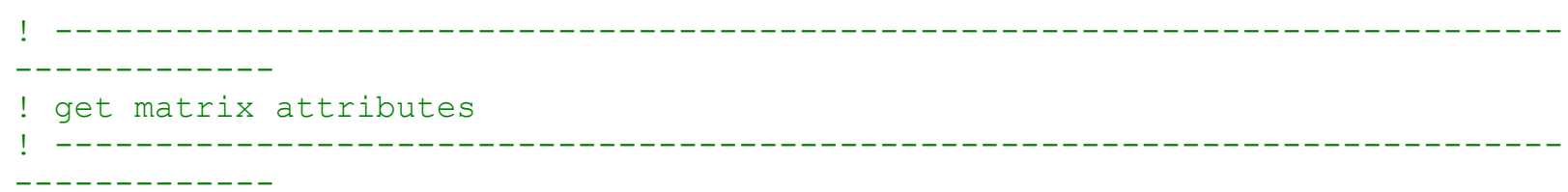

If (IDTask .Eq. 5) Then

NonSym = 0 ! 1 for non-symmetric D-matrix

istrsDep $=1$ ! 1 for stress dependent D-matrix

itang $=0$ ! 1 for tangent D-matrix 
iTimeDep $=0$ ! 1 for time dependent D-matrix

End If ! IDTask $=5$

Return

End Subroutine MY_SCLAY

$\mathrm{C}$

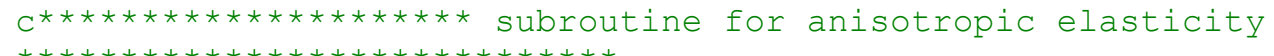

SUBROUTINE MATRIXDE_ANI (IsUndr,BulkW, SIG, PROPS, STVAR, D)

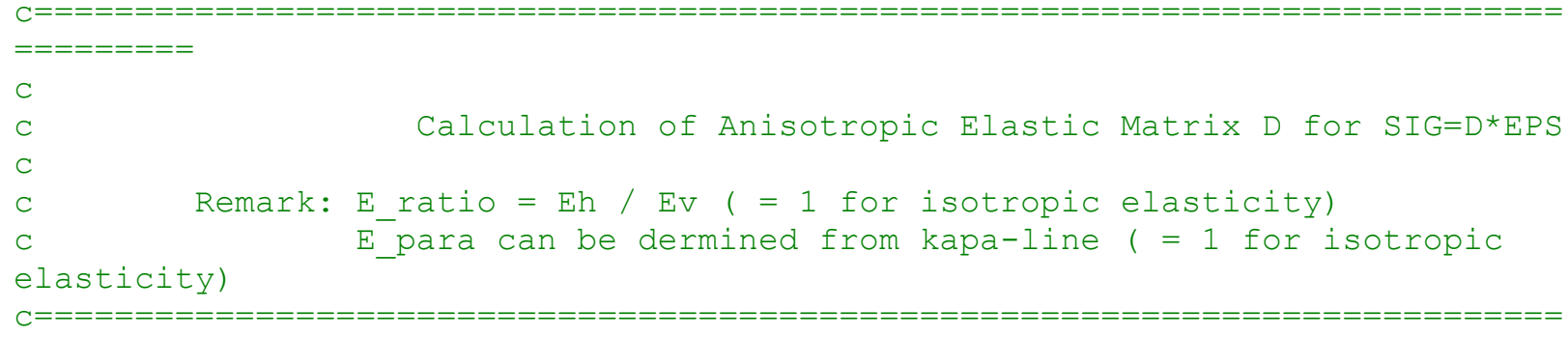




$$
\begin{aligned}
& \mathrm{D}(2,3)=\mathrm{F} 1 * x \mathrm{Nu}^{*} \text { alph } \\
& \mathrm{D}(2,1)=\mathrm{D}(1,2) \\
& \mathrm{D}(3,1)=\mathrm{D}(1,3) \\
& \mathrm{D}(3,2)=\mathrm{D}(2,3)
\end{aligned}
$$

\section{tensor}

$\mathrm{G}=0.5{ }^{\star} \mathrm{E} /(1 .+\mathrm{xNu}) * \mathrm{al} \mathrm{ph}$

!calculate bulk modulus

$\mathrm{BulkW}=0$

If (IsUndr.Eq.1) Then

$\mathrm{xNu} \mathrm{U}=0.495 \mathrm{~d} 0$

$\mathrm{FaC}=\left(1+\mathrm{xNu} \mathrm{U}_{\mathrm{U}}\right) /\left(1-2 * \mathrm{xNu} \mathrm{U}_{\mathrm{U}}\right)-(1+\mathrm{xNu}) /(1-2 * \mathrm{xNu})$

$\mathrm{FaC}=2 \mathrm{D} 0 *_{\mathrm{G}} / \overline{3} \mathrm{D} 0 * \mathrm{FaC}$

$\mathrm{BulkW}=\mathrm{FaC}$

End If

END 


\section{REFERENCES}

Aboshi, H., Yoshikumi, H., and Maruyama, S. 1970. Constant loading rate consolidation test. Soils and Foundations, $\mathbf{X}(1)$ : 43-56.

Acres Consulting Services, L. 1977. Construction Aspects and Initial Post-Construction Performance.

Ahmad, A. 2018. Leaching impact on compressibility of untreated and cement-treated Champlain Sea clay. Ryerson University.

Ahmadi, H., Rahimi, H., and Soroush, A. 2011. Investigation on the Characteristics of Pore Water Flow During CRS Consolidation Test. Geotech Geol Eng,. doi:10.1007/s10706-011-9431-z.

Ahmadi, H., Rahimi, H., Soroush, A., and Alen, C. 2014. Experimental research on variation of pore water pressure in constant rate of strain consolidation test. Acta Geotechnica Slovenica, 47-57.

ASTM. 2015. D4186/D4186M-12: Standard Test Method for One-Dimensional Consolidation Properties of Saturated Cohesive Soils Using Controlled-Strain Loading. doi:10.1520/D4186.

Banimahd, M., and Woodward, P.K. 2006. Load-displacement and bearing capacity of foundations on granular soils using a multi-surface kinematic constitutive soil model. International Journal for Numerical and Analytical Methods in Geomechanics, 30(9): 865886. doi:10.1002/nag.502.

Barden, L. 1969. Time-dependent deforamtion of normally consolidated clays and peats. Journal of the Soil Mechanics and Foundation Division, ASCE, 95(1): 1-32.

Baudet, B., and Stallebrass, S. 2004. A constitutive model for structured clays. Géotechnique, 54(4): 269-278. doi:10.1680/geot.2004.54.4.269.

Bjerrum, L. 1967. Engineering Geology of Norwegian Normally-Consolidated Marine Clays as Related to Settlements of Buildings. Géotechnique, 17(2): 83-118. doi:10.1680/geot.1967.17.2.83.

Borja, R.I., and Kavazanjian, E. 1985. A constitutive model for the stress-strain-time behaviour of "wet” clays. Géotechnique, 35(3): 283-298. doi:10.1680/geot.2004.54.4.269.

Bozozuk, M. 1973. Settlement of an Embankment Constructed on Marine Clay: Discussion. Canadian Geotechnical Journal, 10(4): 670. doi:10.1139/t73-064.

Burland, J.B. 1990. On the compressibility and shear strength of natural clays. Géotechnique, 40(3): 329-378.

Burn, K.N. 1969. Settlement of a High Embankment and Overpass Structures in Ottawa. 6(33): $33-45$.

Burn, K.N., and Hamiltion, J.J. 1968. Settlement of an embankment on Leda Clay. Canadian Geotechnical Journal, 5(1).

Callisto, L., and Calabresi, G. 1998. Mechanical behaviour of a natural soft clay. Géotechnique, 48(4): 495-513.

Callisto, L., and Rampello, S. 2004. An interpretation of structural degradation for three natural 
clays. Canadian Geotechnical Journal, 41(3): 392-407. doi:10.1139/T03-099.

CGS. 2006. Canadian Foundation Engineering Manual, 4th Edition.

Chai, J., and Miura, N. 1999. Investigation of factors affecting vertical drain behavior. Journal of Geotechnical and Geoenvironmental Engineering, 125(March): 216-226.

Chai, J.C., Shen, J.S.L., Liu, M.D., and Yuan, D.J. 2018. Predicting the performance of embankments on PVD-improved subsoils. Computers and Geotechnics, 93: 222-231. Elsevier Ltd. doi:10.1016/j.compgeo.2017.05.018.

Chang, C.S., and Hicher, P.Y. 2005. An elasto-plastic model for granular materials with microstructural consideration. International Journal of Solids and Structures, 42(14): 42584277. doi:10.1016/j.ijsolstr.2004.09.021.

Chen, S.L., and Abousleiman, Y.N. 2018. Cavity expansion in strain hardening frictional soils under drained condition. International Journal for Numerical and Analytical Methods in Geomechanics, 42(1): 132-142. doi:10.1002/nag.2718.

Cloutier, V., Lefebvre, R., Savard, M.M., and Therrien, R. 2009. Desalination of a sedimentary rock aquifer system invaded by Pleistocene Champlain Sea water and processes controlling groundwater geochemistry. Environmental Earth Sciences, 59(5): 977-994. doi:10.1007/s12665-009-0091-8.

Collins, I.F., and Kelly, P.A. 2002. A thermomechanical analysis of a family of soil models. Géotechnique, 52(7): 507-518. doi:10.1680/geot.2002.52.7.507.

Conte, E., Donato, A., and Troncone, A. 2013. Progressive failure analysis of shallow foundations on soils with strain-softening behaviour. Computers and Geotechnics, 54: 117-124. Elsevier Ltd. doi:10.1016/j.compgeo.2013.07.002.

Cotecchia, F., and Chandler, R.J. 2000. A general framework for the mechanical behaviour of clays. Géotechnique, 50(4): 431-447. doi:10.1680/geot.2000.50.4.431.

Crawford, C., and Bozozuk, M. 1990. Thirty years of secondary consolidation in sensitive marine clay. Canadian Geotechnical Journal, 27: 315-319. doi:10.1139/t91-060.

Crawford, C.B. 1953. Settlement studies on the National Museum Building, Ottawa, Canada.

Cucovillo, T., and Coop, M.R. 1999. On the mechanics of structured sands. Géotechnique, 49(6): 741-760. doi:doi:10.1680/geot.1999.49.6.741.

Cudny, M. 2011. Numerical analysis of test embankment on soft ground using multi-laminate type model with destructuration. Archives of Civil Engineering, 57(1): 27-44. doi:10.2478/v.10169-011-0004-6.

Cudny, M., and Vermeer, P.A. 2004. On the modelling of anisotropy and destructuration of soft clays within the multi-laminate framework. Computers and Geotechnics, 31(1): 1-22. doi:10.1016/j.compgeo.2003.12.001.

Dafalias, Y. 1986. An anisotropic critical state soil plasticity model. Mechanics Research Communications, 13(6): 341-347. doi:10.1080/08977190802066655.

Dafalias, Y., Majid, T.M., and Papadimitrious, A.G. 2006. Saniclay: simple anisotropuc clay plasticity model. International Journal for Numerical and Analytical Methods in 
Geomechanics, 30(1): 1231-1257. doi:10.1002/nag.

Dafalias, Y., Manzari, M.T., and Akaishi, M. 2002. A simple anisotropic clay plasticity model. Mechanics Research Communications, 29(4): 241-245. doi:10.1016/S0093-6413(02)002525.

Desaulniers, D.E., and Cherry, J.A. 1989. Origin and movement of groundwater and major ions in a thick deposit of Champlain Sea clay near Montréal. Canadian Geotechnical Journal, 26(1): 80-89. doi:10.1139/t89-009.

Eden, W.J., and Mitchell, R.J. 1970. The mechanics of landslides in Leda clay. Canadian Geotechnical Journal, 7: 285296.

Einav, I., and Randolph, M.F. 2005. Combining upper bound and strain path methods for evaluating penetration resistance. International Journal for Numerical Methods in Engineering, 63(14): 1991-2016. doi:10.1002/nme.1350.

Fatahi, B., Le, T.M., Le, M.Q., and Khabbaz, H. 2013. Soil creep effects on ground lateral deformation and pore water pressure under embankments. Geomechanics and Geoengineering, 8(2): 107-124. doi:10.1080/17486025.2012.727037.

Feng, W., Yin, J., and Yin, Z. 2014. Elasto-ViscoPlastic model ( EVP-Yin ) as User Defined Soil Model for PLAXIS User Manual. (October).

Garlanger, J.E. 1972. The consolidation of soils exhibiting creep under constant effective stress. Géotechnique, 22(1): 71-78. doi:doi:10.1680/geot.1972.22.1.71.

Gasparre, A., Nishimura, S., Coop, M.R., and Jardine, R.J. 2007. The influence of structure on the behaviour of London Clay. Géotechnique, 5(1): 19-31. doi:geot.2007.57.1.19.

Gillott, J.E. 1970. Fabric of Leda clay investigated by optical, electron-optical, and X-ray diffraction methods. Engineering Geology, 4(2): 133-153. doi:10.1016/00137952(70)90009-8.

Graham, J., Crooks, J.H.A., and Bell, A.L. 1983. Time effects on the stress-strain behaviour of natural soft clays. Géotechnique, 33(3): 327-340. doi:doi:10.1680/geot.1983.33.3.327.

Graham, J., Noonan, M.L., and Lew, K. V. 1984. Yield states and stress-strain relationships in a natural plastic clay: Reply. Canadian Geotechnical Journal, 21(3): 598-599. doi:10.1139/t84064.

Hamouche, K.K., Leroueil, S., Roy, M., and Lutenegger, A.J. 1995. In situ evaluation of Ko in eastern Canada clays. Canadian Geotechnical Journal, 32(Marchetti 1980): 677-688.

Hinchberger, S.D., Qu, G., and Lo, K.Y. 2010. Constitutive approach for rate-sensitive anisotropic structured clays. International Journal for Numerical and Analytical Methods in Geomechanics, 34(17): 609-626. doi:10.1002/nag.881.

Holtz, R.D., Kovacs, W.D., and T.C., S. 2010. An Introduction to Geotechnical Engineering (Second Edition). Upper Saddle River: Pearson Education, Inc.

Hong, Z., Shen, S., Deng, Y., and Negami, T. 2007. Loss of soil structure for natural sedimentary clays. In In Proceedings of the Institution of Civil Engineers - Geotechnical Engineering. pp. 153-159. doi:doi:10.1680/geng.2007.160.3.153. 
Indraratna, B., Balasubramaniam, A.S., and Sivaneswaran, N. 1997. Analysis of settlement and lateral deformation of soft clay foundation beneath two full-scale embankments. International Journal for Numerical and Analytical Methods in Geomechanics, 21: 599-618. doi:10.3208/sandf1972.32.117.

Indraratna, B.B., Balasubramaniam, A.S., and Ratnayake, P. 1994. Performance of embankment stabilized with vertical drains on soft clay. Journal of Geotechnical Engineering, 120(2): 257-273.

José, M., and Jacinto, A. 2018. What is clay? A new definition of "clay" based on plasticity and its impact on the most widespread soil classification systems. Applied Clay Science, 161(November 2017): 57-63. Elsevier. doi:10.1016/j.clay.2018.04.011.

Karim, M.R., Oka, F., Krabbenhoft, K., Leroueil, S., and Kimoto, S. 2013. Simulation of LongTerm Consolidation Behavior of Soft Sensitive Clay using an Elasto-Viscoplastic Constitutive Model. International Journal for Numerical and Analytical Methods in Geomechanics, 37(1): 2801-2824. doi:10.1002/nag.

Karstunen, M., and Koskinen, M. 2008. Plastic Anisotropy of Soft Reconstituted Clays. Canadian Geotechnical Journal, 45(3): 314-328. doi:10.1139/T07-073.

Karstunen, M., and Yin, Z.Y. 2010. Modelling time-dependent behaviour of Murro test embankment. Geotechnique, 60(10): 735-749. doi:10.1680/geot.8.P.027.

Kelly, R.B., Sloan, S.W., Pineda, J.A., Kouretzis, G., and Huang, J. 2018. Outcomes of the Newcastle symposium for the prediction of embankment behaviour on soft soil. Computers and Geotechnics, 93: 9-41. Elsevier Ltd. doi:10.1016/j.compgeo.2017.08.005.

Kempfert, H., and Berhane, G. 2006. Excavations and foundations in soft soils. Springer Science \& Business Media.

Kirstein, A. 2017. Evaluation of different methods to identify preconsolidation pressure of Champlain Sea clay from CRS tests. Ryerson University.

Koskinen, M. 2014. Plastic anisotropy and destructuration of soft Finnish clays. Aalto University.

Koskinen, M., Karstunen, M., and Wheeler, S.J. 2002. Modelling destructuration and anisotropy of a natural soft clay. In In Proceedings of the 5th European Conference on Numerical Methods in Geotechnical Engineering, Paris, France. pp. 11-20.

Krenk, S. 2000. Characteristic state plasticity for granular materials Part I: Basic theory. International Journal of Solids and Structures, 37(43): 6343-6360. doi:10.1016/S00207683(99)00278-4.

Krenn, H. 2008. Numerical modelling of embankments on soft soils. University of Strathclyde. Available from http://ethos.bl.uk/OrderDetails.do?uin=uk.bl.ethos.237991.

Kutter, B.L., and Sathialingam, N. 1992. Elastic-viscoplastic modelling of the rate-dependent behaviour of clays. Géotechnique, 42(3): 427-441. doi:doi:10.1680/geot.1992.42.3.427.

Lade, P. V. 1977. Elasto-plastic stress-strain theory for cohesionless soil with curved yield surfaces. International Journal of Solids and Structures, 13(11): 1019-1035. Pergamon Press Ltd. doi:10.1016/0020-7683(77)90073-7.

Lade, P. V. 1976. Stress-path dependent behaviour of cohesionless soil. Journal of the 
Geotechnical Engineering Division, ASCE, 102(GT1): 51-68.

Larsson, R., and Sallfors, G. 1985. Automatic continuous consolidation testing in Sweden. In In Consolidation of soils: testing and evaluation, Proceedings of the ASTM Committee D-18 Symposium on Soil and Rock, Orlando. pp. 299-328.

Lashkari, A. 2010. A SANISAND model with anisotropic elasticity. Soil Dynamics and Earthquake Engineering, 30(12): 1462-1477. Elsevier. doi:10.1016/j.soildyn.2010.06.015.

Lefebvre, G., Bozozuk, M., Philibert, a., and Hornych, P. 1991. Evaluating K o in Champlain clays with hydraulic fracture tests. Canadian Geotechnical Journal, 28(3): 365-377. doi:10.1139/t91-047.

Leoni, M., Karstunen, M., and Vermeer, P.A. 2008. Anisotropic creep model for soft soils. Géotechnique, 58(3): 215-226. doi:10.1680/geot.2008.58.3.215.

Leroueil, S. 1996. Compressibility of clays: fundamental and practical aspects. Journal of Geotechnical Engineering, 122(7): 534-543. doi:10.1061/(ASCE)07339410(1996)122:7(534).

Leroueil, S., Bouclin, G., Tavenas, F., Bergeron, L., and Rochelle, P. La. 1990. Permeability anisotropy of natural clays as a function of strain. Canadian Geotechnical Journal, 27(5): 568-579. doi:10.1139/t90-072.

Leroueil, S., Kabbaj, M., Tavenas, F., and Bouchard, R. 1985. Stress-strain-strain rate relation for the compressibility of sensitive natural clays. Géotechnique, 35(2): 159-180. doi:10.1680/geot.1985.35.2.159.

Leroueil, S., Morin, P., and Samson, L. 1983a. Preconsolidation pressure of Champlain clays . Part I . In-situ determination. Canadian Geotechnical Journal,: 782-802.

Leroueil, S., and Roy, A.N.D.M. 1978. construction pore pressure in clay conditions under embankments Part I : the Saint-Alban test fills. Canadian Geotechnical Journal,.

Leroueil, S., Samson, L., and Morin, P. 1983b. Preconsolidation pressure of Champlain clays. Part 2. Laboratory determination. Canadian Geotechnical Journal,: 803-816.

Leroueil, S., and Vaughan, P.R. 1990. The general and congruent effects of structure in natural soils and weak rocks. Géotechnique, 40(3): 467-488. doi:doi:10.1680/geot.1990.40.3.467.

Leussink, H. 1969. Storage yard foundations on soft cohesive soils. In In Proceedings of the 7th International Conference on Soil Mechanics and Foundation Engineering, Mexico City. pp. $149-155$.

Lewis, C.F.M., and Todd, B.J. 2019. The Early Lake Ontario barrier beach: evidence for sea level about 12.8-12.5 cal. ka BP beneath western Lake Ontario in eastern North America. Boreas, 48(1): 195-214. doi:10.1111/bor.12351.

Liu, J., Shi, C., Afroz, M., and Kirstein, A. 2017a. Numerical investigation of long-term settlement of Waba dam. Ontario Power Generation, Internal report. Unpublished.

Liu, M.D., and Carter, J.P. 2000a. on the Volumetric Deformation of Reconstituted Soils. International Journal for Numerical and Analytical Methods in Geomechanics, 133: 101-133.

Liu, M.D., and Carter, J.P. 2000b. Modelling the destructuring of soils during virgin compression. 
Géotechnique, (January). doi:10.1680/geot.2000.50.4.479.

Liu, M.D., and Carter, J.P. 2002. A structured Cam Clay model. Canadian Geotechnical Journal, 39(6): 1313-1332. doi:10.1139/t02-069.

Liu, M.D., and Carter, J.P. 2003. Volumetric Deformation of Natural Clays. International Journal of Geomechanics, 3(2): 236-252. doi:10.1061/(ASCE)1532-3641(2003)3:2(236).

Liu, W., Shi, M., Miao, L., Xu, L., and Zhang, D. 2013. Constitutive modeling of the destructuration and anisotropy of natural soft clay. Computers and Geotechnics, 51: 24-41. Elsevier Ltd. doi:10.1016/j.compgeo.2013.01.011.

Lo, K.Y., and Morin, J.P. 1972. Strength anisotropy and time effects of two sensitive clays. Canadian Geotechnical Journal, 9(3): 261-277. doi:10.1139/t72-030.

Lowe, J., Jonas, E., and Obrician, V. 1969. Controlled gradient consolidation test. Journal of the Soil Mechanics and Foundation Division, ASCE, 95(SM1): 77-97.

Mayne, P.W., and Kulhawy, F.H. 1990. Direct and indirect determinations of in situ K0 in clays. Transportation Research Record 1278,: 141-149.

McNutt, R.H., Frape, S.K., and Dollar, P. 1987. A strontium, oxygen and hydrogen isotopic composition of brines, Michigan and Appa- lachian Basins, Ontario and Michigan. Applied Geochemistry, 2: 495-505.

Mitchell, R.J. 1970. On the yielding and mechnical strength of Leda clays. Canadian Geotechnical Journal, 7(3): 297-312. doi:doi:10.1139/t70-036.

Monsif, M.Y., and Liu, J. 2019. Fundamental mechanism of cement in stabilizing Champlain Sea clay. In Proceedings of Geo-St. John's. pp. 1-8.

Mortara, G. 2015. A constitutive framework for the elastoplastic modelling of geomaterials. International Journal of Solids and Structures, 63: 139-152. Elsevier Ltd. doi:10.1016/j.ijsolstr.2015.02.047.

Mouratidis, A., and Magnan, J.P. 1982. Un modèle élastoplastique anisotrope avec écrouissage pour les argiles molles naturelles : Mélanie. Revue Française De Géotechnique, Numéro 25: $55-62$.

Nader, A. 2014. Engineering Characteristics of Sensitive Marine Clays - Examples of Clays in Eastern Canada. University of ottawa.

Nagaraj, B.T.S., Murthy, B.R.S., Vatsala, A., and Joshi, R.C. 1990. Analysis of Compressibity of Sensitive Clays. Journal of Geotechnical Engineering, 116(1): 105-118.

Nakai, T., and Matsuoka, H. 1986. A generalized elastoplastic constitutive model for clay in threedimensional stresses. Soils and Foundations, 26(3): 81-89. doi:10.1061/ASCE்109002412003'129:1'12.

Nova, R., and Wood, D.M. 1978. An experimental programme to define the yield function for sand. Soils and Foundations, 18(4): 77-86. doi:10.1061/ASCE்1090-02412003129:1'12.

Ontario Power Generation. 2008. Arnprior\&Waba Dam Performance Report.

Panayides, S., Rouainia, M., and Muir Wood, D. 2012. Influence of degradation of structure on the behaviour of a full-scale embankment. Canadian Geotechnical Journal, 49(3): 344-356. 
doi:10.1139/t11-104.

Pande, G.N., and Sharma, K.G. 1983. Multilaminate model of clays - a numerical evaluation of the influence of rotation of principal stress axes. International Journal for Numerical and Analytical Methods in Geomechanics, 7: 397-418.

Penner, E. 1965. A study of sensitivity in Leda Clay. Canadian Journal of Earth Sciences, 2(5): 425-441. doi:10.1139/e65-037.

Perzyna, P. 1963. The constitutive equations for work-hardening and rate sensitive plastic materials. In Proceedings of Vibration Problems. pp. 281-290.

Perzyna, P. 1966. Fundamental problems in viscoplasticity. Advances in Applied Mechanics, 9: 343-377.

Pietruszczak, S., and Pande, G.N. 2001. Description of soil anisotropy based on multi-laminate framework. International Journal for Numerical and Analytical Methods in Geomechanics, 25(2): 197-206. doi:10.1002/nag.125.

Plaxis. 2002. Plaxis Version 8 Material Models Manual.

Potts, D.M., Kovacevic, N., and Vaughan, P.R. 1997. Delayed collapse of cut slopes in stiff clay. Géotechnique, 47(5): 953-982.

Prévost, J.-H., and Höeg, K. 1975. Soil mechanics and plasticity analysis of strain softening. Géotechnique, 25(2): 279-297. doi:10.1680/geot.1975.25.2.279.

Quigley, R.M., Gwyn, Q.H.J., White, O.L., Rowe, R.K., Haynes, J.E., and Bohdanowicz, A. 1983. Leda clay from deep boreholes at Hawkesbury, Ontario. Part I: Geology and geotechnique. Canadian Geotechnical Journal, 20(2): 288-298. doi:10.1139/t83-032.

Reddy, B.K., Sahu, R.B., and Ghosh, S. 2015. Constant rate of strain consolidation of organic clay: in Kolkata region. International Journal of Geotechnical Engineering, 9(5): 471-482. Routledge. doi:10.1179/1939787914Y.0000000087.

Rezania, M., Nguyen, H., Zanganeh, H., and Taiebat, M. 2018. Numerical analysis of Ballina test embankment on a soft structured clay foundation. Computers and Geotechnics, 93: 61-74. Elsevier Ltd. doi:10.1016/j.compgeo.2017.05.013.

Roscoe, K.H., and Burland, J.B. 1968. On the generalized stress-strain behavior of "wet" clay. Engineering Plasticity,: 535-609. doi:10.1016/0022-4898(70)90160-6.

Rouainia, M., and D, M.W. 2000. A kinematic hardening model for natural clays with loss of structure. Géotechnique, 50(2): 153-164. doi:10.1680/geot.2000.50.2.153.

Schanz, T., Vermeer, A., and Bonnier, P. 1999. The hardening soil model: formulation and verification. Beyond 2000 Comput. Geotech. 10 years PLAXIS Int. Proc. Int. Symp. beyond 2000 Comput. Geotech. Amsterdam Netherlands 1820 March 1999,: 281.

Schweiger, H.F., Wiltafsky, C., Scharinger, F., and Galavi, V. 2009. A multilaminate framework for modelling induced and inherent anisotropy of soils. Géotechnique, 59(2): 87-101. doi:10.1680/geot.2008.3770.

Sekiguchi, H., and Ohta, H. 1977. Induced anisotropy and time dependency in clays. In Proc. 9th Int. Conf. Soil Mech. Found. Engng, Tokyo, Spec. pp. 229-238. 
Sheahan, T.C., and Watters, P.J. 1997. Experimental verification of CRS consolidation theory. Jounral of Geotechnical and Geoenvironmental Engineering,: 430-437.

Shi, C., and Salloum, T. 2018. Numerical study on excess pore pressure response of Champlain Sea Clay under 1-D constant rate of strain loading. In Proceedings of Geo-Edmonton 2018.

Silvestri, V. 1986. Controlled-Strain, Controlled-Gradient, and Standard Consolidation Testing of Sensitive Clays. Testing and Evaluation, ASTM STP 892,: 433-450.

Sivasithamparam, N., and Castro, J. 2015. An anisotropic elastoplastic model for soft clays based on logarithmic contractancy. International Journal for Numerical and Analytical Methods in Geomechanics,: 189-213. doi:10.1002/nag.

Sivasithamparam, N., Karstunen, M., and Bonnier, P. 2015. Modelling creep behaviour of anisotropic soft soils. Computers and Geotechnics, 69: 46-57. Elsevier Ltd. doi:10.1016/j.compgeo.2015.04.015.

Smith, R.E., and Wahls, H.E. 1969. Consolidation under constant rate of strain. Journal of the Soil Mechanics and Foundation Division, ASCE, 95(SM 1): 519-539.

Stermac, A.G., Devata, M., and Selby, K.G. 1968. Unusual movements of abutments supported on end-bearing piles. Canadian Geotechnical Journal, 5(2): 69-79.

Suebsuk, J., Horpibulsuk, S., and Liu, M.D. 2010. Modified Structured Cam Clay: A generalised critical state model for destructured, naturally structured and artificially structured clays. Computers and Geotechnics, 37(7-8): 956-968. Elsevier Ltd. doi:10.1016/j.compgeo.2010.08.002.

Tavenas, F., Chapeau, C., La Rochelle, P., and Roy, M. 1974. Immediate settlements of three test embankments on champlain clay. Canadian Geotechnical Journal, 11(109): 109-141. doi:10.1139/t74-008.

Tavenas, F., and Leroueil, S. 1977. The effect of stresses and time on yielding of clays. In Proceedings 9th International Conference Soil Mechanics and Foundation Engineering. pp. 319-326.

Tavenas, F., Mieussens, C., and Bourges, F. 1979. Lateral displacements in clay foundations under embankments. Canadian Geotechnical Journal, 16(3): 532-550. doi:10.1139/t79-059.

Torrance, J.K. 1974. A laboratory investigation of the effect of leaching on the compressibility and shear strength of Norwegian marine clays. Géotechnique, 24(2): 155-173. doi:10.1680/geot.1974.24.2.155.

Vatsala, A., Nova, R., and Murthy, B.R.S. 2001. Elastoplastic Model for Cemented Soils. International Journal of Geomechanics, ASCE, 127: 679-687.

Walker, L.K., and Raymond, G.P. 1969. Anisotropic consolidation of leda clay. Canadian Geotechnical Journal,: 271-286.

Wheeler, S.J. 1997. A rotational hardening elasto-plastic model for clays. 14th International Conference on Soil Mechanics and Foundation Engineering, 2: 6-9.

Wheeler, S.J., Anu, N., Karstunen, M., and Matti. 2003. An anisotropic elastic-viscoplastic model for soft clays. Canadian Geotechnical Journal, 40: 403-418. doi:10.1016/j.ijsolstr.2009.11.004. 
Wiltafsky, C., Messerklinger, S., and Schweiger, H.F. 2002. An advanced multilaminate model for clay. In Proceedings of the 8th International Symposium on Numerical Models in Geomechanics-NUMOG VIII, Rome, Italy. pp. 67-73.

Wissa, A.E.Z. 1971. Consolidation at constant rate of strain. Jounral of the Soil Mechanics and Foundations Division - Proceeding of the American Society of Civil Engineers,: 1393-1413.

Wood, D.M. 1994. Soil Behaviour and Critical State Soil Mechanics. Cambridge University Press.

Wood, D.M., and Belkheir, K. 1994. Strain softening and state parameter for sand modelling. Géotechnique, 44(2): 335-339. doi:10.1007/BF00857417.

Yang, C., Liu, X., Liu, X., Yang, C., and Carter, J.P. 2015. Constitutive modelling of Otaniemi soft clay in both natural and reconstituted states. Computers and Geotechnics, 70: 83-95. Elsevier Ltd. doi:10.1016/j.compgeo.2015.07.018.

Yao, Y.P., Sun, D.A., and Matsuoka, H. 2008. A unified constitutive model for both clay and sand with hardening parameter independent on stress path. Computers and Geotechnics, 35(2): 210-222. doi:10.1016/j.compgeo.2007.04.003.

Yildiz, A., Karstunen, M., and Krenn, H. 2009. Effect of anisotropy and destructuration on behavior of Haarajoki test embankment. International Journal of Geomechanics, 9(June): 153-168. doi:10.1016/j.buildenv.2006.10.027.

Yildiz, A., and Uysal, F. 2016. Modelling of anisotropy and consolidation effect on behaviour of sunshine embankment: Australia. Australian Library Journal, 14: 83-95. Springer International Publishing. doi:10.1007/s40999-016-0018-1.

Yin, J.-H., and Graham, J. 1990. Viscous-elastic-plastic modelling of one-dimensional timedependent behaviour of clays. Canadian Geotechnical Journal, 27(2): 262-265. doi:10.1139/t90-032.

Yin, J.-H., and Graham, J. 1999. Elastic viscoplastic modelling of the time-dependent stress-strain behaviour of soils. Canadian Geotechnical Journal, 36(4): 736-745. doi:10.1139/t99-042.

Yin, J.-H., and Zhu, J.-G. 1999. Measured and predicted time-dependent stress-strain behaviour of Hong Kong marine deposits. Canadian Geotechnical Journal, 36(4): 760-766. doi:10.1139/t99-043.

Yin, J.-H., Zhu, J.-G., and Graham, J. 2002. A new elastic viscoplastic model for time-dependent behaviour of normally and overconsolidated clays: theory and verification. Canadian Geotechnical Journal, 39: 157-173. doi:10.1139/t01-074.

Yin, J., and Graham, J. 1994. Equivalent times and one-dimensional elastic viscoplastic modelling of time-dependent stress-strain behaviour of clays. Canadian Geotechnical Journal,: 42-52.

Yin, J.H. 2003. Calculation of settlements of foundation soils considering creep. In Engineering in Coastal Area - Proceedings of the Nakase Memorial Symposium, Yokosuka, Japan. pp. 205-212. doi:doi:10.1201/9781439833919.ch23.

Yin, Z., Karstunen, M., Chang, C.S., Asce, M., Koskinen, M., and Lojander, M. 2011. Modeling Time-Dependent Behavior of Soft Sensitive Clay. Journal of Geotechnical and Geoenvironmental Engineering, 137(November): 1103-1113. doi:10.1061/(ASCE)GT.1943-5606.0000527. 
Yin, Z.Y., Chang, C.S., Karstunen, M., and Hicher, P.Y. 2010. An anisotropic elastic-viscoplastic model for soft clays. International Journal of Solids and Structures, 47(5): 665-677. Elsevier Ltd. doi:10.1016/j.ijsolstr.2009.11.004.

Yin, Z.Y., and Karstunen, M. 2008. Influence of Anisotropy, Destructuration and Viscosity on the Behavior of an Embankment on Soft Clay. 12th International Conference of IACMAC, (March): 4728-4735.

Zabala, F., and Alonso, E.E. 2011. Progressive failure of Aznalcóllar dam using the material point method. Géotechnique, 61(9): 795-808. doi:10.1680/geot.9.P.134.

Zentar, R., Karstunen, M., Wiltafsky, C., Schweiger, H.F., and Koskinen, M. 2002. Comparison of two approaches for modelling anisotropy of soft clays. In Proceedings of the 8th International Symposium on Numerical Models in Geomechanics-NUMOG VIII, Rome, Italy. pp. 115-121.

Zienkiewicz, O.C., and Pande, G.N. 1977. Time dependent multi-laminate model of rocks: a numerical study of deformation and failure of rock masses. International Journal for Numerical and Analytical Methods in Geomechanics, 1(3): 219-247. 
